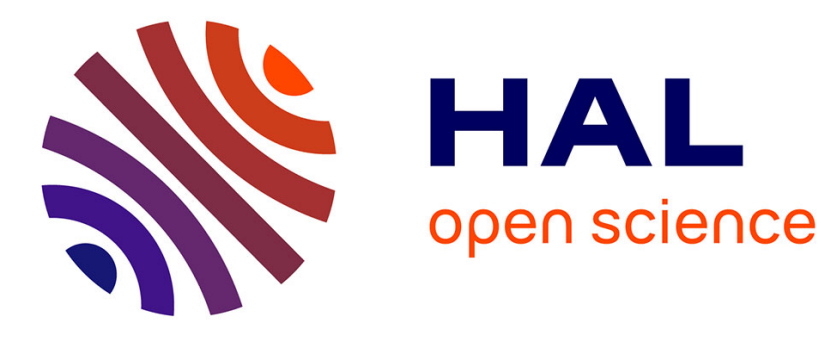

\title{
Les ingénieurs maghrébins dans les systèmes de formation
}

Eric Gobe, Azzedine Ali Ben Ali, Mohammed Benguerna, Habib Belaïd, Saïd Ben Sedrine, Mohamed El Faïz, André Grelon, Mustapha Haddab, Anousheh Karvar, Hocine Khelfaoui, et al.

\section{To cite this version:}

Eric Gobe, Azzedine Ali Ben Ali, Mohammed Benguerna, Habib Belaïd, Saïd Ben Sedrine, et al.. Les ingénieurs maghrébins dans les systèmes de formation: Systèmes de formationFilières coloniales et pratiques professionnellesProfessionnalités contemporaines. Institut de recherche sur le Maghreb contemporain de Tunis. Pas d'éditeur commercial, pp.225, 2001. halshs-00120303

\section{HAL Id: halshs-00120303 \\ https://shs.hal.science/halshs-00120303}

Submitted on 22 Dec 2006

HAL is a multi-disciplinary open access archive for the deposit and dissemination of scientific research documents, whether they are published or not. The documents may come from teaching and research institutions in France or abroad, or from public or private research centers.
L'archive ouverte pluridisciplinaire HAL, est destinée au dépôt et à la diffusion de documents scientifiques de niveau recherche, publiés ou non, émanant des établissements d'enseignement et de recherche français ou étrangers, des laboratoires publics ou privés. 


\title{
LES INGÉNIEURS MAGHRÉBINS DANS LES SYSTĖMES DE FORMATION
}

\author{
Systèmes de formation \\ Filières coloniales et pratiques professionnelles \\ Professionnalités contemporaines
}

Sous la direction d'Eric GOBE

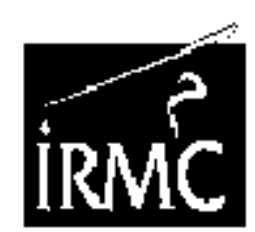

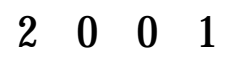


Publication non périodique diffusée gratuitement sur demande

IRMC

20, rue Mohamed Ali Tahar

Mutuelleville - 1002 TUNIS

Téléphone : (01) 796722

Fax : (01) 797376

E-mail : mail@irmcmaghreb.org

http://www.irmcmaghreb.org 


\title{
Institut de Recherche sur le Maghreb Contemporain
}

\section{LES IN GÉN IEURS MAGHRÉBINS DANS LES SYSTĖMES DE FORMATION}

\author{
Systèmes de formation \\ Filières coloniales et pratiques professionnelles \\ Professionnalités contemporaines
}

Actes de la réunion intermédiaire du programme Ingénieurs et société au M aghreb placée sous la responsablité scientifique d'Eric GOBE, organisée par l'IR M C à Rabat, les 2 et 3 février 2001 Introduction d'A ndré G RELON, directeur d'études à l'EHESS

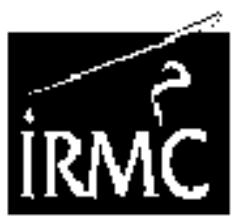

20001 



\section{So m m a i re}

Eric G O BE , Avant-propos. 9

André GRELON, Introduction : Ingénieurs et sociétés dans le Maghreb contemporain. L'itinéraire d'un programme de recherche.

\section{ChAPITRE 1}

\section{Systèmes de formation}

\section{Le modèle de formation en question}

Kamel MELLAKH , La formation des ingénieurs par le système d'enseignement supérieur au Maroc. .29

Saïd Ben SEDRINE et Eric GOBE, Les ingénieurs tunisiens dans le système éducatif : quel modèle de formation pour les cadres techniques?.

Le modèle des élites techniques en question

A nousheh KARVAR, La formation des élèves algériens, tunisiens et marocains à I'Ecole polytechnique française (1921-2000) : des acteurs de l'histoire aux « élites de peu ». .79

Mohamed BENGUERNA, L'Ecole polytechnique d'Alger : la formation inachevée d'une élite technique .101

G razia SCARFO-GHELLAB, Les écoles d'ingénieurs : lieux de production et de reproduction d'une fraction des élites marocaines ? 


\section{La relation formation-emploi}

Mustapha HADDAB, Evolutions dans la formation et le statut social des ingénieurs en Algérie.

Azzeddine ALI BENALI, Les ingénieurs pétroliers de l'Institut algérien du pétrole : formation et parcours professionnels

\section{ChAPITRE 2}

Filières coloniales et pratiques professionnelles.

H abib BELAÏD, Figures d'ingénieurs pendant le protectorat français en Tunisie: l'exemple de la Poste et des Travaux publics. 149

Hélène VACHER, Du métier à la profession : I'émergence de l'ingénieur géomètre et l'exercice colonial au début du XXe siècle. .173

\section{Chapitre 3 \\ Professionnalités contemporaines}

Hocine KHELFAOUI, Evolution du profil de l'ingénieur algérien : du « technicien » au «développeur »? .197

Mohammed EL FAÏZ, La Grande Hydraulique dans le Haouz de Marrakech : fascination technologique et émergence du pouvoir des ingénieurs. .213 


\section{Liste des auteurs}

ALI BENALI Azzedine, étudiant en DEA à l'Ecole des hautes études en sciences sociales (EHESS), Paris.

BENGUERNA Mohammed, chercheur au Centre de recherches en économie appliquée au développement (CREAD), Alger.

BELAIID Habib, chercheur à l'Institut supérieur d'histoire du mouvement national (ISHMN), Université de la Manouba, Tunis.

BEN SEDRINE Saïd, enseignant à I'Institut national du travail et des études sociales (INTES), Tunis.

EL FAÏZ Mohamed, responsable de I'UFR « Analyse économique et développement », Faculté de sciences juridiques, économiques et sociales, Université Cadi Ayyad, Marrakech.

GOBE Eric, chercheur à I'Institut de recherche sur le Maghreb contemporain (IRMC), Tunis.

GRELON André, directeur d'études à l'École des hautes études en sciences sociales (EHESS), LASMAS-CNRS, Paris.

HADDAB Mustapha, professeur à I'Institut de psychologie et de sciences de l'éducation, Université d'Alger.

KARVAR A nousheh, chargée de mission d'étude et de recherche à I'UCC - CFDT, Paris.

KHELFAOUI Hocine, maître de recherche associé au Centre de recherches en économie appliquée au développement (CREAD), Alger.

MELLAKH Kamel, maître de conférence en sociologie à I'Université d'Agadir.

SCA RFO-GHELLAB Grazia, professeur à l'Ecole Hassania des travaux publics de Casablanca.

VACHER Hélène, chargée de recherche à I'Université d'Aalborg, Danemark, chercheur à URBAMA Tours, et chercheur associé au CDHT CNAM/ EHESS, Paris. 



\section{A vant-propos}

Cet ouvrage constitue la première publication collective du programme de l'IRMC Ingénieurs et société au M aghreb. II rassemble la plupart des contributions de la rencontre thématique de Rabat (2-3 février 2001) qui portait plus particulièrement sur « Les ingénieurs maghrébins dans les systèmes de formation ».

Les articles publiés dans ce recueil sont des textes intermédiaires qui font état des réflexions des membres du programme. Les participants à la rencontre de Rabat étaient al ors à divers stades d'avancement de leurs travaux. Certains chercheurs ont présenté des contributions énonçant les hypothèses et les perspectives de leur recherche, tandis que d'autres ont évoqué les premiers résultats d'enquêtes déjà achevées.

Pourquoi avoir choisi de se pencher de manière spécifique sur la question de la formation des ingénieurs marocains, algériens et tunisiens? Si la question del'emploi des diplômés au Maghreb a étélargement abordée, tant par le discours des politiques que des chercheurs $^{1}$, en revanche la communauté scientifique a négligé l'étude des formations d'ingénieurs. Or, ces dernières apparaissent stratégiques en terme de développement dans la mesure où elles sont censées fournir la main-d'œuvre qualifiée, les cadres et les innovateurs du secteur productif.

Au Maghreb, l'effort de formation a été d'autant plus massif que la présence de Maghrébins dans les corps techniques au moment de l'indépendance était insignifiante. Cependant, au-delà des discours sur la nécessité de former des ingénieurs et des techniciens, la question de la formation des compétences se pose moins aujourd'hui en termes de pénurie de cadres techniques, qu'en termes de massification de certaines formations d'ingénieurs. Il convient désormais de s'attarder sur les implications scolaires, professionnelles et sociales des arbitrages imposés par le nouveau contexte de la transition libérale ou d'austérité budgétaire qui touche les États maghrébins. L'horizon professionnel des diplômés des écoles d'ingénieurs est plus rétréci que dans les trois 
premières décennies des indépendances. L'administration et les entreprises publiques restent le premier employeur des ingénieurs au Maghreb ; mais elles recrutent désormais au compte-gouttes. Certaines catégories d'ingénieurs se trouvent déqualifiées, voire déclassées et, parfois, au chômage. Si cette situation est moins dramatique pour les jeunes diplômés ingénieurs que pour d'autres catégories de diplômés, il n'en demeure pas moins qu'ils connaissent un processus d'insertion professionnelle plus lent que dans les années 1980. Par ailleurs, la perspective de carrières peu attractives dans leurs propres pays incitent certains ingénieurs formés à l'étranger, dans des écoles d'élites, à ne pas «rentrer au pays ».

N'y a-t-il pas danger, dans un tel contexte, de voir les gestionnaires du système éducatif réduire fortement les effectifs des filières technologiques de haut niveau, sans prendre en compte le manque à gagner qu'une telle politique produirait du point de vue des savoirs scientifiques sur les sociétés et les systèmes de gouvernement ?

Par ailleurs, I'histoire de la figure de l'ingénieur au Maghreb a été abordée lors de cette rencontre. Des historiens ont posé des jalons permettant de mieux comprendre les pratiques professionnelles et le discours des ingénieurs de la période coloniale. Last but not least, l'étude des professionnal ités d'ingénieurs à l'époque contemporaine nous a permis d'appréhender les fonctions exercées et le rôle joué par les ingénieurs dans l'organisation, en interaction avec leur environnement extérieur.

Eric G OBE

1 Voir à ce sujet Vincent GEISSER (dir.), Diplômés maghrébins d'ici et d'ailleurs. Trajectoires et itinéraires migratoires, Paris, Ed. du CNRS, 2000. L'ouvrage présente les résultats du programme de recherche del'IRMC «Flux et gestion des compétences intellectuelles dans les échanges euro-maghrébins », conduit par Vincent Geisser de 1995 à 1998. 


\title{
Introduction
}

\section{Ingénieurs et sociétés dans le M aghreb contemporain L'itinéraire d'un programme de recherche}

\begin{abstract}
André GRELON
A l'issue de deux rencontres du programme d'études dirigé par Eric Gobe sous l'égide de I'Institut de recherche sur le Maghreb contemporain sur les ingénieurs au Maghreb, leurs rôles professionnels, leur place dans la vie politique, économique et sociale dans chacun des pays considérés, il importe de commencer à tirer quelques traits d'un bilan du travail, mais aussi de réfléchir à une suite possible des travaux. L'ampleur des questions qui ont été soulevées invite à considérer que, malgré la qualité des études menées, tout n'a pas pu être abordé. Ou plus exactement, à cause même de la qualité de ces études, des problèmes cachés ou discrets ont émergé, soulevant de nouvelles hypothèses et incitant à entreprendre de nouvelles investigations. Dès la fin des années 1950 au Maroc et en Tunisie, dès 1962 en A lgérie, la question des ingénieurs, liée au rôle que les dirigeants politiques entendaient leur faire jouer, a été considérée comme fondamentale pour l'avenir de ces pays. Aujourd'hui, les difficultés économiques soulèvent des problèmes d'une autre nature, en particulier ceux de l'emploi des ingénieurs formés en grand nombre et spécialement des jeunes diplômés tout juste sortis des établissements de formation. Toutefois on ne peut pas fonder une analyse de la situation actuellesi l'on n'a pas entrepris de dégager l'ensemble du processus d'évolution de ce groupe professionnel depuis son origine. Quelques pistes de réflexion élaborées à partir des interventions des uns et des autres et des communications de la réunion de Rabat sont présentées ci-après.
\end{abstract}




\section{LE MAGHREB DES INGÉNIEURS DANS L'HISTOIRE : RUPTURE COLONIALE ET INDÉPENDANCE}

Dès le début du programme de recherche, nous avons été confrontés à une double perspective apparemment contradictoire, mais dont il nous faut bien rendre compte simultanément.

D'une part, une rupture entre la période coloniale et l'ère de l'indépendance. Les travaux publiés sous la direction d'Elisabeth Longuenesse, et notamment ceux de Lilia Ben Salem¹, l'avaient déjà établi ; les recherches menées depuis et présentées par les divers intervenants de chacun des trois pays le confirment : il n'existe qu'une continuité ténue entre les ingénieurs de la période coloniale et ceux apparus après l'indépendance. Trois raisons sont avancées par les chercheurs :

$1^{\circ}$ La politique coloniale n'a pas favorisé, loin de là, l'émergence d'une élite technique locale. Les constats dressés par Habib Belaïd pour la Tunisie, par Kamel Mellakh au Maroc, et par Mohamed Benguerna dans son enquête sur les premiers ingénieurs al gériens le démontrent à l'envi : au moment des indépendances, il n'y avait qu'une poignée d'ingénieurs nationaux pour prendre la relève. A cet égard, les statistiques établies par Anousheh Karvar concernant les polytechniciens en provenance du Maghreb à partir de 1921 sont sans appel.

$2^{\circ}$ Le développement économique sous le régime colonial n'a pas privilégié I'industrialisation du Maghreb. Aussi les établissements de formation qui accompagnent la constitution de pôles industriels pour doter ceux-ci de techniciens spécialisés ne pouvaient-ils être créés. En France métropolitaine, avant la Seconde Guerre mondiale, les écoles d'ingénieurs étaient de toute manière essentiellement implantées dans les régions à caractère traditionnellement industriel : Paris, Lille, Lyon, Grenoble. Les régions rurales en étaient dépourvues. Ce n'est qu'à partir des années soixante que des établissements d'enseignement technique supérieur ont été fondés sur l'ensemble de l'H exagone, avec une volonté d'aménagement et de valorisation du territoire (Tarbes, Brest, Metz...) et notamment dans le cadre des nouvelles universités (Orléans, Valenciennes, La Rochelle, etc.). Dans la conception antérieure, il était donc logique de ne pas implanter des écoles d'ingénieurs à vocation industrielle dans les colonies. D'autant que la miseen place d'une école est un investissement lourd (bâtiments, matériel, personnel) qui doit rencontrer son public (ce qui suppose un bassin de recrutement régional déjà constitué) et supposer des débouchés : I'expérience de l'Ecole de géomètres et de dessinateurs implantée à Rabat en 1920 et qui ferme deux ans plus tard illustre les difficultés d'une telle entreprise. Hélène 
Vacher montre qu'en définitive, il s'est avéré plus efficace de distribuer des bourses pour envoyer les lauréats se former à l'Ecole des travaux publics à Paris. En revanche, la création d'un établissement de formation agricole à Tunis, en 1898, répondait à des besoins locaux, et spécialement ceux des colons français. Elles'inscrivait en outre dans un contexte de rationalisation de l'agriculture qui inspirait la III République, marqué notamment par l'ouverture de plusieurs établissements spécialisés métropolitains : Institut agricole et Institut colonial de $\mathrm{N}$ ancy, Ecole des industries agricoles de Douai, Ecole du génie rural, etc.

$3^{\circ}$ Dans une telle situation, l'idéal de la notabilité, tant dans les couches bourgeoises coloniales que dans les milieux aisés maghrébins, ne se situait pas dans les métiers techniques, mais bien plutôt dans les classiques professions libérales : médecin, avocat. Comme l'explique Mustapha Haddab, les Algériens - y compris dans la communauté européenne - n'étaient guère nombreux à avoir acquis une connaissance concrète du personnage de l'ingénieur. Les ingénieurs implantés dans les colonies étaient peu nombreux et très général ement issus de la métropole, soit qu'ils fussent en position de fonctionnaire dans I'administration d'Etat (Ponts et chaussées, Génie rural, PTT...), soit en tant que cadres d'entreprises privées (sociétés de travaux publics, par exemple). A ce titre, en s'appuyant sur les annuaires des associations d'anciens élèves, une étude pourrait être menée pour examiner les profils professionnels de ceux qui partaient « aux colonies ».

Par conséquent, même si l'on peut noter des « héritages » - comme par exemple une filiation entre l'ancien Institut technique d'A Iger et la nouvelle Ecole polytechnique d'Alger tel que le montre Mohamed Benguerna, des passations de pouvoir d'une administration française à une administration tunisienne ainsi que le décrit $\mathrm{Habib}$ Belaïd - le moment historique de l'indépendance ou du retour à la souveraineté pleine et entière crée bien une situation totalement neuve avec la définition d'objectifs nationaux urgents par les responsables politiques : d'une part, remplacer les experts techniques disparus, déjà dans l'administration afin de faire tourner la machine de l'Etat, mais aussi dans les entreprises pour assurer une transition économique sans trop de heurts ; d'autre part, ancrer une ambition de mise en valeur des richesses du pays et positionner celui-ci à bon rang dans l'échelle internationale du développement, par une politique volontariste de formation des cadres techniques, censés être le moteur du dynamisme économique. Comme l'écrivent Saïd Ben Sedrine et Eric Gobe, «l'ingénieur était (...) perçu par les autorités des Etats issus de la décolonisation comme l'agent du développement industriel et le détenteur du secret de la modernité». 
II faudra examiner si les débats qui ont eu lieu en France dès le début des années 1950 sur la question du manque d'ingénieurs et de ses effets sur l'économie ont rencontré un écho chez les responsables politiques maghrébins. Le problème qui était posé dans les milieux économiques était celui du retard français par rapport aux autres pays del'OCDE (à l'époque OECE) et des freins au développement de ce qu'on appelait dans le langage du moment «les industries de pointe », la chimie et notamment la chimie fine, les industries pétrolières, la construction mécanique, l'aéronautique... Dans ces secteurs, on disait souffrir du manque de cadres techniques formés, ce qui handicapait la croissance des entreprises. La réponse volontariste fut donnée par l'Etat avec la création de nouveaux types d'établissements de formation - les Instituts nationaux de sciences appliquées (INSA) et les Ecoles nationales d'ingénieurs (ENI) - nonobstant la résistance des milieux d'ingénieurs qui craignaient une augmentation incontrôlée du nombre de diplômés. De tels phénomènes ont-ils joué un rôle dans l'établissement de la politique d'enseignement supérieur des pays maghrébins?

Par ailleurs, il n'en reste pas moins indispensable de prendre en compte la dimension historique plus ancienne : car même mandatés pour des objectifs nouveaux, les nouveaux ingénieurs maghrébins ne débarquaient pas sur un terrain vierge. Les territoires coloniaux avaient fait l'objet de nombreuses interventions techniques et avaient été déjà largement modelés par le travail de l'ingénieur. Ainsi, dans son article sur les ingénieurs et la politique hydro-agricole au $\mathrm{Maroc}^{2}$, Jean-Jacques Pérennès a montrél'ampleur des débats chez les ingénieurs français des travaux publics à propos de la construction de barrages et de la mise en valeur des terres arides dans les pays du Maghreb au cours du XIX siècle. L'option prise finalement en faveur de la grande hydraulique au début du $X X^{e}$ siècle favorisait objectivement les grandes entreprises françaises de BTP. Mais au-delà du caractère de rentabilité capitalistique de cette orientation, il faut aussi prendre en compte le mode de rationalité des ingénieurs, promoteurs de ces projets. Pour ceux-ci, en mettant la technique la plus neuve aux postes de commande, on aboutissait naturellement à la solution la plus adéquate dans chacun des domaines considérés, celle qui menait à un avenir meilleur, face aux porteurs de traditions rétrogrades, aux tenants de la routine, aux milieux fixés sur un présent sans devenir. Ce mode de pensée n'a pas disparu avec l'indépendance, bien au contraire. A nimés par un élan patriotique, convaincus qu'ils étaient d'être investis d'une mission historique, les ingénieurs de l'indépendance ont mis au service de cet idéal un raisonnement, une manière d'être fondée sur une croyance en l'objectivité des faits, sur 
une analyse des données en vertu de considérations scientifico-techniques, se coulant ainsi dans les orientations laissées par leurs devanciers. Mohamed El Faïz est revenu sur cette dimension historique dont l'examen est indispensable pour mettre en perspective les actuelles réalisations des ingénieurs hydrauliciens marocains, que leurs travaux soient approuvés ou contestés. En sens inverse, examinant le cas des ingénieurs topographes, Hélène Vacher s'inscrit en faux contre l'idée reçue selon laquelle les agents techniques employés dans les colonies n'auraient eu qu'une pure fonction instrumentale de diffusion des techniques. Elle met en évidence comment, pour les topographes, le fait de pratiquer leur métier dans les aires coloniales a eu une incidence non négl igeable sur l'ensemble de la profession elle-même. Au-delà, elle éclaire le rôle qu'ont pu jouer les colonies dans les stratégies de modernisation administrative et technique de la France métropolitaine. Ce développement de disciplines dans des perspectives originales, ces mises en œuvre de pratiques spécifiques contribuent à un enrichissement universel de l'art de l'ingénieur. Ainsi, on peut rattacher des études liées à des aires culturelles particulières à des considérations générales quant à la profession d'ingénieur prise dans sa globalité : chaque avancée technique proposée et réalisée par un ingénieur particulier ou un groupe d'ingénieurs désignés participe du renforcement de la figure omnisciente de l'Ingénieur.

\section{LA PROBLÉmATIQUe De LA FORMATION DES INGÉNIEURS : ENTRE « ÉLITISME 》ET « MASSIFICATION 》}

La question des diplômes et du nombre de diplômés, de l'évolution de la quantité d'ingénieurs estampillés est récurrente. Les statistiques sont en principe disponibles ou peuvent être reconstituées et des tableaux peuvent êtredressés. Tant qu'on est dansl'ordre du comptage, les choses sont relativement simples. De ce premier point devue, il serait déjà intéressant de tirer des informations comparatives entre les trois pays concernés:

- Evolution du nombre de diplômés depuis l'indépendance (ou le retour à la souveraineté). Ces chiffres dans l'absolu donnent une première indication. Mais ils doivent être immédiatement rapportés à l'évolution générale de la population universitaire et à l'évolution générale de celle-ci par rapport aux classes d'âge concernées. Ce sont évidemment ces ratios qui sont instructifs et permettent de mesurer l'ampleur de l'effort éducationnel des trois Etats - spécialement dans le domaine de la formation des experts techniques. A ce titre, il serait utile de disposer de courbes indiquant l'évolution du nombre de diplômés ingénieurs par rapport aux cadres de gestion et de commerce, que ceux-ci soient formés dans le cadre général universitaire ou dans des structures ad hoc comme les écoles de gestion. 
- Sur cette base générale, on peut affiner les renseignements : ainsi on peut relever l'évolution des différentes spécial ités telles qu'elles sont désignées dans chacun des pays. L'exercice est un peu plus périlleux : d'une part, parce que la définition de telle ou telle spécialité et les regroupements disciplinaires et sous-disciplinaires qu'elle implique peut être propreà chaque pays et la comparaison devient de ce fait plus délicate; d'autre part, parce qu'on sait bien que les termes mêmes de spécialités enferment trop les ingénieurs dans des cases dont ils sortiront peu ou prou dans l'exercice de leur profession. D'autres données peuvent être extraites qui sont porteuses de significations politiques, sociales et culturelles : la distinction entre les diplômés issus de cursus universitaires et ceux issus d'instituts techniques, la proportion des ingénieurs formés sous la tutelle du ministère chargé de l'Education en regard de ceux sortis d'établissements dépendant de ministères techniques (par exemple des ingénieurs d'instituts pétroliers, tel l'Institut algérien du pétrole examiné par Azzedine Ali Benali ou d'instituts agronomiques), l'évolution de la proportion de femmes ingénieurs dans les différents types d'établissement et les différentes spécialités. Enfin on ne peut négliger les données classiques sur l'origine socioprofessionnelle (ce qui soulève des questions relevant de la sociologie générale sur la nature des classements sociaux, la réalité des catégories sociales, les modes d'usage sociologique, l'influence de modèles étrangers, notamment de l'INSEE français...) ainsi que les questions sur l'origine géographique qui peuvent être liées éventuellement à des problèmes plus délicats de représentation de minorités ethniques.

Ces données statistiques sont indispensables comme base de travail pour avoir une première vision de la population concernée, pour comprendre les tendances de l'évolution générale de la formation technique supérieure et la politique d'investissement dans ce domaine de chacun des pays, et notamment pour mesurer les efforts massifs accomplis depuis les indépendances dans une volonté de « rattrapage». Elles permettent aussi de situer ces Etats dans une comparaison internationale plus large, par rapport à des modèles d'éducation, notamment avec la France, mais aussi avec d'autres pays d'Europe.

De ce point de vue, le travail exemplaire mené par Saïd Ben Sedrine et Eric Gobe sur les ingénieurs tunisiens devrait pouvoir servir de base pour pouvoir établir des comparaisons avec les deux autres pays. A terme, il s'agirait de mettre en place un outil permanent, un observatoire de la profession d'ingénieur dans les pays du Maghreb en affinant progressivement les données statistiques. 
On ne peut pour autant limiter l'étude de la formation à un rassemblement ordonné d'indications quantitatives sur les diplômés. Il importe d'examiner le processus même de la formation, son organisation, la définition des cursus et leur évolution dans le temps. Le travail de recherche, s'appuyant souvent sur des études antérieures particulières, a permis d'avancer largement dans cette voie pour chacun des pays. II importerait désormais sur la base de ces acquis d'entreprendre une comparaison raisonnée pour déterminer, hors de tout syncrétisme, ce qui est commun aux trois pays concernés, et notamment dans ce qui relèverait du rapport général de l'aire culturelle avec le fait colonial passé dans le domaine de la formation technique supérieure comme dans les rapports actuels avec l'ancienne puissance coloniale, parce qu'à l'évidence, il s'agit là de points majeurs ; mais aussi ce qui rapprocherait sur tel ou tel point deux des pays sur les trois, et ce qui est caractéristique de chaque Etat, de son développement particulier, en référence à ses spécificités économiques et géographiques, à son discours développementaliste, à son histoire politique. Sans prétendre ici lister l'ensemble des questions susceptibles d'être examinées, mentionnons-en quelques-unes comme base de discussion.

* L'externalisation d'une partie de la formation des ingénieurs : dans chaque pays et selon des modalités et des rythmes différents, une partie des ingénieurs a été envoyée se former dans des pays étrangers et spécialement dans l'ancien pays colonisateur en raison de la formation scolaire secondaire préparant bien au passage dans le système spécifique de l'enseignement technologique supérieur français (classes préparatoires + concours + école d'ingénieurs), en raison de la langue, mais aussi de la présence de groupes d'ingénieurs formés en France avant les indépendances et dont l'influence était sans commune mesure avec la taille de leur groupe. Ce phénomène qui aurait pu n'être que transitoire, compte tenu de la mise en place progressive de structures locales de formation des ingénieurs, est devenu un fait permanent, même si les flux d'étudiants se sont progressivement amoindris en raison de la concurrence légitime des écoles autochtones, mais aussi d'une part, de la faible rentabilité de l'opération due à une perte en ligne d'un pourcentage important de jeunes ingénieurs qui décident de ne pas revenir au pays et d'autre part, de la politique restrictive d'accueil d'étudiants étrangers et notamment maghrébins en France durant la dernière décennie - politique toujours susceptible d'être modifiée quand la crainte d'une perte du rayonnement culturel français devient trop intense chez les dirigeants politiques français. On notera que chaque pays a créé rapidement au moins un établissement « de prestige » - l'Ecole nationale d'ingénieurs de Tunis, l'Ecole polytechnique d'Alger, l'Ecole Mohammedia d'ingénieurs 
de Rabat - dans le but affiché de former ses élites techniques sur place, avec des réussites diverses commele démontrent Kamel Mellakh et Grazia Scarfo Ghellab étudiant lecas du Maroc. II est clair aujourd'hui que l'excellence scolaire des candidats retenus dans les écoles françaises se conjugue avec une sélection sociale drastique et on peut s'interroger sur les stratégies d'expatriation mises en place par les couches privilégiées pour leurs générations montantes.

* La mise en place du système de formation des ingénieurs reprend, malgré quelques adaptations locales, le modèle français. Aucun des auteurs du programme de recherche ne fait mention d'une mise en question ou à tout le moins d'un débat concernant l'organisation de l'enseignement supérieur de l'ancienne puissance coloniale. L'empreinte du système mis en place sous la III République est si prégnante que son application aux réalités de jeunes pays va comme allant de soi. Déjà l'organisation de l'enseignement supérieur apparaît immédiatement comme un monopole d'Etat, à plus forte raison encore à propos de la formation des ingénieurs à qui sont assignés une tâche spéciale dans la mise en valeur du pays. Cette situation ressortit, selon Eric Gobe et Saïd Ben Sedrine, à l'organisation étatique du travail technique. Mais deux caractéristiques sont reprises directement du système français, même lorsque les Etats sont indépendants depuis quelques années et que leurs responsables ont pu prendre du recul :

$1^{\circ}$ Une distinction s'établit entre formations générales d'ingénieurs relevant de I'Education nationale et formations techniques supérieures sous tutelle des ministères techniques : en Algérie, ces instituts ont fait l'objet d'une étude systématique par Hocine Khelfaoui. Il s'ensuit une classification complexe entre les établissements en fonction à la fois du prestige scolaire - sur la base de critères de sélection académique -, de la rentabilité professionnelle du diplôme, du rang dans la hiérarchie du pouvoir des ministères de tutelle, etc. qui finit par égaler en complexité le classement français. Même si les sociologues ont pris I'habitude en France de ranger les établissements de formation en deux catégories commodes, en terme de «grande » et de «petite porte », pour reprendre la célébrissime distinction de Pierre Bourdieu ${ }^{3}$, I'organisation hiérarchique des écoles d'ingénieurs est aussi raffinée et incompréhensible pour le commun des mortels que ne l'était le classement nobiliaire à la cour des rois de France sous l'ancien régime.

$2^{\circ}$ Le deuxième point qui découle du premier est la distinction qui s'établit entre les écoles d'ingénieurs dans leur ensemble (qu'elles soient ou non intégrées dans le système universitaire) et les formations universitaires classiques. Analysant le cas tunisien, Saïd Ben Sedrineet Eric Gobevont jusqu'à parler de relégation en cequi concerne 
les universités, les instituts de gestion, les facultés de médecine et certaines écoles d'ingénieurs « écrémant » systématiquement chaque nouvelle génération parvenant à l'enseignement supérieur, et ne laissant plus aux facultés des sciences queles éléments les plus faibles. Sans doute n'existe-t-il pas dans le monde de modèle magique d'enseignement supérieur qui permettrait une parfaite égalité des chances dans les études et l'accès de tous à l'emploi de son choix. Chaque système national est toujours lié à I'histoire globale du pays : ainsi, il n'y a pas d'autre explication à la constitution, au fil du temps, des hiérarchies d'écoles d'ingénieurs autonomes en France, comme à l'émergence des universités techniques en Allemagne. Les universités françaises sont nées à la fin du XIX ${ }^{e}$ siècle avec le handicap congénital d'apparaitre après la création de toute une série d'établissements parfaitement reconnus qui n'avaient eu ni référence universitaire pour élaborer leur cursus et constituer leur mode d'organisation, ni comme horizon de diffuser le savoir le plus large au plus grand nombre, mais au contraire d'enseigner des connaissances spécifiques à des cohortes étroites pour des fins précises. Del'autre côté du Rhin au contraire, lestechnische U niversitäten sont les ultimes héritières d'institutions d'enseignement technique qui avaient comme référence permanente les universités autonomes dont le modèle avait été établi au début du XIX ${ }^{e}$ siècle, c'est-à-dire de puissantes structures à la vocation culturelle affirmée, dont le corps professoral était reconnu comme l'élite de la nation : les technische Hochschulen ont mis en œuvre un processus de longue haleine pour parvenir, elles aussi, à la dignité et à la reconnaissance universitaire. Dans le cas des trois pays du sud méditerranéen concernés, il ne semble pas y avoir eu de réflexion à propos des modalités françaises d'enseignement technologique supérieur : faut-il conclure à un point aveugle, à une telle imprégnation par le modèle colonial d'origine qu'on ne pouvait même concevoir une alternative? Puisqu'il n'y avait pas, ou si peu, d'écoles d'ingénieurs en place, aurait-on pu imaginer l'élaboration d'autres structures?

Pour terminer (provisoirement...) sur la question de la formation, il faudrait finalement examiner ce qu'il en est des professeurs. Dans les études sur les ingénieurs, ils sont souvent les oubliés. Pourtant leur rôle est fondamental. Classiquement, les professeurs des écoles d'ingénieurs sont considérés comme se situant au cœur de trois dynamiques : tout d'abord, la formation des spécialistes en technologie. C'est l'activité la plus reconnue, celle qui consiste à la fois à faire apprendre des contenus de connaissance, mais aussi initier à des méthodes et spécialement celle d'apprendre à apprendre, en tout temps et à être capable de restituer cet ensemble de savoirs de manière opérationnelle. Ce 
n'est toutefois pas la seule tâche des professeurs. La seconde est celle de la recherche, généralement à des fins d'application, proche ou à moyen terme. Une telle activité implique le plus souvent des contacts et échanges avec des homologues, suscitant des coopérations et conduisant à des publications et prises de brevet. Les résultats de cette activité amènent aussi de la matière nouvelle aux cours et permettent de diffuser des connaissances inédites qui donnent ainsi aux jeunes ingénieurs des atouts pour se placer sur le marché du travail, car ces derniers apportent aux sociétés qui les engagent des données originales que n'ont pas ou n'ont plus ceux qui sont en poste depuis plusieurs années. Troisième fonction enfin, celle du conseil aux entreprises, utile aux deux parties. Car si le professeur amène des informations pertinentes, si son regard distancié permet de désigner les problèmes cachés et de proposer des solutions, il retire aussi de ses observations des éléments qui nourriront tant sa démarche pédagogique que sa pratique de recherche. C'est en raison de cette triple démarche, chacun des éléments renforçant les deux autres, que le professeur peut être considéré comme un des producteurs essentiels de la culture technique moderne.

Un tel profil est évidemment idéal-typique. Dans la réalité, il va de soi que les professeurs ne sont pas calqués sur le même modèle. Certains ne font pas ou font peu de recherche, d'autres ont rarement des contacts avec le milieu industriel et les investissements dans I'enseignement nesont pas identiques. Néanmoins, si l'on considère l'équipe pédagogique dans son ensemble, on peut lui appliquer alors le terme $d^{\prime}$ ' « intellectuel collectif » et envisager quels sont ses engagements dans ses trois fonctions. Une telle étude est particulièrement éclairante à certains moments clés, comme par exemple la création d'un établissement, une évolution économique notable dans le pays ou, sur le plan scientifique, l'émergence d'une nouvelle discipline qui remet en cause les paradigmes les mieux établis. Sur le plan historique, par exemple, la naissance de l'électricité comme discipline spécifique, à la fois ouvrant un champ scientifique nouveau, porteuse de technologies d'avant-garde, et générant un secteur industriel autonome, a amené la constitution d'un corps professoral particulier qui participait de cette dynamique sur la base des trois fonctions succinctement décrites ci-dessus. Des études de cas dans chacun des trois pays apporteraient des données tout à fait utiles pour l'analyse des ingénieurs au Maghreb. On pourrait ainsi analyser le mode de fonctionnement du corps professoral au moment de la création et dans les débuts des établissements les plus emblématiques de chaque Etat, mais aussi l'examiner aujourd'hui, dans une période où I'on constate une inflexion marquée vers une ouverture de chacune des économies 
nationales en direction du secteur privé, mais aussi où les difficultés d'emploi d'une partie des jeunes ingénieurs interpelle directement le système de formation. Une autre piste d'étude serait la question soulevée par M ohamed Benguerna à propos des premières années de l'Ecole polytechnique d'Alger : cet établissement a fonctionné avec l'aide de professeurs « intérimaires » pour compléter les équipes et pallier le manque d'enseignants autochtones, qu'il s'agisse de coopérants français, d'un staff composé avec l'appui de I'UNESCO ou encore de professeurs des pays de l'Est. Cette expérience n'est pas unique, on la retrouve dans les trois pays. En particulier, on sait que les enseignants des pays de la zone d'influence soviétique, et notamment russes, sont venus en quantité significative encadrer des étudiants en sciences et technologie, dans nombre d'établissements - au moins en Algérie et en Tunisie -, mais les conditions de leur arrivée, leur mode d'insertion, la nature de leurs travaux, la durée de leur séjour et d'autres données encore (quelle langue d'enseignement, par exemple) n'ont, semble-t-il, jamais fait l'objet d'études spécifiques. Un tel phénomène n'est pourtant pas anecdotique, il s'agit pour les écoles d'expériences fondatrices qui marquent durablement leur culture.

\section{Pour ne pas Conclure : Les PeRspectives ReCherches}

On pourrait évidemment multiplier les angles d'analyse sur les ingénieurs. Encore faudrait-il pouvoir ensuite disposer des forces intellectuelles pour mettre en œuvre les projets de recherche sur les différentes thématiques proposées. Néanmoins, pour terminer, je souhaiterais évoquer brièvement deux axes qui ont fait l'objet de débats au sein du collectif de recherche : le problème du chômage des ingénieurs; la question des idéologies et des systèmes de valeurs.

Nous avons peu d'indications sur le chômage des ingénieurs au Maghreb. Et pourtant, de l'avis de tous les collègues engagés dans la recherche sur Ingénieurs et société, c'est une des questions les plus prégnantes de ce programme et socialement les plus graves qu'il faille considérer. Plusieurs pistes d'explication ont été proposées. La crise économique, plus ou moins forte selon les périodes, qui dure depuis vingts ans et qui touche particulièrement les jeunes pays est une des raisons de fond de cet état de fait. La fin de la problématique développementaliste conçue autour du rôle central d'un Etat tout-puissant, ce qui a amenéà faire des coupes sombres dans des sociétés d'Etat et à faire « maigrir » les administrations techniques dans lesquelles on trouvait nombre d'ingénieurs, en est une autre qui ne s'oppose pas à la première. L'adéquation problématique entre les programmes de formation et les nouvelles demandes des firmes 
privées constitue également un des motifs avancés au chômage ou en tout cas au sousemploi d'une partie du groupe ingénieurs.

Pour avancer dans cette analyse, le rassemblement de données de cadrage statistiques serait indispensable et là encore l'harmonisation des nomenclatures pourrait permettre de mesurer l'amplitude du phénomène et sa conjonction entre les trois pays. II n'est pas certain que la tâche soit aisée, les données pouvant être mouvantes du fait de changements dans les catégories rendant impossible l'établissement de séries, mais aussi parce qu'étant à base déclarative, ces informations sont susceptibles d'être biaisées par les réticences des intéressés à se déclarer chômeurs. Mais au-delà, des enquêtes qualitatives devraient permettre de comprendre le vécu de ces diplômés « exclus » du travail. Pour ne pas confondre des situations qui sont detype différent, on pourrait concevoir l'examen de trois sous-populations :

- Les jeunes diplômés, sortis tout droit de leur établissement de formation et qui n'ont pas encore trouvé d'emploi. Quelles activités ont-ils ? Quelle vie personnelle (cohabitation familiale forcée ?) ? Quelles perspectives à court, moyen et long terme? (reprise d'études, reconversion, création d'entreprise, petits boulots...).

- Les ingénieurs en cours d'emploi qui ont été licenciés. Dans quel cadre et dans quelles conditions ? Quelles perspectives d'avenir ? Y a-t-il une distinction entre ingénieurs diplômés et cadres techniques d'entreprise pour retrouver un emploi (effet diplôme) ou faut-il s'appuyer sur les relations (effet réseau).

- Les ingénieurs en fin de parcours professionnel. En France, une des modalités pour « soulager » les entreprises d'une partie de leur main-d'œuvre a consistéà offrir des conditions intéressantes de départ, y compris sous forme de licenciement économique avec prime de départ, aux employés les plus âgés. II s'agit donc de mise en retraite déguisée. Trouve-t-on ou a-t-on trouvé des situations similaires au Maghreb ? Mais il y a aussi de véritables licenciements avec une difficulté spécifique liée à l'âge - ce qui pose une question intéressante : à quel âge devient-on «trop » vieux, c'est-à-dire inemployable, et quels sont les critères du « trop » âgé ?

On le voit, il ne s'agit là que d'une première approche. Une véritable étude supposerait l'établissement d'une problématique plus élaborée.

Le problème des idéologies et des systèmes de valeurs est également une branche importante de l'analyse du groupe des ingénieurs. Elle est ou plutôt elle devrait être, car cet aspect n'est le plus souvent qu'aperçu quand il n'est pas tout simplement ignoré. Or, 
cette dimension axiologique est un moteur de ces acteurs dans leurs tâches professionnelles comme dans leur rôle dans la société, même s'ils n'en ont pas toujours une conscience très élaborée. En France, depuis le saint-simonisme du début du XIX siècle jusqu'aux réflexions contemporaines sur l'éthique de l'ingénieur en passant par les propositions sur le rôle social de l'ingénieur à partir de la seconde industrialisation, le planisme des années vingt et la dynamique du christianisme social des années trente, ces questions n'ont cessé d'interpeller les ingénieurs et de les mobiliser, même à leur corps défendant. Les ingénieurs d'aujourd'hui sont les héritiers de ces différentes théories et/ ou croyances qui constituent un ensemble composite d'idées plus ou moins applicables aux données contemporaines.

II ne semble pas que cette thématique ait fait l'objet de recherches spécifiques dans I'aire culturelle arabo-musulmane sauf à considérer l'apport important de Nilüfer Göle qui traite du monde spécifique turc et dont les travaux sont déjà anciens ${ }^{4}$. Or dans ce domaine, les idées évoluent vite. La dynamique del'islamisme est évidemment sur toutes les lèvres, mêmes'il ne peut êtrequestion de neconsidérer quecette seuleidéologie. C'est pourquoi des recherches précises avec des hypothèses argumentées devraient être entreprises, peut-être à l'issue d'un séminaire consacréà cette question, avec des apports de différents pays du Sud comme du Nord.

A l'issue de cette brève énumération des axes de recherche potentiels sur les ingénieurs du Maghreb, on voit que sur la base solide du travail accompli dans le cadre du programme de I'IRMC, il y a encore matière à investigation et mise en œuvre d'un nouveau projet collectif de recherche pour les années à venir.

\section{N OTES}

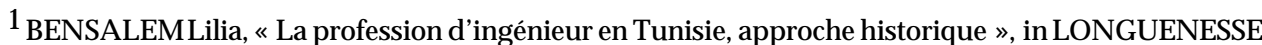
Elisabeth (dir.), Bâtisseurs et Bureaucrates. Ingénieurs et société au M aghreb et au M oyen-O rient, Lyon, Maison de l'Orient méditerranéen, 1990, pp. 81-94.

2 PERENNES Jean-Jacques, «Les ingénieurs et la politique hydro-agricole au Maroc », in LONGUENESSE Elisabeth (dir.), ibid., pp. 215-230.

${ }^{3}$ BOURDIEU Pierre, La noblesse d'Etat. Grandes écoles et esprit de corps, Paris, Editions de Minuit, 1989, p. 188 et pp. 198-199.

${ }^{4}$ GÖLE Nilüfer, Les ingénieurs turcs : avant-garde révolutionnaire ou élite modernisatrice ? thèse sous la direction d'Alain Touraine, Paris, EHESS, 1982. Cette thèse n'a pas été publiée. Un résumé du travail de cet auteur est donnéin LONGUENESSE Elisabeth, op.cit., «Entre le "gauchisme" et l' "islamisme" : l'émergence del'idéologie techniciste en Turquie », pp. 309-320. 

Chapitre 1

\section{SYSTÈMES DE FORMATION}



Le modèle de formation en question 



\title{
La formation des ingénieurs par le système d'enseignement supérieur au $M$ aroc
}

\author{
Kamel MELLAKH
}

Depuis les années 1980, d'importantes transformations sont intervenues dans le système d'enseignement supérieur marocain, touchant autant les modalités d'organisation scolaire que la nature de la demande sociale. La croissance des effectifs étudiants, la diversification des filières d'étude, la décentralisation des pôles de formation et l'accélération du processus de privatisation de l'enseignement supérieur ont donné lieu au développement d'un espace de formation supérieur hétérogène, disparate et hiérarchisé scolairement et socialement. Dans un contexte de « massification » de I'enseignement et de crise de l'université publique (crise de financement, chômage des diplômés, etc.), de nouvelles filières élitistes sont apparues en dehors des anciens lieux de formation (facultés, écoles publiques, etc.) renforçant les mécanismes scolaires de la sélection sociale. Ces évolutions apportent de profondes modifications au modèle national d'enseignement supérieur, explicitement nommé au lendemain de l'indépendance « système de formation des cadres ». Dans ce système, les écoles nationales d'ingénieurs ont été destinées à jouer un rôle prépondérant.

\section{LA FORMATION DES CADRES : STRATÉGIE VOLONTARISTE ET ORGANISATION SÉLECTIVE}

Les politiques publiques en matière de formation au Maroc se sont articulées au lendemain de l'indépendance politique, en 1956, autour de mots d'ordre nationalistes : I'organisation d'une éducation authentiquement nationale, reval orisant la langue arabe et permettant à l'ensemble des jeunes autochtones d'accéder à l'école. L'expansion de la scolarisation, puis la «massification » des effectifs de l'enseignement à tous les niveaux ont résulté d'une telle politique ${ }^{1}$. Dès la première année de 1956 à 1957, les effectifs scolaires sont passés de 415151 à 625 659. Par un dahir (décret royal) de novembre 1963, 
l'enseignement est déclaré obligatoire pour tous les enfants âgés de 7 à 13 ans. Dix ans après I'indépendance, les effectifs avaient doublé, atteignant le chiffre de 1321786 élèves.

Cette progression rapide survenue en un temps relativement court n'allait pas sans remettre en cause la politique scolaire suivie jusqu'alors. Si les principaux slogans nationalistes restaient légitimes tels que I'unification, l'arabisation, et la « marocanisation », le contrôle des flux s'imposa. La généralisation de l'enseignement comme utopie nationale, celle d'une école accessible pour tous, devait changer de sens. La démocratisation de l'enseignement n'était plus à l'ordre du jour. L'objectif assigné au développement de l'éducation visait plutôt à favoriser la consolidation del'Etat moderne. Ainsi, les efforts à fournir en matière d'éducation avaient pour but de former les cadres techniques et administratifs nécessaires pour prendre la relève des coopérants. La « marocanisation » de l'encadrement des secteurs clefs du développement (économie, éducation, agriculture, santé...) était devenu une priorité des politiques publiques de planification de l'éducation : les besoins en cadres nationaux capables de restructurer l'économie et les secteurs sociaux, dans le sens de l'intérêt national étaient souvent évoqués pour justifier de telles politiques. Mais il nous paraitt toutefois que les exigences del'édification de bases institutionnelles pour la monarchie, comme celles de moderniser le $M$ akhzen par un personnel cadre hautement qualifié, ont rendu inéluctable la mise en place d'un réseau national d'écoles et d'instituts de formation supérieure. A cet égard, les écoles publiques d'ingénieurs ont bénéficié d'une attention particulière. Les ingénieurs étaient alors considérés comme les détenteurs des compétences techniques indispensables à la mise en œuvre des projets de développement : ces derniers se sont multipliés dans divers domaines au cours des années 1960 et 1970 (agriculture, bâtiment, architecture, travaux publics, urbanisme, etc.). En deux décennies ont été créées l'Ecole Mohammadia d'ingénieurs (EMI) (1960), I'Institut national de statistiques et d'économie appliquée (INSEA) (1961), I'Institut national des postes et télécommunications (INPT) (1961), I'Institut agronomique et vétérinaireH assan II (IAV) (1966), I'Ecolenational eforestière des ingénieurs (ENFI) (1968), I'Ecole Hassania des travaux publics (EHTP) (1971), I'Ecole nationale de l'industrie minérale (ENIM) (1972), I'Ecole nationale d'architecture (ENA) (1980) et I'Ecole nationale supérieure d'électricité et de mécanique (ENSEM) (1984).

En somme, la multiplication des écoles d'ingénieurs s'est opérée selon une stratégie de formation pragmatique visant à satisfaire les besoins sectoriels des différents départements de l'Etat. Or, il est significatif que la plupart de ses écoles ont été placées sous la tutelle des départements ministériels directement concernés par la formation. 
Tableau 1. Les écoles publiques d'ingénieurs et leur ministère de tutelle au $M$ aroc

\begin{tabular}{|l|l|}
\hline \multicolumn{1}{|c|}{ Ecoles } & \multicolumn{1}{|c|}{ M inistère de tutel le } \\
\hline Institut agronomique et vétérinaire Hassan II à Rabat & Agriculture et développement rural \\
\hline Ecole nationale d'agriculture à Meknès & Agriculture et développement rural \\
\hline Ecole nationale forestière d'ingénieurs à Salé & Agriculture et développement rural \\
\hline Ecole Hassania des travaux publics à Casablanca & Travaux publics \\
\hline Ecole nationale de l'industrie minérale à Rabat & Énergie et Mine \\
\hline $\begin{array}{l}\text { Institut national des statistiques et d'économie } \\
\text { appliquéeà Rabat }\end{array}$ & Plan et prévision économique \\
\hline Ecole Mohammadia des ingénieurs à Rabat & Enseignement supérieur \\
\hline $\begin{array}{l}\text { Ecole nationale supérieure d'électricité et de } \\
\text { mécaniqueà Casablanca }\end{array}$ & Enseignement supérieur \\
\hline $\begin{array}{l}\text { Institut national des postes et télécommunications à } \\
\text { Rabat }\end{array}$ & Postes et Télécommunications \\
\hline Ecole nationale d'architecture à Rabat & Habitat \\
\hline $\begin{array}{l}\text { Ecole nationalesupérieure des arts et métiers à } \\
\text { Meknès }\end{array}$ & Enseignement supérieur \\
\hline Ecole nationale des sciences appliquées à Tanger & Enseignement supérieur \\
\hline
\end{tabular}

Ces écoles ont tendu à acquérir une place à part dans le système d'enseignement supérieur. En marge des universités destinées à accueillir les effectifs de lycéens bacheliers en constante progression ${ }^{2}$, elles se sont rapidement instituées comme un espace d'excellence scolaire, au sein d'un système éducatif fortement sélectif et hiérarchisé. En 1985, les autorités en charge de l'éducation décidèrent de créer un cycle de classes préparatoires aux grandes écoles d'ingénieurs, suivant en la matière l'exemple français. Ces classes préparatoires furent ouvertes dans les différentes régions du royaume (Agadir, Casablanca, Fès, Marrakech, Mohammedia, Oujda, Rabat, Tanger). Un concours national fut organisé pour y accéder. Les élèves admis, «noyau dur » del'élite scolaire, firent l'objet d'une attention particulière.

A la différence des étudiants des universités, cetteélite a relativement mieux profité de l'infrastructure scolaire, de l'encadrement pédagogique, du système de bourses parce qu'ellea bénéficié, en outre, d'un système de formation et d'évaluation rigoureux à faible déperdition. Dans les années 1960 et 1970, l'Etat a mis en place, au profit de l'élève ingénieur, un système de motivation lui garantissant de réels avantages scolaires et 
sociaux. Il lui était attribué, par exemple, un pré-salaire pendant sa formation, ou encore pour les admis la signature d'un contrat de travail d'une durée minimale de huit ans. A la sortie de la formation, les besoins étaient tels que l'embauche était quasi automatique par le ministère de tutelle de l'institut de formation. Ce dispositif suscita un engouement dans les milieux populaires. On a pu voir combien la mobilisation familiale était forte autour de l'accès au diplôme d'ingénieur, les projets de mobilité sociale étant de plus en plus associés à la réussite par le diplôme au lendemain de l'indépendance 3 .

Durant les deux décennies suivantes, les études supérieures, et en particulier les formations scientifiques et techniques, répondent à de fortes demandes sociales : celles-ci conçoivent, en effet, I'offre publique de formation supérieure comme un système de qualification (certificats et diplômes) garantissant des avantages sociaux (accès au fonctionnariat, promotion sociale, etc.). Le diplôme d'ingénieur apparaît désormais, dans l'imaginaire professionnel des lycéens et de leurs familles, comme un mythe mobilisateur ${ }^{4}$. Pourtant, l'accès aux écoles d'ingénieurs demeure extrêmement contrôlé. Le système de sélection s'est même renforcé, ces dernières années, pour contrôler les flux des élèves et gérer les effectifs qui se sont sensi blement accrus.

\section{LES LAURÉATS DES ÉCOLES D'INGÉNIEURS : CROISSANCE ET DISPARITÉ}

A la veille de l'indépendance du Maroc, les effectifs d'ingénieurs étaient réduits. En 1955, le pays en comptait à peine trente, presque tous formés à l'étranger. Cette génération de pionniers, très vite placée aux commandes des services techniques de I'Etat, s'investit al ors notablement dans la mise en place d'une planification nationale de la formation scientifique et technique ${ }^{5}$ : Ils organisèrent, en parallèle, des colloques pour réfléchir sur le métier d'ingénieur et sur l'amélioration de la formation. II s'agissait de mettre en place et de renforcer un système national de formation de cadres techniques répondant aux besoins des administrations et des différents appareils d'exécution ou de production. Dans cette conjoncture, les candidatures au concours d'entrée dans les écoles d'ingénieurs se sont accrues rapidement : la participation aux différentes écoles nationales d'ingénieurs est passée de 242 à 977 candidats entre 1987 et 1996. De la même façon, les effectifs des écoles d'ingénieurs et de formation de cadres continuent d'augmenter depuis les années 1980. 
Tableau 2. Evolution des candidats inscrits et admis aux concours communs d'accès aux écoles nationales d'ingénieurs

\begin{tabular}{|c|c|c|}
\hline Année & Inscrits & Admis \\
\hline 1987 & 242 & 192 \\
\hline 1992 & 713 & 621 \\
\hline 1995 & 983 & 700 \\
\hline 1996 & 977 & 734 \\
\hline
\end{tabular}

Source : ministère de l'Enseignement supérieur et de la Formation des Cadres

Depuis les années 1980, les effectifs des écoles d'ingénieurs ont continué d'augmenter. Cette croissance est la marque des fortes pressions sociales s'exerçant sur ces écoles. II n'est pas aisé d'évaluer l'évolution de ces effectifs en fonction des différences sociales. N ous ne disposons pas de données chiffrées exhaustives indiquant précisément les origines sociales des élèves-ingénieurs. Toutefois, il semble que le recrutement social de ces écoles tend de plus en plus à se diversifier. Certes, ce sont les familles « aisées » et «moyennes » qui ont pendant longtemps pu se mobiliser pour que leurs enfants adhèrent à leur vision promotionnelle du devenir social. Néanmoins, des élèves issus de familles aux revenus plus modestes parviennent à surmonter la double sélection scolaire et sociale, contribuant ainsi à augmenter les effectifs de cadres en formation.

Dans les années 1980, le rythme de croissance annuelle des effectifs de ces écoles est de $26 \%$, alors que celui del'enseignement supérieur est de $14,5 \%$. Les flux des ingénieurs diplômés de l'étranger (notamment de France) ont aussi contribué à accentuer cette tendance. A la fin des années 1980, 23,3\% des ingénieurs ont été formés à l'étranger, contre un peu plus des trois-quarts formés au Maroc. En 1992, le nombre d'ingénieurs diplômés s'élevait à 17500 (dont 4500 formés à l'étranger). A ujourd'hui, les promotions comptent (toutes spécialités confondues) environ 1000 lauréats par an. L'accroissement du nombre des diplômés issus d'écoles nationales d'ingénieurs a été rapide. L'exemple de l'Ecole M ohammedia des ingénieurs (EMI) est symptomatique à cet égard. Le nombre des lauréats y a connu une croissance continue passant de 34 en 1964 (année de la sortie de la première promotion) à 65 en 1978. Les promotions des années 1980 comptaient chacune près de 100 diplômés et celle de 1996, 205 lauréats. 
Tableau 3. Evolution des lauréats des écoles publiques de formation des cadres scientifiques et techniques

\begin{tabular}{|c|c|}
\hline Année & Lauréats \\
\hline $1986 / 87$ & 1246 \\
\hline $1991 / 92$ & 1557 \\
\hline $1994 / 95$ & 1329 \\
\hline $1995 / 96$ & 1522 \\
\hline
\end{tabular}

Source: ministère de l'Enseignement supérieur et de la Formation des cadres

La croissance du nombre des diplômés, issus du système national de formation des ingénieurs, ne peut cependant pas masquer les distorsions que l'on constate, selon les sexes et les domaines d'étude. Bien que minoritaires dans les formations d'ingénieurs, la présence des femmes s'affirme. Leur place est passée de 1,8 \% en 1975 à 15,6 \% en 1990 du total des diplômés ; elle est de $20 \%$ en 1996.

En ce qui concerne les domaines de formation, les spécialités relevant du domaine agricole demeurent importantes. Un réseau constitué de trois établissements (Institut agronomique et vétérinai re H assan II de Rabat, Ecole national e d'agriculture de Meknès, Ecole nationale forestière d'ingénieurs de Salé) a été mis en place pour accueillir chaque année 2800 élèves-ingénieurs destinés principalement à intégrer des emplois publics (administrations centrales, directions provinciales d'agriculture, offices de mise en valeur agricole...). Les ingénieurs agronomes-forestiers représentent au début des années 1990 $46 \%$ del'ensemble des ingénieurs et pour $94,7 \%$ d'entre eux sont employés par le secteur public : ils constituent bien plus que d'autres le corps d'ingénieurs d'Etat par excellence. Figures emblématiques des politiques de mise en valeur agricole au lendemain de I'indépendance, ils étaient perçus comme des acteurs centraux du développement ${ }^{6}$. Aujourd'hui, dans l'actuel contexte de libéralisation économique, ces ingénieurs ont perdu leur aura et sont frappés de plein fouet par les problèmes de formation, les difficultés d'accès à l'emploi et la dégradation de la situation du travail dans la fonction publique?. 


\section{CRISE dU MOdèle ÉTATISTE DE FORMATION DES INGÉNIEURS ET RECOMPOSITION DU SYSTÈME} D'ENSEIGNEMENT SUPÉRIEUR

Des changements caractérisent l'évolution du système de formation des cadres techniques au Maroc depuis que le problème du chômage des cadres a pris les allures d'une « crise socio-politique $»^{8}$. Au regard de la multiplication des mobilisations collectives des diplômés chômeurs (manifestations, pétitions, sit-in, grèves de la faim...), force est de constater que la question des compétences nationales ne se pose plus en terme de pénurie.

La principale question aujourd'hui est celle des implications scolaires, professionnelles et sociales des arbitrages imposés par le nouveau contexte de la transition libérale. Le développement des logiques privatives dans le champ de la formation des cadres est significatif à cet égard. Nous formulons ici I'hypothèse que le dysfonctionnement structurel du système public de formation supérieure, tout comme son incapacité accrue à répondre aux attentes scolaires et sociales de certaines catégories d'élèves et de leurs familles, aurait rendu inéluctable l'évolution différenciée d'une demande sociale d'acteurs privés en matière de formation et de qualification supérieure. Le triomphe des logiques privatives dans le champ de l'enseignement supérieur dans les années 1990 est une remise en question des bases classiques d'un système scolaire public « à faible rendement interne et externe ». De nouveaux clivages scolaires apparaissent alors même que, d'une manière progressive et irréversible, le système d'enseignement supérieur se recompose selon trois axes :

- un enseignement universitaire de masse (faculté des lettres, des sciences et de droit) à fort recrutement populaire;

- un enseignement supérieur privé à géométrie scolaire et sociale variable selon le volume du capital économique imposéà l'entrée des écoles ;

- un réseau d'écoles publiques de formation des cadres (y compris les écoles d'ingénieurs).

Plusieurs indices permettent de suivre les nouveaux rapports de force qui s'instaurent entre ces différentes composantes du système de formation en particulier entre les écoles publiques d'ingénieurs et les grandes écoles supérieures privées (HEM, HEC...). Si le chômage des diplômés et la crise du financement de l'éducation frappent de plein fouet les facultés, les écoles publiques d'ingénieurs et de cadres ne sont pas épargnées. Tout en demeurant très sélectives, ces écoles tendent à devenir socialement peu rentables. Les horizons professionnels de leurs lauréats sont de plus en plus bouchés. 
Bien sûr, le titre d'ingénieur continue à jouir d'un certain prestige dans l'imaginaire professionnel des lycéens ${ }^{9}$, mais ce prestige ne compense pas le malaise d'une grande partie des ingénieurs diplômés. A l'euphorie des années 1960, lors de l'édification des premières grandes écoles nationales d'ingénieurs et de formation de cadres scientifiques, a succédé l'inquiétude dès le début des années 1980. En 1984, les ingénieurs organisent une première rencontre sur le thème de la formation ${ }^{10}$; pour tenter $d^{\prime}$ 'apporter une réponse au chômage des cadres techniques dont les premiers signes se manifestent, ils réclament une meilleure adaptation du cursus de formation. L'actualisation des contenus des programmes et l'introduction de nouvelles disciplines (gestion, organisation, management...) sont au centre des revendications des ingénieurs. Leurs écoles ont alors du mal à se maintenir comme espace d'excellence scolaire dans un contexte de crise de l'université publique et de transition libérale. A l'inverse, les grandes écoles privées apparaissent comme des vecteurs d'innovation pédagogique et suscitent l'intérêt des instances en charge des reformes éducatives (introduction dans les écoles d'ingénieurs de cycles de formation en communication et management, création d'écoles nationales de gestion et de commerce à Settat, Tanger et Agadir...). Les écoles privées, créées dans les années 1990, apparaissent plus attrayantes. Elles proposent aux étudiants des formations en coopération avec les universités et les écoles européennes et américaines. Les formations y sont principalement axées sur la gestion, le management, le commerce, le marketing et l'informatique. Les outils pédagogiques utilisés sont interactifs et les stages professionnels qui sont intégrés dans lecursus de l'enseignement permettent detisser des liens entre les milieux professionnels et les étudiants. Enfin, les lauréats des écoles privées, destinés le plus souvent à devenir des cadres commerciaux, analystes financiers ou gestionnaires paraissent mieux armés que les ingénieurs pour affronter les mutations du marché de l'emploi où initiatives privées, auto-emploi, mise à niveau de l'entreprise etc. sont autant de nouvelles valeurs en vogue. Toutefois, ces écoles supérieures privées, en dépit de leur réputation, sont toutes confrontées à une situation paradoxale : l'autorisation d'ouverture délivrée par le ministère concerné n'implique pas I'homologation des titres scolaires. Elles se trouvent, au regard de l'Etat, incapables de produire des diplômes légitimes. Cette situation conduit donc des établissements privés à revendiquer, avec acharnement, la reconnaissance des pouvoirs publics ${ }^{11}$. Or, ces derniers ne semblent pas prêts à répondre favorablement à leur attente, car ils cherchent plutôt à entretenir une certaine « culture publique » en matière de formation de cadres. Aussi, serait-ils instructif d'observer selon quelles modalités le pouvoir éducationnel 
cherche à redéfinir l'espace de formation des cadres et particulièrement celui des formations d'ingénieurs dans un contexte de changements accélérés des val eurs scolaires et socioprofessionnelles.

\section{N OTES}

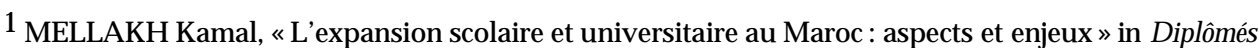
maghrébins d'ici et d'ailleurs : trajectoires sociales et itinéraires migratoires, Ed. CNRS, Paris, 2000.

2 MELLAKH Kamal, «Les candidats marocains au baccalauréat : sur la route de l'université? », contribution au bulletin de liaison du programme de recherche flux et gestion des compétences intellectuelles dans les échanges euro-maghrébins, n 1, juillet-août-septembre 1997.

3 IBAAQUIL Larbi, M obilité, classes sociales et passages par l'école au M aroc, doctorat d'Etat de lettres et sciences humaines, université de Paris V 1987, et, du même auteur, L'école marocaine et la compétition sociale, Ed. Babil, Rabat, 1996.

${ }^{4}$ MELLAKH Kamal, Les lycéens et la socialisation au M aroc: enquête auprès des élèves des classes terminales à $\mathrm{R}$ abat, thèse de doctorat en sociologie, université de Provence, 1997.

${ }^{5}$ VERMEREN Pierre, «La formation des élites au Maroc et en Tunisie » contribution au bulletin de liaison du programme derecherche flux et gestion des compétences intellectuelles dans les échanges euro-maghrébins, $\mathrm{n}^{\circ} 2$, octobre-novembre-décembre 1997.

6 PEREN NES Jean-Jacques, « Les ingénieurs et la politique hydro-agricole au Maroc » in Bâtisseurs et bureaucrates. Ingénieurs et société au M aghreb et au M oyen O rient, Ed. Maison de l'Orient méditerranéen, Lyon, 1990.

7 MELLAKH Kamal et LAGDALI Nadia, Les femmes cadres au M inistère de l'A griculture, Rapport d'enquête, Direction des ressources humaines, ministère de l'A griculture, royaume du Maroc, juin 1999.

8 MELLAKH Kamal, «L'expansion scolaire et universitaire au Maroc : aspects et enjeux», op. cit.

9 MELLAKH Kamal, Les lycéens et la socialisation au M aroc: enquête auprès des élèves des classes Terminales à Rabat, op. cit.

10 DA OUD Zakia, «Les ingénieurs marocains aujourd'hui », Lamalif, n 153, février 1984 et «Les ingénieurs et leurs entreprises », Lamalif, $n^{\circ}$ 176, avril 1986.

11 Grazia Scarfo-Ghellab note que contrairement au cas italien où les écoles de gestion cherchent à construire un espace de formation autonome, les écoles de gestion marocaine s'acharnent à obtenir une reconnaissance publique, au risque de se retrouver sous la tutelle de l'Etat. Voir à ce propos SCARFOGHELLAB Grazia, «Privatisation et internationalisation des institutions d'enseignement supérieur : le cas desécoles degestion au Maroc », Information sur les sciences sociales, n³7, 1998 et SCA RFO-GHELLAB Grazia, La transformation du système d'enseignement italien : Ia diffusion des business schools, Paris, Ed. L'Harmattan, 1997. 



\title{
Les ingénieurs tunisiens dans le système éducatif : quel modèle de formation pour les cadres techniques?
}

\author{
Saïd BEN SEDRINE
}

Eric GOBE

A partir des années 1960, la Tunisie, à l'instar des autres Etats du Maghreb indépendants, portée par l'idéologie développementaliste, a initié une politique de «tunisification » de l'encadrement des services techniques. Elle s'est alors engagée dans une stratégie volontariste de formation d'ingénieurs, à long terme, dans le cadre d'une politique de planification du développement économique et de nationalisation des principales entreprises. Aussi le gouvernement tunisien incitait-il ses meilleurs étudiants à entreprendre des études d'ingéni eur ${ }^{1}$.

L'envoi d'étudiants à l'étranger et, plus tardivement, l'accroissement du nombre des écoles tunisiennes d'ingénieurs visaient jusqu'au milieu des années 1980 à satisfaire les besoins en cadres supérieurs des différentes administrations de l'Etat et des sociétés du secteur public. La stratégie de formation mise au service du « développement national » a contribuéà donner ses caractéristiques au « modèle d'organisation du travail technique » tunisien. A la question concernant la formation et la sélection de ses cadres techniques, la Tunisie indépendante a adopté une réponse qui rattachait le pays à ce que certains sociologues anglo-saxons appellent l'« organisation étatique du travail technique ». Cette organisation se caractérise « par une stratification du travail technique explicitement fondée sur les diplômes $»^{2}$ : elle demeure d'autant plus forte en Tunisie que jusqu'en 1990, I'Etat reste le principal recruteur des diverses promotions d'ingénieurs formées dans ses écoles ou à l'étranger.

L'effort de l'Etat s'est traduit, dès 1969, par la création d'établissements nationaux de formation d'ingénieurs. Les premiers responsables de ces nouvelles filières, issus du système d'excellence français, ont mis en place un modèle de formation qui tendait à 
reproduire la dualité caractéristique du système français entre «grandes » et « petites » écoles ou facultés et entre « petite porte» et «grande porte »3. N éanmoins, à la différence des thèses de Pierre Bourdieu, la formulation de cette hypothèse ne préjuge pas d'une éventuelle reproduction des hiérarchies sociales par le système éducatif.

Cette opposition prend en Tunisie une forme spécifique dans la mesure où les filières d'excellence réservées aux meilleurs étudiants sont localisées à l'étranger (en premier lieu, en France, avec les grandes écoles d'ingénieurs). Ce mode de fonctionnement débouche de manière directe sur la question du retour (ou non) des étudiants tunisiens dans leur pays d'origine. La problématique de la « fuite des cerveaux » explique, pour partie, la réforme du cursus d'ingénieur menée par le ministère de l'Education et des sciences, au début de la décennie 1990.

La dual ité originelle se traduit, du fait de cette réforme, par une double séparation au sein du système éducatif : d'une part, entre formation à l'étranger et formation sur place et, d'autre part, entre les établissements d'excellence situés à Tunis et dans sa banlieue résidentielle et les écoles provinciales et/ ou spécialisées (plus particulièrement les instituts délivrant un diplôme d'ingénieur agronome).

\section{U NE POLITIQUE VOLONTARISTE DE FORMATION SCIENTIFIQUE ET TECHNIQUE}

\section{Le développement et la diversification des formations initiales (1969-1990)}

En 1956, au moment de l'Indépendance, la Tunisie ne comptait que 84 ingénieurs dont 48 ingénieurs agronomes : les autres sortaient de l'Ecole polytechnique(5), des Mines de Paris ou de Saint-Etienne (10), de l'Ecole centrale de Paris (1) ; 3 ingénieurs avaient étudié les télécommunications et 4, I'hydraulique à Grenoble. Seulement 2 ingénieurs sortaient des Ponts et Chaussées et 11 étaient issus de l'Ecole spéciale des travaux publics ${ }^{4}$. L'administration du Protectorat n'avait guère intégré dans son administration ces jeunes diplômés formés en France ${ }^{5}$. La plupart d'entre eux ne possédaient que peu d'expérience et $n$ 'avaient occupé aucun poste de responsabilité.

Mais le départ précipité des ingénieurs français peu après la « bataille » de Bizerte en 1961, en paralysant l'économie du pays, constitua « un test révélateur de la dépendance dans laquelle la Tunisie se trouvait pour faire fonctionner les services vitaux du pays ${ }^{6}$. Elle fit prendre conscience aux autorités tunisiennes de l'impérative nécessité de former rapidement des ingénieurs nationaux. 
En août 1961, sur l'initiative du Secrétariat d'Etat à l'Education nationale fut rassemblé dans un «amphi » la quasi-totalité des 620 bacheliers de l'année. Lors de cette réunion, les principaux ingénieurs chefs de service des administrations examinèrent directement avec les candidats à des carrières techniques les orientations concrètes qui s'offraient à eux 7 . Désormais, chaque administration allait suivre individuellement les étudiants boursiers, qu'ils soient inscrits dans des classes préparatoires aux grandes écoles en France, élèves de ces écoles ou encore étudiants de licences ès-sciences. En 1962, plus de 400 boursiers patronnés par les services des Travaux publics et de l'A griculture figuraient dans les classes préparatoires ${ }^{8}$.

A la même époque, le deuxième polytechnicien tunisien, Mokhtar Latiri (X - 1947), à la fois directeur général des ponts et chaussées et des enseignements techniques au ministère de l'Education nationale, avait été chargé de mettre en place une première école d'ingénieurs. Pour fonder la future Ecole nationale d'ingénieurs de Tunis (ENIT), son concepteur s'est adressé tout d'abord aux Etats-Unis dès 1960 pour obtenir le financement de la construction de l'établissement. Mais le Development Loan Fund (DLF), I'agence américaine d'aide au développement, lui avait fait savoir que les Etats-Unis ne financeraient que la construction de l'Institut supérieur de gestion et de la faculté de droit de Tunis ${ }^{9}$. A près le refus américain, le futur directeur de I'EN IT s'était alors rendu en URSS où il réussit à obtenir une aide au financement del'établissement: I'URSS en dessine les plans, fournit le matériel et les engins de construction, soit $50 \%$ du coût de la construction. Pour sa part, la Tunisie investit à hauteur de $40 \%$, les $10 \%$ restants étant pris en charge par des Etats tels que la Suisse et les Etats-Unis ${ }^{10}$. La construction de l'école est achevée en 1968, mais elle n'a pas encore de laboratoires ni de corps professoral. Alors que les nouveaux locaux de la faculté des sciences sont également en voie d'achèvement, le Premier ministre, A hmed Ben Salah, met en place un «tronc commun » de deux ans où sont regroupés les futurs ingénieurs et les étudiants de la faculté des sciences ${ }^{11}$. En 1970, les premiers élèves ingénieurs titulaires du diplôme universitaire d'études supérieures (DUES) sanctionnant le tronc commun sont envoyés dans des écoles françaises d'ingénieurs pour y suivre les enseignements appliqués (plus particulièrement à l'Ecole des mines de Saint-Etienne et à l'Ecole supérieure des travaux publics) ${ }^{12}$.

Dans le même temps, Mokhtar Latiri, nommé directeur de l'ENIT en août 1968, conçoit la structure du cursus de formation de l'école. II institue quatre filières de formation. La première, instaurée dès 1965 , est en fait une voie de sélection sous-traitéeà l'étranger, par de grandes écoles françaises, des universités allemandes et américaines. 
Initialement, la filière $\mathrm{A}$ devait préparer les meilleurs bacheliers scientifiques au titre d'ingénieur-docteur dans le cadre d'un cursus de 8 ans ${ }^{13}$. Entre 1968 et 1975, le directeur de l'ENIT organise la sélection et le départ d'une cinquantaine de lauréats dans les lycées parisiens les plus réputés (lycées Saint-Louis ou Louis Le Grand) ${ }^{14}$. A près I'éviction de Mokhtar Latiri de la direction del'ENIT en 1975, ce processus de sélection pour les classes préparatoires françaises sera pris en charge par une commission du ministère de I'Education nationale.

La filière $B$ forme des « ingénieurs diplômés » en 6 ans. Ce cursus comporte trois cycles de deux ans chacun : sur le modèle de l'Institut national des sciences appliquées (INSA) de Lyon, un cycle préparatoire intégré dispense un enseignement général scientifique, technique et en sciences humaines; un deuxième cycle porte sur l'art de l'ingénieur, le troisième cycle étant consacré à l'une des options de l'art de l'ingénieur ${ }^{15}$.

La troisième filière, dite « $C »$, forme des «ingénieurs techniciens ». Elle propose trois cycles d'études dont les durées sont fixées respectivement à un an, deux ans et un an. Le premier porte sur l'enseignement général scientifique et technique. Le second est consacré à «l'art de l'ingénieur technicien », tandis que le troisième propose une spécialisation. Enfin, la filière $D$ produit des techniciens supérieurs et dispense un enseignement analogue aux actuels IUT français ${ }^{16}$.

Dès la mise en place de l'école, le concepteur de l'ENIT a associé des responsables des grandes écoles françaises à la réflexion sur les orientations pédagogiques de l'établissement ${ }^{17}$. En outre, le corps enseignant de l'écol e est composé en majorité durant la décennie 1970 d'enseignants français et, dans une moindre mesure, d'enseignants soviétiques ${ }^{18}$.

Il importe d'insister sur l'organisation des études de l'EN IT dans la mesure où, de la naissance de l'établissement jusqu'à la réforme du cursus de l'ingéniorat au milieu des années 1990, l'enseignement des autres écoles d'ingénieurs devait être organisé sur le modèle des filières $B$ et $C$.

Parallèlement à la voie des classes préparatoires, les autorités tunisiennes ont négocié des admissions directes dans certaines écoles d'ingénieurs françaises (I'Ecole supérieure des travaux publics, Centrale, les Mines etc.) : tout au long des années 1960 et 1970, de nombreux étudiants tunisiens, titulaires de maîtrises de sciences, ont ainsi pu intégrer sur titre les grandes écoles d'ingénieurs françaises. Les résultats d'une enquête que nous avons conduite auprès d'un échantillon représentatif d'ingénieurs ${ }^{19}$ montrent 
que c'est parmi les cadres techniques formés en France et en Amérique du $\mathrm{N}$ ord que nous trouvons les taux de titulaires de maîtrises les plus importants, soit respectivement $18,3 \%$ et $15,2 \%$. Ceux qui ont suivi leur cursus en France ont obtenu, dans une large majorité, leur maîtrise avant 1980 (38,5 \% avant 1975 et $23,1 \%$ entre 1976 et 1980).

Tableau 1. Taux des ingénieurs diplômés titulaires d'une maîtrise en fonction du pays de formation (en \%)

\begin{tabular}{|c|c|c|c|c|c|c|}
\hline $\begin{array}{c}\text { A mérique du } \\
\text { N ord }\end{array}$ & France & $\begin{array}{c}\text { Monde } \\
\text { arabe }\end{array}$ & $\begin{array}{c}\text { Europe } \\
\text { de l'O uest }\end{array}$ & Tunisie & $\begin{array}{c}\text { Europe } \\
\text { de l'Est }\end{array}$ & Total \\
\hline 15,8 & $\mathbf{1 8 , 3}$ & 7,1 & 5,3 & 1,8 & 3,0 & 9,5 \\
\hline
\end{tabular}

Source: EnquêteS. Ben Sedrine \& E. Gobe, 2000.

Dans l'absolu, les ingénieurs diplômés d'un établissement français représentent avec ceux qui ont été formés en Tunisie plus de $70 \%$ des titulaires d'une maîtrise (respectivement $50 \%$, et $23,7 \%$ ). Ce résultat confirme, pour les cadres techniques diplômés de l'H exagone, l'importance de la voie de l'intégration des écoles d'ingénieurs sur titre (cf. tableau 2).

Tableau 2. Distribution des ingénieurs diplômés titulai res d'une maîtrise en fonction du pays de formation (en \%)

\begin{tabular}{|c|c|c|c|c|c|c|}
\hline Tunisie & France & $\begin{array}{c}\text { Monde } \\
\text { arabe }\end{array}$ & $\begin{array}{c}\text { Amérique } \\
\text { du N ord }\end{array}$ & $\begin{array}{c}\text { Europe } \\
\text { de l'O uest }\end{array}$ & $\begin{array}{c}\text { Europe } \\
\text { de l'Est }\end{array}$ & Total \\
\hline 23,7 & $\mathbf{5 0}$ & 10,5 & 7,9 & 5,3 & 2,6 & 100 \\
\hline
\end{tabular}

Source : EnquêteS. Ben Sedrine \& E. Gobe, 2000.

L'EN IT est demeurée la seuleécole d'ingénieurs (hors agronomie) du pays, jusqu'à la création de l'Ecole nationale des ingénieurs de Gabès en 1975. Toutefois, à partir des années 1980, le développement des formations d'ingénieurs s'accélère.

En deux décennies (1972-1996), le nombre des diplômés formés à l'ENIT, et inscrits au tableau de l'Ordre des ingénieurs, tend à augmenter (voir la courbe de régression linéaire du graphique 1)20. Quant à l'effectif cumulé durant cette période, il atteint les 3211 ingénieurs qui se répartissent entre 2300 « ingénieurs techniciens » et 911 « ingénieurs 
principaux ${ }^{21}$. Toutefois, la baisse du nombre des diplômés entre 1992 et 1996 s'expliquerait aussi par la création d'autres écoles en Tunisie. En effet, afin d'élargir la gamme de spécialités enseignées et de rapprocher les établissements de formation des pôles d'activités industrielles, des écoles d'ingénieurs sont créées sur le modèle de l'EN IT. L'Ecole nationale d'ingénieurs de Gabès (ENIG), en particulier, est conçue pour fournir une main-d'œuvre technique qualifiée au pôle des industries chimiques de la ville du sud tunisien. L'Ecole nationale d'ingénieurs de Sfax (ENIS) est mise en place, en 1983, à la suite de la transformation de la filière ingénieur ouverte en 1975 à la faculté des sciences et techniques de Sfax. En 1984, I'Ecole nationale des sciences de l'informatique (ENSI) vient renforcer la filière d'ingénieur informaticien créée à la faculté des sciences de Tunis, également en 1975. Selon le même principe, l'Ecole nationale d'ingénieurs de Monastir (ENIM) prend la relève dela filière ingénieur ouverte à la faculté des sciences et techniques de Monastir en 1977. En 1990, I'Ecole supérieure des postes et des télécommunications de Tunis (ESPTT) voit le jour en remplacement de l'Ecole des postes : cette dernière comportait une filière de techniciens et une filière d'ingénieurs techniciens 22 .

\section{G raphique 1 : Evolution des diplômés de l'Ecole nationale d'ingénieurs de Tunis}

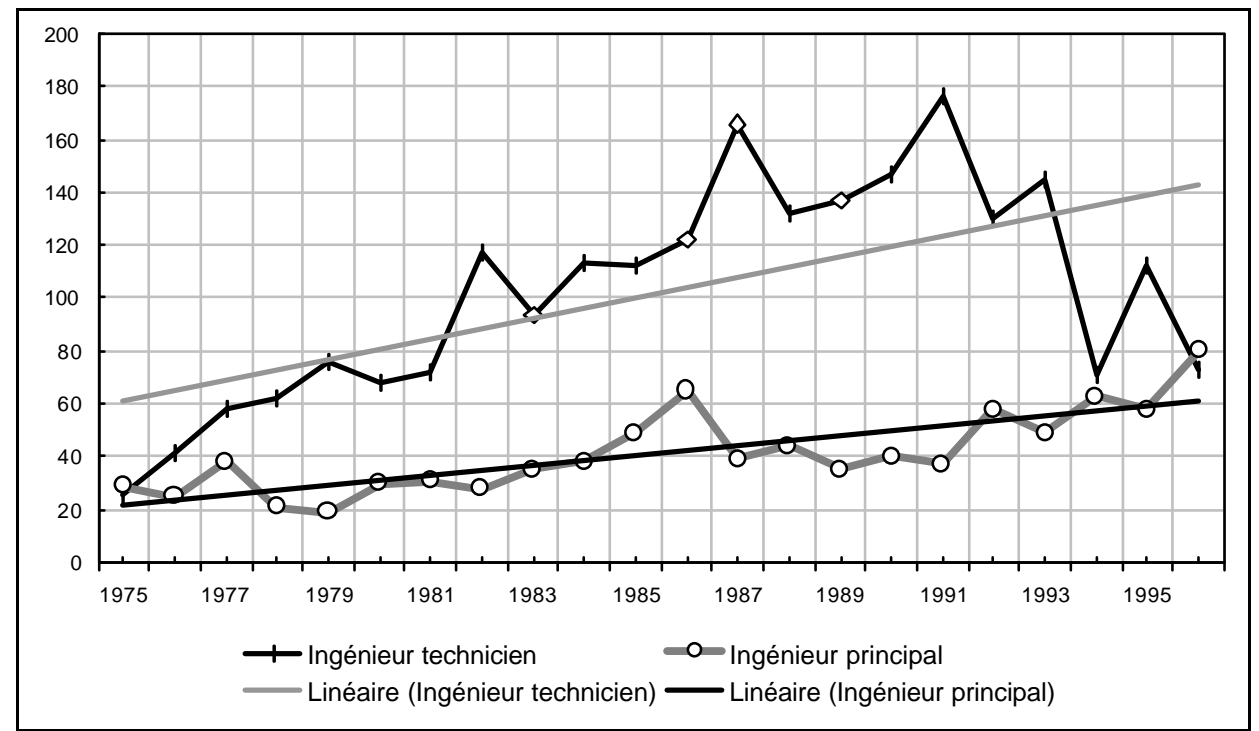

Source: Base de données del'Ordre des ingénieurs(1999), exploitation statistiquepar S. Ben Sedrine\& E. Gobe

Premier constat, les écoles d'ingénieurs qui ont vu le jour pendant les années 1980 ont été créées à partir de départements universitaires. Juridiquement, la plupart des établissements délivrant un diplôme d'ingénieurs fonctionnent sous la tutelle du 
ministère de l'Enseignement supérieur et sont administrativement rattachés à I'Université tunisienne. Les départements universitaires scientifiques qui peuvent dépendre administrativement de la même université se distinguent de ses écoles sur un point essentiel : elles ne délivrent pas le titre scolaire d'ingénieur, à l'exception de trois filières au sein de la faculté des sciences de Tunis.

Cette dernière propose depuis 1979 trois filières de formation d'ingénieurs (en informatique, en géoscience et en chimie analytique). C'est l'administration qui, après avis du Conseil de l'Ordre des ingénieurs, fixe la liste des établissements habilités à délivrer un diplôme d'ingénieur. Les écoles d'ingénieurs agronomes sont sous la cotutelle du ministère de l'Agriculture et de celui de l'Enseignement supérieur qui se contente d'exercer une tutelle pédagogique. II en est de même pour I'ESPTT qui dépend des ministères des Communications et del'Enseignement supérieur. La tutelle exercée par un ministère technique signifie à I'origine que ce dernier, ainsi que les établissements ou entreprises publics qui en dépendent, a vocation à recruter les diplômés de l'école qu'il chapeaute. Si cela est toujours vrai dans le cas du ministère de la Communication, ça l'est beaucoup moins pour celui de l'Agriculture (voir infra). A la grande époque de I'ingénieur d'Etat, l'EN IT avait justement été rattachée en 1971 au Premier ministère pour affirmer sa vocation à former les cadres techniques de l'administration et des entreprises publiques $^{23}$.

\section{Ingénieurs techniciens versus ingénieurs principaux ?}

L'ensemble de ces établissements, tout comme les formations dispensées à l'étranger (notamment en France), était censé répondre aux besoins de l'économie tunisienne en «ingénieurs de conception » (filière $A$ et bac +6 ), ainsi qu'en «ingénieurs de production et de maintenance » (bac +4$)$. Les premiers étaient conçus par le promoteur de l'ENIT comme des cadres techniques capables d'inventer des systèmes et être des experts dans la gestion des organisations, alors que les seconds étaient là pour exploiter les systèmes mis au point par d'autres. La référence utilisée pour illustrer le « rôle » des « ingénieurs techniciens » était emprunté à un exemple français. En effet, ces derniers devaient, selon les propos du premier directeur de l'ENIT, jouer « un rôle anal ogueà cel ui que jouaient autrefois les ingénieurs-maisons qui ont constituél'ossature de firmes comme Citroën et Peugeot $\gg^{24}$. 
Les premiers sortaient de l'école avec le titre d'ingénieur diplômé leur donnant le droit d'entrer dans l'administration comme ingénieur principal, tandis que les seconds étaient titulaires du diplôme d'ingénieur technicien qui leur permettait d'accéder au grade d'ingénieur des travaux de l'Etat dans la fonction publique ${ }^{25}$.

Cette répartition par grade est donc une conséquence de la manière dont a fonctionné le système de formation des ingénieurs en Tunisie jusqu'à la réforme du cursus au milieu des années 1990. Elle est caractéristique d'une certification scolaire qui filtre l'accès à l'encadrement technique et renvoie au «modèle d'organisation étatique du travail technique » précédemment décrit. La nature du diplôme obtenu, non seulement donne la possibilité aux ingénieurs de travailler dans la fonction publique, mais détermine les profils de carrière. Par conséquent, ce modèle d'organisation favorise « des orientations catégorielles fondées sur la défense des titres scolaires ${ }^{26}$. On peut ainsi en conclure que la reconnaissance par l'Etat du statut des diplômes délivrés par les écoles d'ingénieurs (étrangères ou tunisiennes) revêt des enjeux matériels et symboliques très forts. L'histoire et le développement des formations d'ingénieurs agronomes sont à cet égard illustratifs.

\section{Le cas des formations d'ingénieurs agronomes}

Au moment de l'indépendance, l'agriculture présentait un déficit en cadres techniques moins grand que les autres secteurs de l'économie tunisienne. La création en 1898 de l'Ecole coloniale d'agriculture de Tunis (ECAT) dont le rôle consistait à former techniquement les colons destinés à s'installer en Tunisie ou dans les autres pays de I'Union française, a permis à quelques Tunisiens, généralement fils de grands propriétaires terriens, d'y poursuivre leurs études.

A la suite de la proclamation de l'autonomie interne en 1955, le gouvernement tunisien décida de débaptiser l'école et de lui donner le nom d'Ecole supérieure d'agriculture de Tunis (ESAT). Jusqu'en 1962, cette école, placée sous la tutelle du ministère del'Agriculture, n'a pas subi de changements notables, tant du point devue du régime des études (trois ans), que de la composition du corps professoral : les quatre enseignants tunisiens y représentaient une minorité face à douze Français et un Belge27. Entre 1954 et 1964, les ingénieurs diplômés del'ESAT sont contraints d'occuper les postes laissés vacants à la suite du départ des Français. Ce processus s'accélère avec la nationalisation, en mai 1964, des terres des colons. La France retire alors tous ses techniciens du ministère de l'A griculture ${ }^{28}$. 
Les diplômés de cette période accèdent au grade d'ingénieur des travaux de l'Etat dans la fonction publique. Par ailleurs, en vue de former les futurs formateurs de l'Ecole et d'avoir à sa disposition des «cadres de conception », le gouvernement tunisien attribue des bourses aux étudiants désireux de préparer l'entrée aux écoles d'agronomie et de génie rural en France, notamment le concours de l'Institut national agronomique (INA) de Paris-Grignon 29 . En 1963, la direction de l'établissement, devenu Ecole nationale d'agriculture de Tunis (ENSAT), a décidé d'allonger la durée des études à 4 ans (une année préparatoire plus trois ans) dans le souci de former des enseignants et des ingénieurs à part entière. Déjà en 1962, la direction de l'Ecole avait autorisé les lauréats de chaque promotion à poursuivre des études à l'université de Tunis, en vue de compléter leur formation avant de pouvoir s'inscrire en doctorat à l'étranger. Le passage obligé par la faculté des sciences n'était plus exigé pour les diplômés du nouveau régime $(\mathrm{bac}+4)$, candidats à un troisième cycle $\mathrm{e}^{30}$.

A partir de 1965, un troisième cycle de formation est ajouté au sein de l'établissement : il offre alors aux « bons » étudiants du cycle normal (bac +4$)$, porteurs du titre d'ingénieur agricole, la possibilité de suivre un cycle de deux années supplémentaires leur donnant le titre d'ingénieur agronome ${ }^{31}$. Cette réforme n'est pas dénuée d'enjeux symboliques et matériels dans la mesure où l'administration reconnaît à I'ENSAT I'habilitation à délivrer un diplôme accordant à son titulaire l'accès au grade d'ingénieur principal dans la pyramide de la fonction publique, d'une part ; d'autre part, l'Etat montre que l'enseignement agricole dispensé en Tunisie permet d'accéder à des diplômes équivalents à ceux délivrés dans les grandes écoles d'agronomie étrangères. Entre 1965 et 1968, ce cycle d'étude se déroule entièrement hors de Tunisie, faute d'un encadrement suffisant sur place. Mais, à partir de 1968, la première année théorique de ce cycle despécialisation est sous-traitée à I'INA Paris-Grignon dans lecadre d'un accord de coopération entre les deux établissements, tandis que la seconde année d'application a lieu dans un laboratoire relevant de l'ENSAT ou d'un autre organisme de recherche ${ }^{32}$.

La fin de cette décennie constitue cependant une période de turbulence pour l'établissement. L'accentuation du dirigisme économique, appliqué depuis le début des années 1960, débouche sur une réforme de l'Ecole. Cette dernière se doit de participer à I'œuvre de collectivisation des terres qui se généralise début 1969. Le gouvernement tunisien lui fixe un double objectif : non seulement alimenter en cadres techniques les organismes chargés de la gestion des terres domaniales héritées de la colonisation ; mais aussi former les dirigeants des coopératives de production de grande taille constituées à 
partir des exploitations privées expropriées 33 . A la rentrée universitaire 1968, l'ENSAT se trouve intégrée à l'enseignement supérieur sous le nom de facul té d'agronomie de Tunis. Cette mesure remet en cause le système de formation qui venait d'être mis en place : les étudiants orientés vers la faculté d'agronomie sont ainsi appelés à passer deux ans à la faculté des sciences de Tunis, avant de rejoindre l'Ecole pour y suivre une formation technique de deux années supplémentaires.

L'abandon de la politique de collectivisation, fin 1969, a pour conséquence de replacer l'établissement sous la tutelle du ministère de l'Agriculture. L'Ecole troque le nom de faculté d'agronomie contre celui d'Institut national agronomique de Tunis. Ces rattachements successifs ouvrent une période d'incertitude concernant le régime des études. Un décret de mai 1971 ramènel'Ecole au statu quo ante avec deux diplômes. Celui d'ingénieur agricole nécessite quatre années d'études dont deux préparatoires et deux autres de formation agronomique générale. Celui d'ingénieur agronome est réservé à ceux qui ont été choisis sur dossier par le conseil des professeurs et proposés au ministère de l'A griculture en vue d'une spécialisation de deux ans, soit au total six ans d'étude ${ }^{34}$. Mais certaines questions restent en suspens. Quel doit être le régime de la scolarité pour les étudiants qui ont passé 2 ans à la faculté des sciences ? Et à quel diplôme sont-ils en droit de prétendre?

Des mouvements de contestation et de grève se produisent au sein de l'établissement. Selon le compromis trouvé à la rentrée de 1972, le diplôme d'ingénieur agronome est délivré à ceux qui sont passés par la faculté des sciences après cinq ans ${ }^{35}$ : après leur tronc commun, il est prévu qu'ils fassent deux ans d'études agronomiques et une année de complément de formation et de stage. Néanmoins, ces étudiants ne pourront être recrutés par l'administration qu'au grade d'ingénieur des travaux de l'Etat, on leur accorde tout de même, lors de leur nomination, une bonification d'anciennetée ${ }^{36}$. Quant à ceux qui souhaitent continuer en troisième cycle de spécialisation, ils doivent réussir un concours.

Ainsi, I'INAT, tout comme l'ENIT, allait former des ingénieurs techniciens et principaux à travers deux filières. Mais à la différence de ce qui se passe dans la toute nouvelle école d'ingénieurs, ces deux filières restent non individualisées. En d'autres termes, nous sommes face à un cursus entièrement intégré où la filière longue (bac +6$)$ constitue le prolongement de la filière moyenne $(\mathrm{bac}+4)$. En outre, I'INAT, qui propose plusieurs spécial ités au niveau du $3^{\circledR}$ cycle, continue d'en sous-traiter à l'étranger jusqu'à la fin des années $1980^{37}$. 
Les ambitieux programmes de développement mis en œuvre à partir du milieu des années 1970 (construction de barrages, aménagement de périmètres irrigués, actions d'intensification de l'élevage...) nécessitent un nombre accru de techniciens et d'ingénieurs que ne peuvent fournir ni I'INAT38 ni les écoles françaises d'agronomie. Pour répondre à cette demande, de nouveaux établissements d'enseignement supérieur agricole sont créés qui ont pour mission de développer des filières spécialisées. Ces dernières sont localisées dans les principales régions de grandes cultures, d'élevage, d'agrumiculture, etc. :

-1972: Ecole supérieure des ingénieurs del'équipement rural (ESIER) de M edjez EI Bab (machinisme et génie rural),

-1975 : Ecole supérieure d'horticulture et d'élevage (ESHE) de Chott Mariem,

-1976 : Ecole supérieure d'agriculture (ESA) de Mateur (élevage),

-1976 : Ecole supérieure d'agriculture (ESA) du Kef (grandes cultures),

-1976 : Ecole supérieure des industries agroalimentaires (ESIA) de Tunis,

- 1981 : Ecole supérieure d'agriculture (ESA) de Mograne (agro-économie) ${ }^{39}$.

Dans un premier temps, seules les écoles de Chott Mariem et de M edjez El Bab sont autorisées à former des ingénieurs techniciens. Les autres instituts suivront à la fin des années 1970 et au début de la décennie 1980. Ce développement institutionnel est la conséquence de la stratégie mise en œuvre par l'Etat tunisien pour remédier à la dépendance de la Tunisie, notamment en produits céréaliers. Pour augmenter la productivité et moderniser l'outil de production, les autorités centrales ont misé sur la formation d'ingénieurs chargés de diffuser leur savoir-faire en milieu rural, ainsi que les techniques adaptées pour atteindre les objectifs fixés par les plans de développement 40 . La recherche de l'autosuffisance alimentaire et le développement des exportations de produits tunisiens compétitifs sur les marchés internationaux et tout particulièrement européen demeurent jusqu'à nos jours les deux principaux axes de la politique tunisienne de développement agricole.

Ce volontarisme politique explique la prédominance des agronomes dans la population des ingénieurs tunisiens : ils en constituent un peu plus du quart ${ }^{41}$. De 1956 à 1998, I'INAT, sous ses différentes appellations, a formé 2546 ingénieurs, alors que les six autres instituts réunis ont, en 25 ans, délivré des diplômes d'ingénieurs à 2197 personnes ${ }^{42}$. 
Les cadres techniques agronomes ont été formés, pour la plupart, en Tunisie (61,9\% des ingénieurs agronomes diplômés de l'échantillon), et, dans une moindre mesure, en France (14,4\%). A l'étranger, la France est le pays qui a accueilli le plus grand nombre d'élèves ingénieurs tunisiens spécialisés en agronomie (cf. tableau 3).

Tableau 3. Distribution des ingénieurs agronomes diplômés en fonction du pays de formation (en \%)

\begin{tabular}{|c|c|c|c|c|c|c|c|}
\hline Tunisie & France & $\begin{array}{c}\text { M onde } \\
\text { arabe }\end{array}$ & $\begin{array}{c}\text { Europe } \\
\text { de l'O uest }\end{array}$ & $\begin{array}{c}\text { A mérique } \\
\text { du N ord }\end{array}$ & $\begin{array}{c}\text { Europe } \\
\text { de l'Est }\end{array}$ & A utre & Total \\
\hline 61,9 & 14,9 & 12,4 & 6,2 & 3,6 & 1 & 0,5 & 100 \\
\hline
\end{tabular}

Source : EnquêteS. Ben Sedrine \& E. Gobe, 2000.

Aussi les ingénieurs agronomes constituent-ils, par excellence, un corps d'ingénieurs d'Etat. Jusqu'en 1986, première année de l'application du plan d'ajustement structurel du FMI, le ministère de l'Agriculture et les organismes apparentés offrent un débouché exclusif aux ingénieurs agronomes. Ceux-ci forment la catégorie de cadres techniques travaillant le plus massivement sous la tutelle de l'Etat, que cela soit dans l'administration (à hauteur de 67,7 \%) ou dans une moindre mesure le secteur public (24,9\%) (cf. tableau 4). Et ce sont également eux qui, dans le cadre d'un marché de l'emploi en voie de libéralisation, rencontrent la plus grande difficulté d'insertion professionnelle ${ }^{43}$.

Tableau 4. D istribution des ingénieurs agronomes selon le statut juridique de l'employeur (en \%)

\begin{tabular}{|c|c|c|c|c|c|c|}
\hline $\begin{array}{c}\text { Administration } \\
\text { publique }\end{array}$ & $\begin{array}{c}\text { Entreprise } \\
\text { publique }\end{array}$ & $\begin{array}{c}\text { Entreprise } \\
\text { d'un parent }\end{array}$ & $\begin{array}{c}\text { Entreprise privée } \\
\text { tunisienne }\end{array}$ & $\begin{array}{c}\text { Entreprise } \\
\text { mixte }\end{array}$ & $\begin{array}{c}\text { Entreprise } \\
\text { privée étrangère }\end{array}$ & Total \\
\hline 67,7 & 24,9 & 2,5 & 2,5 & 1,5 & 1 & 100 \\
\hline
\end{tabular}

Source: Enquête S. Ben Sedrine \& E. Gobe, 2000.

En raison du développement des établissements tunisiens délivrant un diplôme d'ingénieur (agronome ou autre), on note une régression de la place des cadres techniques formés à l'étranger et notamment en France. 


\section{La montée en puissance des ingénieurs formés en Tunisie : un produit de la tunisification de l'enseignement supérieur}

Quelle place est accordée aux ingénieurs formés à l'étranger, plus particulièrement dans I'ancienne puissance coloniale ? Elle est très importante. Entre 1960 et 1969, plus de la moitié (59\%) des ingénieurs tunisiens inscrits au tableau del'Ordre des ingénieurs ont obtenu un diplôme français. Ils représentent encore le premier contingent des diplômés au début des années 1970 (46,7\% pour 1970 et 1974) $)^{44}$. Au fur et à mesure de la mise en place d'établissements supérieurs techniques, le nombre d'ingénieurs tunisiens formés en France tend à diminuer si on le rapporte à l'ensemble des titulaires d'un diplôme d'ingénieur. Ils constituent respectivement $28,2 \%$ des ingénieurs de l'échantillon qui ont obtenu leur diplôme avant 1980, 16,3\% de ceux qui sont sortis d'une école entre 1981 et 1985, et seulement $11,9 \%$ des diplômés durant la période 1986-1990. Sur les dix dernières années, la proportion de cadres techniques tunisiens formés en France et retournant en Tunisiese maintient autour de $8 \%$, ce qui représente, tant en valeur absolue que relative, le premier contingent des ingénieurs ayant suivi leur cursus à l'étranger (cf. tableau 5).

De leur côté, les effectifs formés en Tunisie rapportés aux titulaires d'un diplôme d'ingénieur se sont fortement accrus. A partir du moment ou les pouvoirs publics ont mis en place une filière tunisienne d'ingénierie hors agriculture, ces derniers forment la majorité des diplômés. Leur effectif passe de 59,3 \% (1981-1985) à 74,4\% (1991-1995) et 85,7 \% (après 1995) de l'ensemble des ingénieurs diplômés (cf. tableau 5). Cette tendance se traduit par une augmentation du nombre des diplômes d'ingénieurs délivrés par les établissements habilités. Le flux annuel des ingénieurs issus des écoles tunisiennes est passé de 676 à 775 entre 1989 et 1994 pour atteindre le chiffre de 1021 en 2000"45. Cette croissance pose la question de la massification du système formation des ingénieurs. Si le nombre de diplômés progresse chaque année, les capacités d'accueil en termes de locaux et d'enseignants des écoles d'ingénieurs restent les mêmes depuis le milieu de la décennie ${ }^{46}$. De manière générale, l'effort financier de l'Etat n'a pas suivi la croissance de l'effectif étudiant. Les coûts unitaires de fonctionnement de l'enseignement supérieur en dinar de 1990 sont passés de 2462 dinars en 1981 à 1390 dinars en 199477. 
Tableau 5. Distribution des flux d'ingénieurs par pays de formation selon la période d'obtention du diplôme (en \%)

\begin{tabular}{|l|c|c|c|c|c|}
\hline Pays de formation & $\mathbf{1 9 8 0} \&$ avant & $\mathbf{1 9 8 1 - 1 9 8 5}$ & $\mathbf{1 9 8 6 - 1 9 9 0}$ & $\mathbf{1 9 9 1 - 1 9 9 5}$ & A près 1995 \\
\hline Tunisie & 45,6 & 59,3 & 65 & 74,4 & 85,7 \\
\hline Pays arabes & 13,6 & 9,9 & 5,1 & 7,9 & 2,5 \\
\hline France & 28,2 & 16,3 & 11,9 & 7,9 & 8 \\
\hline Europe de I'Ouest & 6,8 & 2,9 & 7,9 & 4,9 & 0,8 \\
\hline Europe de I'Est & 2,9 & 8,1 & 5,1 & 3,7 & 0,8 \\
\hline Amérique du Nord & 2,9 & 2,9 & 5,1 & 1,2 & \\
\hline Autre & 0,6 & & & & 1,7 \\
\hline
\end{tabular}

Source: Enquête S. Ben Sedrine \& E. Gobe, 2000.

La distribution des ingénieurs par pays de formation selon la période d'obtention du diplôme peut s'expliquer tout d'abord par la politique des Etats européens qui ont progressivement fermé leurs frontières aux étudiants maghrébins. Elle s'explique aussi par celle des autorités qui, désormais, dissuadent les étudiants tunisiens de suivre à l'étranger des cursus existant déjà dans le pays d'origine, notamment en accordant de moins en moins de bourses aux étudiants des deux premiers cycles ${ }^{48}$. N éanmoins, bien que la France, par exemple, se soit progressivement fermée aux étudiants des premier et deuxième cycles, elle reste l'une des destinations d'excellence universitaire. Pour l'année académique 1996-1997, on a compté quatre-vingt-un lauréats tunisiens des classes préparatoires, dont huit admis à l'Ecole polytechnique et quatorze à l'Ecole centrale de Paris. En 1998-1999, 91 étudiants tunisiens ont réussi le concours des écoles françaises d'ingénieurs ${ }^{49}$. La structure de répartition des diplômés en fonction du grade montre que l'élite technique tunisienne a été formée principalement dans des grandes écoles françaises et marginalement dans les universités nord-américaines les plus prestigieuses.

\section{LE MODÈLE DE FORMATION DES INGÉNIEURS TUNISIENS: UN SYSTĖME DUALISTE}

\section{« La fuite des cerveaux » : résultat de l'existence de filières d'excellence étrangères ?}

La structure de qualification des ingénieurs tunisiens formés à l'étranger montre le caractère d'excellence de certaines destinations. Certains pays forment plus que d'autres des « ingénieurs de conception » portant le titre d'ingénieurs principaux. Près de $95 \%$ des ingénieurs formés en Amérique du N ord (Etats-Unis et Canada) sont des « ingénieurs principaux ». Les « ingénieurs techniciens » se retrouvent en majorité parmi les sortants 
des établissements moins prestigieux comme les établissements tunisiens, les écoles d'autres pays arabes ou celles des pays de l'Est (cf. graphique2) ${ }^{50}$. En revancheen France, les deux groupes se répartissent à peu près à égalité entre « ingénieurs techniciens » et « ingénieurs principaux », le grade d'ingénieur principal étant attribué principalement aux diplômés des grandes écoles.

\section{G raphique 2 : Structure par grade des ingénieurs selon le pays de formation}

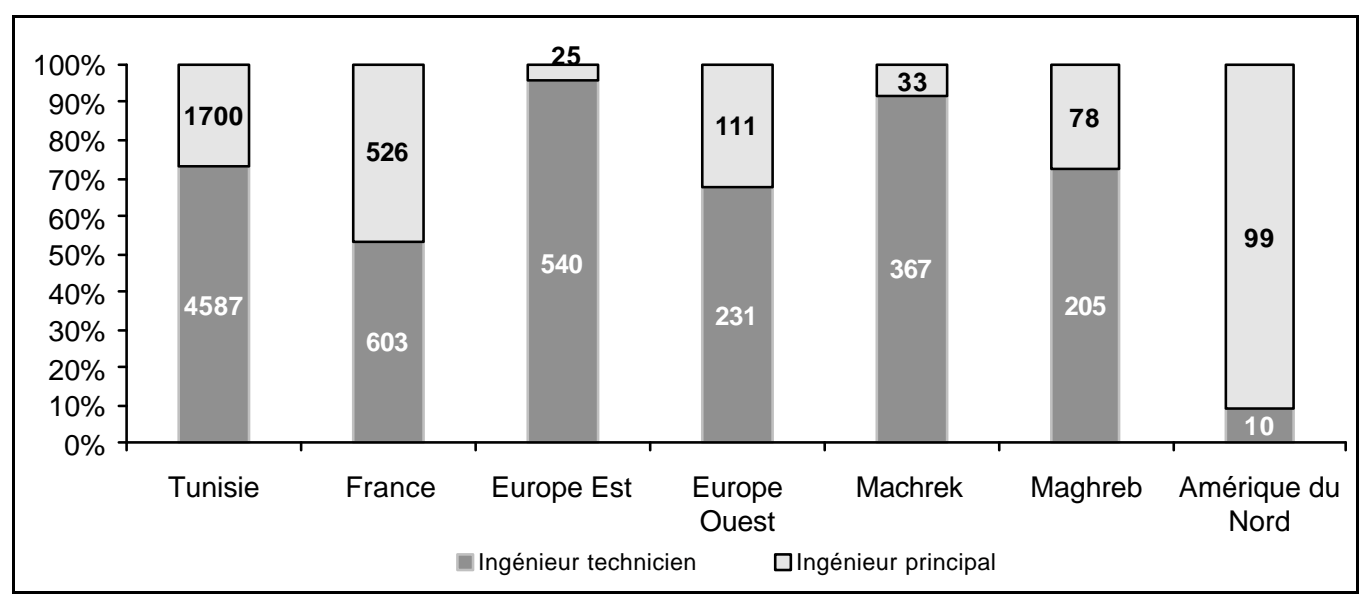

Source: Base de données de l'Ordre des ingénieurs (1999), exploitation statistique par S. Ben Sedrine \& E. Gobe

Ces données concernent, bien sûr, les titulaires d'un diplôme d'ingénieur qui sont rentrés en Tunisie. La coopération universitaire internationale, notamment avec I'ancienne puissance « protectrice », a certainement contribué à améliorer la qualité de la formation des cadres techniques. Elle a également permis de former des compétences de haut niveau et dans des spécialités adaptées aux besoins de l'économie du pays ${ }^{51}$. Cependant, certains ingénieurs ayant occupé de hautes fonctions déplorent l'hémorragie de compétences scientifiques et techniques que cette migration des élèves ingénieurs aurait entraîné et dont la Tunisie serait la victime ${ }^{52}$. II est pourtant difficile de mesurer ce brain-drain, en raison de l'absence d'outils statistiques donnant la possibilité d'évaluer précisément les flux de départ et de retour des élèves ingénieurs. Certaines données partielles peuvent apporter un éclairage sur l'ampleur du phénomène. L'exploitation de I'annuaire de l'Association des Tunisiens des grandes écoles (ATUGE) ${ }^{53}$ fournit des indices sur le retour ou lenon-retour des ingénieurs tunisiens formés en France. En outre, ce type de document, en donnant la situation professionnelle des diplômés répertoriés par I'ATUGE à une date donnée, ne permet pas de tracer leur trajectoire professionnelle. 
II n'autorise pas de réponse à un questionnement sur le retour éventuel des ingénieurs qui ont fait en partie leur carrière en France ou ailleurs. Les réflexions des neuf lauréats tunisiens de la promotion 2000 de l'Ecole polytechnique semblent confirmer cette remarque. Interrogés par un journaliste du magazine tunisien Réalités, ces derniers affirment en chœur que rien ne vaut une expérience professionnelle dans le pays d'accueil et que, par conséquent, ils comptent intégrer des entreprises françaises à la fin de leurs études, c'est-à-dire après avoir suivi une formation complémentaire dans une école d'application (les Mines, les Ponts et Chaussées ou encore Télécom Paris) ${ }^{54}$.

L'annuaire de l'ATUGE n'est donc pas exhaustif et ne précise pas toujours le pays d'emploi des personnes citées ${ }^{55}$. N ous avons pu établir le lieu de travail de 288 ingénieurs ayant obtenu leur titre entre 1990 et 1999 (sur un total de 532). II convient également de noter que quatorze d'entre eux, plus particulièrement parmi les diplômés les plus récents, poursuivent une thèse de doctorat ${ }^{56}$. Le retour en Tunisie ne concernerait que $24,6 \%$ des individus que nous avons repérés. Les diplômés des grandes écoles les plus prestigieuses s'établissent dans leur quasi-total ité dans le pays de formation : 35 des 37 polytechniciens, dont nous avons déterminé le lieu de travail, occupent un emploi en France, 1 en Tunisie et le dernier en Amérique du Nord-Europe. Ces données peuvent être considérées comme fiables dans la mesure où dans 11 cas seulement nous n'avons pas pu identifier le pays d'emploi des polytechniciens. En ce qui concerne les élèves issus des Ponts et Chaussés, 8 sur 10 s'insèrent en France et en Amérique du Nord ou Europe (sur un total répertorié de 14). Quant aux élèves de l'Ecole centrale de Paris 57 , ils retournent peu en Tunisie (3 sur 34) et 27 d'entre eux occupent un emploi dans la capitale française et sa banlieue (comme l'écrasante majorité des diplômés d'une école située à Paris ou dans sa région). A I'opposé, des écoles moins prestigieuses (mais une minorité quand même) voient leurs élèves retourner en Tunisie dans de plus fortes proportions. Par exemple, les deux-tiers des étudiants de l'ESTP ont regagné la Tunisie (10 sur 15) : ce chiffre nous paraît significatif dans la mesure où nous n'avons pas pu déterminer le pays d'emploi de seulement 8 ingénieurs issus de cette école.

Dans le cas de I'ENSAM, il est plus difficile de tirer des conclusions. Cet établissement est, avec l'Ecole centrale de Paris, celui qui a formé le plus gros des contingents des adhérents de l'ATUGE. Sur les 70 «gadzarts » tunisiens sortis entre 1990 et 1999, 25 ont un emploi à l'étranger et 10 exercent en Tunisie ${ }^{58}$. 
Tableau 6. Pays d'emploi des ingénieurs répertoriés par l'A ssociation des Tunisiens des grandes écoles (ATU G E) (1990-1999)

\begin{tabular}{|c|c|c|c|c|}
\hline $\begin{array}{l}\text { Pays d'emploi } \\
\text { inconnu }\end{array}$ & $\begin{array}{c}7 \\
4 \\
27 \\
\end{array}$ & $\begin{array}{l}2 \\
7 \\
3 \\
\end{array}$ & $\begin{array}{l}\text { A mérique du } \\
\text { Nord-Europe }\end{array}$ & $\begin{array}{c}\text { Ecoles } \\
\text { de formation } \\
\text { EC Lille } \\
\text { EC Lyon } \\
\text { EC Paris }\end{array}$ \\
\hline & 3 & 1 & 1 & ENAC \\
\hline & $\begin{array}{c}7 \\
20 \\
\end{array}$ & $\begin{array}{c}2 \\
13 \\
\end{array}$ & $\begin{array}{l}1 \\
2 \\
\end{array}$ & $\begin{array}{c}\text { ENPC } \\
\text { ENSAM } \\
\end{array}$ \\
\hline & 3 & & & ENS UIm \\
\hline & 5 & 3 & 1 & ENSAE Paris \\
\hline & $\begin{array}{l}1 \\
1 \\
\end{array}$ & 2 & & $\begin{array}{c}\text { ENSAI } \\
\text { ENSAT } \\
\text { ENSBANA } \\
\end{array}$ \\
\hline & 2 & & & ENSE A \\
\hline & 1 & 1 & & ENSEEG \\
\hline & 6 & $\begin{array}{l}3 \\
4 \\
\end{array}$ & & $\begin{array}{c}\text { ENSEM } \\
\text { ENSEEIHT }\end{array}$ \\
\hline & 2 & 1 & & EN SER (Grenoble) \\
\hline & $\begin{array}{l}1 \\
2 \\
2 \\
\end{array}$ & $\begin{array}{l}1 \\
1 \\
\end{array}$ & & $\begin{array}{c}\text { ENSHMG } \\
\text { ENSI (Caen) } \\
\text { ENSIEG } \\
\end{array}$ \\
\hline & 3 & & & ENSIA \\
\hline & 1 & & & ESIM \\
\hline & 10 & $\begin{array}{l}2 \\
4 \\
1 \\
\end{array}$ & & $\begin{array}{c}\text { ENSIMAG } \\
\text { ENSITM } \\
\text { ENSMM } \\
\end{array}$ \\
\hline & 1 & & & ENSP (Grenoble) \\
\hline & $\begin{array}{l}1 \\
5 \\
5\end{array}$ & $\begin{array}{c}1 \\
10 \\
\end{array}$ & & $\begin{array}{c}\text { ENSP (Marseille) } \\
\text { ENSTA } \\
\text { ESTP }\end{array}$ \\
\hline & 4 & & & IIE \\
\hline & $\begin{array}{l}4 \\
4 \\
1 \\
\end{array}$ & 2 & & $\begin{array}{c}\text { INAPG } \\
\text { INSA (Toulouse) } \\
\text { INSA (Rouen) } \\
\end{array}$ \\
\hline & 3 & 2 & 1 & INSA (Lyon) \\
\hline & 9 & 1 & & INT \\
\hline & $\begin{array}{l}2 \\
3 \\
\end{array}$ & & 1 & $\begin{array}{c}\text { Mines de Nancy } \\
\text { Mines de Paris }\end{array}$ \\
\hline & 1 & & & Mines de St.-Etienne \\
\hline & 1 & & & SUPAERO \\
\hline & $\begin{array}{c}7 \\
1 \\
10 \\
\end{array}$ & $\begin{array}{l}1 \\
2 \\
\end{array}$ & & $\begin{array}{c}\text { SUPELEC } \\
\text { TELECOM Bretagne } \\
\text { TELECOM Paris } \\
\end{array}$ \\
\hline & 1 & & & $\mathrm{X}$ \\
\hline & $\begin{array}{l}6 \\
9 \\
5 \\
\end{array}$ & & & $\begin{array}{c}X-\text { ENPC } \\
X-\text { ENSAE } \\
X-\text { ENSTA }\end{array}$ \\
\hline & 1 & & & $\mathrm{X}$ - Mines de Paris \\
\hline Total : 244 & $\begin{array}{c}3 \\
10 \\
\text { Total : } 205\end{array}$ & $\begin{array}{c}1 \\
\text { Total : } 71\end{array}$ & $\begin{array}{c}1 \\
\text { Total : } 12\end{array}$ & $\begin{array}{l}X \text { - SUPELEC } \\
\text { X - TELECOM }\end{array}$ \\
\hline
\end{tabular}


Les données recueillies pour les années 1980 sont mal heureusement très lacunaires, le nombre de diplômés dont nous n'avons pas pu déterminer le lieu de travail étant particulièrement élevé (120 des 247 ingénieurs comptabilisés). Sur les 127 personnes dont le pays d'emploi est connu, 68 travaillent en Tunisie, 54 en France, 3 aux Etats-Unis, 1 en Allemagne et enfin 1 au Maroc (employé par une société française) ${ }^{59}$.

Les données sont complètes à une exception près pour les polytechniciens : il existe uneégal ité numérique parfaite entre polytechniciens occupant un emploi en Tunisie et en France $(11)^{60}$. De son côté, I'EN SAM a formé 73 ingénieurs tunisiens pendant la décennie 1980, mais le lieu de travail n'a pu être identifié que pour 23 « gadzarts » (8 occupent un emploi en Tunisie, 14 en France et 1 au Maroc).

Le contraste est patent par rapport aux années 1970 : durant la décennie, plus de $90 \%$ des individus dont nous avons déterminé le lieu de travail sont retournés en Tunisie. Sur un total de 126 ingénieurs ayant obtenu leur diplôme entre 1970 et 1979, 6 exercent un emploi en France, 1 dans les Emirats arabes unis (pour le compte d'une grande entreprise française) et 68 en Tunisie. Nous n'avons cependant pas pu avoir connaissance du lieu de travail de 51 personnes.

L'exploitation de l'annuaire de I'ATUGE permet d'affirmer sans risques que l'émigration des compétences scientifiques et techniques vers le pays de formation, quasi absente dans la décennie 1970, a progressé dans les années 1980, avant de s'accélérer considérablement dans la décennie 1990. Pourtant la récession et la croissance faible qui ont sévi en Franceentre 1992 et 1997, ainsi queles difficultés de séjour découlant d'une politique de contrôle de l'immigration très stricte, auront probablement incité certains jeunes ingénieurs à retourner au pays ${ }^{61}$. Cette migration des compétences aurait été certainement beaucoup plus forte si la France avait connu des taux de croissance plus élevés.

Le Technology Transfer Program (TTP), mis en œuvre d'un commun accord par les Etats-Unis et la Tunisie entre 1981 et 1990, illustre également cette « fuite des cerveaux ». Programme de coopération financé par la U nited States Agency for International D evelopement (USAID), le TTP se proposait de recruter chaque année 250 étudiants, dont 200 bacheliers choisis parmi les plus brillants, distingués au moins par une mention « bien »; les cinquante autres étant destinés à suivre des troisièmes cycles. Un peu plus de la moitié des étudiants étaient orientés vers le génie civil, électrique et mécanique, tandis que les $48 \%$ restants se répartissaient entre les autres disciplines scientifiques ${ }^{62}$. En 1990, les bénéficiaires de ces bourses résidant sur le territoire américain, les 
responsables du TTP ont décidé de développer un projet de suivi (follow up) pour les inciter à rentrer en Tunisie et à s'insérer dans le secteur privé. Doté d'un budget annuel de 100000 \$, ce programmen'a pas produit les effets escomptés : en 1993, seulement 44\% des 763 étudiants ayant bénéficié de ce programme américain étaient retournés au pays. Ce taux s'est un peu amélioré l'année suivante et à la clôture du programme en septembre 1994, il atteignait le chiffre de $56 \% 63$.

C'est en partie pour limiter autant que faire se peut les migrations de compétences scientifiques et techniques qu'une réforme du cursus d'ingénieur a été mise en œuvre à partir de 1989. Elle visait également à gommer toute distinction entre ingénieur technicien et ingénieur principal.

\section{La réforme du cursus d'ingénieur : prégnance du modèle français et institutionalisation d'un double dualisme}

Vingt ans après la création de l'ENIT, certains enseignants et quelques ingénieurs commencent à formuler un certain nombre de critiques à l'égard du système de formation. Deux commissions ministérielles sont alors chargées d'examiner, respectivement, la situation des cycles préparatoires aux études d'ingénieurs et celle de l'enseignement supérieur technique ${ }^{64}$.

Les critiques formulées au sein de ces commissions peuvent être regroupées en deux catégories. La première, et la principale, met en évidence les faibles rendements des écoles d'ingénieurs. Sur l'ensemble des bacheliers orientés vers les études d'ingénieurs, seulement $10 \%$ sortaient avec un diplôme d'ingénieur principal et $30 \%$ avec celui d'ingénieur technicien ${ }^{65}$. Ces résultats sont en outre mal vécus par les élèves ingénieurs de la filière moyenne qui ne peuvent entrer dans la fonction publique qu'avec le grade d'ingénieur des travaux de l'Etat. Leurs perspectives de carrière sont ainsi limitées dans la mesure où ils sont obligés de passer par la formation continue ou par des concours pour accéder au grade d'ingénieur principal. De fait, dans leur majorité, les élèves de la filière bac +6 redoublent après une année de préparation au sein de la filière bac +4 et la majorité de ceux qui sont recrutés pour devenir des ingénieurs techniciens se trouvent relégués dans la filière de techniciens supérieurs. Ces arguments sont explicites dans les propos du rapporteur de la commission de réforme du cycle préparatoire: 


\begin{abstract}
«Les statistiques montrent que plus de $50 \%$ des étudiants de bac +4 étaient des anciens de la filière bac +6 . Et les bac +2 étai ent des ex bac +4 ! O n retrouvait le même phénomène dans toutes les écoles qui d'ailleurs avaient été calquées sur le modèle de I'EN IT. Pourquoi un tel déchet ? Les élèves arrivaient en "prépa" avec d'excellentes moyennes au baccalauréat, mais en fait ils avaient été dopés grâce à des cours particuliers de mathématique et de physique. Ils n'avaient pas l'étoffe pour suivre la filière longue et se retrouvaient en bac +4 . Ils pensaient rentrer par la grande porte et passaient par le trou de la serrure ! L'ingénieur de production, celui dont on a besoin, était ravalé au rang d'un ingénieur de seconde zone qui était recruté dans l'administration avec le grade d'ingénieur des travaux de l'Etat. Les clients de la filière bac +4 étaient pour beaucoup des titulaires du bac technique. On les boxait avec des mathématiques et de la physique. IIs tombaient $\mathrm{KO}$, on oubliait leurs qualités de techniciens et on les éjectait vers la filière moyenne. »66
\end{abstract}

L'âge tardif d'obtention du diplôme parmi les ingénieurs inscrits au tableau de l'Ordre des ingénieurs vient corroborer cette affirmation. Le graphique 3 montre que le diplôme d'ingénieur a été obtenu en Tunisie souvent à un âge supérieur à l'âge théorique, donc à la suite d'un ou plusieurs redoublement(s) au cours du cursus scolaire. Or, si l'étudiant n'effectue aucun redoublement durant tout le cursus scolaire, c'est-à-dire du primaire au supérieur, l'âge théorique d'obtention du diplôme est de 22 ans pour l'ingénieur technicien $(\mathrm{Bac}+4)$ et de 24 ans pour l'ingénieur principal $(\mathrm{Bac}+6)$. Au sommet de la courbe, on voit que l'effectif correspond aux individus ayant effectué seulement un redoublement entre la base et le sommet de la pyramide du système éducatif, tandis que l'effectif, à droite de ce sommet, représente les ingénieurs dont la trajectoire scolaire est marquée par plus d'un redoublement. Enfin à gauche du sommet de la courbe, se trouve la rare catégorie d'ingénieurs qui a obtenu son diplôme en avance, soit à un âge inférieur à l'âge théorique (cf. graphique 3, p. 59).

La deuxième critique formulée par la commission ministérielle concerne l'existence d'un cycle préparatoire intégré au sein des établissements. Avec l'accroissement du nombre d'étudiants accédant à l'enseignement supérieur, les écoles d'ingénieurs, en premier lieu I'ENIT, doivent gérer des collèges préparatoires hypertrophiés :

\footnotetext{
«A vec l'augmentation des effectifs de bacheliers, on nous demandait de prendre de plus en plus d'élèves en "prépa" (... ). L'EN IT est ainsi devenu un monstre avec une grosse tête: la préparation, et des petites jambes : les deux filières d'ingénieurs. II y avait, dans les années 1980, 1600 étudiants à I'EN IT, la filière moyenne et la filière longue ne dépassaient pas 200 élèves (... ). L'ENIT est devenu une école de préparation et de filière courte et accessoirement une formation d'ingénieur. »
} 


\section{G raphique 3 : D istribution par grade selon l'âge d'obtention du diplôme Ingénieurs formés en Tunisie}

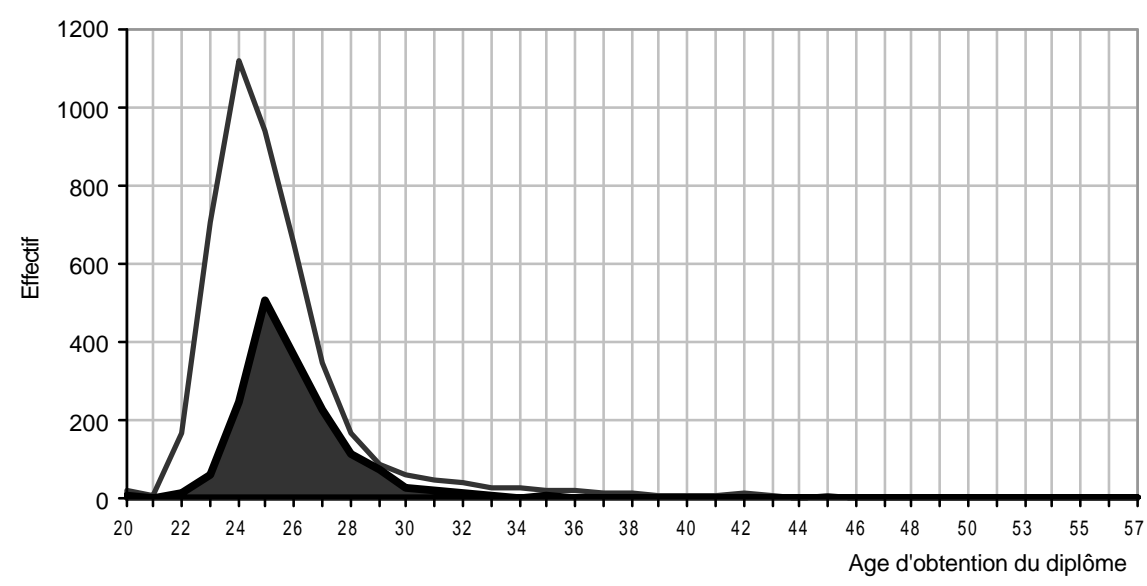

口Ing Technicien

OIng Principal

\begin{tabular}{|c|c|c|c|c|c|c|c|c|c|c|c|c|c|c|c|c|c|c|c|}
\hline \multirow{2}{*}{$\begin{array}{c}\text { Cycle } \\
\text { Niveau }\end{array}$} & \multicolumn{9}{|c|}{ Enseignement de base } & \multicolumn{4}{|c|}{$\begin{array}{l}\text { Enseignement } \\
\text { secondaire }\end{array}$} & \multicolumn{6}{|c|}{ Enseignement supérieur } \\
\hline & 1 & 2 & 3 & 4 & 5 & 6 & 7 & 8 & 9 & 1 & 2 & 3 & BAC & 1 & 2 & 3 & 4 & 5 & 6 \\
\hline Age & 6 & 7 & 8 & 9 & 10 & 11 & 12 & 13 & 14 & 15 & 16 & 17 & 18 & 19 & 20 & 21 & 22 & 23 & 24 \\
\hline théorique & ans & ans & ans & ans & ans & ans & ans & ans & ans & ans & ans & ans & ans & ans & ans & ans & ans & ans & ans \\
\hline
\end{tabular}

Ingénieur technicien $=\mathrm{Bac}+4$ (âge théorique $=22$ ans )

Ingénieur principal $=\mathrm{Bac}+6$ (âge théorique $=24$ ans $)$

Source : Base de données de l'Ordre des ingénieurs (1999), exploitation par S. Ben Sedrine \& E. Gobe

Autre facteur déterminant, le souvenir de l'agitation estudiantine islamiste dans les écoles d'ingénieurs, pendant la première moitié des années 1980, a contribué à faire avancer l'idée que des cycles préparatoires indépendants des écoles d'ingénieurs pourraient préserver le calme dans les établissements :

«A voir des cycles préparatoires intégrés dans les écoles n'étaient pas sans inconvénients. Les universités étaient perturbées à cette époque. Or, si la moitié du programmen'était pas étudié en raison des grèves, les enseignants ne posaient pas de sujets dessus. D u coup, la formation était tronquée. Tandis qu'avec un système préparatoire indépendant du cycle ingénieur, les élèves courent derrière leur professeur pour terminer le programme, tout simplement parce que ceux qui posent les sujets des concours nationaux d'entrée aux différentes écoles d'ingénieurs ne sont pas ceux qui enseignent dans le cycle préparatoire. »68

Un cycle préparatoire séparé est également censé introduire la concurrence entre des élèves qui désormais tenteraient d'intégrer la meilleure école ${ }^{69}$. De plus, la coexistence de filières courte, moyenne et longue dans le même établissement aurait été préjudiciable au contenu des enseignements. II apparaît, en effet, que ce système conduit à une redondance des programmes pour les nombreux redoublants qui passent dans les filières «ingénieurs de production » et «techniciens supérieurs ». Les enseignants qui souvent dispensent des cours dans les différents niveaux sont, selon Taïeb Hadhri, «amenés à enseigner des recettes aux ingénieurs et trop de théorie aux techniciens ${ }^{70}$. Un autre 
argument est également avancé pour dénoncer les limites du double cursus : l'âge moyen de l'ingénieur principal serait supérieur au standard international et découragerait ceux qui envisagent de poursuivre des études doctorales.

La réforme qui allait ainsi mettre fin à la dualité de la formation ingénieur technicien-ingénieur principal prévoit d'homogénéiser à cinq ans l'ensemble des formations. En ce sens, elle s'inspire du modèle des «écoles traditionnelles françaises » qui recrutent leurs élèves après deux années de classe préparatoire scientifique et qui proposent un cursus de trois ans. A près deux années préparatoires dans un institut, les étudiants tunisiens passent un concours national sur épreuves écrites et orales qui leur donne accès aux écoles ou aux départements des facultés des sciences formant des ingénieurs. Ces établissements délivrent désormais un diplôme unique appelé «diplôme national d'ingénieur » (DNI). Aussi n'existe-t-il plus de critères juridiques différenciant « ingénieur de production » et «ingénieur de conception », les filières et les options choisies par les étudiants au sein des écoles devant déterminer les caractéristiques professionnelles des cadres techniques.

Ce modèle est défendu par le principal promoteur de la réforme avec d'autant plus de vigueur que le Maroc a ouvert en 1985 et en 1986 une série de «math sup. » et de « math spé. » dans plusieurs lycées de Rabat, Casablanca et Fès ${ }^{71}$. Ce projet marocain a été financé et soutenu par la coopération française qui a mis à la disposition des classes préparatoires la quasi-totalité des enseignants; et a soutenu la création par les autorités marocaines de centres de préparation aux agrégations scientifiques, afin de remplacer à moyen terme les coopérants français ${ }^{72}$.

A partir des recommandations présentées par la commission et avec le soutien de la coopération française, le ministère tunisien de l'Education et des Sciences a élaboré alors le schéma du système préparatoire actuellement en vigueur. Un nouvel établissement de préparation, I'Institut préparatoire aux études scientifiques et techniques (IPEST) voit le jour en juin 1991. Dans un premier temps, l'A mbassade de France met à la disposition de l'établissement la majeure partie du corps enseignant : celui-ci doit assurer la préparation de professeurs tunisiens aux agrégations de physique et de mathématiques, afin de former en priorité des agrégés qui prendront à l'avenir la place des coopérants. Cette préparation à l'agrégation a également pour but de revaloriser le statut du corps des enseignants agrégés et d'éviter qu'ils ne soient tentés d'obtenir un doctorat, seul diplôme donnant la possibilité de développer une carrière au sein del'université tunisienne ${ }^{73}$. 
Les promoteurs du projet tendent à cal quer l'agrégation tunisienne sur l'agrégation française. Pour ce faire, les agrégatifs tunisiens doivent traiter les mêmes sujets qu'en France $^{74}$. Une fois l'admissibilité prononcée, les candidats passent les oraux devant un jury mixte de professeurs tunisiens et français. Quant au cycle préparatoire, il est mis en place à la rentrée de 1992. Les élèves ne sont plus sélectionnés uniquement en fonction du score au baccalauréat (voir infra), mais également à partir du dossier scolaire, plus précisément, à partir des résultats obtenus dans les trois dernières années du secondaire. II s'agit de ne pas écarter d'un cursus d'excellence les étudiants ayant eu des résultats moyens au baccalauréat ${ }^{75}$. Les 115 bacheliers inscrits à I'IPEST ${ }^{76}$ passent les concours d'entrée aux écoles françaises «traditionnelles » d'ingénieurs pour lesquels des centres d'écrit sont ouverts à Tunis. Mais ils présentent aussi leur candidature pour les concours nationaux d'accès aux cycles de formation d'ingénieur. Afin de garder d'excellents étudiants en Tunisie et améliorer le niveau des écoles tunisiennes, il a été prévu, dans un premier temps, que les bourses ne seraient allouées qu'aux lauréats des établissements français les plus prestigieux (Polytechnique, Ponts et Chaussées, Centrale, les Mines, Télécom) $)^{77}$.

Outre l'IPEST, des Instituts préparatoires aux études d'ingénieurs (IPEI) sont créés sur l'ensemble du territoire national. Mais ces derniers sont entièrement dédiés à la préparation du concours donnant accès aux écoles ou aux départements des facultés des sciences délivrant des diplômes d'ingénieur. A la différence de ceux de I'IPEST, les étudiants intégrant ces IPEI sont recrutés seulement sur la base du score au baccalauréat ${ }^{78}$. Ces préparations ont commencé à fonctionner en 1992, la première session du concours national s'est déroulée en juin 1994, et les premiers DNI ont été délivrés en juillet $1997^{79}$.

Pour autant, le nouveau système de formation ne résout pas la question de la dualité du système d'enseignement supérieur. En effet, d'une année sur l'autre, les 20 à 30 premiers du baccalauréat optent pour une scolarité dans les classes préparatoires françaises, les 30 suivants partent en Allemagne, tandis que plus d'une centaine d'étudiants s'inscrivent à I'IPEST. II convient de signaler que le nombre d'étudiants de I'IPEST réussissant chaque année à intégrer une école française d'ingénieurs n'a cessé de progresser (de 9 en 1994 à 78 en 2001).

Le premier directeur de I'IPEST, Mohamed Jaoua ${ }^{80}$, a tenté de résoudre la quadrature du cercle en proposant la création d'une école polytechnique à Tunis, destinée à recruter les étudiants de qualité ne pouvant se rendre à l'étranger faute de 
places. A l'origine de cette décision, on trouve, là encore, le constat que les ingénieurs formés dans les grandes écoles françaises, plus particulièrement ceux de Polytechnique et de I'Ecole centrale de Paris ne rentrent plus en Tunisie. Par conséquent, I'Ecole polytechnique de Tunis (EPT) a été conçue comme devant former des «ingénieurs de conception » du même profil que ceux issus des deux grandes écoles françaises 81 .

Par ailleurs, le promoteur du projet souhaitait égal ement voir les bacheliers les plus performants suivre un cursus d'ingénieur plutôt que s'orienter vers des études de médecine ou de gestion. Taïeb Hadhri fait remarquer à ce sujet:

\footnotetext{
«D e jeunes bacheliers brillants ont commencé à être attirés de plus en plus vers la médecine ou la gestion. II était nécessaire de trouver le bon équilibre. L'EPT est un modèle qui est là pour tirer vers le haut l'ensemble des formations d'ingénieur et motiver les jeunes à suivre des car rières techniques de haut niveau. »
}

C'est donc dans le contexte de la réforme générale des formations d'ingénieurs que I'EPT voit lejour en juin 1991, puis accueille en 1994 ses premiers élèves. Levivier naturel de recrutement de l'EPT est bien évidemment I'IPEST. Les trois-quarts des étudiants qui chaque année intègrent l'EPT sont passés par cet institut, le quart restant venant des classes préparatoires techniques et de physique-chimie des IPEI. Tous sont boursiers du gouvernement tunisien. Leur statut et leurs conditions de travail témoignent de la volonté des promoteurs de l'Ecole polytechnique de Tunis d'en faire un pôle d'excellence ${ }^{83}$.

En somme, la réforme du cursus des études d'ingéniorat a débouché sur la création de onze institutions chargées des cycles préparatoires et la mise en place de quatre concours aux écoles d'ingénieurs : respectivement, en mathématiques-physique $(\mathrm{MP})$, physique-chimie (PC), biologie-géologie (BG) et technologie (T). Le tableau 7 répertorie les filières existant dans les différentes classes préparatoires tunisiennes et détaille les effectifs accueillis en 1998 en fonction du baccalauréat obtenu. Chacune des 16 institutions formant des ingénieurs ouvre un certain nombre de places dans chacun des concours. Le tableau 8 donne le nombre de places ouvertes pour la session de juin 1998. 
Tableau 7. Les instituts préparatoires : leurs filières d'étude et les capacités d'accueil offertes(1998)

\begin{tabular}{|c|c|c|c|c|}
\hline \multirow[t]{2}{*}{ Institutions } & \multirow[t]{2}{*}{ Filières } & \multicolumn{3}{|c|}{ N ature du Baccalauréat } \\
\hline & & M athématiques & $\begin{array}{c}\text { sciences } \\
\text { expérimentales }\end{array}$ & Technique \\
\hline IPEST dela Marsa & MP - PC & 135 & & \\
\hline IPEI deTunis & $M P-P C$ & 330 & 20 & \\
\hline IPEI de Nabeul & $M P-P C-T$ & 400 & 35 & 115 \\
\hline IPEI deSfax & $M P-P C-T-B G$ & 270 & 19 & 200 \\
\hline IPEI de Monastir & $M P-P C-T$ & 315 & 50 & 235 \\
\hline IPEI deSousse & MP - PC & 280 & 20 & \\
\hline IPEI de Mateur & $M P-P C$ & 285 & 20 & \\
\hline IPEI deGabès & $M P-P C-T$ & 200 & 100 & 250 \\
\hline INAT & BG & & 80 & \\
\hline ESHE deChott & BG & & 80 & \\
\hline ESA de Mograne & BG & & 120 & \\
\hline
\end{tabular}

Source : http:/ / www.orientation.tn $\mathrm{MP}=$ mathématiques-physique, $\mathrm{PC}=$ physique-chimie, $\mathrm{BG}=$ biologie-géologie, $\mathrm{T}=$ technologie $(T)$

Tableau 8. Places ouvertes aux concours nationaux d'entrée aux écoles d'ingénieurs (session de juin 1998)

\begin{tabular}{|cccc|c|}
\hline Etablissements & BG & Concours & P P & T \\
\hline ENIG & & 80 & 20 & 45 \\
\hline ENIM & 50 & 70 & 20 & 50 \\
\hline ENIS & & 50 & 55 & 65 \\
\hline ENIT & 160 & 25 & 30 \\
\hline $\begin{array}{c}\text { ENSI } \\
\text { EPT }\end{array}$ & 25 & 75 & 10 & 5 \\
$\begin{array}{c}\text { ESA de Mateur } \\
\text { ESA deM ograne } \\
\text { ESA du Kef }\end{array}$ & 35 & 36 & 6 & 3 \\
$\begin{array}{c}\text { ESHE deChott Mariem } \\
\text { ESIA deTunis }\end{array}$ & 25 & & & \\
\hline ESER de Medjez el Bab & 45 & & & \\
\hline ESPT deTunis & & 15 & 5 & \\
\hline $\begin{array}{c}\text { faculté des sciences deTunis } \\
\text { (filière informatique) }\end{array}$ & & 20 & 5 & 15 \\
\hline $\begin{array}{l}\text { faculté des sciences deTunis } \\
\text { (filière chimieet géosciences) } \\
\text { INAT }\end{array}$ & 5 & 55 & 5 & 10 \\
\hline
\end{tabular}

Source : http:/ / www.orientation.tn

$\mathrm{MP}=$ mathématiques-physique, $\mathrm{PC}=$ physique-chimie, $\mathrm{BG}=$ biologie-géologie, $\mathrm{T}=$ technologie (T) 
Tableau 9. Classement de certains établissements d'enseignement supérieur selon le score du dernier orienté en 1997 (par ordre décroissant)

\begin{tabular}{|c|c|c|c|}
\hline Etablissement & Filière & C apacité d'accueil & $\begin{array}{l}\text { Score du dernier } \\
\text { orienté en fonction } \\
\text { du baccalauréat }\end{array}$ \\
\hline $\begin{array}{l}\text { Institut préparatoire aux } \\
\text { études scientifiques et } \\
\text { techniques (IPEST) } \\
\text { faculté de médecine de Tunis }\end{array}$ & $\begin{array}{c}\text { Cyclepréparatoire } \\
\text { scientifique } \\
\text { Médecine }\end{array}$ & $\begin{array}{l}\text { M*: } 125 \\
\text { SE* : } 170\end{array}$ & $\begin{array}{c}\text { Présélection } \\
+ \\
\text { Score } \\
\text { M : } 175,17 \\
\text { SE : } 156,32\end{array}$ \\
\hline $\begin{array}{c}\text { Institut préparatoire aux } \\
\text { études d'ingénieurs (IPEI) de } \\
\text { Tunis } \\
\end{array}$ & $\begin{array}{l}\text { Cyclepréparatoire } \\
\text { scientifique }\end{array}$ & $\begin{array}{l}M: 330 \\
S E: 20\end{array}$ & $\begin{array}{l}\text { M : } 165,52 \\
\text { SE : } 162,83\end{array}$ \\
\hline $\begin{array}{c}\text { faculté de médecine de } \\
\text { Sousse } \\
\text { faculté de médecine de Sfax }\end{array}$ & $\begin{array}{l}\text { Médecine } \\
\text { Médecine }\end{array}$ & $\begin{array}{l}M: 50 \\
\text { SE }: 75 \\
M: 45 \\
\text { SE }: 90 \\
\end{array}$ & $\begin{array}{l}M: 160,36 \\
\text { SE : } 144,98 \\
M: 159,65 \\
\text { SE : } 150,50\end{array}$ \\
\hline $\begin{array}{c}\text { faculté de médecine de } \\
\text { Monastir } \\
\text { Institut des hautes études } \\
\text { commerciales de Carthage } \\
\text { (IHEC) }\end{array}$ & $\begin{array}{c}\text { Médecine } \\
\text { sciences économiques et de } \\
\text { gestion }\end{array}$ & $\begin{array}{l}M: 50 \\
\text { SE :75 } \\
M: 215 \\
S E: 105\end{array}$ & $\begin{array}{l}M: 160,05 \\
\text { SE :146,63 } \\
M: 152,96 \\
\text { SE : } 140,84\end{array}$ \\
\hline $\begin{array}{l}\text { Institut supérieur de gestion } \\
\text { deTunis(ISG) }\end{array}$ & Informatique de gestion & $\begin{array}{l}M: 40 \\
S E: 50\end{array}$ & $\begin{array}{l}M: 151,83 \\
S E: 143,15\end{array}$ \\
\hline $\begin{array}{c}\text { faculté de pharmacie de } \\
\text { Monastir } \\
\text { faculté de médecine dentaire } \\
\text { deMonastir } \\
\end{array}$ & $\begin{array}{c}\text { Pharmacie } \\
\text { médecinedentaire }\end{array}$ & $\begin{array}{l}M: 60 \\
S E: 80 \\
M: 55 \\
S E: 85\end{array}$ & $\begin{array}{l}M: 150,02 \\
\text { SE : } 139,38 \\
M: 149,32 \\
\text { SE }: 137,64 \\
\end{array}$ \\
\hline $\begin{array}{c}\text { Institut préparatoire aux } \\
\text { études d'ingénieurs (IPEI) de } \\
\text { Nabeul }\end{array}$ & $\begin{array}{c}\text { Cyclepréparatoire } \\
\text { scientifiqueou technique }\end{array}$ & $\begin{array}{l}\mathrm{M}: 400 \\
\mathrm{SE}: 35 \\
\mathrm{~T}: 115\end{array}$ & $\begin{array}{c}\mathrm{M}: 146,41 \\
\mathrm{SE}: 149,05 \\
\mathrm{~T}: 157,48\end{array}$ \\
\hline $\begin{array}{c}\text { Institut supérieur de gestion } \\
\text { (ISG) deTunis }\end{array}$ & $\begin{array}{c}\text { sciences économiques et } \\
\text { gestion }\end{array}$ & $\begin{array}{l}\mathrm{M}: 140 \\
\mathrm{SE}: 170\end{array}$ & $\begin{array}{l}\mathrm{M}: 145,91 \\
\mathrm{SE}: 140,66\end{array}$ \\
\hline $\begin{array}{l}\text { Ecole nationale de médecine } \\
\text { vétérinaire deSidi Thabet }\end{array}$ & médecinevétérinaire & $\begin{array}{l}M: 10 \\
S E: 50\end{array}$ & $\begin{array}{l}\mathrm{M}: 142,88 \\
\mathrm{SE}: 128,14\end{array}$ \\
\hline $\begin{array}{c}\text { Ecole supérieure des sciences } \\
\text { et techniques de la santé de } \\
\text { Tunis }\end{array}$ & Anesthésie & $\begin{array}{l}M: 10 \\
S E: 35\end{array}$ & $\begin{array}{l}\mathrm{M}: 137,28 \\
\mathrm{SE}: 130,55\end{array}$ \\
\hline $\begin{array}{l}\text { Ecole supérieure des sciences } \\
\text { et techniques de la santé de } \\
\text { Monastir }\end{array}$ & Anesthésie & $\begin{array}{l}\mathrm{M}: 30 \\
\mathrm{SE}: 5 \\
\mathrm{~T}: 5\end{array}$ & $\begin{array}{l}\mathrm{M}: 134,42 \\
\mathrm{SE}: 125,96 \\
\mathrm{~T}: 102,02\end{array}$ \\
\hline $\begin{array}{c}\text { Ecole supérieure de } \\
\text { commerce (ESC) de Tunis }\end{array}$ & $\begin{array}{c}\text { sciences économiques et de } \\
\text { gestion }\end{array}$ & $\begin{array}{l}\mathrm{M}: 170 \\
\mathrm{SE}: 145\end{array}$ & $\begin{array}{l}M: 133,96 \\
S E: 130,49\end{array}$ \\
\hline $\begin{array}{c}\text { Ecole nationale } \\
\text { d'architecture et d'urbanisme } \\
\text { deTunis } \\
\text { (ENAU) }\end{array}$ & Architecture et urbanisme & $\begin{array}{l}\mathrm{M}: 55 \\
\mathrm{SE}: 50 \\
\mathrm{~T}: 75\end{array}$ & $\begin{array}{c}\mathrm{M}: 131,11 \\
\mathrm{SE}: 123,81 \\
\mathrm{~T}: 121\end{array}$ \\
\hline
\end{tabular}


Suite tableau 9.

\begin{tabular}{|c|c|c|c|}
\hline $\begin{array}{c}\text { Institut préparatoire aux } \\
\text { études d'ingénieurs (IPEI) de } \\
\text { Monastir } \\
\end{array}$ & $\begin{array}{c}\text { Cycle préparatoire } \\
\text { scientifique ou technique }\end{array}$ & $\begin{array}{l}M: 315 \\
S E: 50 \\
T: 235\end{array}$ & $\begin{array}{l}M: 131,02 \\
\text { SE }: 148,33 \\
T: 139,21\end{array}$ \\
\hline $\begin{array}{c}\text { Institut supérieur degestion } \\
\text { (ISG) de Sousse }\end{array}$ & \begin{tabular}{|c|}
$\begin{array}{c}\text { sciences économiques et de } \\
\text { gestion }\end{array}$ \\
\end{tabular} & $\begin{array}{l}\mathrm{M}: 140 \\
\mathrm{SE}: 195\end{array}$ & $\begin{array}{l}M: 129,43 \\
S E: 128,42\end{array}$ \\
\hline $\begin{array}{c}\text { Ecole supérieure des postes } \\
\text { et des télécommunications de } \\
\text { Tunis } \\
\text { Institut supérieur de } \\
\text { comptabilité et } \\
\text { d'administration des } \\
\text { entreprises de Tunis } \\
\text { (ISCAE) }\end{array}$ & $\begin{array}{l}\text { Technicien supérieur en } \\
\text { Télécommunication } \\
\text { sciences économiques et de } \\
\text { gestion }\end{array}$ & $\begin{array}{c}M: 30 \\
S E: 15 \\
T: 25 \\
M: 150 \\
S E: 120\end{array}$ & $\begin{array}{l}\text { M : } 129,20 \\
\text { SE }: 133,83 \\
\text { T : } 129,66 \\
\text { M : } 127,72 \\
\text { SE : } 127,39\end{array}$ \\
\hline $\begin{array}{l}\text { Institut national des sciences } \\
\text { appliquées et de technologie } \\
\text { (INSAT) }\end{array}$ & $\begin{array}{l}\text { Mathématique-physique } \\
\text { informatique (technicien } \\
\text { supérieur en 3ans) }\end{array}$ & $\begin{array}{l}M: 200 \\
S E: 50 \\
T: 105\end{array}$ & $\begin{array}{c}M: 125,65 \\
S E: 140,93 \\
T: 130,25\end{array}$ \\
\hline $\begin{array}{c}\text { Institut préparatoire aux } \\
\text { études d'ingénieurs (IPEI) de } \\
\text { Mateur }\end{array}$ & $\begin{array}{l}\text { Cycle préparatoire } \\
\text { scientifique }\end{array}$ & $\begin{array}{l}M: 285 \\
S E: 20\end{array}$ & $\begin{array}{c}M: 125,4 \\
\text { SE : } 137,38\end{array}$ \\
\hline $\begin{array}{c}\text { Ecole supérieure de } \\
\text { commerce }(E S C) \text { de Sfax }\end{array}$ & $\begin{array}{c}\begin{array}{c}\text { sciences économiques et de } \\
\text { gestion }\end{array} \\
\end{array}$ & $\begin{array}{l}M: 220 \\
S E: 165\end{array}$ & $\begin{array}{l}M: 123,58 \\
S E: 125,48\end{array}$ \\
\hline $\begin{array}{l}\text { Institut national des sciences } \\
\text { appliquées et de technologie }\end{array}$ & \begin{tabular}{|l|} 
Chimie et biologie \\
\end{tabular} & $\begin{array}{c}\mathrm{M}: 40 \\
\mathrm{SE}: 100 \\
\mathrm{~T}: 5\end{array}$ & $\begin{array}{c}\mathrm{M}: 117,53 \\
\mathrm{SE}: 125,05 \\
\mathrm{~T}: 99,39\end{array}$ \\
\hline $\begin{array}{c}\text { Institut préparatoire aux } \\
\text { études d'ingénieurs (IPEI) de } \\
\text { Sfax } \\
\text { Institut préparatoire aux } \\
\text { études d'ingénieurs (IPEI) } \\
\text { de Gabès }\end{array}$ & $\begin{array}{l}\text { - Cyclepréparatoire } \\
\text { scientifique ou technique } \\
\\
\text { - Cyclepréparatoire } \\
\text { spécialité biologie } \\
\text { Cycle préparatoire } \\
\text { scientifique ou technique }\end{array}$ & $\begin{array}{l}M: 270 \\
\text { SE }: 15 \\
T: 200 \\
\text { SE }: 175 \\
M: 270 \\
\text { SE }: 15 \\
T: 200\end{array}$ & $\begin{array}{l}M: 113,90 \\
\text { SE }: 136,25 \\
T: 133,75 \\
\\
\text { SE }: 97,63 \\
M: 108,14 \\
\text { SE }: 114,05 \\
T: 114,67 \\
\end{array}$ \\
\hline $\begin{array}{c}\text { Institut national } \\
\text { agronomique de Tunis }\end{array}$ & $\begin{array}{l}\text { Cycle préparatoire } \\
\text { spécialité biologie }\end{array}$ & SE : 120 & SE :137,62 \\
\hline $\begin{array}{c}\text { Ecolesupérieure } \\
\text { d'agriculture deChott- } \\
\text { Mariem }\end{array}$ & $\begin{array}{l}\text { Cycle préparatoire } \\
\text { spécialité biologie }\end{array}$ & SE : 80 & SE : 120,90 \\
\hline $\begin{array}{c}\text { Ecolesupérieure } \\
\text { d'agriculture de Mograne } \\
\end{array}$ & $\begin{array}{l}\text { Cycle préparatoire } \\
\text { spécialité biologie }\end{array}$ & SE : 80 & SE : 119,33 \\
\hline $\begin{array}{c}\text { Institut préparatoire aux } \\
\text { études d'ingénieurs (IPEI) de } \\
\text { Sousse } \\
\end{array}$ & $\begin{array}{l}\text { Cycle préparatoire } \\
\text { scientifique }\end{array}$ & $\begin{array}{l}M: 280 \\
S E: 20\end{array}$ & Non mentionné \\
\hline
\end{tabular}

Source : Guide l'Orientation universitaire 1998 (à l'exception de l'IPEST dont le chiffre indiqué est celui de 1998), $M$ = Baccalauréat de mathématiques, $S E$ = Baccalauréat de sciences expérimentales, $T$ = Baccalauréat technique. 
Les données fournies par le Guide de l'O rientation universitaire en 1998, publiées par le ministère de l'Enseignement supérieur, permettent, dans une large mesure, de repérer les filières d'excellence del'enseignement tunisien à la fin de la décennie 1990. Le tableau 9 classe les établissements d'enseignement supérieur en fonction du rang obtenu par le dernier admis à l'Orientation universitaire. L'orientation des bacheliers en Tunisie constitue un moyen de savoir quels établissements et quelles disciplines sont perçus comme les plus prestigieux. Dans un contexte marqué par les possibilités d'accueil limitées des écoles d'ingénieurs et des universités, l'une des principales fonctions du baccalauréat est de réguler le nombre des inscrits de l'Enseignement supérieur. II se rapproche ainsi de la logique du concours d'entrée. Le caractère sélectif du système éducatif supérieur se manifeste également après l'examen du baccalauréat, dans la mesure où, depuis 1976, l'affectation des bacheliers dans l'enseignement supérieur est réalisée par un système d'orientation centralisé, aujourd'hui informatisé, combinant la performance scolaire de l'élève (le score) aux capacités d'accueil des diverses institutions del'enseignement supérieur ${ }^{84}$. Autrement dit, ce procédédistribueles bacheliers dans les établissements en fonction de leurs vœux, des notes obtenues au baccalauréat et, dans une moindre mesure, de leurs résultats pendant la 7ème année du cycle secondaire. A ussi peut-on formuler l'hypothèse que l'attrait du métier d'ingénieur vient en second dans les processus d'orientation universitaire vers les écoles d'ingénieurs.

Ce classement montre que l'instauration d'un DNI n'a pas jusqu'à ce jour contribué à revaloriser l'ensemble de la filière de l'ingéniorat. Au contraire, on assiste plutôt à un processus de déclassement de certains instituts préparatoires, et par-là même de certaines écoles d'ingénieurs. Les scores indiqués donnent à penser que certains élèves ont intégré les instituts préparatoires les moins côtés avec des notes très moyennes au baccalauréat dans les disciplines scientifiques ${ }^{85}$. Les IPEI sont, semble-t-il, conçus davantage comme des établissements offrant une capacité d'accueil en augmentation chaque année que comme des classes préparatoires ${ }^{86}$.

Pour élaborer le classement des divers établissements d'enseignement supérieur, et par-là même des disciplines les plus prisées, nous avons tenu compte du seul score au baccalauréat de mathématiques, le plus prestigieux. En dépit des limites que représente I'usage des scores affichés par ce Guide ${ }^{87}$, on peut tirer certains enseignements du tableau 9. L'IPEST et I'IPEI de Tunis sont classés respectivement premier et troisième des établissements accueillant les élèves ayant les meilleurs résultats au baccalauréat (avec une nuance pour I'IPEST). A ces deux exceptions près, les facultés de médecine 
apparaissent comme les filières d'excellence prisées par les étudiants qui restent en Tunisie pour effectuer leur cursus du moins pour les premier et second cycles. Des écoles de commerce comme I'IHEC ou I'ISG de Tunis (option informatique) se positionnent en second rang. Elles sont suivies par la faculté de pharmacie de Monastir, la faculté de médecine dentaire de la même ville et par I'IPEI de N abeul. Ce dernier vient avant I'IPEI de Monastir, lui-même devancé par I'ISG de Tunis (option sciences économiques et de gestion), l'Ecole nationale de médecine vétérinaire de Sidi Thabet, les Ecoles supérieures des sciences et techniques de la santé (Tunis et Monastir - option anesthésie), l'ESC de Tunis, et I'ENAU. Les IPEI de Mateur et deSfax sont quant à eux devancés par des écoles de commerce (I'ISG de Sousse et I'ISCAE de Tunis) et par des établissements délivrant des diplômes de techniciens supérieurs (I'ESPTT et I'INSAT). Quant à I'IPEI de Gabès, il se situe à la dernière place de la liste ${ }^{88}$.

La hiérarchie scolaire de ces cycles de préparation se retrouve au niveau des écoles d'ingénieurs, tout au moins si l'on se fie aux choix de l'école d'ingénieurs effectués par les étudiants des instituts préparatoires lorsqu'ils constituent leur dossier de candidature aux différents concours nationaux d'entrée. De fait, ils classent les écoles et contribuent ainsi à consacrer leur hiérarchie. L'école polytechnique de Tunis se situe au premier rang, suivie, selon les années, par l'ENIT, I'ENSI et I'ESPTT. A ce propos Taïeb Hadhri fait remarquer que «Les premiers recrutés dans ces trois écoles ont des classements très comparables. Les derniers recrutés à I'ENIT sont les plus faibles parce que cet établissement prend environ 200 étudiants al ors I'ENSI en recrute une centaine et I'ESPTT une soixantain $e^{89}$. 》

L'établissement que l'on intègre à l'issue du cycle préparatoire dépend bien plus de sa place dans une hiérarchie calquée sur la valeur scolaire des candidats admis que des affinités des étudiants pour tel ou tel domaine scientifique ou technique. On se trouve devant une double dualité du système de formation des ingénieurs, entre, d'une part, la Tunisie et des pays commela France ou l'Allemagne continuant de former une «élite » qui dans de fortes proportions reste dans le pays de formation ; et, d'autre part en Tunisie, entre des écoles ayant une vocation d'excellence accueillant les « bons étudiants »n'ayant pas intégré une grande école à l'étranger et des établissements situés en province ou spécialisés, fortement concurrencés par les instituts de gestion et les écoles de commerce.

Dans un tel système, les facultés des sciences apparaissent comme des filières de relégation. C'est tout au moins ce qui ressort de l'entretien accordé au politologue François Siino par un professeur de physique de la faculté des sciences de Tunis : 


\begin{abstract}
«En Tunisie, les institutions qui sont responsables de la formation, ce sont les facultés. M ais si vous regardez bien la réalité tunisienne, les étudiants que l'on envoie dans les facultés, ce sont les étudiants qui n'ont pas trouvé ailleurs où aller. Parce que les meilleurs se détachent par couches successives. Les meilleurs des meilleurs, une cinquantaine, vont en France, au Canada... disons une centaine. Ensuite les meilleurs vont en médecine. Par la suite il y a toutes les préparations qui écrèment, I'IPEST, HEC, I'EN IT, toutes ces institutions-là. Et on crée encore des préparations ; avant il y en avait deux, maintenant, il y en a cinq ou six. Tout ça passe avant. Celui qui peut accéder à ces choses-là ne demande jamais la fac. Sauf quel ques cas qui sont très brillants, mais qui représentent 2 à $3 \%$ du lot. 0 n nous envoie des gens tout juste moyens, et ces gens là, on doit en faire des formateurs qui vont ensuite former à l'Ecole polytechnique. C'est incroyable. O n créel'E cole polytechnique et on supprime l'E cole normale supérieure! Pourquoi I'Ecole polytechnique? On veut qu'elle carbure au niveau de l'E cole polytechnique de France. $\gg 9$
\end{abstract}

Cette réforme a égal ement consacré la séparation entre les formations d'ingénieurs et celles de techniciens supérieurs qui n'ont plus lieu dans les mêmes établissements. Des Instituts supérieurs d'études technologiques (ISET) ont été créés en 1992. Ils proposent des formations initiales de techniciens supérieurs d'une durée de 2 ans et demi, réparties en cinq semestres finalisés, dans des spécialités industrielles (génie électrique, électronique, mécanique, etc.) et tertiaires (informatique, technique de commercialisation etc. ${ }^{91}$. L'existence du principe d'un écart de deux ans et demi entre le titre d'ingénieur et celui de technicien supérieur engendre une claire distinction des positions professionnelles de ces deux catégories ${ }^{92}$.

Pourtant, dans ce nouveau paysage du système de formation, un établissement demeure atypique: I'Institut national des sciences appliquées et de technologie (INSAT). Cette institution, qui fonctionne depuis 1996, apparaît largement comme l'œuvre de l'ancien président de l'université de Tunis, Mohamed Amara. Sa construction a été financée par la coopération française et sa création a suscité l'opposition des promoteurs du DNI. En effet, cet établissement n'entrait pas dans le cadre défini par la réforme de $1991^{93}$. II s'agit d'un institut qui forme successivement des techniciens supérieurs en 3 ans, puis des ingénieurs en 5 ans et demi. A près deux ans et demi d'études dans la filière de techniciens supérieurs, les étudiants désirant suivre le cursus de l'ingéniorat doivent réussir aux examens du semestre de confirmation. Un tiers environ des étudiants peut être autorisé à suivre le cycle d'ingénieur.

\title{
CONCLUSION
}

On voit ainsi combien le paysage des établissements délivrant un diplômed'ingénieur s'est largement complexifié depuis la naissance de l'ENIT, à la fin des années 1960. La volontéd'accroîtrelenombre d'ingénieurs en Tunisiea contribuéà multiplier les écoles, tout en faisant émerger la question de la qualité de la formation dispensée aux étudiants. 
Un autre constat de ce panorama peut être fait à partir des transformations du système de formation des ingénieurs en Tunisie : il s'agit de la prégnance de la figure de I'ingénieur d'Etat. La valorisation du titre scolaire a été d'autant plus forte que pendant plus de trente ans la plupart des ingénieurs ont été employés dans les administrations et les entreprises publiques; et que le grade auquel le cadre technique entrait dans la fonction publique, ainsi que son plan de carrière, était déterminé par le diplôme obtenu. Par un effet hystérésis ${ }^{94}$, c'est-à-dire en raison d'un décalage temporel entre la cause et l'effet, l'un des principaux enjeux de la réforme du cursus de l'ingéniorat a concerné la suppression des diplômes définissant justement le profil de carrière de l'ingénieur d'Etat, alors que le recrutement dans l'administration se fermait aux ingénieurs.

Cette réforme a privilégié le modèle français de «grande école », en instituant des établissements qui recrutent par concours à l'issue de classes préparatoires, tandis que ce mode de sélection touche dorénavant en France une minorité d'ingénieurs débutants (de l'ordre de $30 \%$ ) : en effet, l'un des piliers fondamentaux du modèle français de l'ingénieur, à savoir uneformation initiale séparant nettement les écoles et les universités, est justement mis en cause par le développement des « écoles universitaires d'ingénieurs », la croissance des flux de formation universitaire de niveau bac +5 débouchant sur des emplois d'ingénieurs et la création des Instituts universitaires professionnels délivrant un diplôme à bac $+4^{95}$.

La force symbolique de cette référence extérieure entretient une propension très répandue chez les responsables du système éducatif à enfermer les ingénieurs dans des catégories rigides. Elle contribue ainsi à maintenir avec force la vision d'une dichotomie entre «l'ingénieur technique » et «l'ingénieur de conception ». Le premier serait versé dans le process (I'acte de production) qu'il a vocation à améliorer, tandis que le second élaborerait les études de faisabilité et jouerait un rôle déterminant dans la gestion de l'entreprise ${ }^{96}$. La valorisation du titre scolaire a également partie liée au fait que I'ingénieur était et est encore largement perçu par les autorités des Etats issus de la décolonisation comme l'agent du développement industriel et le détenteur du « secret » de la modernité. Le diplôme est incontestablement le sésame qui donne une légitimité aux visées technocratiques des ingénieurs tunisiens.

Par ailleurs, la réforme de l'ingéniorat met en exergue la question la capacité de l'actuel système de formation tunisien à améliorer tant le niveau de qualification des ingénieurs qu'à favoriser la compétitivité des entreprises. Lors de la construction de leur programme de formation, les concepteurs de l'ENIT, tout comme les enseignants, étaient 
en prise avec le monde économique ${ }^{97}$. L'éloignement progressif de ces derniers des centres de décision, a contribué à créer hiatus entre les contenus des enseignements et l'environnement économique. Certes, le discours de la réforme fait référence aux évolutions économiques, mais il ignore le fonctionnement le marché du travail des ingénieurs. La réforme n'a pas été précédée d'études sur les compétences exigées pour répondre aux besoins de l'économie tunisienne.

Cette démarche s'explique largement par l'existence d'un système d'enseignement supérieur régulé uniquement par l'offre de formation. Autrement dit, les demandes exprimées par les étudiants et les acteurs du secteur productif sont largement ignorés au moment de la construction du contenu des programmes. Dans le cadre d'une économie protégée, dominée par le secteur public et dont les entreprises se contentent de proposer des biens et des services de médiocre qualité, le fonctionnement de ce système de régulation permet d'insérer les diplômés sur le marché du travail. C'est de moins en moins le cas aujourd'hui, où la Tunisie a signé un accord de libre échange avec l'Union européenne. Le secteur privé, qui exprime de nouvelles exigences en terme de compétence, est appelé à prendre à la relève des entreprises publiques et par conséquent à devenir progressivement le principal employeur des ingénieurs ${ }^{98}$.

\section{N OTES}

1 BENSALEM Lilia, « La profession d'ingénieur en Tunisie. Approche historique », in LONGUENESSE Elisabeth (dir.), Bâtisseurs et bureaucrates. Ingénieurs et société au M aghreb et au M oyen-O rient, Lyon, Maison de l'Orient, 1990, p. 92.

2 Un résumé de ces travaux sociologiques se trouve dans BOUFFARTIGUE Paul et GADEA Charles, «Les ingénieurs français. Spécificités nationales et dynamiques récentes d'un groupe professionnel », Revue française de sociologie, XXXVIII, p. 305. Ces deuX auteurs décrivent également trois autres modèles d'organisation du travail technique auxquels se rattachent divers pays développés.

3 BOURDIEU Pierre, La noblesse d'Etat. Grandes écoles et esprit de corps, Paris, Editions de Minuit, 1989, p. 188 et pp. 198-199.

4 BEN SALEM Lilia, D éveloppement et problème de cadres, le cas de la Tunisie Un exemple : les cadres supérieurs del'économie tunisienne, Tunis, Cahiers du CERES, 1976, (série sociologique), p. 65.

5 II convient pourtant d'indiquer que la Tunisie est le pays du Maghreb qui, durant la colonisation, a vu le plus grand nombre d'ingénieurs «autochtones » formés en France. Si dans l'entre-deux-guerres les premiers ingénieurs tunisiens ont eu toutes les peines du monde à être embauchés dans les services techniques del'administration du Protectorat, en revanche, après la Seconde Guerre mondiale, en raison des nécessités de la reconstruction, les autorités coloniales ont été obl igées de recruter des cadres locaux.

6 BEN SALEM Lilia, «Les ingénieurs tunisiens au XIXe et XXe siècles », Revue de la M éditerranée et du M onde musulman , 72, 1994/2, p. 68.

${ }^{7}$ MAGNIN J.G., «La profession d'ingénieur en Tunisie », Ibla, n 98, second semestre 1962, p. 189. 
8/bidem, p. 189.

${ }^{9}$ La Tunisie se serait vu refuser sa demande de financement de l'école d'ingénieurs sous prétexte que les Tunisiens sont plus des commerçants que des bâtisseurs, entretien avec M okhtar Latiri, septembre 2001.

10 LATIRI Mokhtar, «Libres propos sur l'origine de I'ENIT », conférence prononcée à l'occasion du 30" anniversaire del'EN IT , 12 décembre 1998, document aimablement fourni par Mokhtar Latiri.

11 En août 1968, A hmed Ben Salah installe également le conseil scientifique de l'ENIT et annonce la nomination par le président Bourguiba du directeur de l'établissement, Mokhtar Latiri, entretien avec Mokhtar Latiri, septembre 2001.

12 Ibidem.

13 La Jaune et la Bleue, $n^{\circ}$ 5, mai-juin 1973. «Pour moi la filière A préparait en 8 ans et plus les $5 \%$ des meilleurs élèves tunisiens. II était prévu qu'ils épuisaient le pipe line français, faisaient leur école d'application et allaient s'inscrireen doctorat ou en Ph.D. L'exemple par excellence de cette filière est le professeur Farouk Kammoun : lauréat de Supelec, il s'est inscrit aux E tats-U nis à U CLA », entretien avec Mokhtar Latiri, septembre 2001. En fait, la filière A a surtout consisté à envoyer les meilleurs bacheliers tunisiens dans les grandes écoles françaises, M okhtar Latiri se chargeant du placement professionnel en Tunisie des diplômés de retour de France.

14 «Cette démarche, commel'affirme A nousheh Karvar, explique sans doute le contingent important d'élèves tunisiens à l'Ecole polytechnique française. Elle pourrait servir également d'explication à l'établissement professionnel des jeunes polytechniciens dans leur pays d'origine». KARVAR, La formation

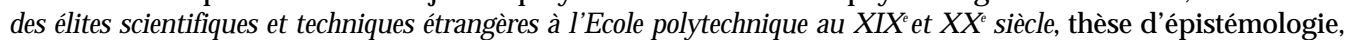
d'histoire des sciences et des techniques, université Paris VII, décembre 1997, p. 277.

15 Entretien avec Mokhtar Latiri, op. cit.

16 La Jaune et La Bleue, « L'ENIT en 1975 », p. 20, document aimablement fourni par Mokhtar Latiri. La première promotion del'ENIT (1973) concerneles diplômés dela filièreD, Entretien avec Mokhtar Latiri, op. cit.

17 KARVAR A nousheh, op. cit., p. 276.

18 En 1975, le corps enseignant de l'ENIT est composé de 131 Français (47 à temps plein et 84 à temps partie) de 54 Soviétiques ( 53 à temps plein et 1 à temps partiel), de 55 Tunisiens (29 à temps plein et 26 à temps partiel) et de 11 ressortissants d'autres pays, La Jaune et La Bleue, «L'ENIT en 1975 », op. cit., p. 74. L'ENIT se caractérise par la lenteur du remplacement des effectifs d'enseignements étrangers par des Tunisiens (ce n'est qu'à partir du milieu des années 1980 que ces derniers deviendront majoritaires). Cette évolution tient en grande partie au démarrage plus tardif des activités de I'ENIT et à la captation des enseignants par les facultés des sciences. Pour plus de détails, voir à ce sujet SIINO François, «La construction du système universitaire tunisien. Flux croisés et importation des pratiques scientifiques », in GEISSER Vincent (dir.), D iplômés maghrébins d'ici et d'ailleurs. Trajectoires sociales et itinéraires migratoires, Paris, CNRS, 2001, pp. 83-84.

19 Cet article s'appuie en partie sur l'exploitation statistique de la base de données des inscrits au tableau de l'Ordre des ingénieurs tunisiens. Ces derniers constituent la population mère d'une enquête national e par questionnaire financée par I'Institut français de coopération et menée auprès d'un échantillon représentatif de 1000 ingénieurs tunisiens. Pour construire cet échantillon, nous avons réaliséles opérations techniques de codification de l'information relatives aux variables figurant dans le fichier de l'Ordre: âge ; lieu denaissance ; pays et spécial itédeformation ; date de sortie de l'école; fonction occupée, etc. Par la suite, l'échantillon a été stratifié en fonction du pays de formation et de la distribution des ingénieurs dans les différentes branches d'activité de l'économie tunisienne.

20 Source : Base de données de l'Ordre des ingénieurs, 1999. Cette organisation regroupe presque 10000 ingénieurs tunisiens sur un total d'environ 20000 recensés par l'Institut national de la statistique. Voir BEN SEDRINE Saïd et GOBE Eric, Trajectoires sociales et insertion professionnelle des ingénieurs en Tunisie, rapport de pré-enquête, IRMC et INTES, septembre 2000.

21 Pour la différence entre ces deux grades, voir infra

22 HADHRI Taïeb, «Ingénierie de la formation de l'ingénieur. Expérience tunisienne 1969-1999 », $L^{\prime}$ 'ingénieur tunisien, $\mathrm{n}^{\circ} 21, \mathrm{p} .37$.

23 LATIRI Mokhtar, «Des ingénieurs... Pourquoi faire ? », Journées de l'ingénieur, 21 octobre 1994, document aimablement fourni par l'auteur. 
${ }^{24}$ La jaune et la Bleue, «L'ENIT en 1975 », op. cit., p. 13.

25 Pour éviter toute confusion nous n'utilisons pas dans ce texte le titre «d'ingénieur diplômé » mais le grade d'ingénieur principal pour désigner les titulaires d'un bac +6 .

26 BOUfFA RTIGUE Paul, Les cadres. Fin d'unefigure sociale, La Dispute, Paris, 2001, p. 127.

27 ZAGHOUNDA Habib, «L'Ecole supérieure d'agriculture de Tunis », in I'IN A T : un siècle sur la voie de l'excellence, ministère de l'A griculture, 1998, p. 43.

28 Seuls les enseignants et les chercheurs reprendront leur poste.

29 Le nombre d'étudiants formés par l'établissement augmente progressivement : de 10 en 1960, 21 en 1963, et 35 en 1965, les promotions passent à une quarantaine de diplômés entre 1967 et 1971, ZA GHOUNDA Habib, op. cit, p. 35.

30 JERRA YA A bderrahmen, «Parcours de l'Ecole depuis l'indépendance en raccourci », in I'IN A T...., op. cit., p. 28

31 SKOURI M ohamed, «L'épisode faculté d'agronomie : chronique d'une période de turbulences », in I'IN A T ... , op. cit., p. 51.

32 Voir JERRAYA A bderrahmen, op. cit. , p. 28. Le recrutement par l'ENSAT d'ingénieurs agronomes formés en France permet de fournir l'encadrement nécessaire au fonctionnement de la deuxième année du troisième cycle.

33 SKOURI Mohamed, «L'épisode faculté d'agronomie : chronique d'une période de turbulences », in I'INA T ... , op. cit., 1998, p. 47. Attaché au ministère de l'Agriculture, l'enseignement supérieur agricole passe sous la tutelle du ministère de l'Education nationale. Ce passage, dans l'optique d'une mobilisation centralisée des ressources du pays, vise dans le discours officiel à mettre l'ensemble des structures de l'enseignement supérieur au service du développement de la Tunisie.

34 Ce rétablissement du régime des études se situe dans la logique administrative créée par la mise en place de I'ENIT. Deux décrets de 1971 et de 1972 organisent le statut des cadres techniques de la fonction publique et fixent la durée des études donnant accès aux grades d'ingénieur adjoint (c'est-à-dire un grade réservé aux titulaires d'un diplôme de technicien supérieur) - 2 ans, d'ingénieur des travaux del'Etat - 4 ans et d'ingénieur principal - 6 ans. BELKHODJA Kamel, «Les difficultés d'une renaissance », in I'INAT ... , op. cit. , p. 56.

35 Un diplôme d'ingénieur spécialisé est décerné à ceux qui ont réussi un concours et suivi un cycle d'une année de spécialisation et d'une année d'application.

36 BELKHODJA Kamel, op. cit., p. 56.

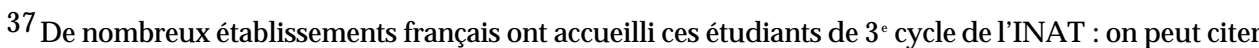
entre autres, I'INA Paris-Grignon, I'Ecole nationale du génie rural des eaux et forêts de Paris (ENGREF), Ecole nationale supérieure d'agronomie et des industries alimentaires de Nancy (ENSAIA), le Centre international des hautes études agronomiques méditerranéennes (CIHEAM), les Ecoles nationales supérieures d'horticulture (ENSH), ibidem., p. 59.

38 A partir du milieu des années 1970, I'INAT forme des promotions d'environ 60 ingénieurs techniciens et de 20 à 40 ingénieurs principaux (sans compter les spécialités sous traitées en totalité à l'étranger). Pour davantage de précisions voir JERRAYA Abderahmen, «Le temps de la maturité », in I'IN AT..., op. cit., p. 92. cit. , p. 265

39 BELKHODJA Kamel, «Principaux traits de l'agriculture après l'indépendance », in l'IN AT ... , op.

40JERRAYA A bderrahmen, «Le temps de la maturité», in I'IN A T, op. cit. , p. 84.

41 Source : Enquête S. Ben Sedrine \& E. Gobe, 2000.

42 TISSAOUI Tahar, «L'ingénieur dans l'agriculture. A perçu sur les évolutions durant un siède de formation », L'ingénieur tunisien, $\mathrm{n}^{\circ} 21$, p. 42.

43 BACCOUCHE Chokri, «Chômage des ingénieurs : cinq scénarios pour une solution globale », Réal ités, $n^{\circ} 745,30$ mars 2000. 
44 Source : Base de données de l'Ordre des ingénieurs, 1999.
45 L'enseignement et la formation en chiffres (1989, 1994 et 2000), ministère de l'Enseignement supérieur,
Tunis.
46 «A I'ENIT, affirme Mokhtar Latiri, les enseignants sou haitent que la moitié des étudiants oublient de se lever. D e cette façon, ceux qui viennent en cours peuvent trouver une place dans l'amphithéâtre. $M$ al heureusement, on ne maîtrise pas cette montée des classes jeunes », op. cit.

47 ZOUARI-BOUATTOUR Salma et alii, Financement de l'éducation, Etudestratégiquen ${ }^{\circ} 20$, ministère de I'Education et ministère de l'Enseignement supérieur, Tunis.

48 BEN SEDRINE Saïd et GEISSER Vincent, Le retour des diplômés. Enquête sur les étudiants tunisiens formés à l'étranger : Europe, A mérique et M onde arabe, Centre de Publication Universitaire, Tunis, 2001, p. 24.

49 Ibidem, pp. 24-25.

$5064 \%$ des ingénieurs inscrits à l'Ordre des ingénieurs ont le statut d'ingénieur technicien contre 36\% celui d'ingénieur principal.

51 BEN SEDRINE Saïd et GEISSER Vincent, Le retour des diplômés, op. cit., p. 8.

52 Mokhtar Latiri, ardent défenseur de la filière A, déplore le non-retour des ingénieurs tunisiens formés en France et dans d'autres pays occidentaux, op. cit.

53 Le document de l'ATUGE comprend les ingénieurs répertoriés par l'association à partir des annuaires et des listes de diplômés tunisiens fournis par les grandes écoles.

54 BAHLOUL Nizar, «Etudiants tunisiens à l'Ecole polytechnique de Paris. Fleurons d'une jeunesse à la tête bien faite», Réalités, n 768, 14 septembre 2000, p. 39.

55 Nous n'avons pas comptabilisé les diplômés des grandes écoles de commerce (HEC, ESSEC et les Ecoles supérieures de commerce). Pour la signification des sigles, voir l'annexe.

56 Nous les avons comptés dans la mesure où la plupart sont chargés de cours ou disposent du statut d'attaché temporaire d'enseignement et de recherche.

57 Nous avons repéré 72 centraliens qui ont obtenu leur diplôme entre 1990 et 1999.

58 Pour ce qui concerne l'EN SITM, il est impossible de tirer des conclusions car dans 32 cas sur 36, le pays d'emploi n'est pas mentionné.

59 Pour certaines écoles, on ne connaît pas dans de très nombreux cas le lieu de travail des diplômés.

60 II faut rajouter à ce chiffre 2 polytechniciens travaillant respectivement en Grande-Bretagne et aux Etats-Unis. Par ailleurs, on a repéréle pays d'emploi de 10 centraliens sur 18 (6en France et 4 en Tunisie). Du côté des Ponts et Chaussées, les données sont fragmentaires: dans 18 cas il n'a pas été possible de déterminer le lieu del'emploi. Cependant, il convient de noter que sur les 11 personnes repérées, 10 travaillent en Tunisie contre 1 en France.

${ }^{61}$ C'est tout au moins ce qui ressort de l'entretien que nous avons eu avec le président de l'ATUGETunisie qui a expliqué ainsi son retour et celui de certains de ses condisciples de l'Ecole centrale de Lyon, Entretien avec Talel Chérif, août 2001.

62 SIINO François, Science et pouvoir dans la Tunisie contemporaine. Contribution à une analyse de la politique scientifique tunisienne (1956-1996), Institut d'études politiques, université Aix-Marseille III, octobre 1999, pp. 360-361.

63 Ibidem, p. 361

64 HADHRI Taïeb, op. cit. , p. 36.

65 Ibidem, pp. 35 et 36.

66 Entretien avec Tahar Belakhdar, octobre 2000 et juillet 2001. M. Tahar Belakhdar, a été, entre autres, directeur de l'ESPTT et du collège préparatoire de I'ENIT. 
67 Ibidem. En 1986, le cycle préparatoire de l'ENIT a été délocalisé à Nabeul avec la création d'un institut préparatoire dans cette même ville.

68 Entretien avec Taïeb Hadhri (X - 1977), actuel recteur del'académie de Tunis et ancien directeur de I'Ecole Polytechnique de Tunis, janvier 2001. On retrouve le mêmeargumentaire chez A bderrahmen Jarraya,

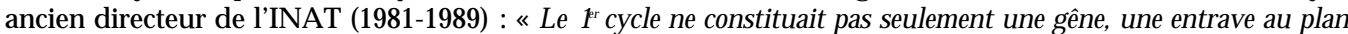
budgétaire, sa coexistence avec les autres cycles était dans bien des cas, à l'origine de perturbations, de manifestations et de grèves qui ont émaillé de temps à autre toute la décennie. Les étudiants avaient pris l'habitude de prendre en "otage" I'Amphi de l'année préparatoire pour déclencher leurs mouvements de contestation. »JERRAYA A bderrahmen, «Le temps de la maturité », op. cit., pp. 72-73.

69 Entretien avec Tahar Belakhdar, op. cit., «Le système de I'O rientation ne doit pas être à l'origine du recrutement dans telle ou telle école. Pour moi, les écoles sont à l'instar des crus devin, chacune a sa valeur et les élèves doivent se bagarrer pour entrer dans la meilleure. En plus, avec des préparations séparées, on introduit une émulation entre les écoles d'ingénieurs et tout le monde y trouve son compte. C'est déjà ce qu'on aurait dû faire dans les années 1980, avec l'expérience de l'Institut préparatoire de $\mathrm{N}$ abeul. M ais les résistances locales et le changement de ministre ont empêché que le projet voit le jour. A u bout du compte, on s'est retrouvé devant une monstruosité. On pensait créer un système de préparation et on avait simplement déménagé le collège préparatoire de I'EN IT, point à la ligne L'avantage deN abeul par rapport à l'EN IT résidait dans le fait que les élèves étaient bien logés dans un cadreagréable. »

70 Entretien avec Taïeb Hadhri, op. cit.

71 Entretien avec Tahar Belakhdar, op. cit., «J'ai exposé mon projet devant un comitédes sages convoqué par le ministre de l'Education. II y avait dans ce comité les plus grands ingénieurs du pays : des polytechniciens, des centraliens d'une soixantaine d'année. Je leur ai dit qu'il ne fallait pas quel'on resteavec un système bâtard qui n'existe nul part ; il faudrait que l'on adopte un système, mais à $100 \%$. Si c'est le système français on doit le faire à $100 \%$. N os jeunes adorent faire des "prépa" en France. M ais après tout qu'est-ce qu'une "prépa" ? Ce sont des sciences que nous maîtrisons. N ous avons des bons mathématiciens et des bons physiciens. Si nous choisissons ce système, choisissons-le à $100 \%$. J'ai cité l'exemple du M aroc qui avait démarré des "prépa" qui commençaient à faire réussir des élèves à Polytechnique. A la suite de mon exposé, il y a eu un débat, il y avait des ingénieurs pour et d'autres qui étaient contre Le ministre a arbitré en faveur du projet. Un mois plus tard la commission d'étude était créée. »

72 Pour tous les détails voir VERMEREN Pierre, La formation des élites par l'enseignement supérieur au $M$ aroc et en Tunisie au X X ${ }^{\circledR}$ siècle, thèse d'histoire, université Paris VIII Saint Denis, 2000, pp. 687-688.

73 Entretien avec Tahar Belakhdar, op. cit.

${ }^{74}$ Les copies tunisiennes étaient corrigées anonymement en France.

75 Entretien avec Taïeb Hadhri, op. cit.

76 Cet établissement est situé dans la banlieue résidentielle de Tunis à la Marsa.

77 Entretien avec Tahar Belakhdar, op. cit. Cette règle a rapidement été abandonnée. Les élèves de I'IPEST réussissant à des concours d'écoles moins prestigieuses ont pu ainsi bénéficier de bourses. Situation qui selon certains responsables de la coopération française est source de concurrence pour les établissements tunisiens. En fait, la France donne des bourses aux lauréats des concours des très grandes écoles, alors que la Tunisie aide financièrement les autres.

78Ceci au plus grand dam du promoteur du projet : «Je pensais quele système del'IPEST serait généralisé à l'ensemble des "prépa". Je croyais que les élèves des autres "prépa" seraient choisis sur dossier. Le projet s'est arrêté avec le départ du ministre de l'E ducation. Plus personne ne s'intéressait à ce dossier », ibidem.

79 Le ministère de l'Education et des sciences a créé en 1993 un Comité de rénovation des études d'ingénieur (CREDIT) avec pour mission de valider les cursus de formation conduisant au DNI.

80 Mohamed Jaoua est docteur en mathématiques et enseigne actuellement à I'ENIT. II a étéle premier directeur de l'Ecole polytechnique de Tunis.

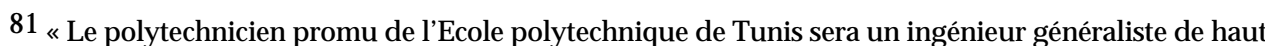
niveau, susceptible de maîtriser la technologie et de la faire progresser, de conduire les grands projets et d'animer les équipes pluridisciplinaires qu'ils impliquent. II est appelé donc à assumer d'importantes responsabilités dans le développement scientifique, industriel et économique du pays. », interview de Mohamed Jaoua, in «Les enjeux d'une grande école», L'E conomiste maghrébin, n 103, 13-26 avril 1994, p. 11. 
82 Entretien avec Taïeb Hadhri, op. cit.

83 Les élèves de l'EPT résident sur place dans des chambres individuelles situées dans un minicampus entièrement câblé et équipé d'installations sportives. Ibidem.

84 BEN SEDRINE Saïd et GEISSER Vincent, Le retour des diplômés... , op. cit., pp. 44-45. Le système tunisien n'accorde qu'une faible importance au premier choix du bachelier. En 1994, le taux moyen de satisfaction des bacheliers par rapport à leur orientation universitaire était compris entre $20 \%$ \& $50 \%$. Les plus faibles taux de satisfaction concernaient les candidats à une formation en gestion $(28,1 \%)$ et en sciences médicales (29,1\%).

85 «Les "prépa” tunisiennes accueillent aujourd'hui des élèves qui n'ont ni la moyenne en math, ni la moyenne en physique », entretien avec Tahar Belakhdar, op. cit.

86 Ibidem. science.

87 Justement tous ne sont pas indiqués, notamment ceux de l'IPEI de Sousse et des facultés de

88 Si I'on se réfère au baccalauréat de sciences expérimentales, moins prestigieux que celui de mathématiques, on a affaire à une hiérarchie quelque peu différente. Les IPEI sont mieux positionnés.

89 Entretien avec Taïeb Hadhri, op. cit.

90 Entretien cité in SIINO François, op. cit., p. 423.

91 BEN SEDRINE Saïd et GEISSER Vincent, «Les diplômés à la sortie de l'Université. Devenir social et stratégies d'insertion professionnelle », M aghreb-M achrek, $n^{\circ}$ 157, juil let-septembre 1997, p. 27. Les lauréats des ISET peuvent accéder en première année des écoles d'ingénieurs après étude de leur dossier.

92 Tahar Belakhdar tire un bilan assez mitigé des réformes qu'il a contribuéà finaliser. Il en attribue l'échec relatif au fait que les enseignants titulaires sont exclusivement des universitaires et que le statut des agrégés n'ait pas été revalorisé : «On a créé un nouveau corps d'enseignants, les technologues. Ils ne sont plus recrutés à partir de thèses mais à partir d'agrégations de génie électrique, génie mécanique, génie civil avec un statut assez intéressant leur permettant de progresser et de rester en contact avec le monde professionnel (...). . M ais le statut des agrégés est en train de se casser la figure. On a oublié de revaloriser leur salaire. Les agrégés fuient et sont en train de faire des thèses, ils veulent intégrer I'université classique afin d'avoir de meilleures conditions matérielles », op. cit. II est à noter que Mokhtar Latiri regrette que le statut de l'ingénieur enseignant ne soit pas valorisé. Entretien..., op. cit.

93 Cette situation fait dire à Mokhtar Latiri que l'IN SAT est condamné à faire son aggiornamento, car ses statuts sont en contradiction avec la législation qui réglemente les écoles d'ingénieurs délivrant le DNI, ibidem. De son côté, son directeur actuel, Mekki Ksouri, insiste sur l'utilité et l'apport d'un établissement comme I'INSAT dans le système de formation tunisien : «A vec le DN I, on a voulu s'aligner sur ce qui se fait en France. A mon avis dans la formation d'ingénieur, on a besoin d'expérimenter beaucoup de systèmes. A un certain moment en Tunisie, il existait la filière moyenne et la filière longue II est vrai que l'instauration d'un diplôme unique était nécessaire pour résoudre le problème des carrières dans la fonction publique. $M$ ais pour faire un bon produit ingénieur, il y a plusieurs façons. C'est pour cette raison que l'existence de I'IN SAT est très positive. En France, I'ingénieur est un bac +5 , mais pour y arriver, il existe plusieurs formules. V ous avez la formule "prépa" +3 ans, mais vous avez aussi des formations intégrées qui se trouvent partout, dans les Instituts nationaux des sciences appliquées (IN SA ). Le fait de passer plus de 5 ans au même endroit est positif pour la qualité de la formation », entretien avec Mekki Ksouri, février 2001.

94 BOURDIEU Pierre, La noblessed'Etat..., op. cit., pp. 20 et 301.

95 BOUfFARTIGUE Paul et GADEA Charles, «Un héritage à l'épreuve. Bref panorama des évolutions dans la formation et l'emploi des ingénieurs en France », Formation emploi, $n^{\circ}$ 53, janvier-mars 1996, p. 9.

96 Interview de Mohamed Jaoua, op. cit., p. 12.

97 Entretien avec A bdelaziz Halleb.

98 Voir BEN SEDRINE Saïd, « Gagnants et perdants de la transition libérale, l'insertion professionnelle des diplômés en Tunisie », in GEISSER Vincent (dir.), Diplômés maghrébins d'ici et d'ailleurs..., op. cit., pp. 105-121. 


\section{Annexe: sigles des écoles d'ingénieurs en France}

\begin{tabular}{|c|c|}
\hline EC Lille & Ecole centrale de Lille \\
\hline EC Lyon & Ecole centrale de Lyon \\
\hline EC Paris & Ecole centrale de Paris \\
\hline ENAC & Ecole nationale de l'aviation civile \\
\hline ENPC & Ecole nationale des ponts et chaussées \\
\hline ENSUIm & Ecole normale supérieure UIm \\
\hline ENSAE Paris & Ecole nationale supérieure de l'administration et de l'économie \\
\hline ENSAI & Ecole nationale de la Statistique et de l'analyse de l'information \\
\hline ENSAM & Ecole national e supérieure des arts et métiers \\
\hline ENSAT & Ecole nationale supérieure agronomique de Toulouse \\
\hline ENSBANA & $\begin{array}{l}\text { Ecole national e supérieure de biologie appliquée à la nutrition et à } \\
\text { l'al imentation }\end{array}$ \\
\hline ENSEA & Ecole nationale supérieure d'électronique et de ses applications \\
\hline ENSEEG & $\begin{array}{l}\text { Ecole nationale supérieure d'électronique et d'électrométallurgie de } \\
\text { Grenoble }\end{array}$ \\
\hline ENSEEIHT & $\begin{array}{l}\text { Ecole nationale supérieure d'électronique, électronique informatique, } \\
\text { hydraulique deToulouse }\end{array}$ \\
\hline ENSEM & Ecole nationale supérieure d'électricité et de mécanique de N ancy \\
\hline ENSER (Grenoble) & $\begin{array}{l}\text { Ecole nationale supérieure d'électronique et de radioélectricité de } \\
\text { Grenoble }\end{array}$ \\
\hline ENSHMG & Ecole nationale supérieure d'hydraulique et de mécanique de Grenoble \\
\hline ENSI (Caen) & Ecolenationale supérieure d’ingénieurs de Caen \\
\hline ENSIA & Ecole nationale supérieure des industries agricoles et alimentaires \\
\hline ENSIEG & Ecole nationale supérieure d’ingénieurs électriciens de Grenoble \\
\hline ENSIMAG & $\begin{array}{l}\text { Ecole nationale supérieure d'informatique et de mathématiques } \\
\text { appliqués }\end{array}$ \\
\hline ENSITM & Ecole nationale supérieure des industries textiles de Mulhouse \\
\hline ENSMM & Ecole nationale supérieure de mécanique et de microtechnique \\
\hline ENSP (Grenoble) & Ecole nationale supérieure de physique de Grenoble \\
\hline ENSP (Marseille) & Ecole nationale supérieure de physique de Marseille \\
\hline ENSTA & Ecole nationale supérieure de techniques avancées \\
\hline ESIM & Ecole supérieure des ingénieurs de Marseille \\
\hline ESTP & Ecole supérieure des travaux publics \\
\hline IIE & Institut d'informatique d'entreprise \\
\hline INAPG & Institut national agronomique Paris-Grignon \\
\hline INSA (Lyon) & Institut national des sciences appliquées de Lyon \\
\hline INSA (Rouen) & Institut national des sciences appliquées de Rouen \\
\hline INSA (Toulouse) & Institut national des sciences appliquées de Toulouse \\
\hline INT & Institut national des téécommunications \\
\hline Mines de Nancy & Ecole des mines de Nancy \\
\hline Mines de Paris & Ecole des mines de Paris \\
\hline Mines de St-Etienne & Ecole des mines de Saint-Etienne \\
\hline SUPAERO & Ecole nationale supérieure de l'aéronautique et de l'espace \\
\hline SUPELEC & Ecole nationale supérieure d'électricité \\
\hline TELECOM Bretagne & Ecole nationale supérieure de télécommunications de Bretagne \\
\hline TELECOM Paris & Ecole nationale supérieure de télécommunications \\
\hline & \\
\hline
\end{tabular}


Le modèle des élites techniques en question 



\section{La formation des élèves algériens, tunisiens et marocains à l'Ecole polytechnique française (1921-2000) : des acteurs de l'histoire aux «élites de peu »}

A nousheh KARVAR

\section{LES ÉLÈVES ÉTRANGERS À L'ÉCOLE POLYTECHNIQUE}

A partir de 1921, date à laquelle des candidats étrangers ont été autorisés à présenter, au même titre que les ressortissants français, le concours d'entrée de l'Ecole polytechnique, la répartition des lauréats par pays et par aires géopolitiques (Europe occidentale et Amérique du Nord / Europe centrale et de l'Est / anciennes colonies et protectorats / Proche-Orient / Asie / Amérique du Sud) révèle une prédominance des anciennes colonies et protectorats de la France ${ }^{1}$. Les décolonisations des années 1950 et 1960 se traduisent, en effet, par une arrivée importante d'élèves en provenance du Viêtnam, delaTunisie, du Maroc, du Liban et del'Algérie. Parallèlement au grand contingent des élèves maghrébins, nous observons également un groupe d'élèves que nous appellerons des «étrangers de passage ». Ils ne font pas partie d'un flux important vers la France comme dans le cas des étudiants maghrébins par exemple, mais ce sont des cas isolés qui se trouvent de toute évidence en France pour des raisons contingentes (Norvégien, Suédois, Autrichien, Indonésien, Chilien...). On compte enfin parmi les élèves originaires du bassin méditerranéen, un certain nombre d'élèves juifs ayant suivi une démarche identique de naturalisation et d'installation en France, pour des raisons cette fois liées au sort réservéà leur communauté dans les pays du Maghreb et du ProcheOrient.

Les indépendances des années 1950 et 1960 exposent très rapidement l'établissement parisien à la question du concours qu'il pourrait - ou devrait - apporter à l'instruction technique de la relève locale et qui débouche, au cours des décennies suivantes, sur la fuite des cerveaux de ces pays vers l'ancienne métropole. Le débat s'articule autour de deux axes : d'une part, on s'interroge sur la meilleure manière 
d'attirer vers l'Ecole les «vrais »étrangers, « d'un certain niveau », d'origine européenne ou américaine, et qui retourneraient dans leur pays à la fin du séjour en France, contribuant ainsi au rayonnement de l'Ecole ${ }^{2}$. C'est en leur faveur qu'on délibère au conseil de perfectionnement sur la revitalisation du statut d'auditeur à la fin des années 1970. La discussion porte, d'autre part, sur les «faux » étrangers, ressortissants des anciennes colonies et protectorats, issus du système d'enseignement français et désireux de s'installer en France. Il s'agit d'envisager, d'une part, une possible limitation de leur nombre, d'autre part une assimilation de plus en plus complète de leur statut à celui des Français, notamment par l'élimination des régimes de faveur au concours et par l'élévation de la barre d'admission.

La question de l'admission d'un type d'étrangers, en qualité d'auditeurs externes, est mise à l'ordre du jour du conseil de perfectionnement pour la première fois en 1959. Elledonnelieu à un long échange de vues. On convient d'approfondir le sujet « sans hâte » et de réfléchir aux modalités de l'examen à faire subir éventuellement aux candidats 3 .

En 1963, ce sont les élèves internes qui font l'objet de l'attention du conseil. II s'agit d'abord de rapprocher le plus possible les conditions dans lesquelles les étrangers passent le concours général de celles des candidats français. Six mois plus tard, on tente de donner une seconde vie au statut d'auditeur libre, créé à la fin du XIX e siècle pour formaliser la présence des étrangers qui suivraient l'enseignement de l'Ecole en auditeur libre en les soumettant à un examen d'entrée (aptitude en langue française et instruction scientifique), au contrôle de présence et aux interrogations générales.

Entièrement tombé en désuétude depuis l'instauration du concours commun aux candidats français et étrangers, ce statut s'adresserait vaguement aux étrangers qui, « attirés par le renom de l'Ecole », étaient aptes «à profiter de son enseignement grâce à des connaissances d'un niveau comparable... », mais ne donnait pas accès au titre d'ingénieur polytechnicien. Ce n'est qu'un an plus tard que le directeur des études tente de préciser la population ciblée. II rappelle que le statut d'auditeur libre est réservé aux candidats ayant suivi un enseignement «tout à fait différent » à l'étranger, excluant ainsi tous ceux qui auraient suivi une classe de mathématiques spéciales. Les questions du niveau de connaissances exigées et du futur titre que porteront les anciens auditeurs occupent la majeure partie des discussions du conseil ${ }^{4}$. Ce débat n'est suivi d'aucun effet. II faudra attendre les lendemains de mai 1968 pour voir la question des élèves étrangers de nouveau émerger. Dans la foulée des réformes envisagées à l'Ecole polytechnique, la « commission aval », chargée des questions relatives à l'admission, propose d'augmenter 
le nombre d'élèves étrangers, notamment européens. L'aménagement du concours d'entrée étant écarté par le directeur des études, celui-ci évoque les principales différences entre le système d'enseignement en vigueur aux Etats-Unis (MIT) et en Allemagne (Technische Hochschulen) pour conclure à la difficulté de trouver des passerelles entre des systèmes d'enseignement trop différents. Un point est fait à cette occasion sur l'application du statut d'auditeur libre, pour constater l'absence quasi-totale de candidats pour cette catégorie ${ }^{5}$. Ces obstacles d'ordre structurel n'empêchent pas le conseil d'émettre le vœu de voir l'Ecole polytechnique « devenir une école européenne ».

En 1974, les débats sur le rôle international de l'Ecole polytechnique élargissent leur champ d'application, du recrutement à l'enseignement et au corps enseignant. Ils se déroulent parallèlement aux réflexions sur la modernisation de l'enseignement et sur l'ouverture vers les « pays en voie de développement », compte tenu du « dével oppement considérable » que ces pays promettent à l'époque ${ }^{6}$.

Un an après, c'est le ministre de la Défense qui demande au président du conseil d'administration le doublement du nombre d'élèves étrangers à l'Ecole, dans le cadre d'une politique générale préconisée par l'ensemble des grandes écoles. La question portée cette fois devant la Conférence des grandes écoles ne trouve pas de réponse satisfaisante. D'une part, les écoles d'application n'arriveraient pas à faire face à un nombre plus important de demandes d'admission émanant des élèves étrangers. D'autre part, I'admission d'un plus grand nombre d'élèves étrangers à l'Ecole polytechnique nécessiterait une révision à la baisse de la barred'admission qu'aucun membre du conseil ne souhaite envisager.

Pour grossir le nombre d'étrangers à l'Ecole, l'éternelle solution de recrutement d'auditeurs libres est alors évoquée, accompagnée du même constat d'absence de candidats pour cette catégorie. L'avis du ministère des Affaires étrangères est demandé. Une soixantaine de lettres sont aussi envoyées aux anciens élèves étrangers pour récolter leurs suggestions. On prévoit enfin d'inviter les attachés militaires et les conseillers culturels des missions diplomatiques étrangères à la garden party del'Ecole $\mathrm{e}^{7}$.

Deux ans plus tard, en 1979, devant le constat du non-retour des élèves étrangers dans leur pays d'origine, le conseil d'administration, sur proposition de la « commission aval », approuve I'harmonisation des conditions d'admission des élèves étrangers et français : les épreuves de langues vivantes et d'éducation physique ne sont plus facultatives pour les candidats étrangers. La souplesse est seulement maintenue pour 
I'épreuve de français ${ }^{8}$. La cause de ces nouvelles règles doit être recherchée dans les différences entre les filières d'admission à l'Ecole, la filière réservée aux étrangers étant réputée plus facile et de ce fait, préjudiciable aux Français admis par le concours normal.

Les renseignements collectés dans l'annuaire des anciens élèves confirment le nonretour des élèves étrangers de l'Ecole polytechnique constaté par le conseil d'administration. L'évolution de cette tendance sur la période 1955-85 décèle une attitude différenciée dans le temps chez les ressortissants des pays les plus représentés à l'Ecole 9 . La promotion à partir de laquelle ces élèves ont opté pour l'expatriation est variable selon les pays: promotion 1952 pour le Viêt-nam, 1974 pour le Liban, 1975 pour la Tunisie, 1980 pour le Maroc, et 1977 pour l'A Igérie. Si les événements politiques dans le pays d'origine expliquent ces dates pour certaines nationalités comme le Liban (un retour pour deux jusqu'à la promotion 1974, aucun retour pour les promotions après) ou le Viêt-nam (un retour pour sept jusqu'à la promotion 1952, aucun retour après, sauf un de la promotion 1959), en revanche le cas des pays du Maghreb semble s'inscrire dans la problématique de la «fuite des cerveaux ». Mais, au-delà de cette problématique qu'il faudrait sans doute analyser plus finement dans le contexte propre à chacun de ces pays, on constate que les élèves étrangers originaires des anciennes colonies françaises conservent, dans le discours officiel de l'institution, leur statut de membres de la communauté nationale et ne peuvent revêtir, à cet égard, le « véritable » attribut d'« étranger ».

Malgré ces échecs répétés, l'ouverture internationale de l'Ecole reste un objectif politique affiché dans les années 1990. On ne peut qu'être frappé, encore une fois, par la récurrence des solutions proposées. Depuis 1995, parallèlement à l'augmentation du nombre d'élèves étrangers à admettre par voie de concours, une nouvelle filière a été mise en place qui semble être une copie fidèle du statut d'auditeur libre, en raffinant la dénomination : aux côtés des CP1 (catégorie particulière 1$)^{10}$, élèves issus des classes préparatoires en France, au Maroc, en Tunisie ou au Liban, s'est créé un nouveau statut appelé CP2 (catégorie particulière 2) réservé aux étudiants d'autres pays n'ayant pas été formés dans un système d'enseignement proche de celui en vigueur en France : «... des jeunes gens et des jeunes filles qui pour certains d'entre eux n'avaient jamais entendu parler des possibilités de formation en France, qui ne savaient pas ce qu'est l'Ecole polytechnique et souvent qui $n^{\prime}$ avaient jamais quitté leur pays... ${ }^{11}$. Comme pour les auditeurs libres de jadis, un examen du dossier académique et des lettres de recommandations du candidat est effectué. Puis des épreuves orales permettent d'apprécier son niveau scientifique. Contrairement aux tentatives précédentes, les trois 
premières années de mise en œuvre de cette nouvelle mesure se sont soldées par l'arrivée de 12, puis 18 et enfin 24 élèves, la moitié étant originaire de l'Europe de l'Ouest et de I'Est, l'autre moitié d'A sie et d'A frique.

Les élèves de la CP2 n'obtiennent pas le diplôme d'ingénieur polytechnicien et par conséquent, doivent faire créditer leurs deux années d'étude auprès de leur université d'origine. Or, même si le caractère généraliste de l'enseignement reçu à l'Ecole polytechnique semble être apprécié, il correspond aux troisième et quatrième années d'études supérieures, là où les cycles intégrés de formation d'ingénieurs dans les pays étrangers dispensent des formations de spécialisation.

Une autre difficulté à l'attraction d'élèves étrangers à l'Ecole polytechnique réside dans le caractère payant des études. Malgrél'aide des ambassades, des industriels français et de la fondation de l'Ecole, seuls des programmes importants de bourses conséquentes du gouvernement français et de chaque pays peuvent venir à bout des frais de scolarité.

Il faudra donc se rendre à l'évidence : la Tunisie et le Maroc, puis le Maroc et la Tunisie sont restées, depuis le milieu des années 1950 jusqu'à nos jours, les plus grands viviers d'élèves étrangers de l'Ecole polytechnique, lui offrant pour la période 1986-2000, plus de $46 \%$ de ce contingent ( $26 \%$ pour les Marocains et $20 \%$ pour les Tunisiens), alors quel'Espagne, seul pays de l'Europe occidentale à dépasser le seuil de dix élèves pendant la même période, ne représente que $2,2 \%$ de l'ensemble $\mathrm{l}^{12}$.

Ces chiffres devront être rapprochés de la prédominance des étudiants marocains dans les grandes écoles françaises : en 1999, les Marocains, avec 1108 élèves, représentent le contingent le plus important, soit 8,5\% des 13144 étudiants étrangers que les grandes écoles françaises (ingénieurs et gestion) accueillent en formation diplômante et nondiplômante (formation continue et/ ou de courte durée) ; suivis des Algériens (814 étudiants) et des Tunisiens (573). Les étudiants marocains sont répartis selon un ratio de 1 pour 9 entre les écoles de gestion et les écoles d'ingénieurs ${ }^{13}$. A ce titre, le $M$ aroc se trouve dans une situation similaire à celle de l'Espagne (1 102 étudiants) à la seule différence que le ratio espagnol est de 3 pour 7 entre les écoles de gestion et les écoles d'ingénieur.

Toujours pour l'année 1999, le rapport du ministère des Affaires étrangères (note 13) français donne la répartition suivante pour les 150 étudiants marocains diplômés des Ecoles d'ingénieurs françaises, où l'on observe la place dominante de l'Ecole polytechnique en terme de flux annuel estimé, tal onnée par l'Ecole centrale et l'Ecole des ponts et chaussées ${ }^{14}$. 
De plus, selon la même source, près de 90 \% des élèves ingénieurs marocains bénéficient d'une bourse d'études, répartis dans un ratio de 6 pour 4 entre boursiers du gouvernement marocain et ceux du gouvernement français.

\begin{tabular}{|c|c|c|c|c|c|c|}
\hline Ecole & $\begin{array}{c}\text { D iplôme } \\
\text { d'ingénieur }\end{array}$ & D octorat & M aster & D EA & Total & Flux estimé \\
\hline $\begin{array}{c}\text { Ecole } \\
\text { polytechnique }\end{array}$ & $\mathbf{2 2}$ & $\mathbf{2}$ & $\mathbf{1}$ & $\mathbf{5}$ & $\mathbf{3 0}$ & $\mathbf{1 1}$ \\
\hline $\begin{array}{c}\text { Ecolecentrale } \\
\text { deParis }\end{array}$ & $\mathbf{2 5}$ & $\mathbf{4}$ & 3 & 1 & 33 & 8 \\
\hline $\begin{array}{c}\text { Ecoledesmines } \\
\text { deParis }\end{array}$ & $\mathbf{9}$ & 3 & 1 & 2 & 15 & 3 \\
\hline $\begin{array}{c}\text { Ecoledesponts } \\
\text { edhaussées }\end{array}$ & $\mathbf{2 1}$ & 3 & 3 & 5 & 32 & 7 \\
\hline $\begin{array}{c}\text { Ecoledes } \\
\text { técécoms }\end{array}$ & $\mathbf{9}$ & 6 & 3 & - & 18 & 3 \\
\hline ENSTA & $\mathbf{3}$ & - & - & - & 3 & 1 \\
\hline SUPELEC & 15 & 3 & - & - & 28 & 5 \\
\hline TOTAL & 104 & 31 & 11 & 13 & 159 & 38 \\
\hline
\end{tabular}

Source: Thierry Audric, Les étudiants marocains dans les écoles d'ingénieurs françaises, rapport dactylographié de la Sous-Direction de la coopération universitaire et scientifique, ministère des Affaires étrangères, mai 2000.

Sans doute faudra-t-il évaluer l'effet conjugué des programmes de coopération et de l'ouverture depuis le milieu des années 1980 de classes préparatoires au concours d'entrée des grandes écoles en Tunisie et au Maroc, mais aussi celui de la montée en puissance des écoles d'ingénieurs marocaines et tunisiennes pour expliquer l'évolution du nombre d'élèves originaires de ces deux pays dans les grandes écoles françaises et en particulier à l'Ecole polytechnique.

\section{Des ÉTUdiants MAghrébins À L'ÉCOLE POLYTECHNIQUe}

La période de l'entre-deux-guerres a donné à l'Ecole polytechnique les premiers élèves tunisiens et algériens ayant fréquentél'Ecole polytechnique régulièrement depuis. Leur admission à l'Ecole avant les indépendances revêt un caractère exceptionnel et dispersé dans le temps : deux A Igériens en 1919 et 1928 et un Tunisien en 1929.

Dans les pays du Maghreb, la politique éducative prônée par la France a souffert d'une grande instabilité. A la multiplicité des centres de décision se sont ajoutées des conceptions divergentes à propos des vertus de l'éducation que l'on offrirait aux « indigènes ». Une première conception, assimilationniste, attachée à l'idée de la France comme puissance coloniale humaniste et modernisatrice, a voulu faire des autochtones des sujets français par l'acquisition de la langue et de la culture françaises. Une seconde conception, prédominante auprès des Français des colonies, considérait le système 
d'éducation indigène comme une fabrique de déclassés et de rebelles nationalistes difficiles à administrer ${ }^{15}$.

L'originalité des parcours individuels des polytechniciens «coloniaux » se mesure aux nombreux obstacles qu'ils ont franchis avant d'arriver en France, mais aussi au choix d'une filière technique passant par l'Ecole polytechnique. En effet, même si les décrets de 1919 et 1920 stipulaient l'égalité des « indigènes » et des Français pour occuper des postes dans l'administration, tout en les excluant des fonctions d'autorité ; même si, plus tard, l'ordonnance du 7 mars 1944 a instauréleur égale admissibilitéà tous les emplois publics, le choix des filières débouchant sur des professions libérales (droit, médecine) est resté prépondérant dans les milieux étudiants issus des colonies ${ }^{16}$. Les débouchés pour les fonctions techniques étaient d'autant plus incertains que les patrons français des industries locales ne recrutaient que des Français ${ }^{17}$.

\section{En Algérie}

En Algérie où la France a opté depuis toujours pour l'assimilation culturelle, cette politique s'est accompagnée d'un isolement de l'élite de la masse « barbare et arriérée», pour reprendre la terminologie coloniale. Comme l'identification à la France était présentée comme la contrepartie del'ascension sociale par les études, beaucoup dejeunes algériens ont refusé d'abord d'entrer dans le jeu colonial pour enfin s'y prêter tout en s'engageant dans le mouvement étudiant anticolonial. La faiblesse numérique de la population dépassant le niveau d'instruction primaire est encore révélatrice : en 1910, on recense 25 diplômés indigènes, 20 étudiants qui se trouvent à Paris en 1928, contre 53 pendant l'année 1934-35, une centaine dix ans après, entre 200 et 250 en 1954. Certes, le mouvement s'est amplifié à partir de 1946, mais au début de la guerre d'indépendance, on ne compte toujours que 600 étudiants al gériens dans I'université française (200 à 250 à Paris, 90 à Montpellier, 70 à Toulouse, etc.). A la même époque, I'université d'Alger accueillait 5000 étudiants européens contre 400 al gériens seulement ${ }^{18}$. Le pourcentage d'étudiants musulmans al gériens oscille, en effet, entre 3 et $4 \%$ de 1915 à 1938 et entre de 7 et $8 \%$ de 1939 à $1961^{19}$.

En 1947-48, I'Algérie compte trois ou quatre ingénieurs diplômés des grandes écoles françaises, occupant des postes du rang de sous-préfet dans l'administration coloniale. Ce n'est qu'à partir de 1951-52 que les classes préparatoires algéroises commencent à recevoir des élèves indigènes en nombre important ${ }^{20}$. A vec le mot d'ordre de grève des études lancé par le Front de libération nationale (FLN), le rappel des 
étudiants et leur répartition dans d'autres pays, notamment en Europe de l'Est et au Moyen-Orient, le phénomène observé en amont ne se traduit pas non plus par une présence algérienne dans les grandes écoles. A près l'indépendance, la fermeture des classes préparatoires algéroises jusqu'en 1964, ainsi que l'absence de politique de coopération bilatérale dans ce domaine, seraient à l'origine de la faible représentation de I'Algérie dans le contingent important des élèves nord-africains del'Ecole polytechnique.

Pour ce qui est des A Igériens formés à l'Ecole polytechnique après l'indépendance (promotions 1963-1980), nous observons une propension aux études longues (DEA et doctorat) et une nette rupture quant au retour au pays : un seul cas de retour au pays (Sonatrach) parmi les élèves algériens des promotions 1963 à 1985 (14 situations professionnelles connues sur les 17 élèves recensés). Pour les 16 autres élèves des promotions 1986 à 1994, nos renseignements restent lacunaires puisque nous n'avons pu recenser que quatre situations professionnelles en France et aux Etats-Unis. La reconstitution des carrières devra donc se poursuivre.

\begin{tabular}{|c|c|c|c|c|c|c|c|c|}
\hline PAYS & $1921-39$ & $1944-54$ & $1955-64$ & $1965-69$ & $1970-74$ & $1975-79$ & $1980-85$ & TOTAL \\
\hline Algérie & 1 & - & 3 & 2 & 2 & 3 & 7 & 18 \\
\hline
\end{tabular}

La tendance à la faible représentation des Algériens dans le contingent des étudiants maghrébins del'Ecole polytechniquen'est pas corrigée de 1986 à 2000 où l'écart reste encore très important entre l'A Igérie d'une part, la Tunisie et le Maroc d'autre part, les élèves originaires de ces pays formant respectivement des contingents de 101 et 128 élèves sur la période considérée.

\begin{tabular}{|l|l|l|l|l|l|l|l|l|l|l|l|l|l|l|l|l|l|l|l|l|l|l|l|l|l|l|l|l|l|l|l|l|l|}
\hline PAYS & 1986 & 1987 & 1988 & 1989 & 1990 & 1991 & 1992 & 1993 & 1994 & 1995 & 1996 & 1997 & 1998 & 1999 & 2000 & TOTAL \\
\hline
\end{tabular} \begin{tabular}{|l|l|l|l|l|l|l|l|l|l|l|l|l|l|l|l|l}
\hline Algérie & 1 & 1 & 2 & 3 & 3 & 1 & & 1 & 4 & 1 & 1 & & 2 & 1 & 0 & 21 \\
\hline
\end{tabular}

\section{En Tunisie}

De 1921 à 1985, les élèves tunisiens ont constitué le plus grand contingent d'élèves étrangers, avec une présence régulière et constante dans les décennies 1960 et 1970 et une croissance exponentielle de 1980 à 1985 où leur nombre a été multiplié par trois.

\begin{tabular}{|c|c|c|c|c|c|c|c|c|}
\hline PAYS & $1921-39$ & $1944-54$ & $1955-64$ & $1965-69$ & $1970-74$ & $1975-79$ & $1980-85$ & TOTAL \\
\hline Tunisie & 1 & 2 & 9 & 10 & 13 & 12 & 33 & $\mathbf{8 0}$ \\
\hline
\end{tabular}


A près l'indépendance de la Tunisie en 1956, la présence des élèves tunisiens dans les grandes écoles techniques françaises et à l'Ecole polytechnique relève d'une politique délibérée de l'Etat tunisien et la création, en 1969, d'une filière spécifique (filière A de I'Ecole nationale d'ingénieurs de Tunis, préparant au concours des grandes écoles françaises).

Sur la période 1985-2000, la présence tunisienne s'est maintenue à un niveau élevé tout en connaissant une certaine baisse entre 1986 et 1990. Cette baisse pourrait s'expliquer, entre autres, par l'accord de transfert de technologies signé, au début des années quatre-vingt, entre la Tunisie et les Etats-Unis et qui a offert des bourses américaines à une sélection de bacheliers tunisiens pour se former dans les universités techniques américaines.

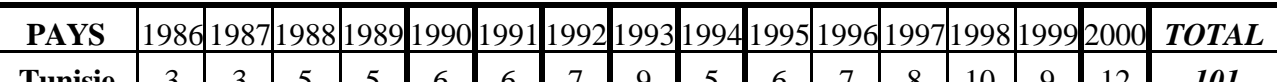

\section{Au Maroc}

La politique de la France vis-à-vis du Maroc s'inscrit quant à elle dès l'indépendance dans le contexte de la coopération et de la relève des cadres de la métropole. La France et le Maroc signent ainsi un traité de coopération, en 1958, ayant pour objectif d'aider l'ancienne colonie à se doter de l'encadrement administratif et technique qui lui manquait après le départ massif des cadres français. Une des clauses de ce traité engage la France à « ouvrir largement aux candidats présentés par le gouvernement marocain l'accès à tous les établissements français d'enseignement et d'application et à assurer, en cas de besoin, leur formation accélérée» ${ }^{21}$. En huit ans, 1500 étudiants marocains sont formés en France. En 1964-65, le ministère des Affaires étrangères accorde 550 bourses de coopération aux jeunes Marocains, dont 236 en région parisienne. Cette annéelà, on compte un élève admis à l'Ecole polytechnique 22 .

\begin{tabular}{|c|c|c|c|c|c|c|c|c|}
\hline PAYS & $1921-39$ & $1944-54$ & $1955-64$ & $1965-69$ & $1970-74$ & $1975-79$ & $1980-85$ & TOTAL \\
\hline Maroc & - & 1 & 8 & 8 & 9 & 13 & 30 & $\mathbf{6 9}$ \\
\hline
\end{tabular}

On peut supposer que les premiers élèves marocains de l'Ecole polytechnique ont aussi bénéficié des mesures de cette convention. Selon toute vraisemblance, les élèves signaient des engagements pluriannuels vis-à-vis de leur gouvernement. Au retour dans leur pays, la valeur de leur formation était probablement reconnue et des postes importants leur étaient proposés dans la haute fonction publique et dans les entreprises 
du même secteur. Ce qui expliquerait le retour au pays de la quasi-totalité des élèves marocains des promotions jusqu'en 1975, tandis qu'une très grande proportion des élèves des promotions, à partir de 1980, est restée en France ${ }^{23}$.

Au cours de ces quinze dernières années, le $M$ aroc a doubléle nombre de ses élèves à l'Ecole polytechnique par rapport aux trente premières années de son indépendance. Ainsi, nous sommes passés de 68 élèves de 1955 à 1985 à 128 entre 1986 et 2000 . Cette présence régulière et importante confère au Maroc le premier rang de la présence étrangère à l'Ecole polytechnique, devant la Tunisie, le Liban et le Viêt-nam²4.

\begin{tabular}{|c|c|c|c|c|c|c|c|c|c|c|c|c|c|c|c|c|}
\hline PAYS & 1986 & 1987 & 1988 & 1989 & 1990 & 1991 & 1992 & 1993 & 1994 & 1995 & 1996 & 1997 & 1998 & 1999 & 2000 & TOTAL \\
\hline Maroc & 6 & 4 & 9 & 7 & 5 & 14 & 8 & 9 & 5 & 7 & 6 & 11 & 11 & 17 & 9 & 128 \\
\hline
\end{tabular}

\section{Des ACTEURS DE L'HISTOIRE AUX ÉlITES « DE PEU 》}

Les premières générations des polytechniciens issus des colonies ou des protectorats ont regagné, en règle générale, leur pays d'origine, à la suite des années d'étudeen France. Bien que mouvementée, leur carrière professionnelle et publique tend à être caractérisée par trois étapes décisives : la première a été celle d'une intégration sociale difficile, la seconde est souvent marquée par un engagement politique de haut niveau et la troisième semble être celle d'un retour au cal me et à des carrières d'ingénieur plus traditionnelles. En effet, dès leur retour au pays d'origine, les polytechniciens de la première génération se retrouvent dans une situation professionnelle qui ne correspond pas aux compétences acquises en France. En Algérie notamment, la catégorie socioprofessionnelle des ingénieurs est pratiquement inexistantejusqu'à l'indépendance. Elley apparaît seulement dans les années 1970, lors des premières actions d'industrialisation, avec la création d'institutions de formation spécialisée ${ }^{25}$. En 1956, la Tunisie compte trente-six diplômés des grandes écoles françaises ${ }^{26}$. Contraints à occuper des postes subalternes dans l'administration et dans l'enseignement, la plupart de ces diplômés possèdent très peu d'expérience et n'ont exercé aucune fonction de responsabilité. Toutefois, des exceptions dont il convient de retracer la carrière personnelle existe : c'est le cas précisément d'E. Mouloud, élève français d'origine al gérienne de la promotion 1919, de S. Bouakouir, de la promotion 1927, diplômé de l'Ecole polytechnique et de l'Ecole du génie maritime.

\section{Les polytechniciens algériens}

Né en 1895 à Alger, Edouard Mouloud opte pour la citoyenneté française et vraisemblablement pour la religion chrétienne, du fait de son changement de prénom 
d'Omar à Edouard. Provenant de milieux populaires - son père est déclaré comme « boucher » à Alger dans le registre matricule -, il s'engage dans l'armée française au cours de la Première Guerre mondiale ${ }^{27}$. II réussit le concours de l'Ecole polytechnique à la fin de la guerre. Admissible dans le service public en 1921 dans l'artillerie métropolitaine (aéronautique), il grimpe, de 1935 à 1962, les échelons hiérarchiques dans l'administration des chemins de fer algériens. Sous-directeur de la Société nationale des chemins de fer algérienne (SNCFA) au moment de l'indépendance, il choisit d'y rester et de servir son pays d'origine, en occupant le poste de président du conseil d'administration de la SNCFA de 1964 jusqu'à sa mort en 1972.

Salah Bouakouir est, quant à lui, le premier haut fonctionnaire musulman servant en Algérie. Sa désignation à la direction de l'Energie, du Commerce et de I'Industrialisation du gouvernement général en 1947 constitue la première - et unique concrétisation des effets escomptés de l'ordonnance de mars $1944^{28}$. Néanmoins, sa promotion n'a pas dépassé, de toute évidence, le cas individuel. Cette nomination est le signe avant-coureur d'une ascension fulgurante : secrétaire adjoint des affaires économiques au gouvernement général en 1954, il fait partie, avec Claude Tixier, directeur général des Finances, d'un groupe de travail à l'origine d'un important plan intitulé « Perspectives décennales de développement économique de l'Algérie » (publié en mars 1958). A partir de 1957, il est directeur des Affaires économiques 29.

Après la nomination de Paul Delouvrier, nouveau délégué général du gouvernement en Algérie, S. Bouakouir entre dans son équipe en qualité de président du conseil supérieur du plan, créé à l'imitation du Plan d'équipement et de modernisation de la métropole. II y reste trois ans en fonctions jusqu'à sa mort, en septembre 1961, dans un accident dont les circonstances n'ont pas été élucidées. A I'indépendance, le FLN lui rend hommageen donnant son nom à un des plus grands boulevards d'Al ger jusqu'à une date récente où il a été «débaptisé ». Ce polytechnicien algérien, alors directeur de I'Equipement et de l'Industrie du gouvernement général, aurait constitué, en 1959-60, avec quelques autres de ses collègues une officine de renseignements pour le compte du FLN-GPRA (Gouvernement provisoire de la république al gérienne) ${ }^{30}$.

Mohammad Liassine serait le troisième et dernier élève algérien de l'Ecole polytechnique avant l'accession de ce pays à l'indépendance. Son arrivée à l'Ecole polytechnique en 1955 relève de la performance individuelle à l'instar de ses compatriotes E. Mouloud et S. Bouakouir. Issu du système d'enseignement indigène en Algérie, il fait partie des exceptions qui du fait de leurs brillants résultats scolaires, réussissent à intégrer 
I'enseignement réservé aux Français à partir du cycle secondaire. Son parcours scolaire a pu bénéficier de l'abolition du code de l'indigénat en 1947 qui perdure jusqu'en 1958. Devenu Français musulman du fait du nouveau statut de l'A lgérie, il dispose en théorie de droits identiques à ceux des citoyens français : il peut donc se présenter à l'Ecole polytechnique comme candidat français au concours général d'admission.

Dès son arrivée à Paris, un mot d'ordre de grève d'études est lancé par le FLN. II s'agit de la grève illimitée des cours et des examens déclenchée le 19 mai 1956 sous l'égide des associations d'étudiants et de l'UGEMA (Union générale des étudiants musulmans algériens). Cette grève relève alors d'un impératif catégorique, souligné dans la déclaration du comité directeur de I'UGEMA publiée à Paris. Aussi M. Liassine demandet-il la permission au FLN de continuer ses études pendant la grève. Ces dires sont confirmés par le responsable del'UGEMA del'époque, Belaïd Abdesselam, dont les souvenirs ont été publiés en $1990^{31}$.

A l'indépendance, M. Liassine et Ahmed Ghozali, ingénieur civil des Ponts et Chaussées, sont les seuls ingénieurs diplômés des grandes écoles françaises, dans les rangs de quel que 200 hauts fonctionnaires al gériens. Leurs carrières se déroulent dans le secteur industriel, aux côtés de Belaïd Abdelsselam, I'artisan du programme d'industrialisation algérien. Dans ses entretiens, B. Abdesselam relate comment I'Exécutif provisoire, mis en place au moment de l'accession à l'indépendance, a dû faire face à la remise en marche d'une machine économique grippée par le sabotage de l'OAS et par le départ des cadres français. Parmi les quelques cadres al gériens réunis à cet effet, il compte A. Ghozali qui venait de terminer ses études et $M$. Liassine qui avait terminé ses études avant l'indépendance et se trouvait affecté au sein des unités de l'A rmée de libération nationale $(A L N)$. Autorisé à rejoindre la délégation des affaires économiques, M. Liassine se voit confier la direction nouvellement créée de I'industrialisation. Au sein de cette direction, il a en charge la relance de la sidérurgie d'A nnaba et la création de la Société nationale de sidérurgie (SNS), dont il devient le premier directeur général ${ }^{32}$.

M. Liassine est bien un acteur de premier plan d'une industrialisation menée par la volonté politique de la fraction des «industrialistes » au pouvoir et financée par les revenus pétroliers. II réalise un projet pilote mobilisant 20000 travailleurs qui cristallise les attentes du gouvernement al gérien : dans les années 1950, la sidérurgie est consi dérée dans beaucoup de pays du Tiers-M onde comme la voie royale de l'industrialisation et du dével oppement. Legs de l'ancienne métropole qui continue à en parrainer la réalisation, 
la SNS accumule toutefois les conflits entre la maîtrise d'ouvrage (l'Algérie), les ingénieurs conseil (la France) et les entreprises soumissionnaires (la France et I'Union soviétique). Ici, le choix d'un polytechnicien algérien à la direction de l'entreprise semble avoir été dicté par la recherche d'un équilibre dans les rapports de forces entre le gouvernement al gérien et ses partenai res français. Cet équilibre est, en tout état de cause, largement en défaveur de l'Algérie, sur le plan technique.

A la fin des années 1970, à mi-parcours du programme d'industrialisation, des voix s'élèvent à l'encontre de M. Liassine au sein de l'équipe gouvernementale. II fait l'objet d'âpres critiques : endettement, effet de taille, investissements énormes sans création d'emplois à la hauteur, taux de fonctionnement très bas des unités industrielles, agriculture sacrifiée, etc. En 1978, le gigantesque ministère de l'Industrie est scindé en trois : I'ancien ministre Abdesselam conserve le secteur du transport, tandis que le portefeuille del'industrie lourde est confiéà M. Liassine et celui del'énergieà A. Ghozali. Ce n'est qu'en 1982, avec I'arrivée à la présidence de Chadli Ben-Djedid, que les adversaires de la politique menée jusqu'alors dans le domaine industriel obtiennent gain de cause. M. Liassine est écarté du pouvoir. II y est rappelé en 1991 dans le cabinet de crise formé par son camarade d'études et son compagnon de l'Industrie, A. Ghozali, qu'il suivra peu de temps après dans son départ pour la France.

\section{Les polytechniciens tunisiens}

La reconstitution de la carrière des élèves tunisiens des promotions 1929 à 1985 fait apparaître une première génération de polytechniciens entièrement vouée au service public ${ }^{33}$. Techniciens impliqués dans la construction du pays après l'indépendance, ils ont joué un rôle éminemment politique pendant les décennies 1960 et 1970, par l'étendue de leurs réalisations dans les secteurs privilégiés de l'intervention de l'Etat, à savoir les mines, l'énergie et les travaux publics.

Ils sont suivis par une deuxième génération de polytechniciens tunisiens tournéevers I'activité de bureaux d'études, au service du secteur public et parapublic, en partenariat avec des entreprises étrangères. Cette génération formée dans les années 1970 a su s'adapter à l'évolution de l'économie nationale, en prenant une part active dans la disparition des barrières douanières et l'ouverture du marché tunisien à la communauté européenne.

Une troisième génération (les années 1980) serait moins attachée au sentiment de devoir envers la patrie, parce qu'elle a été confrontée à un marché local de l'emploi plus difficile et à un climat politique moins libéral : ceux qui la représentent en Tunisie ont 
plutôt choisi d'assurer la gestion technique de la dette extérieure dans les banques. D'autres encore se sont installés en France où leurs carrières se confondent avec celle des polytechniciens français.

Les résultats partiels de notre enquête sur les promotions 1986-1995 confirment cette tendance au non-retour : sur les 47 élèves des promotions 1986 à 1994, nous avons connaissance de 32 situations professionnelles dont une seule se déroule au pays, à la Banque de Tunisie $e^{34}$.

\section{Les polytechniciens marocains}

La situation est légèrement différente au Maroc où il existe encore un «flux entrant » de diplômés de l'Ecole polytechnique française. Notre première analyse de la situation professionnelle des anciens élèves marocains est fondée sur les renseignements fournis dans l'annuaire 1990 : à cette date, les anciens élèves des promotions de 1948 à 1980, rentrés au Maroc, occupent des postes de directeur général et d'administrateur dans des entreprises publiques ou semi-publiques. Ceux issus des promotions 1981-1985 travaillent dans les mêmes entreprises. Ils gravissent les premiers échelons de cette hiérarchie. Le Maroc offre donc des situations suffisamment intéressantes aux diplômés formés en France et accorde de la valeur à leur qualification.

Le ministère des Travaux publics est le principal employeur des promotions de polytechniciens marocains jusqu'en 1970 : deux d'entre eux ont occupé le portefeuille de cette administration technique. Par la suite, c'est dans le groupe Omnium N ord A fricain (ONA) que l'on trouve la plus forte concentration de polytechniciens (7 entre 1972 et 1984). L'ONA est un immense holding qui comprend une centaine de sociétés dans les secteurs agro-alimentaire, mines, finances, immobilier, audiovisuel, automobile, textile,

etc. ${ }^{35}$ Les mines, le bâtiment, les travaux publics, les banques et l'industrie de montage sont par ordre décroissant les autres branches d'activité proposée aux anciens élèves de l'Ecole polytechnique.

Une autre analyse fondée sur l'Annuaire 1999, qui comprend les promotions de 1948 à $1994^{36}$, confirme une tendance plus poussée au non-retour. Elle révèle une différenciation plus importante du positionnement socioprofessionnel des polytechniciens marocains. De même, alors que pour les promotions de 1948 à 1980, le nombre de non-retour est relativement limité ( 6 sur les 33 carrières connues), il atteint un niveau plus important pour les promotions 1981 à 1985 (13 sur les 20 carrières connues). La tendance s'inverse de 1986 à 1994 (23 non retour sur 33 carrières connues). Les 
tableaux 1, 2 et 3 del'annexe 5 donnent un aperçu rapide des carrières embrassées par les ingénieurs polytechniciens de retour au Maroc.

La collecte d'informations sur les polytechniciens maghrébins devra être poursuivie dans les prochains mois par l'envoi d'un questionnaire et la réalisation d'entretiens avec un échantillon d'anciens élèves. L'objectif est de compléter les données prosopographiques ${ }^{37}$ avant de dresser quelques hypothèses sur le vivier et les modes de renouvellement des élites techniques en Tunisie et au Maroc, sur leur positionnement par rapport aux postes de commandement du secteur économique et sur la place que prend la formation à l'Ecole polytechnique française dans ce dispositif. 
Annexen 1

Répartition géographique des élèves étrangers 1921-1985

\begin{tabular}{|c|c|c|c|c|c|c|c|c|c|}
\hline & PAYS & $\begin{array}{c}\text { 1921- } \\
39\end{array}$ & $\begin{array}{c}1944- \\
54\end{array}$ & $\begin{array}{c}1955- \\
64\end{array}$ & $\begin{array}{c}1965- \\
69\end{array}$ & $\begin{array}{c}1970- \\
74\end{array}$ & $\begin{array}{c}1975- \\
79\end{array}$ & $\begin{array}{c}1980- \\
85\end{array}$ & TOTA \\
\hline 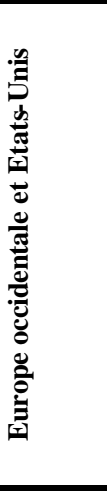 & $\begin{array}{l}\text { Espagne } \\
\text { Grèce } \\
\text { Luxembourg } \\
\text { Belgique } \\
\text { Etats-Unis } \\
\text { Italie } \\
\text { Pays-Bas } \\
\text { Angleterre } \\
\text { Monaco } \\
\text { Autriche } \\
\text { R.F.A. } \\
\text { Suisse } \\
\text { Norvège } \\
\text { Portugal } \\
\text { Suède } \\
\end{array}$ & $\begin{array}{l}3 \\
3 \\
2 \\
1 \\
1 \\
3\end{array}$ & $\begin{array}{l}1 \\
1 \\
1 \\
1 \\
1\end{array}$ & $\begin{array}{l}3 \\
5 \\
2 \\
1 \\
1 \\
1 \\
1 \\
1 \\
1\end{array}$ & $\begin{array}{l}1 \\
2 \\
1 \\
1\end{array}$ & $\begin{array}{l}3 \\
1 \\
1 \\
\\
1\end{array}$ & $\begin{array}{l}1 \\
1 \\
3 \\
3 \\
\\
3 \\
1 \\
1\end{array}$ & $\begin{array}{l}3 \\
1 \\
1 \\
1 \\
1\end{array}$ & $\begin{array}{c}11 \\
10 \\
7 \\
7 \\
6 \\
5 \\
4 \\
4 \\
4 \\
2 \\
2 \\
2 \\
1 \\
1 \\
1 \\
\end{array}$ \\
\hline 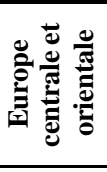 & \begin{tabular}{|l|} 
Pologne \\
URSS \\
Roumanie \\
Yougoslavie \\
Tchécoslovaquie \\
\end{tabular} & $\begin{array}{l}3 \\
1 \\
1 \\
1\end{array}$ & $\begin{array}{l}4 \\
1\end{array}$ & & & & 1 & 1 & $\begin{array}{l}4 \\
3 \\
3 \\
2 \\
1 \\
\end{array}$ \\
\hline 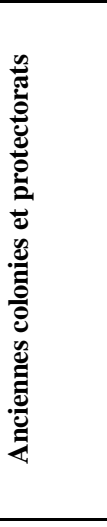 & \begin{tabular}{|l} 
Tunisie \\
Maroc \\
Liban \\
Viêt-nam \\
Algérie \\
Madagascar \\
Cameroun \\
Sénégal \\
Syrie \\
Cambodge \\
Côte d'Ivoire \\
Burkina Faso \\
Haiti \\
Laos \\
Togo \\
Ile Maurice \\
\end{tabular} & $\begin{array}{l}1 \\
2 \\
5 \\
1\end{array}$ & $\begin{array}{l}2 \\
1 \\
3 \\
2\end{array}$ & $\begin{array}{c}9 \\
8 \\
2 \\
24 \\
3 \\
1 \\
2\end{array}$ & $\begin{array}{l}10 \\
8 \\
4 \\
7 \\
2 \\
1 \\
1 \\
1\end{array}$ & $\begin{array}{c}13 \\
9 \\
8 \\
4 \\
2 \\
3 \\
2 \\
1 \\
1\end{array}$ & $\begin{array}{c}12 \\
13 \\
15 \\
5 \\
3 \\
2 \\
1 \\
2 \\
1\end{array}$ & $\begin{array}{l}33 \\
30 \\
18 \\
1 \\
7 \\
2 \\
2 \\
3 \\
1 \\
1 \\
1 \\
1 \\
1 \\
1\end{array}$ & $\begin{array}{c}80 \\
69 \\
52 \\
48 \\
18 \\
9 \\
8 \\
5 \\
4 \\
2 \\
1 \\
1 \\
1 \\
1 \\
1 \\
1 \\
\end{array}$ \\
\hline 总 & $\begin{array}{l}\text { Iran } \\
\text { Turquie } \\
\text { Egypte }\end{array}$ & $\begin{array}{l}8 \\
3 \\
3\end{array}$ & $\begin{array}{l}1 \\
1 \\
1\end{array}$ & $\begin{array}{l}1 \\
2 \\
4\end{array}$ & $\begin{array}{l}2 \\
1\end{array}$ & $\begin{array}{l}1 \\
1\end{array}$ & & $\begin{array}{l}4 \\
1 \\
1\end{array}$ & $\begin{array}{c}17 \\
9 \\
9\end{array}$ \\
\hline$\frac{.0}{4}$ & $\begin{array}{l}\text { Chine } \\
\text { Singapour } \\
\text { Indonésie } \\
\text { Taiwan }\end{array}$ & 4 & & & & & $\begin{array}{l}1 \\
1\end{array}$ & 10 & $\begin{array}{c}14 \\
1 \\
1 \\
1\end{array}$ \\
\hline \multirow{2}{*}{ 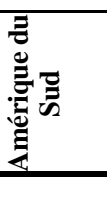 } & \begin{tabular}{|l|} 
Brésil \\
Chili \\
Uruguay
\end{tabular} & & & & 1 & & & $\begin{array}{l}1 \\
1\end{array}$ & $\begin{array}{l}1 \\
1 \\
1\end{array}$ \\
\hline & TOTAL & 46 & 21 & 73 & 43 & 51 & 72 & 130 & 436 \\
\hline
\end{tabular}


Annexe ${ }^{\circ} 2$

Tendance au retour dans le pays d'origine des élèves étrangers 1955-1985

\begin{tabular}{|c|c|c|}
\hline Total & 48 & \\
\hline Retour & 7 & $\begin{array}{l}\text { (dont } 2 \text { retours en France après } \\
1959)\end{array}$ \\
\hline France & 35 & \\
\hline Pays tiers & 1 & (Etats-Unis) \\
\hline Non identifiés & 5 & \\
\hline
\end{tabular}

Pour les promotions après 1952, aucun retour au pays.

\section{LIBAN}

\begin{tabular}{|c|c|c|}
\hline Total & 52 & \\
\hline Retour & 12 & \\
\hline France & 25 & \\
\hline Pays tiers & 5 & (4 Etats-Unis, 1 Canada) \\
\hline Non identifiés & 10 & \\
\hline
\end{tabular}

Pour les promotions après 1974 , aucun retour au pays.

\section{TUNISIE}

\begin{tabular}{|l|c|}
\hline Total & 80 \\
\hline Retour & 46 \\
\hline France & 27 \\
\hline Pays tiers & 7 \\
\hline non identifiés & - \\
\hline
\end{tabular}

Pour les promotions jusqu'en 1975, majorité de retour. Après 1975, intermittent avec majorité en France.

\section{MAROC}

\begin{tabular}{|l|c|}
\hline Total & 69 \\
\hline Retour & 36 \\
\hline France & 25 \\
\hline Pays tiers & 0 \\
\hline Non identifiés & 8 \\
\hline
\end{tabular}

Pour les promotions jusqu'en 1975, tous rentrés (exceptés 3 de confession israélite, naturalisés). Après 1980, très forte majorité en France.

\section{ALGERIE}

\begin{tabular}{|c|c|c|}
\hline Total & 18 & \\
\hline Retour & 6 & \\
\hline France & 10 & \\
\hline Pays tiers & 1 & (Sénégal pour l'ONU) \\
\hline Non identifiés & 1 & \\
\hline
\end{tabular}

Après 1977, pas de retour sauf un.

Source : Société amicale des anciens élèves de l'Ecole polytechnique, Annuaire, 1990. 
Annexen ${ }^{\circ} 3$

Répartition géographique des élèves étrangers 1986-2000

\begin{tabular}{|c|c|c|c|c|c|c|c|c|c|c|c|c|c|c|c|c|}
\hline PAYS & 1986 & 1987 & 1988 & 1989 & 1990 & 1991 & 1992 & 1993 & 1994 & 1995 & 1996 & 1997 & 1998 & 1999 & 2000 & OTAL \\
\hline Maroc & 6 & 4 & 9 & 7 & 5 & 14 & 8 & 9 & 5 & 7 & 6 & 11 & 11 & 17 & 9 & 128 \\
\hline Tunisie & 3 & 3 & 5 & 5 & 6 & 6 & 7 & 9 & 5 & 6 & 7 & 8 & 10 & 9 & 12 & 101 \\
\hline Liban & 3 & 2 & 2 & 0 & 7 & 3 & 2 & 3 & 2 & 3 & 1 & & 1 & 1 & 3 & 33 \\
\hline Viêt-nam & & & & & 1 & & & 1 & & & 1 & 2 & 4 & 4 & 11 & 24 \\
\hline Russie & & & & & & & 1 & & & & 1 & 1 & 4 & 6 & 9 & 22 \\
\hline Algérie & 1 & 1 & 2 & 3 & 3 & 1 & & 1 & 4 & 1 & 1 & & 2 & 1 & 0 & 21 \\
\hline $\begin{array}{l}\text { Roumanie } \\
\text { Chine }\end{array}$ & & & & & & & & & & & 4 & 3 & 6 & $\begin{array}{l}6 \\
4 \\
\end{array}$ & $\begin{array}{l}5 \\
6 \\
\end{array}$ & $\begin{array}{l}18 \\
16 \\
\end{array}$ \\
\hline $\begin{array}{l}\text { Iran } \\
\text { Sénégal }\end{array}$ & 4 & & & 2 & 1 & 1 & & 1 & 1 & 2 & 1 & 1 & 4 & $\begin{array}{l}2 \\
2 \\
\end{array}$ & $\begin{array}{l}4 \\
1 \\
\end{array}$ & $\begin{array}{l}14 \\
13 \\
\end{array}$ \\
\hline $\begin{array}{l}\text { Espagne } \\
\text { TOTAL* } \\
\text { Elèves } \\
\text { étrangers }\end{array}$ & 21 & 14 & 22 & 17 & 28 & $\begin{array}{c}1 \\
29\end{array}$ & 18 & 32 & 22 & $\begin{array}{c}1 \\
24\end{array}$ & $\begin{array}{c}1 \\
30\end{array}$ & $\begin{array}{c}1 \\
32\end{array}$ & $\begin{array}{c}4 \\
61\end{array}$ & $\begin{array}{c}2 \\
65\end{array}$ & $\begin{array}{c}1 \\
78\end{array}$ & $\begin{array}{c}11 \\
493\end{array}$ \\
\hline $\begin{array}{l}\text { TOTAL } \\
\text { Elèves } \\
\text { français }\end{array}$ & 336 & 313 & 309 & 340 & 360 & 380 & 400 & 399 & 400 & 400 & 400 & 398 & 400 & 400 & 400 & 5635 \\
\hline
\end{tabular}

*Ce total comprend des ressortissants des pays énumérés ci-dessous, leur nombre ne dépassant pas dix par pays sur la période considérée :

Allemagne, Autriche, Andorre, Belgique, Bénin, Bulgarie, Cameroun, Canada, Chine-Taiwan, Colombie, Corée, Côte d'Ivoire, Croatie, Danemark, Equateur, Grande Bretagne, Grèce, Hollande, Hongrie, Indonésie, Italie, Japon, Luxembourg, Lettonie, Madagascar, Maurice, Monaco, Mexique, Norvège, Pologne, Suède, Syrie. 
Annexe ${ }^{\circ} 4$

G rille des notices biographiques

- NOM

- PRENOM

- $\mathrm{H} / \mathrm{F}$

- PROMOTION

- RELIGION

- BOURSIER

- AMONT 1 (Lycée)

- AMONT 2 (Classe préparatoire)

- AVAL 1 (Ecole d'application)

- AVAL 2 (Formation complémentaire, DEA, Doctorat)

- ACTIF ANNUAIRE 1990 (Activité professionnelle, A nnuaire 1990)

- ACTIF AN NUAIRE 1997 (Activité professionnelle, A nnuaire 1997)

- ACTIF ANNUAIRE 1999 (Activité professionnelle, A nnuaire 1999)

- ACTIF 3 ANS (Activité 3 ans après le dernier diplôme)

- ACTIF 5 ANS

- ACTIF 10ANS

- ACTIF 15 ANS

- ACTIF 20 ANS

- ACTIF FINAL

- ADRESSE 


\section{Annexen ${ }^{\circ} 5$}

Tableau 1. Situation professionnelle des polytechniciens marocains en 1999 Promotions 1948-1980

\begin{tabular}{|lr|}
\hline $\begin{array}{l}\text { Ministres, anciens ministres, directeurs département ministériel } \\
\text { (Travaux publics, Commerce, Finances) }\end{array}$ & $\mathbf{8}$ \\
\hline Gouverneur Préfecture & $\mathbf{1}$ \\
\hline Présidents, directeurs d'entreprises publiques (RAM, ONE ...) & $\mathbf{3}$ \\
PDG entreprises industrielles et de conseil & $\mathbf{6}$ \\
\hline Directeurs ONA & $\mathbf{3}$ \\
\hline Directeur Banque & $\mathbf{1}$ \\
Consultants / Conseil & 2 \\
\hline Banque & 1 \\
\hline OCP & 1 \\
Enseignant universitaire & 1 \\
\hline Carrières en France et dans d'autres pays* & $\mathbf{6}$ \\
\hline Carrières inconnues & 7 \\
TOTAL & $\mathbf{4 0}$ \\
\hline
\end{tabular}

* (dont 3 élèves israélites d' origine marocaine)

Tableau 2. Situation professionnelle des polytechniciens marocains en 1999 Promotions 1981-1985

\begin{tabular}{|lr|}
\hline Directeurs entreprises industrielles & 2 \\
\hline Chefs de division ministérielle & 1 \\
Directeur ODEP & 1 \\
\hline Banque & 1 \\
\hline ONA & 1 \\
Enseignant universitaire & 1 \\
\hline Carrières en France et dans d'autres & $\mathbf{1 3}$ \\
pays & \\
\hline Carrières inconnues & 7 \\
TOTAL* & $\mathbf{2 7}$ \\
\hline
\end{tabular}

* D ont 1 fille. 


\section{Tableau 3. Situation professionnelle des polytechniciens marocains en 1999} Promotions 1986-2000

\begin{tabular}{|l|r|}
\hline $\begin{array}{l}\text { Entreprises industrielles } \\
\text { (Industries Maroc modernes, Hamel, Procter \& Gamble, ONA) }\end{array}$ & 4 \\
\hline Ministères (Pêches maritimes, Equipement, Travaux publics) & 3 \\
\hline Entreprises publiques (RAM, ODEP) & 2 \\
Enseignant universitaire & 1 \\
\hline Carrières en France et dans d'autres pays & $\mathbf{2 3}$ \\
\hline Carrières inconnues* & 34 \\
TOTAL** & $\mathbf{6 7}$ \\
\hline
\end{tabular}

\footnotetext{
* Etant donné le nombre important d'élèves pour lesquels l'Annuaire de 1999 ne fournit pas de renseignement sur la carrière, les chiffres de ce tableau doivent être interprétés avec précaution en attendant la fin de la collecte d'informations.

** Dont 3 filles.
}

\section{N OTES}

${ }^{1}$ Voir annexe $n^{\circ} 1$

${ }^{2}$ La question de «l'augmentation du nombre des élèves étrangers, notamment européens » est mise à l'ordre du jour du conseil de l'Ecole le 07/ 10/ 1968 : Cote : X2c29, t.19, p. 89-90. Pour attirer les étrangers d'un certain niveau, le groupe de travail chargé de réformer l'institution propose, en 1974, six recommandations. Registre des procès-verbaux du conseil de perfectionnement de l'Ecole polytechnique. Archives E.P. : Cote : X2c29, t.20, p.120.

3 Délibérations du 26/11/ 1959. Ibid. : Cote: X2c29, t.17, p. 245.

4 Ibid., t.18, p. 202.

5 Un seul candidat en trois ans qui s'est finalement désisté lbid., t. 19, pp. 89-90.

6 Registre des procès-verbaux du conseil d'administration de l'Ecole polytechnique. Archives E.P. : Cote: $X 2$ c29, t. 20, p. 120 .

7 Ibid., p. 171.

8 Un an plus tard, le conseil redonne aux épreuves de langues vivantes leur caractère facultatif pour les étrangers. Ibid., p. 384

${ }^{9}$ Voir annexe ${ }^{\circ} 2$

10 Les élèves étrangers de l'Ecole polytechnique sont également connus sous le nom générique de « catégorie particulière ».

${ }^{11}$ SENEOR R. (directeur des Relations extérieures del'Ecole polytechnique), « L'internationalisation de l'Ecole », La Jaune et la Rouge. Revue mensuelle de la Société amicale des anciens élèves de l'Ecole polytechnique, novembre 2000, n 559, pp. 13-19.

12 Voir annexe $n^{\circ} 3$.

13 AUDRIC Thierry, Les étudiants marocains dans les écoles d'ingénieurs françaises, rapport dactylographié de la Sous-Direction de la coopération universitaire et scientifique, ministère des Affaires étrangères, mai 2000. Ce rapport est fondé, entre autres, sur l'enquête menée par la Conférence des grandes écoles en février 1999 sur le nombre des étudiants étrangers dans les écoles d'ingénieurs et de gestion. Nous remercions $\mathrm{E}$. Gobe de nous l'avoir communiqué.

14 Ibid., tableau 3, p. 4.

15 MARSEILLE J., Empire colonial et capital isme français. H istoire d'un divorce, Paris, Albin Michel, 1984, p. 240 et suite 

pp. 39-46.

16 PERVILLÉ G., Les étudiants al gériens de I'U niversité française 1880-1962, Paris, Ed. du CNRS, 1984,

17 BARJOT D., «Les entreprises de travaux publics face à la décolonisation : une adaptation difficile, mais réussie? (1940-1956) », in AGERON C.-R. (dir.), Les Chemins de la décolonisation de l'Empire colonial français, Paris, Ed. du CNRS, 1986, pp. 157-164.

18 STORA B., Ils venaient d'A Igérie. L'immigration algérienne en France (1912-1992), Paris, Fayard, 1992, p. 239. G. Pervillé avance le chiffre de 1200 étudiants al gériens en 1954, dont 600 à l'Université d'Alger.

19 Ibid.

20 De 1925 à 1962, le lycée Bugeaud d'A Iger a comporté une classe de mathématiques supérieures et une classe de mathématiques spéciales, appelée «taupe arabe ». Malgré son appellation, elle n'a compté qu'un nombre extrêmement faible d'élèves de confession musulmane.

${ }^{21}$ THEYSSET E., «Huit années de coopération technique avec le Maroc», Revue juridique et politique, Paris, septembre 1965, pp. 481-495.

22 Ibid.

${ }^{23}$ Voir annexe $n^{\circ} 2$.

${ }^{24}$ Voir annexe $n^{\circ} 3$.

25 AKKACHE A., «Ingénieurs et emploi. Quelques données sur l'Algérie », in LONGUENESSE E. (dir.), Bâtisseurs et bureaucrates. Ingénieurs et société au M aghreb et au M oyen-O rient, Lyon, Maison de l'Orient méditerranéen, 1990, pp. 147-154, p. 147.

26 La ventilation est la suivante: 5 diplômés del'Ecolepolytechnique, 10 del'Ecoledes mines de Paris et de Saint-Etienne, 4 de l'Ecole de l'hydraulique de Grenoble, 3 de l'Ecole des télécommunications, 2 de I'Ecole des travaux publics, 1 de l'Ecole centrale de Paris. BENSALEM L., «La Profession d'ingénieur en Tunisie. Approche historique », in LONGUENESSE E. (dir.), op. cit., pp. 81-93, p. 91.

27 Les registres-matricules contiennent sous forme manuscrite des informations d'état civil, le signalement physique de l'élève, son centre d'examen, son rang d'entrée et de sortie et la carrière choisie à I'issue de l'Ecole polytechnique. Ils sont gardés aux Archives de l'Ecole sous la cote X2C/ C.

28 PERVILLÉ G., op.cit., p. 41.

29 nformations recueillies dans la notice nécrologique consacrée à S. Bouakouir, La Jaune et la Rouge, 1961, p. 51 ; complétées auprès de M. C.-R. A geron. N ous lui présentons nos plus vifs remerciements.

30 HARBI M., Le F.L.N., mirage et réalité Des origines à la prise du pouvoir (1945-1962), Paris, Jeune Afrique, 1980, p. 281. D'après M. C.-R. Ageron, Paul Delouvrier aurait utilisé S. Bouakouir dans des négociations secrètes avec le FLN concernant le libre acheminement du pétrole saharien, moyennant dédommagement financier de la part des compagnies pétrolières.

31 BENNOUNE M., EL-KENZ A., Le hasard et I'histoire. Entretiens avec Belaid A bdesselam, t. 1, ENAG éditions, 1990, p. 120

32 Ibid. , p. 193

${ }^{33}$ Notre étude plus détaillée des polytechniciens tunisiens a fait l'objet d'une contribution à l'ouvrage consacré aux trajectoires sociales et itinérai res migratoires des diplômés maghrébins, publié sous la direction de Vincent Geisser. Nous en reprenons, ici, les lignes essentielles. Cf GEISSER V. (dir.), D iplômés maghrébins d'ici et d'ailleurs. Trajectoires sociales et itinéraires migratoires, Ed. CNRS, 2000, pp. 179-192.

34 II faudra sans doute corriger ces données par la collecte d'informations sur les 15 situations professionnelles ne figurant pas à ce jour dans l'Annuaire des anciens élèves qui dispose de moyens autrement plus efficaces pour repérer les polytechniciens dans les entreprises et organismes français.

35 Groupe ONA , «Rapport annuel », 1989.

36 Les promotions 1995 et suivantes n'ayant pas encore terminé leurs études, l'A nnuaire de 1999 ne fournit pas de renseignements professionnels sur eux.

37 Pour la grille des notices biographiques, voir annexe $n^{\circ} 4$. 


\title{
L'Ecole polytechnique d'Alger : la formation inachevée d'une élite technique
}

\author{
Mohamed BEN G UER N A
}

De quelle manière et dans quelle mesure l'Ecole polytechnique d'Alger a-t-elle participé à la production d'une élite technique ? Peut-on émettre l'hypothèse que cette « grande école » al gérienne, créée en 1962 sur le modèle académique français, a constitué un foyer de formation autorisant la reproduction d'une élite sociale, dans les huit années qui ont suivi l'indépendance du pays ? En effet, en 1970, les organismes internationaux, tels que le PNUD et I'UNESCO, cessent d'apporter un soutien pédagogique à l'école. L'établissement se redéploie en ne comptant que sur ses moyens matériels et humains.

Les responsables politiques des premiers temps de l'indépendance ne se sont pas clairement exprimés sur cette notion d'élite technique. Mais leurs visées élitistes et leurs attentes peuvent se percevoir si l'on analyse les programmes de formation mis en place, et si I'on retrace les itinéraires scientifiques et professionnels des premiers ingénieurs polytechniciens. Ainsi, dans une première partie, nous étudierons le projet qui se caractérise par une approche pédagogique mais aussi politique. Nous verrons comment le choix du corps enseignant et des programmes ont été conçus en référence à des normes et des exigences internationales. Puis nous étudierons la mise en œuvre pédagogique proprement dite, les premiers temps de la formation. Nous verrons comment les responsables se sont doté des meilleures conditions de réalisation, pour la sélection des élèves, pour les modalités d'enseignements et pour l'évaluation des niveaux de formation. Cette option a favorisé l'insertion professionnelle des premières promotions d'ingénieurs. Par la suite, cependant, se multiplient les obstacles et les contraintes. C'est durant cette phase dite de «reproduction », marquée par un relatif désengagement politique, que le projet pédagogique a été compromis. 


\section{LE TEM PS DU PROJET}

L'Institut industriel d'Algérie, créé en 1925, proposait dès l'année suivante une formation de techniciens supérieurs ayant vocation à travailler au sein d'entreprises et dans le secteur des travaux publics. En dépit d'une interruption liée à la Seconde Guerre mondiale, cet Institut est maintenu en activité durant près de trente ans. Dès 1945, sous la nouvelle dénomination d'École nationale d'ingénieurs des travaux publics et du bâtiment (ENITPB), I'ancien établissement se reformule par un rattachement au secrétariat d'Etat à l'Enseignement technique et, surtout, par la création d'un diplôme d'ingénieur. Son action pédagogique est encore élargie dix ans plus tard, en 1955, avec la création de deux autres spécialités, l'électronique et l'électrotechnique. Ce n'est qu'en 1958 qu'il prend le nom d'École nationale des ingénieurs algériens (ENIA). Celle-ci formait des ingénieurs dans quatre spécialités : les travaux publics, le bâtiment, I'électronique et la mécanique. Or, en 1962, l'ENIA est fermée: I'ensemble des personnels technique et administratif, de même que le corps enseignant et les étudiants, sont contraints de quitter l'établissement. Pour maintenir la formation, la direction transfère ses activités d'enseignement en France : jusqu'en 1965, les anciens étudiants de l'ENIA ont pu y achever leurs études et obtenir un diplôme dans des écoles d'ingénieurs, à Strasbourg ou à Paris (Clichy).

Quelques mois après l'indépendance, le gouvernement algérien engage toutefois les procédures nécessaires à la réouverture d'une grande école dans le pays ; et ils choisissent de l'appeler l'École nationale polytechnique. II s'agit bien d'un projet politique qui accorde une place primordiale à la formation des cadres techniques. Les propos de l'ancien dirigeant Houari Boumedienne sont clairs à ce sujet :

\footnotetext{
«La politique du pouvoir révolutionnaire repose sur la formation des cadres algériens en nombre et en valeur nécessaire. Tant que nous n'aurons pas résolu ce problème, notre pays connaîtra toujours une situation que nous n'acceptons pas. $\gg^{1}$
}

Le gouvernement algérien cautionne donc un grand projet éducatif dans laquelle l'École polytechnique occupe une place centrale. Pour Ahmed Taleb Ibrahimi, alors le ministre de l'Éducation, cette école symboliserait, à la fois, «l'une des orientations fondamentales de notre enseignement, l'orientation scientifique et technique ; « «'une de nos grandes options dans la grande voie du développement, l'expansion économique et industrielle du pays »; enfin, «l'une des constantes de notre politique: la coopération et la compréhension internationale $»^{2}$. La mobilisation des responsables pédagogiques et des premiers élèves ingénieurs vient renforcer cette volonté politique. On va jusqu'à puiser dans le vivier des militants du FLN pour assurer le démarrage de l'institution. Le 
texte du télégramme envoyé par l'un des artisans du projet, à ses étudiants éparpillés dans le pays et à l'étranger, exprime ses attentes : «Je souhaite créer une école al gérienne d'ingénieur de très haut niveau, il me faut une première promotion ; accepterez-vous de merejoindre? Démarrage en novembre $1962 \gg 3$.

On soulignera que le projet de réouverture de cette grande école d'ingénieurs (dans sa triple dimension, politique, idéologique et pédagogique) affiche l'objectif, bien identifié cette fois, de former une élite technique. Mais l'engagement commun des décideurs, des animateurs et des bénéficiaires du projet suppose, en premier lieu, sa traduction en termes pédagogiques. Pour ce faire, les autorités politiques s'adressent aux N ations Unies et à I'U nesco. Dans une requête déposée durant I'hiver 1962 pour solliciter un soutien financier, les signataires expriment le souhait que « la formation puisse être organisée dans les domaines suivants : mécanique, transport et mécanisation agricole ; génie civil (bâtiment, travaux publics et hydraulique), électricité (électrotechnique et électronique) ; géologie appliquée» ${ }^{4}$. A u printemps 1963, la direction du PNUD propose, en priorité, la réouverture des deux départements existant antérieurement au sein del'exENIA. Un premier accord est ainsi signé le 15 novembre de la même année. Parallèlement, les discussions sont engagées pour créer de nouvelles spécialités. Ce second projet ${ }^{5}$ précipite l'ouverture de trois autres départements, au cours de l'année universitaire 1963-1964 : ceux de mécanique, de génie et chimie, de pétrochimie et d'économie appliquée à l'industrie. Un enseignement en sciences fondamentales est commun aux élèves detous les départements et un enseignement spécialisé est assurépar chacun des départements. Par ailleurs, diverses procédures administratives et juridiques sont mises en places pour faciliter l'aide des organismes internationaux : accompagnement pédagogique et soutien matériel ont été étalés sur cinq ans. Enfin, des bourses sont attribuées par la coopération française qui suit en cela les recommandations du PNUD et de I'UNESCO. Toutefois, quelle est l'ossature de la grande école nationale d'Algérie, dans la décennie 1960 ? Qui compose le corps enseignant ? Et à qui est-elle dispensée ? Quels sont les contenus de la formation ?

\section{LE TEMPS DE LA FORMATION}

Dès 1963, près d'une quarantained'enseignants al gériens de différents grades (dont 14 professeurs), ainsi qu'une cinquantaine de coopérants français, pour la plupart maîtres assistants, constituent le corps enseignant, avec dix experts du PNUD et de I'UNESCO. Les activités pédagogiques sont assurées, pour moitié, par des enseignants français, 
engagés dans une politique de la coopération culturelle bilatérale. Certains d'entre eux ayant été recrutés auparavant, pour enseigner dans I'ancienne ENSIA, n'ont pas quitté I'Algérie en juin 1962. L'encadrement professoral repose également sur les compétences des ingénieurs algériens, diplômés d'avant l'indépendance, ou sur des universitaires et scientifiques algériens appartenant aux réseaux de soutien au Front de libération nationale (FLN). Par la suite, ce premier corps enseignant devait être renforcé, grâce à l'assistance des organismes internationaux (cf. le tableau à la fin du texte).

Les études dispensées à l'Ecole polytechnique d'Alger, sur quatre ans, sont sanctionnées par un diplôme d'ingénieurs équivalant à ceux des grandes écoles étrangères. A l'origine, deux filières règlent le recrutement des polytechniciens. La première concerne de jeunes A Igériens qui ont terminé des études secondaires, avec un baccalauréat de mathématiques. La seconde autorise à se présenter les élèves qui, ayant entamé un cycle secondaire, n'ont pas pu l'achever en raison de la conjoncture de guerre. Dans les deux cas, cette population étudiante doit réussir un examen d'entrée.

Au cours de l'année 1968, cependant, la durée des études est prolongée d'une année. Cette formation en cinq années entraîne la suppression de l'examen d'entrée et un allongement de la période de tronc commun qui se fait dorénavant en trois ans. Pour les experts, une telle décision est motivée par le fait que l'année supplémentaire doit être « consacrée, en grande partie, aux sciences de base (mathématiques, physique, chimie et sciences sociales) ». Elle doit en principe faciliter « le travail des étudiants, en donnant la possibilité de combler les lacunes éventuelles de l'enseignement secondaire »6.

A la fin de l'année 1969, s'amorce la réforme du système éducatif algérien qui consacre le principe de la démocratisation de l'enseignement : I'abolition de l'examen d'entrée a servi d'emblème au nouveau dispositif. II nous semble intéressant de souligner que l'évolution puise sa légitimité dans un travail de comparaison entre les différents systèmes de formation dont sont originaires les experts internationaux, en posteà l'Ecole polytechnique d'Alger. L'année universitaire 1969-1970 est aussi marquée par la fin du programme d'accompagnement des organismes internationaux, et par un nouveau contexte politique.

Une autre modification importantes'inspire d'un courant pédagogique qui accorde une place primordiale à la confrontation de l'élève ingénieur avec les réalités professionnelles. Les partisans du projet ne manquent pas de souligner que «l'équipement des laboratoires des divers départements de l'école est conforme à ce qu'il 
est attendu d'une grande école d'ingénieurs », et que la bibliothèque de l'école peut être considérée comme satisfaisante ${ }^{7}$. II apparaît enfin que cette réforme de la formation d'ingénieurs tend à développer des valeurs humanistes : celles-ci se matérialisent par une activité associative et la publication d'un bulletin, au sein de l'établissement.

En somme, les élèves de Polytechnique prennent conscience que l'enjeu est de taille : outre une formation d'excellence, ils doivent assurer la relève d'une élite technique. Quelles options l'École polytechnique choisit-elle ? Comment réagit-elle face aux transformations politiques ? Et quels itinéraires empruntent les premières promotions de polytechniciens al gériens, ceux-là même qui cherchent à promouvoir des instruments garantissant le développement de leur profession ?

\title{
LE TEMPS DE LA REPRODUCTION
}

Plusieurs paramètres viennent d'orienter la stratégie de formation et de reproduction d'une élite technique en Algérie. En premier lieu, nous l'avons vu, il est décidé de supprimer le concours d'entrée à Polytechnique. Certains experts internationaux n'hésitent pas, par des observations, à attirer l'attention sur le fait que cette mesure tend à abaisser le niveau des postulants :

\begin{abstract}
«La suppression de l'examen d'entrée à permis d'attirer à l'école un nombre suffisant de candidats ; toutefois, elle a fait affluer des étudiants ayant accompli des études secondaires de typetrès divers, qui neles prépar ent que de manière souvent imparfaiteà suivre le programme de l'école. En particulier, les étudiants en provenance de l'enseignement technique, s'ils présentent de solides connaissances dans les disciplines technologiques, manifestent certaines faiblesses dans le domaine des sciences exactes et, par suite, seront sérieusement handicapés au début de leurs études d'ingénieurs. »8
\end{abstract}

Au vu de la structure socio-économique de la société algérienne en 1970, en particulier de l'origine rurale d'une majorité de candidats, on voit effectivement naître des difficultés d'intégration au rythme du travail de l'École.

En deuxième lieu, le projet pédagogique de Polytechnique accorde une place importante à la formation de cadres enseignants, par un système de bourse à l'étranger. Le but est de garantir l'entière relève des étrangers par un corps enseignant de nationalité algérienne. Mais cette stratégie de bourses à l'étranger ne produit pas le résultat escompté. Lorsqu'ils reviennent en Algérie, les anciens boursiers sont tentés d'accepter des postes de responsabilité dans l'industrie. Ces ingénieurs polytechniciens n'ont en effet aucune peine à s'insérer dans les différents secteurs de l'économie. 
Un troisième paramètre favorise cette tendance : une politique de développement fondée sur l'industrialisation du pays est mise en œuvre, coïncidant avec le départ massif des cadres techniques français. Aussi, les entreprises publiques naissantes proposentelles des compléments financiers importants aux ingénieurs diplômés, quand elles n'offrent pas à des élèves ingénieurs la possibilité de poursuivre des études à l'étranger, pour s'assurer, àl'avenir, deleurs services. Lefaible potentiel techniquedel'Algérie postindépendante ayant conféré une place centrale à leur École, les polytechniciens al gériens sont sollicités en permanence par les entreprises industrielles qui recherchent la qualité de leur formation. En position de force sur le marché de travail, ces mêmes polytechniciens se trouvent propulsés à des postes de gestionnaires et de cadres administratifs.

Ceglissement de la fonction productive del'ingénieur vers la sphère administrative va constituer la première contrainte du processus de reproduction. Conscients de ce phénomène, certains polytechniciens mettent en place une association d'anciens élèves. Ils veulent ainsi mettre à profit leurs positions d'autorité dans l'entreprise et dans l'administration pour garantir et préserver l'emploi des élèves ingénieurs.

\section{CONCLUSION}

Le tableau que nous avons brossé à travers l'exemple de l'Ecole polytechnique d'Alger, des différentes étapes de la formation d'excellence, dans le domaine scientifique et technique, illustre la complexité des mécanismes de reproduction d'un modèle académique, et nous questionne sur les conditions du transfert des expériences de formation. Dans le cadre de nos travaux, il ne s'agit que d'une piste de recherche parmi d'autres. Néanmoins je souhaiterais livrer à la discussion deux types de réflexions. D'une part, dans quelle mesure et selon quelles modalités, un système de formation d'élite, reposant sur des garanties scientifiques et pédagogiques, peut-il résister aux contingences politiques et assurer sa pérennité ? D'autre part, la complexité des réalités culturelles ne rend-elle pas opportune une réflexion sur les fondements pédagogiques et philosophiques des modèles de formation des ingénieurs ? Les sociétés maghrébines ont su capitaliser des expériences riches et variées, en ce domaine. Cependant, les transformations socio-économiques actuelles des pays du Maghreb nous imposent d'engager un débat, peut-être plus théorique, sur les nouvelles perspectives de formation des cadres techniques. 
Tableau. Composante du corps enseignant de l'Ecole polytechnique d'Alger en 1963

\begin{tabular}{l|c|c|c|}
\hline Origine & Algériens & Experts UNESCO & Coopérants \\
$\begin{array}{l}\text { Professeurs ou maîtres } \\
\text { de conférence }\end{array}$ & 2 & 9 & 5 \\
Maîtres Assistants & 14 & 1 & 11 \\
Assistants & 9 & - & 30 \\
Chargés de cours & 12 & - & 7 \\
TOTAL & 37 & 10 & 53 \\
\hline
\end{tabular}

N OTES

1 H. Boumedienne, El M oudjahid, 9 juillet 1969.

2 TALEB IBRAHIMI A hmed, D ela décolonisation à la révolution culturelle, 1967, 1972, Ed. Sned, Alger, 1973.

3 Document interne de l'association des ingénieurs, 1970.

4 Document : Requête pour l'ouverture de l'école, 1962, PNUD, ALG1.

5 Document UNESCO, 1969.

6 Ibidem.

7 Ibid. 



\title{
Les écoles d'ingénieurs Lieux de production et de reproduction d'une fraction des élites marocaines ?
}

\author{
Grazia SCARFO-G HELLA B
}

Mekki Merrouni, qui a réalisé une recherche sur les plans et les réformes mis en œuvre par le gouvernement marocain depuis l'indépendance du pays en 1956, raconte comment la décision de créer un système de grandes écoles s'est consolidée lors du Colloque des Chênes - colloque sur la politique éducative marocaine - qui a eu lieu au Maroc en avril 1964 :

«La mission de l'enseignement supérieur doit-elle être la diffusion de la haute culture dans le
cadre d'un systeme facultaire ouvert au libre choix des étudiants ou bien la formation des
cadres dont l'Etat a besoin, ce qui implique une orientation des étudiants en fonction des
besoins du pays et la priorité aux grandes écoles? Le ministère de l'Education nationale
penche pour la deuxième option : Ie M aroc a des besoins vitaux en cadres, il dispose de moyens
insuffisants et n'a pas de temps à gaspiller dans des formations hétéroclites et incertaines. »1

Ces écoles devaient s'inspirer ouvertement du modèle français : l'économiste marocain Khalid El Ouazzani souligne cette dimension récurrente du système marocain «que ce soit durant le protectorat ou depuis l'indépendance, l'héritage et l'influence de la politique éducative de la métropole se retrouvent à tous les degrés ${ }^{2}$. Dès 1959, la création de l'Ecole M ohammedia des ingénieurs (I'EMI) à Rabat anticipait, de fait, cette orientation politique. Depuis lors, d'autres écoles ont vu le jour : I'Institut national de statistique et d'économie appliquée (INSEA) en 1961, I'Institut agro-vétérinaire Hassan II (IAV) en 1966, I'Ecole nationale forestière des ingénieurs (ENFI) en 1968, I'Institut national des postes et télécommunications (INPT) en 1969, I'Ecole Hassania des travaux publics (EHTP) en 1971, etc.

Aujourd'hui, le Maroc compte une quinzaine d'écoles publiques qui délivrent le titre d'ingénieur d'Etat et dont la plupart ont été créées entre la fin des années 1950 et le début des années 1970. A vec la participation des établissements étrangers, notamment 
celle des grandes écoles françaises, près de 25000 ingénieurs marocains ont été formés, soit un taux de 8,6 ingénieurs sur 10000 habitants.

\section{LES HYPOTHĖSES DE TRAVAIL}

La Francea forméenviron 3000 ingénieurs marocains depuis les années 1960. Selon des données récentes, moins de $60 \%$ de cette population serait rentrée au Maroc ${ }^{3}$. Les écoles les plus représentées sont l'Ecole polytechnique, l'Ecole nationale des ponts et chaussées, I'Ecole centrale (Paris), I'Ecole supérieure d'électricité (SUPELEC), I'Ecole nationale supérieure des mines (Paris), l'Ecole nationale supérieure des télécommunications.

Les ingénieurs marocains représentent-ils l'une des fractions des élites sociales au Maroc ? La question posée conduit à chercher à comprendre les stratégies de reproduction des élites marocaines avec une attention particulière portée aux écoles d'ingénieurs en tant que lieu de passage de certaines fractions de ces élites. C'est une hypothèse qui reste à vérifier par une étude du devenir professionnel des ingénieurs.

Plusieurs travaux récents sur le système éducatif ${ }^{4}$ reconnaissent une dualité des lieux de production et de reproduction des élites marocaines formées dans les années 1960 : l'étranger, d'une part, et notamment un certain nombre de grandes écoles d'ingénieurs, comme par exemple l'Ecole polytechnique ou l'Ecole nationale des ponts et chaussées ; et, d'autre part, quelques institutions marocaines, I'EMI ainsi que les facultés de médecine et de droit.

Avec l'explosion récente des effectifs de l'enseignement supérieur, les facultés de droit se sont néanmoins transformées en «facultés de masse». La composition de leur public s'est modifiée à tel point qu'elles ne semblent plus assurer l'ancienne fonction de formation de «l'élite». Parallèlement, autour des années 80 , le secteur privé s'est présenté comme un nouvel acteur. En effet, certaines catégories de jeunes Marocains, appartenant aux milieux sociaux les plus favorisés, décident d'accomplir leur formation supérieure en suivant des études de gestion dans les écoles privées marocaines.

On peut ainsi s'interroger sur le devenir des écoles d'ingénieurs. Est-ce que les ingénieurs, ceux qui se sont formés dans les années 70, 80 et 90, gardent aujourd'hui - à l'instar de leurs homologues diplômés dans les années 60 - une place dans l'espace du pouvoir? 
N os hypothèses sont les suivantes :

1) Les écoles marocaines d'ingénieurs ne représenteraient plus un lieu de passage, ni un lieu de production des différentes fractions des élites au Maroc; et leur public, principalement issu des milieux populaires, n'atteindrait plus de très hauts niveaux dans leur carrière professionnelle. Ceci n'impliquerait pas pour autant que tous les ingénieurs soient de plus en plus éloignés du pôle du pouvoir.

2) Les grandes écoles d'ingénieurs françaises continueraient à représenter, en effet, un lieu de reproduction d'une partie des élites marocaines.

Pour vérifier ces hypothèses, nous nous proposons de passer par plusieurs étapes :

1) Déterminer, par questionnaire, le type de population transitant par certaines écoles marocaines d'ingénieurs ;

2) D'après l'étude d'un échantillon de jeunes Marocains inscrits durant les dernières années dans les grandes écoles françaises d'ingénieurs, mieux identifier les origines sociales des futurs ingénieurs formés à l'étranger ;

3) Constituer un échantillon d'ingénieurs travaillant au Maroc et qui appartiennent à des promotions scolaires relativement récentes. Les promotions scolaires de 1970, 1980 à 1990 seraient les plus pertinentes pour savoir si des ingénieurs - qui aujourd'hui ont entre 30 et 50 ans - occupent une place au sein des élites marocaines. Cet échantillon devra faire l'objet d'une étude par entretiens;

4) Distribuer des questionnaires auprès d'un nombre plus important d'ingénieurs (tous âges et promotions confondus) en place dans l'administration, dans les entreprises, etc., pour en retracer le cursus professionnel, scolaire et les origines socio-économicoculturelles. Dans ce cas, il faudra aussi prendre en considération la catégorie de «l'ingénieur maison », c'est-à-dire celui formé au sein de l'entreprise;

5) A travers une analyse documentaire, rendre compte des transformations survenues depuis l'indépendance dans le tissu économique et social marocain, au sein du système scolaire, ainsi que dans le système des bourses pour les études au Maroc et à l'étranger. L'analyse du système des bourses servira en particulier à saisir l'évolution des politiques scolaires relatives à l'accès aux différents systèmes d'enseignement supérieur local et étranger.

Pour l'heure, les étapes 3 et 5 ont été engagées. Aussi cette communication concernera-t-elle, en particulier, les premiers résultats des entretiens semi-directifs effectués auprès d'ingénieurs diplômés de grandes écoles françaises et marocaines appartenant aux promotions scolaires 1970-90. 


\section{NGÉNIEUR D'ÉCOLE VERSUS INGÉNIEUR MAISON}

A présent, une dizaine d'entretiens ont été réal isés auprès d'ingénieurs diplômés de I'Ecole polytechnique (X-Ponts), de l'Ecole nationale des ponts et chaussées, de l'Ecole des mines (Nancy), de I'Ecole Hassania des travaux publics (Casablanca), de l'Ecole M ohammedia des ingénieurs (Rabat). Une seule femme a fait l'objet d'un entretien 5 .

Lors de cette phase d'exploration du terrain et du sujet, d'autres hypothèses sont venues enrichir les premiers questionnements :

- L'identité socioprofessionnelle de l'ingénieur diplômé d'école serait forte et tributaire de la formation reçue ;

- Le diplôme serait l'un des critères fondamentaux pour définir le « vrai ingénieur », selon l'expression consacrée par certains d'entre eux ;

- Le secteur public resterait, aujourd'hui encore, l'une des voies privilégiées par les ingénieurs diplômés, ainsi qu'un lieu de reproduction et de passage de certaines fractions des élites marocaines ;

- En ce qui concerne le développement socio-économique du pays, les ingénieurs se percevraient comme des agents actifs de ce processus.

Afin de vérifier ces hypothèses, il nous est apparu nécessaire d'élargir l'échantillon des entretiens et d'y introduire les « ingénieurs maison ». N ous comptons ainsi rencontrer une trentaine d'ingénieurs marocains :

- Pour retracer leur trajectoire scolaire et professionnelle : les ingénieurs marocains sont-ils des « hommes de pouvoir » ? Est-ce leur diplôme et/ ou l'appartenance à un milieu social déterminé qui permet d'expliquer ce qu'ils sont devenus ? Ce point, particulièrement significatif dans le cadre d'une enquête sur la formation des élites, n'est pas analysé ici vu le faible nombre d'entretiens effectués.

- Pour comprendre comment les ingénieurs se perçoivent et saisir ainsi les modalités de leur(s) identité(s) socioprofessionnelle(s) ;

- Pour analyser, dans un premier temps, lecritère de définition du « vrai ingénieur » ou du « faux ingénieur » (selon la perception des intéressés) : le diplôme, le travail sur le tas, etc. ? Si ce critère est le diplôme, quelle est la formation idéale? Comment perçoiventils les écoles françaises et marocaines ? Existe-t-il entre les établissements de l'H exagone et ceux du Maroc des éléments de distinction fondamentaux ? Les divers critères seront croisés par la suite avec le type d'école ou de formation diplômante.

- Pour analyser l'existence ou non d'une carrière-type d'ingénieur (en fonction des perceptions des enquêtés), que cela soit dans les entreprises publiques et privées ou dans 
les administrations. Comment les ingénieurs ayant poursuivi une carrière administrative la perçoivent-ils ? La considèrent-ils commeéloignée de leur fonction fondamentale - c'està-dire de leur fonction technique - ou, en revanche, comme une trajectoire permettant à l'ingénieur de voir réaliser ses options en matière de développement du pays ?

- Pour comprendre le rôle del'ingénieur dans le développement socio-économique. En quoi consiste-t-il de façon pratique ? Est-il l'un des agents qui pourraient déclencher un processus de développement endogène ou renforce-t-il la dépendance vis-à-vis de l'étranger?

Les résultats préliminaires de cette recherche ne concernent que des entretiens réalisés avec des ingénieurs diplômés de grandes écoles. II reste à comparer les différentes réponses obtenues avec celles des « ingénieurs maison ».

Par ailleurs, les hypothèses que nous formulons sont encore provisoires. Toutefois, une des premières idées fortes qui se dégage des entretiens, tous milieux sociaux et écoles d'ingénieurs confondus, est relative aux caractéristiques essentielles distinguant l'ingénieur des autres professions : l'ingénieur serait une personne qui travaillerait beaucoup, méthodiquement, et qui résoudrait les problèmes dans la mesure où il les aborde par une approche scientifique, cartésienne. Cette approche scientifique dans la solution des problèmes le distinguerait nettement de «l'ingénieur maison », mais aussi du simple licencié. A la base de cette perception, plutôt que l'école d'ingénieurs, on retrouverait le cursus de formation suivi et les classes préparatoires dans lesquelles l'intéressé a été « intégré ».

«Chez nous, il y a beaucoup d'ingénieurs qui se sont formés sur le tas : je sens que leur raisonnement n'est pas juste, qu'ils font des analyses en sautant des étapes. Ce qui leur fait défaut est le raisonnement (... ). Le diplôme est fondamental en termede compétence, de savoir, de théorie. Sur le tas on comprend les vrais problèmes. $M$ ais la formation de base est nécessaire pour comprendre tous les phénomènes. On peut former des ingénieurs pour des tâches spécifiques, mais la formation sur le tas ne remplace pas la formation de base (... ). N 'importe qui à un niveau moyen pourrait faire ce que je fais, mais la formation de base garantit une façon de raisonner, une approche. $\gg 6$

Le passage dans les classes préparatoires et certainement le concours national semblent avoir renforcé une autre idée : celle de l'appartenance à une élite scolaire :

«Parmi mes camarades au lycée, j'étais l'un des meilleurs. Pour cela, je me suis orienté tout naturellement vers les classes préparatoires (...). En "prépa", on a su qu'on était une petite élite... II suffisait qu'on soit les meilleurs pour avoir les classes préparatoires. »

On retrouve ces affirmations à l'identique dans les discours de ceux qui sont partis en France aussi bien que de ceux qui ont passé leur concours au Maroc et ont intégré une école marocaine ou une école française. 
L'obtention du diplôme d'ingénieur n'ébranle pas, bien au contraire, cette certitude qui se concrétise dans des attentes très importantes au niveau de la carrière. A la sortie de l'école, chaque interviewé était convaincu qu'il se préparait à une carrière brillante et rapide. II faut rappeler que les exemples de jeunes ingénieurs occupant des postes très importants, aussi bien dans le secteur public que dans le privé, sont nombreux au Maroc.

\section{SYSTÈmes ÉduCATIFS NATIONAUX ET STRATÉGIE de CARRIÈre}

Lors de la reconstruction de la carrière professionnelle, les différences entre diplômés d'écoles marocaines et diplômés d'écoles françaises commencent cependant à se percevoir. Les polytechniciens ou les ingénieurs des Ponts ou des Mines changent, recherchent et changent encore d'emploi. Ils ne se résignent pas devant des évolutions de carrière qui ne sont pas - à leurs yeux - assez rapides. Finalement, on en retrouve une bonne partie, dix ou quinzeans après leur sortie del'école, dans l'administration publique, dans des postes de directeurs centraux au sein des ministères ou de directeurs généraux de grandes offices publics, occupant à la fois des fonctions techniques et managériales.

Ceux qui ont obtenu les postes de directeurs centraux ou généraux le plus rapidement (trois/ quatre ans après leur retour au Maroc) appartiennent à de grandes familles marocaines. Souvent, leurs parents, leurs frères et sœurs ont suivi eux aussi une formation supérieure à l'étranger ; et, quand ils travaillent, ils occupent, dans le privé comme dans le public, des postes importants.

Nous nous proposons d'approfondir l'analyse du rôle respectif de la famille et du diplôme dans la construction d'une élite formée dans les grandes écoles françaises. Cette filière ne fait-elle que confirmer une donnée établie ou sert-elle aussi de vecteur de mobilité sociale? Est-elle un lieu de production ou plutôt de reproduction d'une fraction del'élite?

Les diplômés de l'Ecole Hassania de Casablanca, eux aussi, nourrissent également des rêves de promotion. Mais, face aux premiers obstacles d'emploi, leur attitude paraît différente: ils se plient à la dure réalitéet se résignent. Quinze ans après avoir obtenu son diplôme, l'un d'entre eux espère pouvoir atteindre le niveau de chef de service dans I'administration publique. Pour lui, l'échec est double. II voulait être un « vrai ingénieur », faire du chantier, ou travailler dans un bureau d'études ; et «surtout pas dans le public » et « surtout pas dans l'administration ». 
Pour les diplômés des grandes écoles françaises, faire une carrière dans le secteur public semble représenter le but à atteindre : «Moi, je me sens mieux dans le public, parce qu'il me semble contribuer le plus au développement du pays (...). Le public est plus honorifique pour la personne».

Deux questions dépendantes l'une de l'autre restent à approfondir : 1) le secteur public représente-t-il encore aujourd'hui un objectif dans la carrière des ingénieurs marocains, vu la perte de certains avantages en nature tels que le véhicule ou le logement de fonction qui compensaient souvent un salaire moins intéressant que dans le privé ? 2) le secteur public représente-t-il encore aujourd'hui la « voie royale » del'élite?

L'image de l'ingénieur opérationnel qui, tout en ayant des tâches managériales, ne doit pas s'éloigner des tâches techniques, est présente aussi bien dans les idées exprimées par les diplômés des grandes écoles françaises, que dans celles des diplômés au Maroc.

«Beaucoup d'ingénieurs des grandes écoles ne font pas de l'ingénierie. Ils ne font que de l'administratif, ils font un travail qui n'a plus rien à voir avec leur formation initiale, dans les banques, les assurances. Nous perdons nos compétences initiales. C'est une grande frustration. 》

Un autre essaie d'expliquer ce phénomène :

«Le parcours classique devrait être le suivant : l'ingénieur devrait s'insérer dans une unité de production, passer par toutes les étapes, la maintenance, etc. et très vite avoir la responsabilité de l'unité de production. A près, il doit devenir directeur technique. Mais le travail technique n'est pas val orisé au $M$ aroc. Un directeur technique est moins bien vu qu'un directeur financier, qu'un directeur commercial/marketing. »

Un point semble rapprocher les uns et les autres: l'existence d'un réseau de « camarades » d'écoles. Qu'il s'agisse d'une véritable amicale - comme celle des polytechniciens ou celle des ingénieurs de l'Ecole nationale des ponts et chaussées du $M$ aroc - ou de relations informelles, ce réseau existe et il joue un rôle très important dans la construction de la carrière de chaque interviewé :

«J'étais encore en France quand jel'ai rencontré (il s'agit d'un ancien camarade de l'école) et
c'est lui qui m'a donné envie de rentrer, car on pouvait faire des choses intéressantes au M aroc
(...). A près, il m'a demandé si j'étais satisfait de mon poste au M aroc, et, vu mon
insatisfaction, il m'a fait sa proposition, quej'ai acceptée et l'ai suivi (...). C'est un camarade
de l'école qui m'a proposé mon premier poste (...) C'est encore à travers un camarade quej'ai
changé de poste (...). Tous mes camarades m'ont conseillé d'aller voir au niveau du public. »

Comment les diplômés en France perçoivent-ils les diplômés des écoles marocaines,

et inversement ? Le manque de confiance en soi de la part des lauréats des écoles marocaines est sans doute la caractéristique la plus citée par les diplômés en France. Suivent les préjugés de l'environnement qui font que l'on embauche plus facilement des diplômés de l'école française, on leur confierait plus de responsabilités et on aurait plus 
confiance dans leurs compétences. En dernier, ils citent, un peu mal à l'aise, la qualité de la formation marocaine «peut-être moins bonne ». En revanche, les diplômés au Maroc affirment qu'il y a certainement une différence de mentalités : « Les diplômés en France ont une autre ouverture d'esprit, mais pour ce qui est des compétences, cela dépend de la personne ». Eux aussi admettent que, s'ils étaient employeurs, ils embaucheraient avec une plus grande confiance un ingénieur formé... à l'étranger.

\section{DéVELOPPEMENT ENDOG ÈnE VERSUS DÉVELO PPEMENT DÉPENDANT}

En ce qui concerne le point relatif au développement socio-économique du pays, ces premiers entretiens nous permettent de dégager quelques données intéressantes. Tout d'abord, les lauréats des écoles françaises affirment que le rôle de l'ingénieur est, non seulement très important, mais irremplaçable :

« On souffre d'un taux d'encadrement très faible au M aroc. S'il y avait partout des ingénieurs, on ferait beaucoup d'économies (... ). D ans le cadre du développement, le savoirfaire, les compétences, l'ingénierie ont largement leur place. En particulier, l'ingénieur doit intervenir au niveau dela production et dela recherche. »

Un autre aspect, très intéressant concerne la problématique relative au développement endogèneversus développement exogène ${ }^{7}$. En effet, certains interviewés soulignent le risque d'un développement extraverti, qui se concrétiserait par exemple à travers l'établissement de relations privilégiées avec des interlocuteurs français :

«Quand je travaille en collaboration avec une entreprise française dans laquelle souvent j'ai des chances de trouver comme interlocuteurs des ingénieurs camarades de l'école, il est clair que les rapports sont plus simples : nous parlons le même langage ! »

A l'instar de cet ingénieur de l'Ecole nationale des ponts et chaussées, l'un de ses condisciples réitère l'idée que l'imprégnation de la culture du pays de formation est déterminante :

«Si certains pays nous financent les études, ce n'est pas pour nos beaux yeux : un ingénieur formé aux Etats-U nis, ne pense qu'américain ; un ingénieur formé en France ne pense que français. Nous risquons d'avoir une tendance à l'imitation. Logiquement, ceux qui sont formés au $M$ aroc devraient penser marocain. Encore faut-il qu'ils aient confiance en euxmêmes. 》

Dans ces récits, on ressent l'appréciation de la formation made in France, même si celui qui parle porte un regard critique vis-à-vis de solutions importées et révèle la volonté de se détacher d'une emprise culturelle trop importante. On y perçoit l'assurance des « dominants » qui peuvent critiquer un certain monde parce qu'ils y appartiennent. Et cette assurance semble leur donner la force d'envisager la construction d'un modèle de développement différent, approprié au Maroc. 
Les diplômés du pays affirment également l'importance de l'ingénieur pour le développement national. Tout en se disant «frustrés » parce qu'ils n'ont pas pu y contribuer à la hauteur de leurs compétences.

En guise de conclusion provisoire, nous rappellerons que ce premier compte rendu d'un travail d'enquête sur les ingénieurs marocains n'a pour ambition que de tester la validité de nos questionnements. Certains points permettent, d'ores et déjà, d'ouvrir des pistes de recherche. Plutôt que tirer des conclusions - tâche impensable étant donné le nombre très faible d'entretiens - on mettra en valeur les points suivants :

1. Tous les interviewés - milieux sociaux et écoles confondus - proposent dans leur récit une image précise de l'ingénieur : celle d'une personne qui analyse et résout les problèmes grâce à une méthode scientifique, grâce à l'application d'un raisonnement cartésien. Cette approche rigoureuse, qui en fait des agents irremplaçables du processus de développement socio-économique du pays, leur vient de leur parcours scolaire : baccalauréat scientifique, classes préparatoires, école d'ingénieurs. Personnellement, j'ai pu vérifier auprès des étudiants de troisième et dernière année de l'Ecole Hassania des travaux publics, tout au long de mes cinq années d'enseignement, que cette même image est fortement ancrée dans l'esprit de ces jeunes Marocains.

2. Les ingénieurs, ainsi que mes élèves, se perçoivent comme une élite scolaire et scientifique. Celle-ci semble dépasser les différences relatives aux origines sociales ainsi qu'aux écoles d'appartenance. Une élite qui a certainement ses dominants et ses dominés, mais qui - si elle existe réellement - pourrait générer des perturbations au niveau des mécanismes de reproduction plus classiques. Ce point devra faire donc l'objet d'une étude particulièrement attentive.

3. Un troisième constat se rapporte à l'identité de l'ingénieur : celle des interviewés semble être forte, mais les entretiens auprès d'«ingénieurs maison » devrait permettre de comprendre si l'on est confronté à des identités socioprofessionnelles différentes liées à des formations différentes (diplôme, formation sur le tas, etc.) ${ }^{8}$. Tous les interviewés semblent regretter de ne pas s'adonner à un travail technique. L'ingénieur des grandes écoles utilise peu ses compétences de base. Le lien entre la formation reçue et la carrière poursuivie semble se faire alors non pas au niveau de l'acquisition et l'utilisation de compétences spécifiques, mais plutôt au niveau de la simple existence d'un titre : c'est donc la possession du diplôme d'ingénieur - certification symbolique de compétences qui influencerait l'évolution des carrières. 
4. La corrélation entre l'évolution de la carrière des ingénieurs marocains et le type de formation représente un autre point fondamental à creuser. Le secteur public se présente encore aujourd'hui comme l'un de lieux d'accueils des ingénieurs marocains, qu'ils viennent des grandes écoles françaises ou marocaines. Représente-t-il un but recherché ou plutôt un second choix résigné ? Les premiers entretiens nous conduisent plutôt à choisir la première option, même si cette conclusion peut paraître prématurée. L'enquête devra aussi essayer de comprendre si l'intégration des ingénieurs dans le secteur public demeure « la voie royale » du recrutement del'élite ou si, désormais, elle est le symptôme d'un déclassement des ingénieurs, à l'instar de ce qui s'est passé dans d'autres pays arabes 9 .

5. A propos de la question del'émergence d'un nouveau profil d'ingénieur « innovateur » ou « développeur », certains interviewés mettent en garde contre le danger que représente la « dépendance » technologique, sociale, culturelle et économique à l'égard de l'étranger en général, et de la France en particulier. Aussi aspirent-ils à devenir des agents véritables d'un développement endogène. Par ailleurs, la reconstruction détaillée de leur carrière - qui constitue l'un des thèmes abordés lors de l'entretien - devrait nous permettre de comprendre dans quelle mesure les ingénieurs marocains «mettent en œuvre les capacités d'innovation et d'initiative, nécessaires pour prendre la place des experts étrangers $\gg 10$.

6. Nous devons enfin nous interroger sur l'existence éventuelle de différences significatives entre l'influence exercée sur le processus de dével oppement du pays par un ingénieur formé en France et celle exercée par un ingénieur diplômé au Maroc. La tendance à avoir un comportement mimétique de la part des lauréats des grandes écoles françaises représente-t-elle une réalité ? Pourrait-elle exister aussi parmi les ingénieurs formés au Maroc, en raison par exemple d'une dépendance structurelle du système scolaire marocain vis-à-vis du système scolaire français?

\section{N OTES}

1 MERROUNI M., Le problème de la réforme dans le système éducatif marocain, Ed. Okad, Rabat, 1993, p. 59. Cette recherche a été réalisée dans le cadre d'un programme de coopération entre la faculté des lettres et des sciences humaines de Rabat et la Fondation Konrad Adenauer. 1991, p. 17.

2 EL OUAZZANI K., Coûts et rendements de l'enseignement supérieur au M aroc, Ed. ESG, Casablanca,

${ }^{3}$ AUDRIC T., «Les Etudiants marocains dans les écoles d'ingénieurs françaises », Paris, ministère des Affaires étrangères, Sous-Direction de la Coopération universitaire et scientifique, mai 2000, p. 6. 
4 EL OUAZZANI K., op.cit. ; BAINA A., Le système de l'enseignement au Maroc, trois tomes, Ed. Maghrébine, 1982 ; SALMI J., Crise de l'enseignement et reproduction sociale au M aroc, Ed. Maghrébine, 1985.

5 Commencer par cette étape s'explique pragmatiquement : grâce aux contacts personnels et professionnels que nous avons noués avec certains ingénieurs marocains, il a été possible de faire ressortir le bien-fondé d'une étude sur les ingénieurs maghrébins et obtenir de ce fait des rendez-vous. Les entretiens ont été aussi l'occasion de parler aux interviewés du questionnaire. De cette façon, la prochaine étape devrait pouvoir être franchie sans trop de difficultés.

6 Les entretiens réalisés ont été effectués entre le mois de novembre et le mois de décembre 2000.

7 «La production de biens et de technologies viseà l'origine la résolution de problèmes d'adaptation et de survie des hommes organisés dans un cadre social. Cela implique que toute société est productrice de biens et de techniques nécessaires à la réalisation de ses objectifs (...). Mais des ruptures peuvent survenir dans le processus historique de production sociale notamment lorsqu'une société se trouve dominée par une autre. La culture dominante tente général ement d'effacer les cultures dominées. La société dominée perd de ses capacités créatives et se tourne vers la société dominante pour trouver les moyens de résoudre ses problèmes de survie, d'adaptation ou de développement. Cet élan porté vers l'imitation est à l'origine de plusieurs dysfonctionnement et d'inadaptation des systèmes techniques de production, aux besoins sociaux du grand nombre qui restent insatisfaits ou mal satisfaits. » in ZGHAL R., « La globalisation, et les impératifs d'appropriation de la technologie », Cahiers de l'ER GE, n 6, décembre 1999, p. 11.

Dans la ligne de ces réflexions se dégage le concept de développement endogène en tant que capacité du pays à résoudre ses propres problèmes d'adaptation «à sa façon » et qui s'opposeà l'imitation, voireà la dépendance (culturelle, sociale, économique...), vis-à-vis de la société dominante.

8 « Un grand nombre de techniciens postulent aux fonctions d'ingénieur, mais aussi au titre. Les modes de sélection des candidats à des formations continues, I'une de type NFI et l'autre de type universitaire, s'opposent. La première paraît plus rigoureuse et scientifique, la seconde plus artisanale. A travers cette opposition, on voit se dessiner deux figures professionnelles nouvelles, celle du "quasiingénieur " et celle de l'" équivalent-ingénieur ". Elles vont venir s'ajouter et se combiner à la partition classique entreingénieur d'école et ingénieur maison. » in GADEA C., LOUBET P., ROQUET P., «Ingénieurs en puissance », in Formation Emploi, $n^{\circ}$ 55, juillet-septembre 1996, p. 43. Voir aussi, KALCK P., GAUTIER F., « Devenir ingénieur ou cadre supérieur - Le rôle des formations promotionnelles » et BOUFFARTIGUE P. MARRY C., «Formation d'ingénieurs, Le modèle de l'élite en question » in Formation Emploi, $n^{\circ} 53$, janviermars 1996, pp. 15-19 et pp. 3-13.

9 Intervention d'Elisabeth Longuenesse à la réunion de lancement du programme de I'IRMC «Ingénieurs et société au M aghreb », 15-16 octobre 1999.

10 LONGUENESSE E., « Les ingénieurs au Maghreb et au Moyen-Orient. Politiques de développement et nouveaux acteurs sociaux », in LONGUENESSE E. (éd.), Bâtisseurs et bureaucrates. Ingénieurs et société au M aghreb et au M oyen-O rient, Maison de l'Orient, Lyon, 1990, p. 19. 

La relation formation-emploi 



\title{
Les évolutions de la formation et du statut social des ingénieurs dans l'Algérie indépendante
}

\author{
M ustapha H A D D A B
}

II est bien connu que parallèlement à la conduite d'une politique économique principalement orientée vers la constitution d'une industrie étatisée destinée à occuper une position économique déterminante, les dirigeants algériens, durant approximativement la période qui va de la fin des années 1960 à la fin des années 1970, se sont efforcés de développer ou de créer différentes institutions de formation de cadres et particulièrement d'ingénieurs, dans le but de mettre à la disposition des unités de production déjà constituées, ou en cours d'édification, les personnels et en particulier les cadres de haut niveau nécessaires.

Quelques chiffres peuvent permettre de représenter l'importance des réalisations visant à former en des temps relativement courts, des effectifs considérables d'ingénieurs. En 1981, le nombre de diplômés sortant annuellement des établissements de formation d'ingénieurs, n'était encore que de 1 120. Ce nombre s'élève à 6000 en 1991, sur 25600 diplômés pour l'ensemble de I'Université, (soit 23,4%). Entre 1981 et 1991, 43000 ingénieurs avaient été formés aussi par les différents établissements de l'enseignement supérieur. Les diplômés dans les filières technologiques sont au nombre de 12519 en 1998, et ils représentent 31,6 \% de l'ensemble des diplômés de la même année. Entre 1992 et 1998, 79444 ingénieurs ont été formés. Le nombre des étudiants des filières technologiques, du niveau de la graduation ${ }^{1}$, s'élevait en 1998-1999, à 74917 soit 17,68 \% du total des inscrits à ce niveau, dans l'enseignement supérieur². 


\section{CONSTITUTION D'UN ENSEMBLE SOCIAL hÉTÉROGÈnE}

II semble ainsi possible de dire qu'un groupe social relativement nouveau a été, d'une manière dans une large mesure volontariste, constitué dans la société al gérienne, en un temps relativement court. Parmi les élites al gériennes antérieures à l'indépendance, la figure de l'ingénieur n'apparaissait que faiblement. Les ingénieurs étaient d'ailleurs peu nombreux même dans la communauté européenne. Ainsi pourrait-on dire que dans leur perception et leurs pratiques sociales, les Algériens n'étaient guère nombreux à avoir acquis une connaissance concrète du personnage de l'ingénieur. II en est résulté une tendance à surévaluer l'importance de l'ingénieur dans l'imaginaire social.

Le corps des ingénieurs s'est constitué en Algérie principalement à partir de deux sources. La première filière regroupait l'ensemble des institutions dans lesquelles s'est formé un nombre réduit d'ingénieurs, durant la période de la lutte de libération nationale, et au début de l'indépendance. Une partie de ces ingénieurs, peu nombreux, avaient pu, à la suite d'itinéraires sociaux un peu particuliers, accéder, en partant de lycées al gériens de la période coloniale, à des formations d'ingénieurs souvent dans des grandes écoles françaises ; d'autres avaient pu, durant la guerre, bénéficier de bourses, pour se rendre, sous l'égide du Front de libération nationale (FLN) ou du Gouvernement provisoire de la république al gérienne (GPRA), dans diverses contrées, fréquemment des pays del'Est, pour y obtenir des diplômes d'ingénieurs. A l'indépendance, ces ingénieurs ont constitué un premier noyau de «technocrates », très proches du pouvoir tout en s'en distinguant, et qui se sont vu confier de lourdes tâches, et beaucoup de pouvoir, dans le processus de l'édification d'une importante industrie d'Etat.

La seconde filière de formation d'ingénieurs est constituée de l'ensemble des établissements d'enseignement supérieur, les uns dépendant du ministère de l'Enseignement supérieur, et d'autres placés sous la tutelle de divers ministères, qui ont été progressivement mis en place après I'Indépendance. Certains de ces établissements avaient été fondés durant la période coloniale, mais ils ont fait I'objet, après 1962, d'importantes modifications tant du point de vue de leur taille que de leur statut.

\section{UN ÂGE D'OR DES INGÉNIEURS?}

On peut sans doute dire que la période qui va de la fin des années 1960 à la fin des années 1970 fut, en Algérie, l'âge d'or des ingénieurs, et particulièrement de ces diplômés issus de diverses institutions appartenant à différents pays. II existe une sorte de 
correspondance entre la hiérarchie qui s'est établie au sein du corps des ingénieurs algériens et la chronologie des périodes de formation de ces ingénieurs. Les ingénieurs les plus anciens ont occupé et pour certains d'entre eux occupent encore, dans les institutions et les entreprises auxquelles ils appartiennent, les positions les plus élevées ; ils sont également les plus proches des plus hautes instances dirigeantes. Un certain nombre d'entre eux ont occupé des postes de ministre.

Ce sont, pourrait-on dire, les ingénieurs dont la nomination à des postes de direction est intervenue durant environ les dix premières années de l'indépendance qui ont bénéficié de la plus haute valorisation sociale de leurs diplômes. Les ingénieurs qui avaient ainsi pu accéder à la direction d'entreprises ou d'unités de production importantes ont bénéficié d'avantages matériels et symboliques considérables. Mais ils ont eu aussi le plus souvent à exercer leurs fonctions dans des conditions très difficiles, liées à de multiples facteurs tels que des formes d'inadaptation de l'environnement économique et social aux conditions de fonctionnement normales de l'entreprise, la qualification ou l'expérience insuffisantes des gestionnaires ou des techniciens moyens ou subalternes, les aléas dans les approvisionnements en matière première et aussi la nature souvent complexe et pourrait-on dire «surdéterminée » des conflits qui se produisent parmi les différentes catégories d'agents de l'entreprise.

Il était courant d'entendre dire, même durant la période où ils étaient fortement recherchés par les institutions économiques, que les ingénieurs étaient mal «utilisés » et qu'au lieu d'être affectés à des postes proches des activités de production dans les entreprises et les usines, ils se voyaient attribuer des fonctions de gestion administrative. On peut se demander si cette tendance dont tout porte à penser qu'elle était réelle, était due non pas tant à des dysfonctionnements dans la gestion des cadres disponibles, mais plutôt aux effets des caractéristiques objectives des conditions de travail dans les entreprises : celles-ci étaient en effet rarement engagées dans un environnement de compétition économique qui aurait contraint à chercher à maximiser l'efficacité des processus de production, I'organisation du travail et la productivité, au sein des usines et des ateliers.

Autour des gestionnaires à qui I'on avait confié la direction de grandes entreprises du secteur d'Etat, se constituaient des équipes de cadres dirigeants, parmi lesquelles se trouvaient fréquemment des ingénieurs. Les groupes de gestionnaires des unités de production et des sociétés composant le secteur industriel public se sont constitués progressivement selon des processus relevant de la cooptation et de regroupements par 
affinité ; les équipes dirigeant telle ou telle entreprise industrielle ou telle usine comportaient souvent une proportion considérable d'ingénieurs ayant fréquentéla même école, la même université, ou le même institut ; certaines entreprises ou sociétés nationales étaient connues pour être dirigées par des cadres issus plutôt des universités américaines, d'autres plutôt par des cadres formés en France ; il y avait dans la direction de plusieurs autres des ingénieurs venant de divers établissements de formation des pays del'Est.

\section{ORIGINE SOCIALE DES INGÉNIEURS}

On peut formuler l'hypothèse que la majorité des ingénieurs algériens appartiennent aux catégories sociales qui ont le plus bénéficié des promotions sociales qui se sont produites après I'Indépendance, promotions qui ont touché, grâce au fort dével oppement du salariat, et à la politique de démocratisation du système éducatif, des fractions modestes des classes moyennes. La formation d'un groupe relativement nombreux d'ingénieurs paraît plus directement liée au projet d'une Algérie industrialisée et modernisée que ne l'était l'extension du corps des médecins ou des architectes; la politique économique et sociale suivie à partir de la fin des années 1960 tendait à substituer une Algérie dans laquelle l'industrie serait dominante à l'A Igérie antérieure à I'indépendance, essentiellement agricole. Dans l'idéologie économique et politique qui a prévalu à la fin des années 1960, il y avait un côté saint-simonien, qui a fortement contribuéà donner au paradigme de l'ingénieur une forte valorisation sociale.

Si l'augmentation considérable des effectifs d'étudiants dans les filières universitaires de technologie et dans les Ecoles ou Instituts destinés à la formation d'ingénieurs, a permis à des jeunes appartenant à des fractions des classes moyennes de bénéficier de véritables promotions, il semble toutefois que l'obtention de diplômes d'ingénieurs ait été plus fréquente parmi les catégories socioprofessionnelles bénéficiant déjà d'un capital socio-économique et culturel important.

Ainsi le Recensement général de la population et de l'habitat de 1989, permettait d'observer que sur les 49718 ingénieurs que comprenait alors la population algérienne, 17 666, soit 35,5\%, étaient issus de la catégorie sociale des « cadres supérieurs » et des «professions libérales ». Beaucoup moins nombreux étaient les ingénieurs issus des autres catégories qui regroupent pourtant des populations plus nombreuses : 10,3 \% des ingénieurs appartenaient aux familles de «cadres moyens », 8,9 \% à la catégorie des « indépendants », 5,9 \% à celle des « ouvriers », 3,6 \% à celle des « employés », et 1,5\% 
étaient issus de la catégorie sociale des « employeurs ». En outre sur les 49718 ingénieurs recensés alors, 4033 seulement étaient des femmes, soit $8 \%$ 33,6 \% de ces ingénieurs de sexe féminin sont issues de la catégorie des « cadres supérieurs » et de celle des «cadres moyens ». II y a ainsi des écarts entre l'origine sociale des ingénieurs garçons et des ingénieurs filles, qui autorisent à formuler l'hypothèse que ces dernières sont plus fréquemment que les premiers d'une origine sociale plus modeste.

Les résultats déjà publiés du dernier Recensement général de la population (1998) ne permettent pas d'établir des relations (même sommaires) entre les différentes catégories de diplômés et leur appartenance socioprofessionnelle ${ }^{3}$. Bien des indices conduisent à penser toutefois qu'il s'est produit une certaine stabilisation dans la « démocratisation » relative du recrutement des étudiants des filières ou des établissements préparant à des diplômes d'ingénieur. On peut sans doute faire I'hypothèse qu'à partir d'un certain moment à déterminer, les promotions de nouveaux étudiants et de nouveaux diplômés dans ces filières sont, dans une large mesure, issues de couches sociales ayant bénéficié dans les années 1960 et 1970 d'une mobilité sociale ascendante considérable et qui, en quelque sorte, entrent dans une phase de reproduction. En outre, la saturation que connaissent beaucoup de spécialités technologiques, en particulier celles qui sont enseignées dans des Ecoles ou des Instituts réputés comme I'Ecole polytechnique, I'Institut national d'agronomie, I'Institut national d'informatique, etc. détermine des formes de compétition et de sélection entre les candidats à l'admission dans ces filières qui tendent à favoriser les jeunes issus des groupes sociaux les plus dotés en capital socio-économique et culturel.

\section{FORMATION D'INGÉNIEURS ET « DÉSINDUSTRIALISATION 》 DE L'A LGÉRIE}

Depuis quelques années semble se renforcer une situation d'inadéquation entre l'organisation de l'enseignement supérieur et les changements structurels qui se sont produits et se produisent encore dans l'économie al gérienne. Cette situation relève de ce que l'on pourrait appeler un processus d'hystérésis. L'Université continue à fonctionner selon la trajectoirequi lui avait ététracée dans les années 1970 ; la proportion d'ingénieurs issus des filières technologiques des universités, et des écoles d'ingénieurs, reste ainsi importante, parmi l'ensemble des diplômés de l'enseignement supérieur.

Il semble tout à fait légitime de se poser la question de savoir si le dynamisme relatif dont fait preuve aujourd'hui le secteur privé des petites et moyennes entreprises ne déterminera pas, à terme, une valorisation plus importante des formations de personnels qualifiés de niveau moyen que celle dont bénéficient ou bénéficieront les formations 
d'ingénieurs. Les données que l'on parvient à obtenir, et qu'il ne faut utiliser qu'avec beaucoup de prudence, indiquent que le nombre d'emplois dans le secteur privé a tendance à augmenter d'une manière significative.

On compterait ainsi, au 31 décembre 1999, 159507 entrepreneurs privés, employant 634375 salariés. De 1998 à 1999, le nombre de petites et moyennes entreprises aurait augmenté de 21000 unités environ. II est bien connu que le nombre de salariés déclarés est très inférieur au nombre de personnes effectivement employées dans les entreprises privées. (Le total des salariés du secteur privé s'élèverait, selon plusieurs sources, à environ 1200000 personnes.) A la différence des différentes branches du secteur privé qui font ainsi preuve d'un dynamisme considérable, le secteur public connaît différentes formes de stagnation voire de régression. Ainsi, selon des chiffres émanant du ministère del'Industrie et de la Restructuration, la production industrielle a accusé durant les neuf premiers mois de l'année 2000 une diminution globale de 1,3\%, avec des différences importantes selon les branches. De même, entre fin décembre 1999 et fin septembre 2000, le secteur industriel public a perdu environ 4000 emplois. Ces données, aussi sommaires soient-elles, conduisent à penser que l'évolution actuelle de la structure de l'économie algérienne implique une diminution du besoin en ingénieurs, plutôt que son augmentation.

Considérée du point de vue d'une politique éducative, cette situation soulève de difficiles problèmes : il est ardu en effet de trancher la question de savoir s'il faut s'efforcer de réduire les effectifs de nouveaux étudiants admis dans les filières de technologie des universités, des centres universitaires, ou dans les écoles d'ingénieurs, ce qui aurait pour effet d'augmenter le nombre déjà important d'étudiants dans les disciplines médicales et dans les disciplines littéraires ; ou s'il ne vaut pas mieux choisir de maintenir des taux d'effectifs importants dans les filières de formation d'ingénieurs, mal gré les difficultés d'accès à l'emploi que rencontrent ces derniers. II en résulterait que ces ingénieurs seraient portés à faire preuve d'initiative pour tirer parti de leurs compétences, en créant des entreprises, en s'associant à des projets de partenariat, etc. Le maintien d'un taux élevé de scientifiques, et surtout d'ingénieurs, parmi les diplômés de l'enseignement supérieur permettrait de ne pas renoncer au projet de modernisation et d'autonomisation de la société. La tentation de réduire les proportions d'étudiants orientés vers des formations d'ingénieurs est d'autant plus forte que ces dernières reviennent plus cher à l'Etat que les formations de lettres, de droit ou de sciences sociales. 


\section{LES DÉBOIRES DES INGÉNIEURS DEPUIS LE MILIEU DES ANNÉES 1980}

Les sociologues Elisabeth Longuenesse et Roland Vaast observent très justement que « la remise en cause du modèle "socialiste" jette les professions scientifiques dans une crise multiforme. Elle affecte profondément leur situation matérielle. Elleminore leur statut social : l'affairiste influent et le simple commerçant prennent le pas sur elles »4.

Pour mesurer et décrire les différentes formes de dévalorisation, évoquées ici par ces auteurs à propos de trois pays du monde arabe, qui affectent le groupe des ingénieurs en Algérie en même temps que d'autres groupes de professions scientifiques, il faudrait réunir beaucoup de données, qui ne sont guère aujourd'hui disponibles.

Quelques hypothèses peuvent néanmoins être formulées. Les processus d'affaiblissement de la condition et de la position sociale des ingénieurs atteignent moins fortement les groupes les plus haut placés dans cette catégorie professionnelle que ceux qui se situent plus bas dans la hiérarchie. Les ingénieurs qui font ou qui ont fait partie des milieux dirigeants des institutions administratives et économiques du pays préservent leur position sociale assez aisément grâce en particulier au capital de relations sociales qu'ils ont en général accumulé. Un nombre non négligeable de ces ingénieurs prennent leur retraite et constituent des bureaux d'étude ou des cabinets de conseil.

Moins favorable est la situation des ingénieurs plus jeunes qui occupent ou occupaient des postes plus modestes dans les sociétés nationales et les différentes unités de production du secteur public. Les problèmes de trésorerie, les obstacles à la commercialisation des produits réalisés, les conflits naissant des nécessaires compressions de salariés, les suspicions que suscitent les méthodes de gestion pratiquées, etc. affectent souvent durement cette catégorie d'ingénieurs, aussi bien matériellement que moralement. Quant aux diplômés les plus récemment sortis des filières de formation technologique, ils se trouvent confrontés à une offre d'emploi quantitativement et qualitativement insuffisante. Ils sont conduits à accepter diverses formes de déclassement. C'est ainsi qu'un nombre considérable d'entre eux a obtenu des postes d'enseignants dans différents établissements (lycées techniques et technicums, voire institutions d'enseignement professionnel) ; le nombre d'enseignants titulaires d'un diplôme d'ingénieur d'Etat dans le cycle secondaires'élevait en 1997 à 2872 sur les 52210 enseignants que comptait al ors le cycle (soit 5,5\%).

Beaucoup de diplômés des filières technologiques de l'enseignement supérieur sont contraints de se livrer à des activités ou d'occuper des fonctions correspondant à des niveaux de qualification inférieurs à ceux qu'ils ont atteints par leurs études ; certains ingénieurs exploitent des «pizzerias », d'autres tiennent des magasins d'articles 
électroniques ou d'installation d'antennes pour la télévision, etc. ; lorsque les familles auxquelles ils appartiennent possèdent des entreprises commerciales, artisanales ou industrielles conséquentes, des ingénieurs, dans une proportion qui ne semble pas être négligeable, s'intègrent aux activités de ces entreprises, activités ayant souvent peu de rapports directs avec la nature de la formation reçue. On peut en outre voir un indice de l'affaiblissement de la situation sociale des formations d'ingénieurs dans le fait que plusieurs établissements, qui dépendaient auparavant de différents ministères économiques ou de différentes grandes entreprises, ont été intégrés à l'Enseignement supérieur. Le besoin de recruter de nouveaux ingénieurs ne se faisant plus sentir dans les différentes branches économiques concernées par l'activité de ces établissements, il a paru ainsi utile de faire l'économie de l'entretien d'institutions de formation coûteuses, dont il était devenu impossible de garantir le recrutement de leurs diplômés. La mise sous la tutelle du ministère de l'Enseignement supérieur, a concerné ainsi par exemple I'Institut national d'électricité et d'électronique de Boumerdes, I'Institut national des matériaux de construction, I'Institut national des industries manufacturières, etc. Le placement de ces établissements sous la tutelle du mi nistère del'Enseignement supérieur, qui se traduit souvent par une diminution de l'efficacité pédagogique des formations dispensées, répondait, au demeurant, au besoin ressenti au niveau de ce ministère, de pouvoir disposer du grand nombre de places supplémentaires qu'exigeait l'augmentation constante du nombre de bacheliers. La « massification » atteignait ainsi ces établissements qui jusqu'alors avaient réussi à s'en préserver.

L'affaiblissement important, depuis plus de dix ans, de la position sociale des ingénieurs en Algérie ne constituet-il pas l'indice de l'échec d'une certaine vision scientiste de la modernisation, qui a prévalu depuis l'indépendance ? Les politiques inspirées par cette vision scientiste sont loin d'avoir réussi à atteindre leurs objectifs aussi bien économiques que culturels. La situation actuelle, caractérisée par un taux de nonemploi ou de déclassement important des ingénieurs et par une forte aspiration à l'émigration ne risque-t-elle pas d'inciter les gestionnaires du système éducatif à réduire fortement les effectifs des filières technologiques des établissements supérieurs, sans prendre en compte les manques à gagner qu'une telle politique produirait du point de vue de l'accumulation des savoirs modernes dans la société, comme du point de vue des aptitudes potentielles cette dernière à figurer en tant que sujet et non pas seulement en tant qu'objet dans les processus de mondialisation de l'économie? 


\section{N OTES}

1 L'étape de la graduation dans l'enseignement supérieur al gérien correspond aux cinq années d'études après le baccalauréat que nécessite l'obtention du diplôme d'ingénieur et aux quatre années conduisant à la licence, pour les autres disciplines.

2 Les données statistiques mentionnées sont puisées dans diverses publications du ministère de I'Enseignement supérieur et de la Recherche scientifique.

3 Les données recueillies à l'occasion du Recensement général de la population de 1998 devraient permettre de constituer des informations utiles sur la situation professionnelle d'une partie des ingénieurs résidant en Algérie. Le questionnaire utilisé pour ce recensement renseigne sur le dernier diplôme obtenu par les personnes concernées. Lecroisement de cetteinformation avec cellequi portesur les professions occupées par ces diplômés, et particulièrement les ingénieurs, donnerait la possibilité de mieux connaître la situation sociale de ces derniers.

4 LONGUENESSE Elisabeth et WAAST Roland, «Professions scientifiques en crise. Ingénieurs et médecins en Syrie, Egypte, Algérie», Revue Tiers M onde, t. 36, n 143, juillet-septembre 1995, pp. 489-490. 



\title{
Les ingénieurs de l'Institut algérien du pétrole : formation et parcours professionnels
}

\author{
Azzedine ALI BENALI
}

La politique d'industrialisation entreprise en Algérie dans les décennies 1960-1970 a permis à plusieurs générations de cadres, en particulier celles de l'après indépendance, de voir leur carrière progresser rapidement et d'accéder aux plus hautes fonctions de responsabilité L'Etat mobilisait alors des moyens techniques et financiers considérables pour la formation des ingénieurs et des techniciens, afin de garantir l'indépendance technique du pays. Aussi peut-on avancer l'idée que les ingénieurs et techniciens, en tant que nouveau «groupe socioprofessionnel », ont fait partie de l'élite qui a contribuéà créer et à gérer l'industrie publique algérienne. Bien que ces diplômés aient été formés par des universités ou des écoles, nationales ou étrangères, extrêmement diverses, ils possèdent des caractéristiques communes, intimement liées à I'histoire sociale de l'Algérie. Cependant, face à la crise et aux changements qu'a connus l'Al gérie dans la décennie 1990, le «corps » des ingénieurs tend à perdre sa rente de situation. Ce constat nous a conduit à nous intéresser, en particulier, aux devenirs des ingénieurs del'Institut al gérien du pétrole(IAP).

Jusqu'au milieu des années 1980, la plupart des ingénieurs pétroliers ont pu suivre des itinéraires professionnels et des trajectoires sociales répondant à leurs aspirations, du fait même de l'histoire du pétrole en A lgérie, de la spécificité de ce secteur économique et de leur formation très spécial isée. Créé en novembre 1965, trois ans après l'indépendance, I'IAP a été conçu par ses promoteurs comme un établissement de formation, de recherche et de développement des activités techniques et scientifiques, en relation avec les industries pétrolière, gazière, chimique et pétrochimique, et la production de matières plastiques, de moteurs et instruments divers. Situé à Boumerdes, à $50 \mathrm{~km}$ à l'est d'Alger, l'établissement dispose également des centres de formation de techniciens supérieurs à Arzew, Annaba, Skikda, Hassi R'mel et Hassi Messaoud. L'Institut algérien du pétrole a ainsi dispensé une formation de qualité, très poussée dans le domaine technique. 
Le caractère d'excellence des études à I'IAP (redoublement prohibé, moyenne de passage supérieur à la norme nationale, programme renforcé et très chargé, etc.) était vécu par les élèves ingénieurs comme un véritable fardeau. Toutefois, grâce à cette sélection, les lauréats de l'établissement ont pu valoriser le plus rapidement possible leur capital scolaire. Leurs attentes ont été favorisées par la politique de la Sonatrach. En effet, jusqu'à une époque récente, cette entreprise d'Etat géante qui monopolisait le secteur des hydrocarbures était également le principal employeur des ingénieurs diplômés de l'IAP.

Au regard de ces particularités propres à la figure de l'ingénieur pétrolier dans le système de formation-emploi, nous avons voulu savoir si le parcours professionnel des ingénieurs IAP est effectivement différent de celui des autres cadres techniques algériens : savoir comment le caractère spécifique de leur champ d'activité a pu faire évoluer leur carrière ; et comment, aujourd'hui, ils réagissent aux changements qui affectent le secteur pétrolier.

Ce texte analyse des sources documentaires issues de l'IAP et présente les premiers résultats d'une enquête conduite auprès d'un échantillon de 50 ingénieurs pétroliers, tous insérés dans le marché de l'emploi ${ }^{1}$. Un questionnaire fermé comprenait une trentaine de questions se rapportant tant au choix de leur formation qu'à leur trajectoire professionnelle ${ }^{2}$. Les réponses ainsi obtenues ont été complétées par des entretiens menés auprès de quelques ingénieurs de l'échantillon, choisis en fonction de leurs profils. Ces entretiens nous ont permis de connaître la manière dont certains ingénieurs de I'IAP se représentent les relations entre la formation reçue et leur parcours professionnel. Enfin, nous avons mené notre investigation aussi bien au sein de l'Institut que dans certaines entreprises employant les diplômés de l'Institut. Nous proposons donc, ici, de décrire le type de formation dispensée au sein de l'IAP, dans une perspective diachronique; puis les parcours professionnels des diplômés de l'Institut ; ainsi que les changements économiques et sociaux qui ont eu un impact sur leur stratégie d'insertion professionnelle.

\section{UN BINÔME FORMATION - EMPLOI EN ADÉQUATION ?}

De 1965 à 1999, I'Institut algérien du pétrole a formé 3172 ingénieurs (nationaux et étrangers) dans différentes spécialités ; et ses diplômés ont intégré, pour la plupart, différentes institutions pétrolières et gazières algériennes. Durant les vingt premières années, l'établissement a été placé sous la tutelle du ministère de l' Energie et des Industries chimiques et pétrochimiques. L'exercice de cette tutelle administrative signifiait que la formation des cadres et techniciens était subordonnée aux stratégies 
politiques élaborées par le ministère, en relation avec les différents plans de développement. L'IAP était aussi directement liéà I'Institut français du pétrole (IFP) qui, durant deux décennies environ, a assuré l'encadrement scientifique et technique, contribuant ainsi à la formation de nombreux ingénieurs algériens, en Algérie.

A partir de 1985 cependant, comme d'autres instituts de formation technologique, I'IAP passe sous la tutelle du ministère de l'Enseignement supérieur et de la Recherche scientifique. Plus récemment, en juin 1999, l'établissement est directement rattaché à la Sonatrach. L'ambition de cette grande société publique est de le transformer en une « grande école », d'envergure internationale, dans le domaine pétrolier. Aussi I'IAP essaie-t-il actuellement de mettre en application des méthodes de formation en adéquation avec les nouvelles normes et techniques internationales. Ces différentes réformes ont eu pour conséquence de multiplier les départements de spécialités directement liés à l'industrie pétrolière et à l'industrie gazière (cf. tableau 1$)^{3}$ : c'est dans le cadre de ces départements que sont dispensés les cours théoriques et pratiques, et organisés les examens et les stages. De fait, l'introduction de nouvelles spécialités répondait aux évolutions de l'économie pétrolière et de la politique d'industrialisation, visant plus particulièrement à contrôler le processus de production des hydrocarbures dans son ensemble, c'est-à-dire à couvrir toute la filière pétrolière en descendant vers l'aval. Ainsi, la formation a tout d'abord concernél'exploration et la prospection, puis la production et la transformation et, en dernier lieu, la maintenance des équipements pétroliers. En somme, entre 1966 et 1997, I'IAP a su répondre au développement des besoins du secteur pétrolier algérien dans le cadre d'une stratégie nationale d'indépendance dans le domaine des hydrocarbures.

Tableau 1. A nnées de création des départements de spécialités

\begin{tabular}{|c|c|}
\hline Spécialités & Années \\
\hline Raffinagepétrochimie & 1966 \\
\hline Géologie exploitation & 1967 \\
\hline Géophysique & 1968 \\
\hline Moteurs et applications & 1971 \\
\hline Gaz naturel liquéfié & 1974 \\
\hline Transport- Transport et distribution de gaz- production- forage & 1976 \\
\hline Plastiques & 1977 \\
\hline Chimie industrielle & 1982 \\
\hline Instrumentation & 1997 \\
\hline
\end{tabular}

Source : A nnuaire des ingénieurs d'Etat diplômés de l'Institut al gérien du pétrole (AIED-IAP), Alger 1999. 


\section{LES PRINCIPALES ÉTAPES DE L'HISTOIRE DE L'IAP}

\section{5-1976 : pallier la pénurie de cadres}

Durant une période fondatrice, dite «phase de récupération des richesses nationales », I'IAP a privilégié une formation étalée sur une courte période (2 à 3 ans), afin de pallier l'absence de cadres techniques nationaux dans le secteur très sensible des hydrocarbures. Entre 1966 et 1976, l'effectif des promotions annuelles des diplômés de I'Institut est passé de 4 à 122. Les nouveaux diplômés, dès leur sortie de l'établissement, étaient directement embauchés dans les entreprises publiques : ils ont ainsi collaboré, dans un premier temps, avec les ingénieurs coopérants, avant de les remplacer. Ces jeunes ingénieurs, spécialisés en géologie, géophysique ou en forage et production, avaient acquis un profil qui les destinait à la prospection et à la production pétrolière.

\section{EVOLUTION DE L'EFFECTIF DE L'IAP}

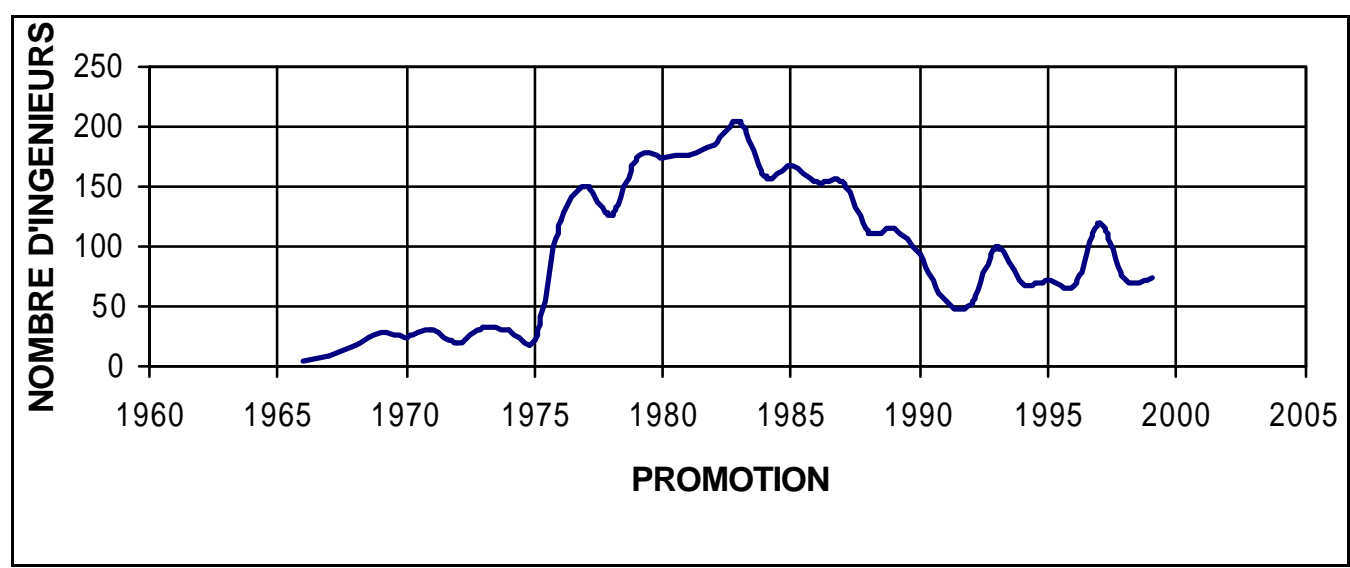

Source : A nnuaire des ingénieurs d'Etat diplômés de l'Institut Algérien du Pétrole (AIED-IAP), Alger 1999.

\section{6-1987 : I'instauration d'un double cursus}

La deuxième phase qui couvre la décennie 1976-1987 est une période de forte expansion des activités de I'IAP. Au cycle court vient s'adjoindre un cycle long de 5 ans qui est censé donner aux ingénieurs la maîtrise d'une part importante du processus de prospection, d'exploitation et de production du pétrole. Par ailleurs, c'est surtout durant cette période que les spécialités proposées par l'IAP s'accroissent et se diversifient. Quant au nombre de diplômés par promotion, il augmente chaque année pour atteindre un pic en 1983 (204). Cette croissance des effectifs nous paraît être une conséquence directe de la hausse des prix du pétrole (1977-1983) à l'échelle internationale. L'augmentation des 
revenus tirés de la vente du pétrole a contribué en effet au développement du secteur des hydrocarbures algériens qui, pour faire fonctionner ses nouvelles installations, a fait appel à une main-d'œuvre qualifiée de plus en plus nombreuse.

\section{8-1999 : les ingénieurs de la crise}

Le retournement de la conjoncture pétrolière, à partir du milieu des années 1980, aurait, de la même façon, entraîné la mise en œuvre d'un train de réformes au sein de I'IAP. L'Institut propose deux nouvelles spécialités à ses élèves, la chimie industrielle et I'instrumentation. Mais c'est aussi durant cette décennie que le nombre d'ingénieurs formés chaque année tend à diminuer. Aux difficultés économiques s'ajouterait la saturation du marché de l'emploi pour les ingénieurs du secteur des hydrocarbures, désormais incapable d'absorber les lauréats de I'IAP. Aussi l'établissement doit-il modifier sa stratégie de formation en éliminant sa filière courte, provoquant de ce fait une diminution considérable des effectifs formés chaque année. La taille des promotions atteint son niveau le plus bas en 1992, avec seulement 54 diplômés. Par la suite, elle s'accroît quelque peu pour donner environ 70 ingénieurs par an (avec un pic de 120 en 1997) 4 .

Tableau 2. Distribution des effectifs des ingénieurs diplômés de I'IAP en fonction du cycle de formation

\begin{tabular}{l|c|c|}
\hline \multicolumn{1}{c|}{ CYCLE DE FORMATION } & EFFECTIF & \% \\
\hline CYCLE LONG & 2371 & 76,78 \\
\hline CYCLE COURT & 717 & 23,21 \\
\hline TOTAL & $\mathbf{3 0 8 8}$ & $\mathbf{1 0 0}$ \\
\hline
\end{tabular}

Source : A nnuaire des ingénieurs d'Etat diplômés de l'Institut algérien du pétrole (AIED-IAP), Alger 1999.

\section{L'ÉVOLUTION DES MODALITÉS D'ACCÈS À L'IAP}

On vient de voir que les modalités d'accès à I'IAP ont été aussi bien déterminées par la politique industrielle de l'Algérie que par ses transformations économiques et sociales. Au cours des années 1960, le faible nombre de bacheliers al gériens conduit I'IAP à recruter des candidats titulaires d'un brevet d'enseignement moyen ou d'un certificat d'aptitude professionnel (CAP), ayant eu une formation en entreprise sanctionnée par un diplôme technique. Ces élèves suivent alors le cycle court de l'Institut, en vue d'obtenir 
un diplôme d'ingénieur. Dès les années 1970 cependant, l'entrée à l'Institut est plus sélective. Désormais, I'IAP recrute sur concours les élèves du niveau «terminale » des cycles «techniques mathématiques » et «mathématiques et scientifiques ». Quant aux bacheliers de ces mêmes cycles, ils peuvent s'inscrire automatiquement, sans sélection préalable. Cette tendance à la sélection se confirme dans la décennie 1980, puisque désormais seuls les titulaires des baccalauréats scientifiques et techniques sont admis.

En ce qui concerne la filière longue, les étudiants suivent d'abord un tronc commun d'un an (de deux ans depuis 1996), dans lequel sont dispensés un enseignement général scientifique et technique, ainsi qu'une formation en sciences sociales. Ensuite, les étudiants sont orientés vers tel ou tel enseignement de spécialité, en fonction de leurs vœux et surtout de la moyenne obtenue durant l'année du tronc commun. Outre cette formation théorique, l'élève ingénieur suit des enseignements pratiques, liés à sa spécialité, dans les nombreux laboratoires de l'Institut ou sur les sites pétroliers (Hassi Rmel, Hassi Messaoud, Arzew, etc.). Au terme de la cinquième année, l'étudiant doit élaborer un projet de fin d'étude, puis rédiger un mémoire. Ce dernier est soutenu devant un jury, présidé par un représentant du secteur industriel concerné par le sujet, composé d'enseignants de l'IAP et de personnalités du monde de l'entreprise.

La formation de l'IAP reste d'un niveau élevé, mais elle est désormais encore plus sélective, dans la mesure où le redoublement est interdit, et le passage aux classes supérieures conditionné à l'obtention d'une moyenne de 12 sur 20. Néanmoins, cette formation donne une maîtrise effective et approfondie des techniques et des méthodes de travail, grâce notamment à l'intervention d'experts et de professionnels du domaine pétrolier.

\section{LA NOUVELLE SATURATION DU MARCHÉ DE L'EMPLOI}

Jusqu'au milieu de la décennie 1980, la plupart des ingénieurs diplômés de I'IAP se sont insérés dans le monde du travail par le biais d'accords passés entre l'IAP et le ministère de l' Energie. Les procédures d'embauche se réduisaient à un simple et court entretien de motivation entre le candidat et le recruteur. A l'issue de cet entretien, le candidat était immédiatement admis dans un bureau d'étude pour une courte durée, et ensuite affecté à un poste de façon définitive. Mais alors que I'Institut continuait, jusqu'en 1987, à former des promotions de plus de 150 ingénieurs pétroliers, la crise économique - consécutive à la baisse du prix du pétrole - portait un coup d'arrêt aux 
investissements dans de grands projets industriels. Le secteur des hydrocarbures n'absorbant plus tous les nouveaux diplômés, certains ingénieurs pétroliers se sont retrouvés au chômage.

Ces difficultés d'insertion ont contribué à réactiver des réseaux informels de recrutement. Les entreprises publiques embauchent toujours, de façon officielle, en publiant des offres d'emploi dans la presse. Mais les critères de sélection des candidats relèvent surtout de mobilisations familiales ou régionales. Les détenteurs d'un capital relationnel important sont ceux qui accèdent le plus facilement à un emploi dans les entreprises du secteur des hydrocarbures. L'accentuation de la crise dans les années 1990 et l'arrêt officiel, par décret présidentiel, du recrutement des sociétés publiques ont débouché sur la généralisation de pratiques informelles d'embauche.

\section{LES FONCTIONS DES INGÉNIEURS DIPLÔMÉS DE L'IAP}

L'exploitation des données de l'annuaire des ingénieurs de l'IAP montre que les fonctions exercées par ces derniers recouvrent aussi bien les domaines technique et administratif que le champ de l'enseignement et de la recherche.

\section{Les fonctions techniques}

Plus de la moitié des ingénieurs diplômés IAP (soit 57 \%) occupent des fonctions techniques. Dans ce domaine, on trouve principalement de jeunes ingénieurs qui cherchent à acquérir compétence et expérience dans la maîtrise du processus de production. Le passage du jeune ingénieur pétrolier de l'IAP pendant quelques années dans les ateliers ou les unités pétrolières du Sud algérien est quasiment obligatoire. Travailler au Sahara, dans les zones d'exploitation des hydrocarbures, permet à I'ingénieur pétrolier de maîtriser toutes les dimensions techniques de son travail. II peut ai nsi parfaire ses connaissances dans l'exploration pétrolière, le forage, l'exploitation et la distribution du pétrole, etc.

Le fait que le secteur des hydrocarbures soit un monopole d'Etat explique que la plupart des ingénieurs de I'IAP occupent des fonctions techniques au sein de grandes entreprises pétrolières et gazières (Sonatrach, Sonelgaz, GTP, Naftal, EN PC, EN EP, etc.). Mais force est de constater que la majorité des diplômés de l'Institut sont employés par la Sonatrach. Travailler pour cette entreprise est très recherché dans la mesure où elle propose des conditions matérielles particulièrement avantageuses. 


\section{Les fonctions administratives}

Plus d'un quart del'effectif des ingénieurs diplômés del'IAP (soit 27 \%) occupe des fonctions administratives. La plupart d'entre eux ont obtenu leur diplôme dans les années 1970 et 1980. Ils constituent un groupe d'ingénieurs privilégiés chargé de la gestion et de la direction des départements administratifs des sociétés pétrolières ou des divers établissements publics. Ainsi, le développement de la carrière s'accompagne de l'abandon des fonctions techniques exercées dans les complexes et installations pétrolières du Sud au profit de fonctions managériales plus en rapport avec les motivations et ambitions personnelles des ingénieurs IAP. L'exercice de responsabilités administratives signifie pour ces derniers un retour vers les centres urbains du nord de l'A Igérie ainsi qu'un rapprochement avec la famille restée sur place.

Certains occupent de hautes fonctions au sein de la hiérarchie des sociétés publiques : en 1999, on compte neuf PDG des plus importantes entreprises pétrolières et gazières al gériennes (Sonatrach, GTP, NAFTOGAZ, ENSP, ENTP, EN EL, ENAFOR, etc.). Les ingénieurs de I'IAP exercent pour la plupart des fonctions de responsabilité importantes : les postes de direction de haut niveau (du sous-directeur au PDG) représentent plus du tiers des effectifs (cf. tableau 3).

Tableau 3. D istribution des ingénieurs de l'IA P selon l'exercice d'une fonction de responsabilité

\begin{tabular}{|l|c|c|}
\hline \multicolumn{1}{|c|}{ POSTE } & EFFECTIF & $\%$ \\
P.D.G & 9 & 3 \\
\hline D.G & 15 & 4 \\
\hline DIRECTEUR & 94 & 26 \\
\hline SOUS DIRECTEUR & 8 & 2 \\
\hline CHEF DEPARTEMENT & 43 & 12 \\
\hline CHEF DE SERVICE & 102 & 29 \\
\hline AUTRES & 84 & 24 \\
\hline TOTAL & $\mathbf{3 5 5}$ & $\mathbf{1 0 0}$ \\
\hline
\end{tabular}

Source: Annuaire des ingénieurs d'E tat diplômés de l'Institut al gérien du pétrole (AIED -IAP), A Iger 1999.

\section{Enseignement - recherche et formation}

Sur un total de 1329 diplômés de l'IAP, seule une minorité (211 soit 16 \%) occupe des fonctions en relation avec la formation, la recherche et l'enseignement. Certains ont pu compléter leur formation à l'étranger, en Angleterre, au Canada, en France ou aux 
Etats-Unis. En Algérie, ces ingénieurs exercent leurs activités dans les divers établissements d'enseignement supérieur, tels que l'université des sciences et de la technologie Houari Boumedienne (USTHB), I'université d'Oran, I'IAP et I'Institut national d'électronique (INELEC). Ils sont chercheurs ou enseignants dans des spécialités comme les plastiques (64), la chimie industrielle (32), le raffinage et la pétrochimie (22), ou encore administrateurs (chefs de départements ou directeurs d'instituts). L'essentiel des ingénieurs exerçant dans l'enseignement et la recherche sont très attachés à la val eur symbolique du savoir scientifique : ils vivent leur travail comme un sacerdoce. Cet attachement compense le fait qu'ils ont moins de responsabilités que les ingénieurs employés dans les sociétés publiques.

\section{LES CARACTÉRISTIQUES SOCIOÉCONOMIQUES DES INGÉNIEURS IAP}

Nous allons étudier dans cette partie certaines caractéristiques spécifiques de notre échantillon d'enquête.

Tableau 4. Distribution des ingénieurs de l'IA P selon le sexe

\begin{tabular}{lc|c|}
\hline Sexe & Effectifs & $\%$ \\
Hommes & 48 & 96 \\
\hline Femmes & 2 & 4 \\
\hline Total & $\mathbf{5 0}$ & $\mathbf{1 0 0}$
\end{tabular}

Source: Enquête AzzedineAli Benali, mars-mai 2000.

Les femmes sont quasiment absentes parmi les diplômés de l'échantillon. Si l'on prend les données de l'annuaire des ingénieurs de l'IAP de 1999, on constate une légère sous-représentation des femmes issues de I'IAP (7 \%) par rapport à la population féminine totale, titulaire d'un diplôme d'ingénieur. En effet, sel on le recensement de 1989, elles représentent $8 \%$ del'ensemble de la population ingénieur. En outre, elles travaillent exclusivement dans l'enseignement et la recherche, et n'exercent pas de responsabilités particulières: les femmes ingénieurs sont principalement employées dans les laboratoires qui se concentrent dans le nord de l'A Igérie. Justement, cette légère sous-représentation des femmes ingénieurs dans le secteur pétrolier pourrait s'expliquer par le passage obligé des jeunes diplômés de l'IAP par les complexes et installations pétrolières du Sud. II est impensable, dans la société al gérienne contemporaine, que les femmes partent travailler sur les lieux de production des hydrocarbures, loin de leur famille. 
Tableau 5. D istribution des ingénieurs de l'IAP selon la spécialité

\begin{tabular}{|l|c|c|}
\hline \multicolumn{1}{|c|}{ Spécialités } & Effectifs & \% \\
Forage & 19 & 38 \\
Géologie & 5 & 10 \\
\hline Géophysique & 11 & 22 \\
\hline Gaz naturel liquéfié (GNL) & 2 & 4 \\
Raffinage et pétrochimie & 2 & 4 \\
\hline Autres & 11 & 22 \\
\hline Total & $\mathbf{5 0}$ & $\mathbf{1 0 0}$ \\
\hline
\end{tabular}

Source: Enquête Azzedine Ali Benali, mars-mai 2000

$70 \%$ des ingénieurs de l'échantillon sont spécialisés dans le forage, la géologie et la géophysique. Cela signifie que les diplômés de l'IAP seconcentrent dans des spécialités en relation avec la production et l'exploitation. Cette situation est très certainement imputable au fait que ces deux domaines se situent au cœur de la stratégie pétrolière al gérienne.

\section{Tableau 6. D istribution des ingénieurs de I'IAP selon la taille de l'entreprise}

\begin{tabular}{l|c|c}
\hline \multicolumn{1}{c|}{ Taille de l'entreprise } & Effectif & \% \\
Moins de 50 personnes & - & - \\
De 50-99 personnes & - & - \\
De 100-499 personnes & 6 & 12 \\
\hline De 500-999 personnes & 1 & 2 \\
\hline De 1 000-9 999 personnes & 15 & 30 \\
\hline Plus de 10 000 personnes & 28 & 56 \\
\hline Total & $\mathbf{5 0}$ & $\mathbf{1 0 0}$ \\
\hline
\end{tabular}

Source: Enquête AzzedineAli Benali, mars-mai 2000.

Nous remarquons que notre population se retrouve logiquement dans de grandes entreprises. Les ingénieurs sont massivement employés par des sociétés de plus de 1000 personnes (86\%). En effet, les entreprises du secteur des hydrocarbures sont de grande taille et exigent la présence d'une main-d'œuvre qualifiée.

Le parcours professionnel des ingénieurs de l'Institut algérien du pétrole se caractérise par une forte mobilité intra-entreprise ; ce type de mobilité interne est prédominant par rapport à la mobilitéinter-entreprises. 
Tableau 7. D istribution des ingénieurs selon qu'ils ont ou non connu une période d'inactivité

\begin{tabular}{l|cc|}
\hline \multicolumn{1}{c|}{ Réponse } & Effectif & $\mathbf{\%}$ \\
\hline $\begin{array}{l}\text { Ingénieurs ayant connu une période de recherche } \\
\text { d'emploi }\end{array}$ & 11 & 22 \\
$\begin{array}{l}\text { Ingénieurs n'ayant pas connu une période de recherche } \\
\text { d'emploi }\end{array}$ & 39 & 78 \\
\hline Total & $\mathbf{5 0}$ & $\mathbf{1 0 0}$ \\
\hline
\end{tabular}

Source : Enquête Azzedine Ali Benali, mars-mai 2000.

A partir de ce tableau, il apparaît que plus des trois quarts des ingénieurs (78\%) n'ont pas connu de période de recherche d'emploi ou de chômage. Ces résultats nous montrent avec quelle facilité les ingénieurs IAP se sont insérés dans la vie active (tout au moins jusqu'à la fin des années 1980).

\section{Tableau 8. Distribution des ingénieurs de l'IAP selon la façon d'obtenir un premier emploi}

\begin{tabular}{lll}
\hline \multicolumn{1}{c}{ Obtention du premier emploi } & Effectif & \% \\
\hline Par annonce & 4 & 8 \\
\hline Réseau personnel & 2 & 4 \\
Candidature spontanée & 18 & 36 \\
A travers les conventions signées & 26 & 52 \\
Total & $\mathbf{5 0}$ & $\mathbf{1 0 0}$ \\
\hline
\end{tabular}

Source : Enquête AzzedineAli Benali, mars-mai 2000.

Les réponses des ingénieurs sur leur premier emploi confirment le constat établi après analyse du tableau précédent. N otre population a bénéficié, grâce aux conventions signées entre I'IAP et les diverses entreprises publiques, d'une insertion rapide dans la vie active.

Tableau 9. Distribution des ingénieurs de l'IAP selon leur mobilité professionnelle

\begin{tabular}{l|cc|}
\hline Changement d'entreprise & Effectif & $\%$ \\
Ont changé d'entreprise & 14 & 28 \\
\hline N'ont pas changé d'entreprise & 36 & 72 \\
\hline Total & $\mathbf{5 0}$ & $\mathbf{1 0 0}$
\end{tabular}

Source : Enquête Azzedine Ali Benali, mars-mai 2000. 
N ous remarquons, d'après le tableau 9, que $72 \%$ des ingénieurs n'ont pas changé d'entreprise. Ce qui confirme la faible mobilité inter-entreprises des diplômés de I'IAP. Les ingénieurs ne ressentent pas le besoin de changer d'employeur dans la mesure où, employés dans de grandes entreprises pétrolières, ils bénéficient de nombreux avantages matériels et privilèges par rapport aux sociétés d'autres secteurs del'industrie al gérienne. En revanche, le pourcentage des ingénieurs de I'IAP qui ont occupé plus d'une fonction est de $62 \%$. La mobilité des ingénieurs concerne bien plus le passage d'une fonction à une autre au sein d'une même société que le changement d'entreprise.

Tableau 10. Relation entre la fonction de responsabilité occupée actuellement et la mobilité

\begin{tabular}{|c|c|c|c|c|c|c|c|}
\hline Poste & Ingénieur & $\begin{array}{l}\text { Chef de } \\
\text { service }\end{array}$ & $\begin{array}{c}\text { Chef de } \\
\text { département }\end{array}$ & $\begin{array}{c}\text { Sous- } \\
\text { directeur }\end{array}$ & Directeur & Autres & Total \\
\hline \multirow{2}{*}{$\begin{array}{l}\text { Mobilité } \\
\text { Ont changé } \\
\text { d'entreprise }\end{array}$} & 3 & 3 & 2 & 1 & 1 & 4 & 14 \\
\hline & $11 \%$ & $37,50 \%$ & $40 \%$ & $50 \%$ & $33,33 \%$ & $80 \%$ & $28 \%$ \\
\hline \multirow{2}{*}{$\begin{array}{l}\text { N'ont pas changé } \\
\text { d'entreprise }\end{array}$} & 24 & 5 & 3 & 1 & 2 & 1 & 36 \\
\hline & $88,88 \%$ & $62,50 \%$ & $60 \%$ & $50 \%$ & $66,66 \%$ & $20 \%$ & $72 \%$ \\
\hline \multirow[t]{2}{*}{ Total } & 27 & 8 & 5 & 2 & 3 & 5 & 50 \\
\hline & $100 \%$ & $100 \%$ & $100 \%$ & $100 \%$ & $100 \%$ & $100 \%$ & $100 \%$ \\
\hline
\end{tabular}

Source: Enquête AzzedineAli Benali, mars-mai 2000.

Le tableau 10 montre la relation existant entre la fonction de responsabilité occupée et la mobilité des ingénieurs de l'IAP. D'après les premiers résultats, nous constatons que ceux qui n'ont pas changé d'entreprise sont également ceux qui n'ont pas obtenu de poste hiérarchique supérieur. En revanche, ceux qui ont changé d'entreprise, pendant leur trajectoire professionnelle, sont ceux qui occupent des fonctions de responsabilité supérieure, administrative ou de gestion. Il apparaît ainsi que les ingénieurs de l'IAP sont en position d'attente : vraisemblablement plus que d'autres ingénieurs, ils recherchent une promotion au sein de la grande entreprise publique qui les emploie. En effet, leur formation hautement spécialisée, ai nsi que la spécificité de leur poste, ne les prédisposent pas à une grande mobilité d'un secteur économique à l'autre.

Au terme de cette première enquête, nous pouvons avancer certains résultats. Tout d'abord, l'itinéraire professionnel de l'ingénieur issu de l'Institut algérien du pétrole est caractérisé par une forte stabilité dans l'entreprise d'origine. 
Des différences existent bien entre les générations d'ingénieurs pétroliers al gériens issus de I'IAP. Certains ont pu bénéficier de nombreux avantages et situations. Nombreux sont ceux, parmi la dernière génération de diplômés, qui subissent les effets de la crise, à l'instar des autres catégories socioprofessionnelles.

Les ingénieurs pétroliers issus de I'IAP exercent des responsabilités dans le domaine technique. II nous est apparu qu'ils sont attachés à leur fonction, et que I'intensité du rythme de leur formation, ainsi que le capital théorique et technique acquis pendant cinq ans à l'Institut, les a certainement rendus plus aptes à rester dans le domaine pétrolier sans quitter l'entreprise.

Une des caractéristiques des ingénieurs de I'IAP est une forte mobilité dans I'entreprise. Si la majorité des ingénieurs reste stable au sein de leur entreprise d'origine, nous remarquons un mouvement ascendant dans les postes hiérarchiques durant leur trajectoire professionnelle. En revanche, le changement d'entreprise pour une grande majorité d'ingénieurs IAP signifie la construction d'une vie professionnelle nouvelle.

L'élargissement de l'étude à cette catégorie d'ingénieurs algériens, formés à I'Institut français du pétrole, en vue d'une éventuelle comparaison, pour une même population issue de deux instituts différents, pourra permettre de mieux saisir l'apport de la formation dans l'évolution d'une carrière.

\section{N OTES}

1 Nous avons effectué une préenquête pour identifier les sources documentaires et construire l'échantillon.

2L'échantillon comprend des ingénieurs pétroliers des deux sexes, de diverses promotions, mais avec au minimum cinq ans d'expérience et exerçant des fonctions techniques, administratives où travaillant dans l'enseignement et la recherche.

3 On compte neuf départements spécialisés dans les domaines suivants : Géologie, Géophysique, Exploitation, Instrumentation, Chimie Industrielle, Pétrochimie, Gaz, Plastiques, Moteurs et applications. L'IAP est admi nistré par une direction générale, assistée d'un conseil de direction et d'un conseil scientifique disposant d'une large autonomie. Ce dernier coordonne et contrôle les activités de l'Ecole d'ingénieurs de Boumerdes, ainsi que les centres de techniques appliquées. long.

4 II convient également designaler que 75 \% des ingénieurs diplômés de l'IAP sont passés par le cycle 

CHAPITRE 2

\section{FILIÈRES COLONIALES ET PRATIQUES PROFESSIONNELLES}





\title{
Figures d'ingénieurs pendant le Protectorat français en Tunisie : l'exemple de la Poste et des Travaux publics
}

\author{
Habib BELAÏD
}

La question de la formation d'une élite économique ou technique dans la Tunisie coloniale est encore à l'ordre du jour. En effet, l'élite tunisienne moderne est essentiellement composée d'avocats et de médecins qui ont joué pendant l'époque coloniale un rôle social et politique de premier plan. En revanche, l'élite technique est quasiment absente. L'historiographie tunisienne a négligé jusquelà I'histoire des techniques et de leur application, comme élément relevant avant tout du «domaine colonial ». La figure de l'ingénieur colonial ne fait-elle pas partie de ce champ d'investigation ? ${ }^{1}$

Dans le cadred'un débat plus large, nous avons poséla question suivante: pourquoi la société tunisienne a-t-elle été longtemps réfractaire à l'introduction d'une culture technique moderne issue de l'industrialisation ? La domination coloniale est-elle seule en cause ? La formation d'ingénieurs suffit-elle pour accéder à cette culture technique ?²

Nous partons du constat que, à part quelques individualités, la présence des ingénieurs et techniciens tunisiens dans le corps des ingénieurs de Tunisie est minime. Vers 1900, époque des grands travaux effectués en Tunisie par les autorités du Protectorat, le personnel tunisien des Travaux publics ne représentait que $5 \%$ du total du personnel permanent et temporaire 3 ; plus tard, en 1945, la section tunisienne del'Union des ingénieurs et techniciens (UNITEC) déplore dans son bulletin la très faible présence de techniciens tunisiens dans cet organisme.

Les travaux de Lilia Ben Salem 4 expliquent en partie les raisons de la nonémergence d'un corps d'ingénieurs tunisiens dans le contexte colonial. Les principales sont à rechercher dans la nature de l'enseignement dispensé sous le Protectorat et dans la politique coloniale elle-même qui s'opposait à un enseignement technique de haut 
niveau. Partant du fait que « les fonctions techniques sont des fonctions d'encadrement professionnel, et donc social », Hocine Khelfaoui constate ${ }^{5}$ que le « colonialisme voulait éviter à tout prix d'avoir à assurer l'encadrement des indigènes par des indigènes ». Des raisons sociales expliquent aussi cette carence: les ingénieurs naissent ou accompagnent l'industrialisation d'un pays. Or, à l'époque du Protectorat, la sociététunisiennen'accède pas à ce type de développement.

Afin de vérifier ces assertions, nous nous proposons d'étudier deux institutions relativement anciennes et, en même temps, stratégiques : la Poste et les Travaux publics. La première remonte à une époqueantérieure à l'établissement du Protectorat en Tunisie. Dès 1847, est installé un système de communication à distance (télégraphe Chappe), suivi, à partir de 1860-1861, de l'installation de la télégraphie électrique, en application d'une convention tuniso-française (1859). Or celle-ci prévoyait entre autres stipulations la formation de techniciens tunisiens. L'Office postal tunisien, créé en 1888, prit en charge par la suite l'installation et la gestion des services postaux, du télégraphe et du téléphone (1895). Pour des raisons politiques et militaires, cet Office postal est alors créé «en dehors » de l'administration tunisienne ${ }^{6}$. Quant à la direction des Travaux publics, elle est instituée quel ques mois seulement après l'établissement du Protectorat en Tunisie par décret le 3 septembre 1882. Cette institution mérite qu'on s'y intéresse aussi bien par la dimension de son champ d'intervention que par le personnel affecté à ses travaux.

Sans prétendre à l'exhaustivité, nous privilégierons trois points qui nous paraissent essentiels : l'évolution comparée des deux institutions, à travers le rôle imparti à leurs membres les plus actifs ; la vie associative des ingénieurs telle qu'elle a pu être reconstituée grâce à l'établissement d'un corpus ; enfin les itinéraires individuels, indicateurs de changement ou de rupture.

\section{À LA RECHERCHE D'Un CORPUS D'INGÉNIEUR}

Une enquête rétrospective sur les ingénieurs en Tunisie se fonde sur des sources officielles ${ }^{7}$, complétées par les annuaires, bulletins, livres d'or, presse, etc., et par quelques témoignages oraux. II s'agit d'abord d'identifier le ou les groupe(s) et de constituer un corpus pour tenter une sociologie d'un corps nécessairement hétérogène. Cette démarche devrait nous permettre de déceler, dans les rapports entre des groupes de statuts différents, les conflits ou frictions qui surgissent entre ingénieurs français métropolitains et locaux d'une part, et entre ingénieurs français et ingénieurs et techniciens tunisiens d'autre part. 
Peut-on identifier des générations d'ingénieurs dans le cas des ingénieurs français ? On sait que les bâtisseurs des premiers temps de la colonisation (surestimés par le mythe des « pionniers »), tels que Paul Michaud et Georges Paviller pour les Travaux publics, ont disposé de pouvoirs étendus: qu'ils sont suivis par les gestionnaires dansl'entre-deuxguerres, puis par les techniciens dans l'après-guerre et au cours des années 1950. Mais dans quelle mesure ce schéma est-il valable?

Le projet de formation des techniciens et des ingénieurs remonte en Tunisie au milieu du XIX siècle, lorsque le réformateur A hmed Bey a créé l'Ecole militaire du Bardo (1840) ; mais il s'agit, comme dans le reste de l'Empire ottoman, d'ingénieurs militaires. L'absence de croissance industrielle à l'instar de l'Europe a été l'une des principales causes de l'échec de ce projet. Cependant, certains grands travaux engagés par les beys ont nécessitéla constitution d'un noyau de corps d'ingénieurs d'Etat (français en général) opérant auprès du pouvoir beylical ${ }^{8}$. La colonisation va non seulement couper court à tout projet d'industrialisation du pays en créant « le pacte colonial », mais la métropole fournira des ingénieurs à la colonie, affirmant ainsi sa domination technique.

Le développement des Travaux publics et de la Poste a nécessitél'engagement d'un personnel important, comme en témoignent ces quelques indications : dans les deux cas, le détachement (pour 5 ans) d'ingénieurs et techniciens français est fréquent jusqu'aux années 1920 ; le recours aux Français en contrat local a nécessité de la part de l'administration coloniale une réadaptation des statuts et leur alignement sur ceux de la métropole. Dans le mode de recrutement (détachement à partir de la métropole, ou recrutement local), nous pensons qu'il a pu exister dans le mouvement (ou rotation) des ingénieurs entre métropole et colonie (et inversement) des stratégies de carrières pour les ingénieurs français en Tunisie, mais sans que cela devienne la règle ${ }^{9}$.

\section{Le secteur des postes ${ }^{10}$}

La mise en place de l'infrastructure des Postes a nécessité un personnel qualifié : en 1891, l'ingénieur des Ponts et Chaussées Resal a été chargé de la construction de I'Hôtel des Postes à Tunis ; Sins, ingénieur de l'Office postal, a dirigé les travaux du réseau téléphonique ${ }^{11}$. Par ailleurs, les effectifs des postiers - français essentiellement sont en progression constante jusqu'en 1933. Si un fléchissement relatif se dessine à partir de 1934, apparemment la crise n'a pas affecté cette progression. En fait, le «nivellement » s'est fait par le haut : les fonctions de directeur des services postaux et financiers et celles des chefs de service sont supprimées ; I'emploi de directeur général ${ }^{11}$ 
est remplacé par un fonctionnaire ayant grade de directeur départemental ou régional de moindre importance. Quant au poste d'ingénieur ou d'inspecteur breveté, créé lors des travaux de la Commission Tirmann (1924-1925) il ne sera jamais pourvu. Dans I'entre-deux-guerres, l'ingénieur se fait encore rare : en 1923, l'organigramme de l'Office postal n'en comporte qu'un seul, le chef du service technique ; poste qui sera pourvu en 1937 par un sous-ingénieur (Clément). L'installation en 1938 d'une radio de diffusion d'Etat (Radio-PTT devenu Radio-Tunis) s'effectue sous la conduite de deux ingénieurs des PTT : I'un venant de France en mission (Godfin), I'autre, Jean Cabantous, est chef de service technique de I'Office postal tunisien ${ }^{13}$. En 1949, la gestion technique des différentes installations de l'Office aurait nécessité plus de personnel qualifié. Quatre ingénieurs sont alors employés par l'Office: un chef de service (René Le Gourrierec), un responsable des câbles souterrains (Bergeron), un responsable du service Radio (Courvoisier) et un responsable des Transports, Mohamed Mili. Ce dernier est le seul ingénieur tunisien dans le secteur. L'augmentation du nombre d'inspecteurs est également significative de cette évolution : en 1936, on ne comptait que 2 inspecteurs et 12 rédacteurs (dont certains « faisant fonction » d'inspecteurs), al ors que 25 inspecteurs sont en activité en 1950.

La Seconde Guerre mondiale avait, en partie, favorisél'entrée des agents tunisiens à I'Office postal en remplacement des Français mobilisés. Secteur névralgique par excellence, où les communications faisaient partie de la stratégie militaire, l'Office postal avait été touché à différents niveaux par la guerre. Outre les bâtiments endommagés, le personnel lui-même avait été affecté en 1944 par la mobilisation de 284 fonctionnaires (agents permanents ou temporaires) en particulier d'agents contrôleurs des installations, électromécaniciens, surveillants des dérangements, agents de lignes (4). Pour des raisons « stratégiques », aucune formation d'ingénieurs tunisiens n'avait été envisagée à court ou long terme.

\section{Les ingénieurs des Travaux publics : physionomie d'un corps}

La direction des Travaux publics (TP) en Tunisie détenait des compétences très larges, à l'instar de la direction des Finances. En effet, cette Direction générale était chargée de presque tous les services techniques du pays. Son directeur avait rang de ministre du Bey sans autre tutelle ministérielle. Cette position exceptionnelle permet de mesurer l'importance des ingénieurs sur le plan technique, aussi bien que politique. Mais l'absence d'études économiques et techniques portant sur le secteur des Travaux publics 
nous empêche d'avoir une vue globale sur le corps des ingénieurs en Tunisie et sur son évolution. Nous partons cependant de quelques données éparses pour essayer de reconstituer ce corps.

En 1936, l'organigramme de la direction des Travaux publics fait état de 59 ingénieurs (tous français) de différents services et grades. Leur répartition par secteur est la suivante : outre l'ingénieur chef du cabinet du directeur des TP, 11 ingénieurs sont responsables des mines, 3 du contrôle des chemins de fer et des transports automobiles, 4 du service central des routes, phares et balises (incluant les ports maritimes et aériens, et la distribution de l'énergie électrique) ; 5 ingénieurs sont affectés au service hydraulique, travaux communaux, etc., 13 au service topographique ${ }^{14}, 17$ à la Compagnie fermière des chemins de fer tunisiens et 5 au service électrique de la Compagnie du gaz et des eaux de Tunis ${ }^{15}$.

Dans l'immédiat après-guerre, les problèmes de la reconstruction, la recherche de nouvelles formes d'énergie ${ }^{16}$, entre autres, ont nécessité l'emploi d'un nombre accru d'ingénieurs et de techniciens. La majeure partie d'entre eux vient de la métropole, avec parfois un passage par l'A lgérie, et leur statut prend la forme d'un détachement de 5 ans renouvelables. La part des personnels locaux français est infime et plus encore celle des Tunisiens ${ }^{17}$. Le recrutement des ingénieurs évolue presque en cercle fermé parmi les diplômés des grandes écoles françaises, mais les directeurs des Travaux publics sont en général choisis parmi ceux qui ont exercé longtemps en Tunisie (par exemple, Gosselin dans I'hydraulique agricole en Tunisie depuis 1922, ou J-L. Bonnenfant depuis 1932). Compte tenu de la situation économique du pays, l'expérience et l'initiative personnelles comptent beaucoup plus qu'en France, notamment pour accéder à des postes de responsabilité régionales ou nationales. L'expérience « tunisienne » est vivement souhaitée pour ces responsabilités.

\section{La prédominance du corps des Ponts et Chaussées}

Sur les 13 ingénieurs des Travaux publics de l'Etat (TPE) proposés pour le renouvellement de leur détachement en Tunisie (en 1946), 12 sont des ingénieurs TPE, venus des Ponts et chaussées, un seul des Mines. Les ingénieurs des Ponts et Chaussées, toutes catégories confondues (quatre classes) prédominent donc dans cette direction, alors que dans l'administration de la Poste, on trouve des polytechniciens, tels que Jean Dezès, directeur de l'Office postal de Tunisie, après des études à l'Ecole nationale supérieure de télécommunications. 
Une liste de 1949 confirme cette prédominance du corps des Ponts et Chaussées au détriment de celui des Mines : "Sur les 6 ingénieurs en chef $(. .$.$) en fonction,$ 3 appartiennent au corps des Ponts et Chaussées et 1 au corps des Mines. L'ingénieur en chef des transports est un polytechnicien provenant de la SNCF. L'ingénieur en chef du service des Aménagements urbains et du service topographique est un Tunisien, ancien élève de l'Ecole polytechnique à titre étranger. Sur les 7 ingénieurs principaux, 6 appartiennent au corps des Ponts et Chaussées et 1 aux Mines $»^{18}$. Les ingénieurs des Travaux publics du service des Ponts et Chaussées se recrutent en majorité parmi les ingénieurs des Travaux publics de l'Etat du cadre métropolitain. Pour le cadre français local, les possibilités de recrutement sont soit le concours public (avec le même programme que celui d'ingénieur TPE dans la métropole), soit l'examen professionnel ouvert aux adjoints techniques ${ }^{19}$.

Dès 1954, la Tunisie compte 22 ingénieurs tunisiens diplômés et 16 sous-ingénieurs. Toutefois, dans les secteurs stratégiques comme les Mines, l'Industrie et I'Energie qui comptent 26 ingénieurs et géologues, les ingénieurs tunisiens représentent une infime minorité : un ingénieur et deux géologues. Aucun ingénieur tunisien n'exerce dans la Compagnie fermière des chemins de fer (50 ingénieurs et dirigeants subalternes ${ }^{20}$ en 1954), à la Compagnie de phosphate Sfax-Gafsa (20), et à la Compagnie d'électricité et transports. L'historienne A nousheh Karvar a évalué à 36 les diplômés des grandes écoles françaises, l'année de l'indépendance de la Tunisie $(1956)^{21}$.

Par ailleurs, les compétences techniques des mêmes ingénieurs diplômés tunisiens ne sont pas toujours utilisées d'une manière rationnelle. L'ingénieur des télécommunications Mohamed Mili a été chargé en 1947 des Transports (parc automobiles). Par obligation ou par choix personnel, la plupart d'entre eux se retrouvent au début des années 1950 dans l'A dministration, c'est-à-dire aux postes de commande politique. Ceci déplaît à quelques administrateurs coloniaux qui voient, à la veille de I'indépendance de la Tunisie, une inversion des rôles et une menace éventuelle pour les intérêts français dans le pays :

« Les ingénieurs tunisiens marquent une prédilection pour l'Administration, et une répugnance complète pour l'industrie. Sur 22 ingénieurs, 13 sont fonctionnaires, 3 en chômage ou en fuite, 6 seulement sont placés dans l'industrie ou à leur propre compte. Cette situation est d'autant plus grave pour les intérêts français que les ingénieurs tunisiens s'efforçant tous de se placer dans les services administratifs contrôlant les entreprises industrielles ou de Travaux publics françaises et à cadre français, c'est un véritable contrôle des Français par les Tunisiens qui tend à s'instaurer. » 22 
Cette situation découle-t-elle d'une véritable « stratégie d'occupation » de l'Administration par les cadres techniques tunisiens, prônée par les nationalistes tunisiens? Ou est-elle la conséquence del'exclusion des ingénieurs tunisiens des secteurs industriels ou stratégiques?

Le directeur des Travaux publics de la Tunisieconstate, de son côté: « leTunisien n'est pas portévers les carrières techniques, mais plutôt vers les carrières libérales». "Certes, écritil, il est plus difficile, pour un bachelier de mathématiques élémentaires de préparer les concours des grandes écoles que de se faire inscrire dans une faculté de droit, de médecine ou de pharmacie. D'autre part, ce n'est un secret pour personne en Tunisie que les carrières administratives spécifiquement tunisiennes sont plus lucratives que les carrières techniques. »

La sélection des élèves ingénieurs tunisiens se fait à la base. Les voies d'accès aux grandes écoles françai ses sont étroites : il existeà Tunis une seule classe de mathématiques supérieures au lycée Carnot, un lycée français. Les chances de passer le concours d'entrée par le niveau des mathématiques supérieures à l'Ecole des ingénieurs des travaux publics de l'Etat, créée au début des années 1950, sont très réduites ; ils doivent chercher d'autres filières. Cependant, il convient également de tenir compte d'une autre donnée : I'environnement culturel et économique. En effet, le modèle de réussite sociale chez les Tunisiens n'est peut-être pas encore l'ingénieur, profession accaparée par les Français, mais les professions d'avocat et de médecin beaucoup plus accessibles et échappant en grande partie au contrôle de l'Etat colonial ; sur le plan économique, le secteur industriel tunisien reste embryonnaire, malgré le développement pendant la Seconde Guerre mondiale d'une industrie « de substitution » demeurée fragile.

\section{Ingénieurs et techniciens français : l'instrument d'une politique de maintien des intérêts français en Tunisie?}

La préparation des conventions franco-tunisiennes en 1954 a montré le rôle essentiel des ingénieurs français dans le maintien des intérêts économiques et stratégiques de la France en Tunisie, sous l'apparence d'un corps réputé apolitique. Amorcée dès la fin de la Seconde Guerre mondiale, la décolonisation se concrétise progressivement en associant de plus en plus de Tunisiens à la gestion de certaines sociétés ${ }^{23}$, grâce au concours de la partie libérale et éclairée des administrateurs français. Selon le directeur des Travaux publics, dès 1948, «le raidissement du nationalisme interdisait de plus en plus au gouvernement tunisien de concéder une richesse nationale à une société capitaliste privée « étrangère » (même française). En outre, il n'existait pas de capitalistes tunisiens privés désireux d'investir dans une société par action. La solution proposée par Jean Mathieu fut donc de bâtir des compagnies d'économie mixte ${ }^{24}$ : le capital provenait de l'Etat tunisien d'une part, et des sociétés nationales et 
privées françaises et de l'Etat français d'autre part. Ces entreprises constitueront le noyau des futures entreprises nationales tunisiennes. On peut en citer quelques-unes: la Société des forces hydroélectriques de Tunisie (SFHET) constituée avec une participation d'EDF à son capital ; la Société de recherche et d'exploitation des pétroles de Tunisie (SEREPT) dont une partie du capital est fournie par la Compagnie française des pétroles ou la société de transports par avion Tunis-Air avec une participation d'Air France.

Le conseil d'administration de ces sociétés mixtes associait des fonctionnaires tunisiens à égalité avec les Français : à la SEREPT, M ohamed Ali El A nnabi, ingénieur en chef à la direction des Travaux publics, faisait partie des quatre représentants de l'Etat tunisien : Rachid Ben Osman, caïd honoraire, DeFrondeville, ingénieur en chef des Mines à la direction des Travaux publics, Boinard, sous-directeur des Impôts. Cette situation de transition constitue-t-elle une étape avant la « tunisification » complète de ces sociétés ou plutôt de leur étatisation après l'indépendance ?

Pour le directeur des Travaux publics, I'intérêt stratégique de la France exi geait un contrôle des ports maritimes de Tunis, Bizerte, et Sfax ; des transports ; et, dans certains cas, de la production et du transport de l'électricité (en interconnexion avec l'Algérie) ; enfin, un contrôle des recherches pétrolières et des exploitations minières (minerais stratégiques) ${ }^{25}$. Dans la conception de l'époque, la protection (ou le contrôle) de certains secteurs reste attachée à la notion de défense du territoire dont la France conti nue à avoir la charge. Pour la même raison, la direction des Travaux publics propose le maintien en Tunisie d'ingénieurs français, notamment dans les secteurs des transports d'une part, et d'autre part, des mines ${ }^{26}$, carburants et électricité.

L'enjeu est d'importance à la veille de l'indépendance tunisienne: par le biais des ingénieurs, la France possède, entreautres, deux atouts majeurs : la technicité, et les plans relatifs à la défense du territoire. D'autant plus que les ingénieurs tunisiens ne sont pas légion dans ces secteurs : dans les services routiers, on compte un seul ingénieur (principal) tunisien sur 39 ingénieurs français de différents grades ; au service topographique, sur les 77 ingénieurs, 14 sont tunisiens, dont un ingénieur principal, chef de service ; le service maritime ne compte aucun ingénieur tunisien parmi les 10 ingénieurs opérant dans ce service ${ }^{27}$. II arrive que des ingénieurs tunisiens occupent la place de premier plan dans certains services (les plus anciens ?), comme celui des bâtiments civils ou le service topographique. 


\section{INGÉNIEURS ET VIE ASSOCIATIVE}

Si la participation des quelques ingénieurs tunisiens aux services techniques est difficile dans le système colonial qui les marginalise, en revanche, leur participation à la vie sociale et culturelle est intense. Quelques figures émergent : nous citerons deux exemples: celui de Mohamed Ali El Annabi, premier ingénieur polytechnicien tunisien (promotion 1929) qui a été pendant plusieurs années le président de l'Association des anciens élèves du collège Sadiki. II donnait souvent des conférences, encourageait les étudiants à poursuivre des études en France à travers I'A ssociation des étudiants nordafricains en France. Le second exemple est celui de Mohamed Mili, ingénieur des communications, qui a été pendant les années 1950 président de l'Association tunisienne des caravanes des jeunes (1951). Parmi les Français, un grand nombre d'ingénieurs ont animé diverses associations : d'assistance mutuelle, associations régionales, culturelles ${ }^{28}$ ou de bienfaisance mais aussi à caractère technique ou scientifique. Ces groupements amicalistes ou professionnels sont autant d'indicateurs de la constitution de corps professionnels ${ }^{29}$ conscients de leur identité professionnelle, souvent confondue avec l'identiténationale.

\section{Association amicale du personnel des travaux publics (15 juin 1905)}

Constituée le 15 juin 1905 par arrêté du Premier ministre, la Société amicale et d'assistance mutuelle du personnel des travaux publics a l'originalité de défendre le personnel français local contre le personnel métropolitain. Elle demande que le recrutement se fasse sur un critère de compétence parmi la catégorie d'employés qu'elle représente. Elle se propose «d'apporter son appui pour la sauvegarde des intérêts moraux et matériels de ses membres » 30 et traite en priorité des questions de statuts, de traitements, concernant le personnel des Travaux publics. En janvier 1906, la Société compte 226 membres actifs ${ }^{31}$ appartenant à tous les services, 126 membres adhérents et 3 membres associés. Ces derniers, classés parmi les membres bienfaiteurs, sont les ingénieurs Léon Boulle, Jomier, Vincent et Alphonse Durel, outre Eugène de Fages, directeur général des Travaux publics. Cette association française est ouverte à toutes les catégories de personnel des Travaux publics.

\section{U nion des ingénieurs et techniciens français - groupe de Tunisie (janvier 1945)}

L'Union des ingénieurs et techniciens français serait I'héritière de l'Association des ingénieurs et techniciens tunisiens. Dès 1937, date de sa création, celle-ci a demandé à être reconnue par l'administration, mais nous avons peu de renseignements sur ce groupement. 
$\mathrm{Au}$ lendemain de la Seconde Guerre mondiale, des groupements réunissant ingénieurs et techniciens vont se constituer en France et dans les colonies. En 1945 est créée I'Union des ingénieurs et techniciens en France et dans les colonies (UNITEC) qui se veut « un vaste rassemblement des ingénieurs et techniciens »32; elle agit en liaison avec le mouvement de la Résistance française, insiste dans son bulletin sur le rôle social de I'ingénieur ${ }^{33}$. Un groupement semblable est créé à Alger (1944) ${ }^{34}$ et un autre en Tunisie I'UNITEC-groupe de Tunisie (janvier 1945). L'UNITEC-Tunisie publie un bulletin qui contient, outre des renseignements sur la vie du groupe, des études d'ingénieurs sur des thèmes d'actualité : le développement de l'électricité en Tunisie, l'agriculture, l'urbanisme. Cette association regroupe 384 membres dont 240 sont à Tunis : ingénieurs, techniciens et contremaîtres. Lors de son assemblée générale du 12 mai 1945, elle émet le regret que « les camarades tunisiens sont encore trop peu nombreux $\gg$ parmi eux ${ }^{35}$.

\section{La Fédération des associations des ingénieurs de Tunisie (1950-1956) : les ingénieurs « sans les techniciens »}

Créée en 1948 par le centralien Jean Tommy-M artin, comme un lieu de rassemblement de tous les ingénieurs, la Fédération des associations des ingénieurs de Tunisie (FAIT) est reconnue deux ans plus tard par arrêté du 13 octobre 1950. Elle a pour but :

« a) d'étudier toutes les questions d'ordre technique, social et moral intéressant I'ensemble des ingénieurs et de mettre en œuvre des mesures propres à apporter des solutions à ces questions ;

b) d'établir des liaisons amicales permanentes entre les ingénieurs et entre leurs corps (et) leurs groupements régionaux.»

Les membres actifs de droit sont les anciens élèves d'une école représentée en Tunisie par une association dont ils sont membres. Cette organisation française groupe également des ingénieurs tunisiens sortis des écoles métropolitaines. Parmi ces derniers, A bdelaziz Zenaïdi a occupé, pendant quelques mois, la fonction de trésorier au sein du bureau exécutif de la Fédération (1952). Le montant de la cotisation annuelle est assez élevé : 100 francs pour les membres actifs, inscrits à une association d'école et 200 francs pour les autres ${ }^{36}$.

En 1952, la FAIT regroupe environ 800 ingénieurs provenant de quatorze écoles différentes et répartis dans diverses professions et localités du pays ; un peu plus de la moitié (402) sont des ingénieurs agricoles (ou agronomes) que nous évoquons ici à titre comparatif ${ }^{37}$. Cependant, nombreux sont les gadz'A rts sortis des A rts et Métiers (103 tous 
Français), suivis des ingénieurs de Polytechnique (75 dont un Tunisien). Elle a été présidée successivement (1948-1951) par Jean Tommy-Martin, ingénieur de Centrale, Germain A badie, polytechnicien (1909), Antonin Coupin, ingénieur de l'Institut national agronomique, Georges Michel, ingénieur de l'Ecole centrale des arts et manufactures (1920) et Joseph Descotes-Genon, ingénieur IEG (1920). Outre la solidarité «traditionnelle » qui unit les ingénieurs d'une même école, le Bulletin de la Fédération des associations d'ingénieurs de Tunisie (paru de novembre 1950 à 1956), va rechercher à rapprocher les ingénieurs des différentes écoles. Solidarité de corps et solidarité française doivent se confondre. Selon le président de la FAIT, la mission de la Fédération ne doit pas s'arrêter à encourager la camaraderie « localisée pour le moment entre les ingénieurs d'une même école», elle doit déborder « sur un plan plus général, sur le plan ingénieur... surtout dans ce pays où nous représentons tous, à quelque titre que ce soit, la technique française ${ }^{38}$. A cette fin, la FAIT a publié à deux reprises les listes nominatives des ingénieurs par école. Ces données nous permettent d'apprécier à grands traits la physionomie du corps des ingénieurs en Tunisie au début des années 1950 (cf. le tableau en annexe).

Les principales activités de la Fédération des associations des ingénieurs de Tunisie sont l'organisation des conférences, des visites d'usines ou de barrages (en 1955, usine SOTAL, sondage de la SEREPT à Protville, les silos de Ben Arous, etc.) ; et, parfois, de sites archéologiques. Les thèmes traités par le bulletin concernent l'extraction du pétrole; I'organisation technique du pays, son équipement et la technique industrielle en général ; ainsi que le béton. Elle fait appel parfois à des ingénieurs français de passage à Tunis, comme Lebegue, ingénieur l'Ecole des arts et manufactures, membre de l'Association française des conseils en organisation qui a prononcé en mai 1950, une conférence sur «L'organisation du travail. Méthode des temps élémentaires », à la salle de l'Alliance française à Tunis. Des visites d'entreprises sont organi sées périodiquement. Un «Bal des ingénieurs » a lieu tous les ans au Majestic Hôtel. La FAIT a souhaité la construction de la Maison de l'ingénieur à l'instar de certaines villes métropolitaines.

A la lecture de ses rapports d'activité, la Fédération, soucieuse avant tout de la solidarité du corps des ingénieurs, passe sous silence les tensions ou conflits qui surgissent entre ingénieurs français et ingénieurs tunisiens et de ces derniers avec I'administration du Protectorat. La compétence de certains ingénieurs tunisiens est mise en cause ${ }^{39}$. De plus, un grand nombre d'entre eux ne sont pas affectés à des tâches techniques mais occupent des emplois administratifs. 
Nous ignorons encore les raisons qui ont empêché certains ingénieurs tunisiens d'adhérer à la Fédération des associations d'ingénieurs de Tunisie; des raisons politiques peuvent être avancées : à partir de 1952, et probablement à la suite des opérations de la résistance armée, $A$. Zénaïdi, trésorier de la Fédération, n'assiste plus aux réunions des assemblées générales de cette organisation ; Tahar Amira, ingénieur des Mines (promotion 1945), était engagé très tôt dans l'action nationaliste et plus tard en soutenant la résistance armée ; Ezzeddine Abbassi, ingénieur des TP (1946), inspecteur, futur ministre des Travaux publics dans le ministère Tahar Ben A mmar (1955), est « très mal vu par le personnel français ».

\section{Q UELQUES FIGURES D'INGÉNIEURS : DES ITINÉRAIRES CONTRADICTOIRES}

L'établissement de notices biographiques de certaines figures marquantes du corps d'ingénieurs de Tunisie devrait nous aider à mieux comprendre certains faits sociaux, économiques et culturels de la Tunisie coloniale. Nous posons quelques jalons en attendant de les compléter ultérieurement. Nous présenterons ici quelques figures d'ingénieurs issus des PTT et des Travaux publics. Nous avons tenté de retracer l'itinéraire de chaque ingénieur, mais les informations recueillies jusquelà sont inégales et insuffisantes pour évoquer le parcours individuel ainsi que l'insertion sociale et politique de chaque cas étudié. L'établissement de ces biographies est en cours d'achèvement.

\section{Les ingénieurs des PTT}

BEN CHEIKH Habib. Né à Mahdia en 1928. Etudes primaires à Mahdia et études secondaires au collège Sadiki à Tunis. II poursuit des études à l'Ecole supérieure des télécommunications à Paris et obtient le titre d'ingénieur des Télécommunications. De retour à Tunis en 1952, il est recruté au titre d'ingénieur au ministère des PTT. Nommé directeur général de la Radio et de la Télévision tunisienne (1968-72), puis ministre des PTT. Source : L'Ingénieur tunisien , mars-avril 2001, p. 26.

CA BAN TOUS Jean, A riel. Ingénieur délégué des PTT, est nommé le $1^{\text {er }}$ décembre 1942 chef de service à la direction de l'Office tunisien des PTT. Source : A rchives nationales de Tunisie, carton $n^{\circ} 305,1 / 6, f^{0} 20$.

CLEMENT. Sous-ingénieur en 1937 à l'Office postal tunisien, faisant fonction d'inspecteur dans les services électriques. Source : A nnuairetunisien, Tunis, 1937. 
D EZES J ean Louis. Né en 1902 à Montbrisson (Loire). Fils d'un magistrat qui a débuté en Oranie et terminé sa carrière comme conseiller à la cour d'appel de Toulouse. A près des études au lycée de Toulouse, il est admis à l'Ecole polytechnique en 1921. II en sort dans le cadre des ingénieurs des Postes et des Télégraphes. A près un passage dans diverses écoles d'application, il est nomméingénieur à Lille, puis à Montpellier et enfin à A lger en 1931. En Algérie, où il est promu chef des PTT en 1938, cet ingénieur en chef est spécialement chargé de l'instal lation de la téléphonie automatique à Oran, Constantine, Bône, et Alger. Il prend la direction des services régionaux en Algérie. En 1941, il est appelé par l'amiral Estéva à diriger l'Office tunisien des postes et des télégraphes (19411953).

LE GOURRIEREC René. Ingénieur des Postes, Télégraphes et Téléphone ; chef de service à l'Office tunisien des PTT du $1^{\text {ex }}$ avril 1948 jusqu'à 1952. Source: A rchives nationales de Tunisie, carton $n^{\circ} 305,1 / 6$, fo 66 .

LE SA G ET Louis. Ingénieur de première classe des Télécommunications. Nommé le $1^{\text {e }}$ août 1952 chef de service à l'Office des postes et des télécommunications de Tunisie.

K HOUADJA I brahim. Néà Mahdia en 1927. L'un des premiers élèves du collège Sadiki à avoir passé le baccal auréat mathématiques (1947), après les réformes de l'enseignement dispensé dans ce collège. II poursuit des études supérieures en télécommunications et obtient le titre d'ingénieur des Télécommunications (1954). Il a travaillé en France pendant un an. De retour à Tunis, il est recruté en tant qu'ingénieur adjoint. II est directeur puis directeur général des Télécommunications (1966) ; secrétaire d'Etat aux PTT (1979), ministre du Transport et des Télécommunications puis ministre des Télécommunications jusqu'à sa retraite en 1989. Source : L'Ingénieur tunisien, mars-avril 2001, p. 25.

MILI M ohamed, Hasard et persévérance résument l'itinéraire de Mohamed El Mili. Né le 4 décembre 1917 à Djemmal (Sahel de Sousse), d'un père notaire ( $\mathrm{dl}$ ). II obtient en 1930 le certificat d'études primaires à l'école franco-arabe. Son père, qui a voulu faire de lui un instituteur, l'inscrit au collège de Sousse où il obtient en 1934 le brevet élémentaire. II réussit le concours d'entrée à l'Ecole normale de Tunis où il obtient le brevet supérieur. Admis au concours de l'Ecole normale de Toulouse en 4 année (1938-1939), après avoir passé un premier examen de « vérification des connaissances » en mathématiques et en physique, une sorte de sélection préalable. Admis en 1939, à l'Ecole normale supérieure 
de Saint-Cloud. Pendant la guerre, il enseigne pendant deux ans au collège technique Emile Loubet à Tunis. En même temps, il prépare une licence de mathématiques par correspondance avec Alger. II retourne en 1941, en pleine guerre, à Paris à I'Ecole nationale supérieure de Saint-Cloud pour passer différents certificats. Titulaire en 1944 d'une licence de la Sorbonne, il obtient la même année le diplôme de professeur. A cette époque, l'Ecole nationale supérieure des télécommunications était encore peu connue et les filières qui y conduisent aussi. Mili un «matheux » qui voulait être ingénieur, est informé «par hasard » : le «doyen » Khereddine Haqqi, étudiant syrien d'Alep, l'a renseigné sur les conditions d'entrée (avoir cinq certificats de licence). Elève de l'Ecole nationale des télécommunications de 1944 à 1946, il en sort ingénieur civil des Télécommunications et présente sa candidature pour être recruté en tant qu'ingénieur à l'Office postal de Tunisie ; sa candidature est refusée par « manque de postes disponibles ». En réalité, on refuse l'entrée d'un destourien dans un secteur qui relève de la Sécurité de l'Etat. En 1948, le département des Télécommunications, espérant rencontrer un refus du candidat, propose à Mohamed Mili un poste d'ingénieur adjoint (équivalent à celui d'un technicien français) et le charge du parc des voitures, jusqu'à 1952. Soutenu par le syndicat tunisien de I'UGTT qui, en accord avec les partis nationalistes, pousse les Tunisiens à entrer dans la fonction publique, Mili accepte un poste inférieur à son gradeà l'Office postal. II est le premier tunisien à entrer à la direction del'Office postal ; il porte le fez, dit-il, « pour se distinguer ». En 1952, à cause de pannes fréquentes, il est accusé par le directeur de l'Office de sabotage de voitures (Mili venait en fait de recruter des chauffeurs tunisiens). Vu l'importance politique de son poste, et « ne cherchant pas l'éclat », Mili se défend de toute action de sabotage. En revanche, étudiant à Paris, il a servi pendant la Seconde Guerre mondiale, après la fuite de Habib Thameur, d'agent de liaison pour les militants destouriens entre Tunis et le Caire (diffusion des lettres). Ingénieur en chef des PTT depuis 1957, il est nommé en 1975 directeur général des télécommunications et représentera la Tunisie à I'Union internationale des télécommunications dont il est actuellement secrétaire général honoraire. II a enseigné, dès 1948, les mathématiques en arabe à la Khaldounia ; il a présidé l'association scoute L'Espoir et l'A ssociation tunisienne des caravanes de jeunes (1951). Source : Entretien avec M. Mohamed Mili le 7 octobre 2000, qu'il soit ici remercié ; L'Ingénieur tunisien, mars-avril 2001, p. 25. A rchives nationales de Tunisie, E 509, 631. 


\section{Les ingénieurs des Travaux publics}

A BASSI Ezzeddine. Né à Tunis le 19 janvier 1920, il poursuit ses études primaires et secondaires au collège Sadiki puis au lycée Carnot. Il effectue, à Alger, des études préparatoires en « Math-Spé », et passe l'oral à Paris. II part à Saint Etienne en pleine guerre et poursuit ses études à l'Ecole nationale supérieure des mines et obtient le diplôme de cette école avec le titre d'ingénieur des Travaux publics. II a été membre de la commission administrative du syndicat de I'UGTT ; mais il a été révoqué pour fait de grève, le $1^{\mathrm{e}}$ avril 1953, puis réintégréle $1^{\mathrm{er}}$ avril 1954. N ommé en septembre 1955, ministre des Travaux publics au ministère Tahar Ben Ammar. Source : Le Petit M atin, Tunis, 18 septembre 1955 ; L'Ingénieur tunisien, mars-avril 2001, p. 18.

A M IRA Tahar. Né à Tunis en 1920, originaire de Monastir. Il a poursuivi ses études secondaires au collège Sadiki jusqu'à 1941. II réussit le concours d'entrée à l'Ecole supérieure des mines de Paris et obtient le titre d'ingénieur des Mines (1945). II poursuit encore une année de spécialisation dans les recherches minières. En 1946, il est recruté à la direction des Travaux publics. II a travaillé dans plusieurs sociétés : à la SEREPT. (recherche pétrolière), entre 1947 et 1956 ; à la SOFOMECA (1965-1972) ; à la SITEP (19721981). II a présidé l'A ssociation des ingénieurs et techniciens tunisiens. II a adhéré très jeune au parti du Néo-Destour (dès les années 1930) et s'est engagé dans l'action nationaliste en apportant son concours à la lutte armée ; opposant politique dans les rangs du mouvement youssefiste (1955-1956), il est emprisonné par le Gouvernement Bourguiba. Il a également assisté au congrès de Bandung en 1955.

BAITON. Ingénieur en chef des Ponts et Chaussées, ingénieur en chef des Services maritimes, directeur des Ports maritimes.

BEN OSMAN Lasaâd. Né le 16 février 1926. Ingénieur civil de l'Ecole nationale supérieure des mines de Paris (1949). De 1949 à 1952, il est ingénieur à la direction des Travaux publics (service de la prospection) ; il a présidé, de 1952 à 1955, le district (service) de la recherche pétrolière. A partir de 1955, il occupera plusieurs hautes fonctions dans les secteurs des Travaux publics et de l'A griculture. II a été plusieurs fois ministre. Carrière : directeur adjoint des eaux et des équipements ruraux (1955-1959) ; puis directeur dans le même domaine au ministère de l'Industrie (1959-1967) ; secrétaire d'Etat adjoint, puis secrétaire d'Etat à l'Agriculture (1967-69, 1969-70) ; ministre des Travaux publics et del'H abitat; ingénieur (principal) au ministère del'A griculture (1971- 
73) ; PDG pendant quelques mois de Tunis Air (janvier-octobre 1973) ; ministre des Transports et des Communications (1973-74), ministre de l'Equipement et de l'H abitat (1974-79), ministre de l'Agriculture (1979-88). Ayant également occupé plusieurs postes honorifiques à l'étranger, il préside, depuis 1993, I'Union tunisienne de solidarité sociale. Source : L'Ingénieur tunisien, mars-avril 2001, (N.S.), p. 24.

BEN SA LAH Salah. Premier ingénieur tunisien des Mines (1920), né en 1888 à Moknine. II fait des études secondaires au collège Sadiki (1901-1902), puis au lycée Carnot de Tunis où il obtient le baccalauréat (section mathématiques). En 1911, il s'inscrit à l'Ecole des mines de Paris et obtient le diplôme d'ingénieur des Mines. Les renseignements sur S. Ben Salah sont vagues : nous savons qu'il a été recruté en France, pendant la Première Guerre mondiale, par une compagnie française dont nous ignorons le nom. II retourne en Tunisie à la fin de la guerre, mais il ne peut être embauché par la direction des Travaux publics. Il s'oriente vers le commerce (société de boissons, puis vente de matériaux de construction à Sousse). Rappelé par la même compagnie française qui l'avait embauché en France, il est employé au Congo. II meurt à Brazzaville dans des conditions non élucidées. Source : ACHOUR Abdelwahab, «Salah Ben Salah », Revue de Sadiki, Tunis, $n^{\circ}$ 17, janvier 2000 (en arabe).

BON N EN FANT Jean-Louis. Ingénieur des Ponts et Chaussées à Soissons (Aine) ; en détachement à Tunis du 10 octobre 1932 au 16 février 1945, date à laquelle il est réintégré en métropole. Proposé pour la direction des TP en Tunisie (le 6 mars 1945) en remplacement de Gosselin.

BOUSOFFARA Hassen. Ingénieur (I-1947 LS). Professeur au collège Emile Loubet à Tunis, membre du Groupe de Tunisie des ingénieurs IEG.-EIH.et IRG. en 1952 [en cours d'étude]. Source: L'Ingénieur tunisien, $n^{\circ} 25$, mars-avril 2001 (N .S.), p. 14 ; Bulletin dela Fédération des associations d'ingénieurs de Tunisie, n², juillet 1952, p. 38.

DE FA GES DE LA TOUR Eugène. Ingénieur en chef des Ponts et Chaussées de $2^{\mathrm{e}}$ classe, directeur général des Travaux publics en Tunisie (1904-1908 ?). Source : Archives du Quai d'O rsay, nouvelle série Tunisie, carton $n^{\circ} 579$, fo 15 .

DE FRONDEVILLE. Ingénieur en chef des Mines, directeur des carburants en Tunisie vers 1954. 
D ELLA G I M ustapha. Ingénieur (1946) [en cours d'étude].

EL AN N A BI M ohamed Ali. Né le 15 février 1906 à Bizerte, il a fait ses études au collège Sadiki puis au lycée Carnot. Diplômé del'Ecolesupérieure de langue et littératurearabes, il suit la classe de mathématiques spéciales au lycée Saint-Louis à Paris. Premier tunisien reçu à Centrale et à Polytechnique, il intègre l'Ecole nationale supérieure des mines. Licencié es-lettres à la Sorbonne et diplômé de l'Ecole libre des sciences politiques, il a refusé une proposition d'enseigner les sciences à la Sorbonne pour ne pas avoir à renoncer à la nationalité tunisienne. M.A. El Annabi a participé activement à la vie associative en Tunisie. II est I'un des fondateurs en 1928 de l'A ssociation des étudiants musulmans nord-africains en France (A EMNAF). Il est élu président de l'A ssociation des anciens élèves du collège Sadiki (AAECS) de 1934 à 1954. Suiteaux difficultés rencontrées par l'A EMN AF pendant l'après-guerre, il utilise l'AAECS pour organiser la Quinzaine de I'étudiant, manifestation visant à recueillir des fonds d'aide aux étudiants tunisiens en France. II a fondé I'A ssociation des ingénieurs et techniciens (1937 ou 1948 ?) ; membre du Groupement de Tunisie des ingénieurs de I'Ecole polytechnique (1950-56). II est nommé en 1934 ingénieur adjoint au chef du service des Mines à la direction des Travaux publics. Muté en 1937 au secrétariat général du gouvernement tunisien pour s'occuper des problèmes du paysannat indigène et de l'artisanat. Ingénieur en chef à la direction des Travaux publics (service topographique). En 1956, il est nomméingénieur en chef des TP, et directeur du Plan. En qualité de responsable du service de Recherche scientifique et de l'Energie atomique en Tunisie ; a été membre-conseiller de la délégation tunisienne qui a participé à la session del'A gence internationale pour l'énergie atomique (AIEA - 20 septembre-20 octobre 1956). En défendant les avantages liés à l'utilisation pacifique de l'énergie atomique, notamment dans le domaine de l'hydraulique et de l'agriculture, il a convaincu Habib Bourguiba de faire adhérer la Tunisie à cette Agence. II a lui-même effectué des recherches dans les domaines de la physique de la terre, de la physiologie végétale, de l'énergie, etc. II meurt en avril 1962. Source : ARROUAS, Livre d'or, Zédition, Tunis, 1942, p. 11 ; L'ingénieur tunisien, n 25, mars-avril 2001 (N .S.), p. 13 : biographie de M.A. El A nnabi à l'occasion de sa décoration à titre posthume; EL ANNABI M.A., «L'énergie atomique au service du Centre et du Sud », L'A ction, Tunis, 10 septembre 1956.

EL FEKIH A bdelhamid. Ingénieur (1950) [en cours d'étude]. 
FA BRE A uguste. Ingénieur en chef à la Compagnie du gaz et Régie co-intéressée des eaux à Tunis [en cours d'étude]. Source : A rchives du Q uai d'O rsay., Tunisie 1944-49, carton n ${ }^{\circ}$, $f^{0} 248$.

GHEDAMSI M ohamed. Ingénieur (I-1946), membre du Groupe de Tunisie des ingénieurs IEG.-EIH .et IRG. en 1952, attaché à la Compagnie fermière des chemins de fer tunisiens, Tunis-Grombalia [en cours d'étude]. Source : L'Ingénieur tunisien, $n^{\circ} 25$, mars-avril 2001 (N.S.), p. 14. ; Bulletin de la Fédération des associations d'ingénieurs de Tunisie, n², juillet 1952, p. 38.

G IRO U D. Ingénieur en chef des Transports, directeur des Transports (vers 1954).

G OSSELIN . Ingénieur en chef des Travaux publics de la Tunisie et directeur des TP ; détaché en Tunisie le premier juin 1922 jusqu'au $1^{\mathrm{R}}$ mars 1945 par période quinquennale, demande son détachement au ministère français de la Reconstruction et de I'Urbanisme le $1^{\text {G }}$ mars 1945.

H O UKI A hmed. Ingénieur des Arts et Métiers (signalé en 1954) [en cours d'étude].

LATIRI M okhtar. Né à Hammam-Sousse, le 18 mars 1925, cet ingénieur polytechnicien est une «figure-clé » des ingénieurs tunisiens. A près des études primaires à l'école franco-arabe de sa ville natale, « ses brillants résultats » 40 lui permettent d'obtenir une bourse au collège de Sousse où il est interne. Avec l'aide de son frère, étudiant à I'université de la Zitouna, il entre au lycée Carnot et sera reçu premier au baccalauréat français. II surmonte des difficultés matérielles en rassemblant des dons privés pour payer ses frais du voyage à Paris. II s'inscrit au lycée Louis-Le-Grand puis à l'Ecole polytechnique d'où il sort diplômé en 1947. Mais en 1951, l'administration coloniale refuse de l'engager au poste d'adjoint à l'ingénieur en chef des Ponts et Chaussées. Ingénieur principal à la direction des TP, puis au ministère des TP, chef de I'arrondissement de Sousse (1956). De 1958 à 1966, il est à la tête de la direction générale de l'Equipement au ministère des Travaux publics et de l'Habitat. Nommé PDG de la société Les Industries chimiques maghrébines (1966-68). Membre du Groupement de Tunisie des ingénieurs de l'Ecole polytechnique (1952). 
MATHIEU Jean. Directeur général des Travaux publics, président d'honneur de la Fédération des associations d'ingénieurs de Tunisie (1952-1955).

MICHAUD Paul-A imé-Clément. Ingénieur en chef des Ponts et Chaussées, ancien inspecteur général. Né le 15 mars 1844 à Péron (Ain), il entre en 1864 à l'Ecole polytechnique et en sort en 1866 dans le corps des Ponts et Chaussées. II débute comme ingénieur ordinaire dans le département du Jura chargé du service ordinaire et du service hydraulique (1869-1871) ; puis dans les départements de l'Ain et du Rhône (1871-1874), chargé du service des études et des travaux des chemins de fer à Saint-Juste (1875-1883). Promu ingénieur en chef en 1883. Massicault, ancien préfet du Rhône, nommé résident général àTunis, l'appelleà lesuivreen « terre africaine » et lui confiele poste de directeur général des Travaux publics : il gère alors plusieurs services encore rudimentaires mais ses responsabilités sont assez étendues ; il dirige à la fois les Ponts et Chaussées, la police des ports de commerce et de la navigation, les mines, le service topographique, et celui des forêts. A près la mort du résident Massicault, il accepte en Corse un poste inférieur à son grade.

MICHAUX Lucien. Ingénieur des Ponts et Chaussées, adjoint au directeur général des Travaux publics (1906). Source : Revue tunisienne, $15^{\circ}$ année, $n^{\circ} 67$, janvier 1908.

MONTMARIN Arnaud de. Ingénieur principal des Travaux publics, responsable du mouvement Vie nouvelle, membre du Comité d'action franco-tunisien pour l'amitié et la coopération, créé le 5 avril 1955 à Tunis.

PETREMENT Charles-Marcel. Ingénieur, directeur de la Société nord-africaine d'électricité, gaz et eaux à Sfax. Source : A rchives du Q uai d'O rsay., Tunisie 1944-49, carton n 15 , $f^{0} 248$.

PORCHE G eorges. Ingénieur des travaux de la ville de Tunis (1906). Source : Revue tunisienne, $n^{\circ} 67$, janvier 1908, p. 92.

ZEN A ID I A bdelaziz. Né à Tunis en 1914, il a obtenu le baccalauréat au lycée Carnot de Tunis. Entre 1935 et 1937, élève du lycée Saint-Louis à Paris, il réussit le concours d'entrée à l'Ecole centrale de Paris et en sort en 1940. II reprend des études supérieures en 1941- 
1942 mal gréles difficultés liées à la guerre et devient ingénieur de l'Ecole centrale des arts et manufactures (1942). Employé pendant deux ans aux usines Renault en France, il revient à Tunis fin 1944. Il est alors employé au Commissariat à la reconstruction à Tunis et devient ingénieur en chef en 1947. II est responsable de la reconstruction, de l'habitat et de l'aménagement du territoire jusqu'à 1969, date à laquelle il est nommé PDG de la société AI Boniane (société de bâtiment qui comprend 22 filiales en Tunisie). De 1973 à 1978, il est le PDG. deTunis Air. Membre du Groupe de Tunisie des ingénieurs de l'Ecole centrale des arts et manufactures, il est le trésorier, en 1952, de la Fédération des associations d'ingénieurs de Tunisie. Mais n'assistant plus aux réunions de cette fédération, il est remplacé par Justin Blanc. Source : Biographie de A bdelaziz Zénaïdi parueà I'occasion de sa décoration, in L'Ingénieur tunisien, n² 25, mars-avril 2001 (N.S.), p. 14. ; Bull letin de la Fédération des associations d'ingénieurs de Tunisie, n² 2, juillet 1952, p. 36.

\section{N OTES}

${ }^{1}$ Sur l'évolution des figures de l'ingénieur colonial, voir VACHER Hélène, « Les figures del'ingénieur colonial à la fin du XIXe siède : la formation de la Société française des ingénieurs coloniaux et de l'Ecole Spéciale des Travaux Publics », Le M ouvement Social, n 189, octobre-décembre 1999, pp. 47-65.

2 Voir à ce propos la thèse de KARVAR Anousheh, La formation des élites scientifiques et techniques étrangères à I'Ecole polytechnique auX XIX e et XX $X^{\circledR}$ siècles, thèse d'épistémologie, d'histoire des sciences et des techniques, Université Paris VII-Denis Diderot, 1997.

3 Direction générale des Travaux publics, Les Travaux publics du Protectorat français en Tunisie, Tunis, imprimerie générale J. Picard et Cie, 1900, t. 1, p. 7. Entre 1883 et 1889, le plus grand nombre des agents du service des Ponts et Chaussées appartient «soit au corps des Ponts et Chaussées soit à l'ancien personnel du cadre auxiliaire des travaux de l'Etat», in Direction générale des Travaux publics, Compte rendu de la marche des services de 1883 à 1889, Tunis, La Rapide, 1889, p. 7.

4 BEN SALEM Lilia, «La profession d'ingénieur en Tunisie, approche historique», in Bâtisseurs et bureaucrates. Ingénieurs et société au M aghreb et au M oyen-O rient, Maison del'Orient, Lyon, CNRS, 1990; «Les ingénieurs tunisiens au XIX et XXe siècles », Revue de la M éditerranée et du M onde musulman, 72, 1994/2, pp. 60-74.

5 KHELFAOUI Hocine, Les ingénieurs dans le système éducatif. L'aventure des instituts technologiques algériens, Paris, Publisud, 2000, p. 20.

6 L'Office postal est créé par une convention signée le 29 mars 1888 entre le ministre des Affaires étrangères, le ministre des Finances, pour les Postes et les Télégraphes et le résident général, comme représentant du bey. L'Office postal entre en activitéle ${ }^{\star}$ juillet 1888; il est justiciable de la Justice française: « L'objet de ses services, qui assurent l'échange des correspondances de l'Etat en même temps que cel les de particuliers, et aussi le caractère politique, administratif, militaire même de ses opérations, réclament ces

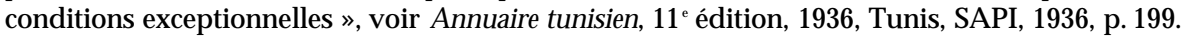

7 Un premier repérage dans les archives du ministère français des Affaires étrangères nous a permis de recenser un peu plus d'une vingtaine de cartons sur les Travaux publics en Tunisie et sur la Poste.

8 Voir à ce propos PLANEL A nne-Marie, D ela N ation à la colonie : la communautéfrançaise deTunisieau $\mathrm{XIX}$ e siècle, d'après les archives civiles et notariées du consulat général de France à Tunis, thèse d'histoire, Paris, EHESS, 2000, $773 \mathrm{p}$. 
9 Paul Michaud, ingénieur en chef des Ponts et Chaussées, directeur général des Travaux publics sous le résident général Massicault, a accepté, à la mort de ce dernier, un poste inférieur à son grade en Corse. Bonneau, ingénieur des Ponts et Chaussées à la direction des Travaux publics de Tunisie, a demandé à être mis à la disposition du gouvernement ottoman, mais il n'a pu être libéré, «il remplit à la direction des T.P. des fonctions importantes, comprenant notamment la construction d'une ligne ferrée de première urgence », Le résident général S. Pichon au ministre des Affaires étrangères, le 2 octobre 1908, A rchives du ministère des A ffaires étrangères (MAE), nouvelle série Tunisie, carton $n^{\circ} 579$.

10 BELAID Habib, « Les PTT dans la Régence deTunis : enjeux politiques et militaires auX XIXe et XXe siècles», Rawafid nI, 1995, (revue de l'Institut supérieur d'histoire du mouvement national), Tunis, pp. 168149; «Les PTT dans la Régence de Tunis : enjeux politiques et mi litaires aux XIX`et XXe siècles », R awafid n I, 1995, (revue de l'Institut supérieur d'histoire du mouvement national), Tunis, pp. 168-149; « Les débuts de la radiodiffusion en Tunisie (1935-1946) », Revue tunisienne de communication, revue de l'Institut de presse et des sciences de l'information (IPSI ), Tunis, 1997.

11 Extrait deLa D épêche tunisienne du $1^{\text {e }}$ avril 1891. voir aussi Bulletin de l'O ffice des postes et télégraphe,

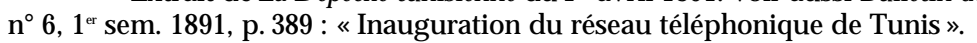

12 Ibid.

13 |bid.

14 Créé pour assurer l'application de la loi foncière de 1885, le service topographique est chargé de procéder aux travaux de reconnaissance, de bornage, de triangulation, d'arpentage et de lotissement et l'établissement des plans cadastraux. En 1946, il compte 18 ingénieurs topographes (tous français) qui dirigent une centaine de topographes ou aspirants topographes (agents commissionnés), en plus des agents techniques, Note sur la création du diplôme de géomètreexpert, en date du 2 avril 1946, le résident général Mast au ministère des Affaires étrangère, Archives du M AE, série Tunisie 1944-50, carton n 161, p. 153-154.

15 Cetteconcession remonteà une convention de 1872 , renouveléeen 1884 . Notice sur la direction des Travaux publics, avec une liste nominative du personnel, A nnuaire tunisien, Tunis, SAPI, 1936.

16 Construction de barrages : 7 étaient prévus à la fin des années 40, 3 étaient en cours en 1948 (Sidi Salem, Oued Ellil, Oued Mellègue).

17 On a recruté, dans l'immédiat après-guerre, des ingénieurs suisses (2 ou 3), mais on n'a jamais évoqué la formation d'ingénieurs tunisiens.

18 «Rémunération des fonctionnaires de la direction des Travaux publics de Tunisie », rapport de mission de M. Vincent, inspecteur général des Ponts et chaussées, Paris, le 12 décembre 1949, A rchives du M AE, sérieTunisie 1944-50, carton $n^{\circ} 162$, f $^{\circ} 168$.

19 «Rémunération des fonctionnaires ...», rapport de mission de M. Vincent, inspecteur général des ponts et chaussées, Paris, le 12 décembre 1949, A rchives du M AE, série Tunisie 1944-50, carton n 162, f 168.

20 En 1954, la Compagnie fermière des chemins de fer compte 4000 agents d'exécution, dont 2100 français, 1950 tunisiens et 150 algériens ; au chemin de fer de Gafsa, le nombre d'agents d'exécution est de 860 sur un effectif total de 2000 agents ; sur le réseau de la Compagnie tunisienne d'électricité et des transports, l'effectif du personnel est de 950 tunisiens pour seulement 320 français. Voir ministère du Commerce, Intérêts français, Industries diverses, le 26 juillet 1954, A rchives du M AE, série Tunisie 1950-55, carton $n^{\circ} 397, f^{\circ} 88$.

${ }^{21}$ Ces ingénieurs sont répartis comme suit : 4 diplômés de l'Ecole polytechnique, 10 de l'Ecole des mines de Paris et de Saint-Etienne, 4 de l'Ecole d'hydraulique de Grenoble, 3 de l'Ecole des télécommunications, 2 de l'Ecole des travaux publics et 1 del'Ecole centrale des arts et manufactures de Paris, Voir KARVAR A., La formation des élites scientifiques et techniques... , op. cit., p. 266.

22 Ministère de l'Industrie à Tunis, «note sur service des industries diverses », Intérêts français, A rchives du M AE, sérieT unisie 1950-55, carton $n^{\circ} 397, f^{\circ} 96$.

23 « J'ai toujours prôné l'association franche et complète avec les Tunisiens sur le plan économique, écrit Jean Mathieu, ingénieur en chef, directeur des Travaux publics. $\mathrm{N}$ 'ayant pas réussi et je le déplore à convaincre beaucoup des industriels français en Tunisie, j'ai essayéau moins dans mon domaine, c'est-à-dire dans le secteur public ou para-étatique, de créer des associations », in «Etude pour servir à l'élaboration des conventions franco-tunisiennes » en date du 11 août 1954, Archives du M AE , série Tunisie 1950-1951, carton $n^{\circ} 397$, bobine $693, f^{\circ} 9$. 
24 MATHIEU Jean, «Etude pour servir à l'élaboration des conventions franco-tunisiennes » en date du 11 août 1954, A rchives du M AE, série Tunisie 1950-1951, carton $n^{\circ} 397$, bobine 693, f० 9.

25 MATHIEU Jean, «Etude pour servir... »?, A rchives du M AE, série Tunisie 1950-1951, carton n³97, bobine $693, f^{\circ} 13$.

26 Le syndicat de I'UGTT et le parti du Néo-Destour revendiquent depuis des années la nationalisation des mines.

27 A rchives du M AE, sérieTunisie 1950-1951, carton n 397.

${ }^{28}$ En 1908, 13ingénieurs français et un géomètretunisien attachéàl'administration des Habous (Chadly EL Okby, 1902) sont abonnés à la Revuetunisienne, cf. Revue tunisienne, $15^{\mathrm{e}}$ année, $n^{\circ} 67$, janvier 1908, p. 92.

29 Voir à ce propos : LUCAS Yvette, DUBAR Claude (Eds), Genèse et dynamique des groupes professionnels, Presses Universitaires de Lille, 1994.

30 Bulletin dela Sociéé amicale et d'assistance mutuelle du personnel des travaux publics, n 1, février 1906, p. 8.

31 Dont 14 agents métropolitains. Voir Bulletin cité, n 1, février 1906, p. 11

32 Bulletin de l'U N ITEC, Tunis, $n^{\circ}$ 6, (mensuel), juin 1945.

33 Dans un article, BOUCHARD, secrétaire général de I'UNITEC, «L'UNITEC au service de la France » trace le programme de cette association : « Nous, ingénieurs et techniciens, qui avons pu acquérir des connaissances générales et techniques, avons le devoir national et social d'en faire bénéficier nos subordonnés (...) Nous avons un rôle éducateur à remplir auprès du personnel sous nos ordres (...) Créons des centres d'orientation et de formation professionnelles. Faisons de l'apprentissage une réalité et non une caricature », Bulletin de I'U NITEC, $n^{\circ} 6$, juin 1945, pp. 14-15.

${ }^{34}$ CegroupeU N ITEC-A Igérieou U nion des Ingénieurs et techniciens français (puis d'A I gérie) est reconnu d'utilité publique par décret du 22 octobre 1947 ; il publie un bulletin trimestriel (puis bimestriel à partir de mai 1958), le Bulletin d'information, de documentation et d'études. En janvier 1956, ce groupement compte 364 adhérents et 328 «amis de I'UNITEC », présidé par MABILLOT puis RICHARD, d'après son bulletin n³3, nouvelle série (10A Anné), février 1956.

35 Bulletin de l'U NITEC, Tunis, $n^{\circ}$ 5, mai 1945, p. 9.

36 Article 5 des Statuts de la FAIT, Bulletin de la FA IT, n 1, novembre 1950, p. 5.

37 L'Ecole coloniale d'agriculture de Tunis a été créée en 1898.

38 DESCOTES-GEN ON, président de la FAIT, voir Bulletin de la FAIT, n² 2, juillet 1952.

39 «II n'est pas douteux que la tournure d'esprit des Tunisiens les porte aux arguties juridiques et aux discussions byzantines dans lesquelles les intérêts personnels jouent toujours un rôle primordial. A notre connaissance, seuls deux ingénieurs de l'Administration font vraiment preuve du souci de l'intérêt public », Ministère del'Industrieà Tunis, Service des industries diverses, Intérêts de la France, 28 juillet 1954, A rchives du MAE, série Tunisie 1950-1951, carton n³97, bobine 693, f 96.

${ }^{40}$ Sur la biographie de M. LATIRI, voir KA RVAR A., La formation des élites scientifiques... , op. cit., p. 274. 


\section{Annexe}

Etat des groupements affiliés à la Fédération des associations d'ingénieurs de Tunisie* (en juillet 1952 ) :

\begin{tabular}{|c|c|c|}
\hline Associations & $\begin{array}{l}\text { Nombre total } \\
\text { d'adhérents }\end{array}$ & $\begin{array}{c}\text { Dont } \\
\text { Tunisiens }\end{array}$ \\
\hline Groupe de Tunisie des ingénieurs des arts et métiers & 103 & 0 \\
\hline $\begin{array}{l}\text { Groupe de Tunisie des ingénieurs de l'Ecole de } \\
\text { l'électricité }\end{array}$ & 23 & 0 \\
\hline $\begin{array}{l}\text { Groupement de Tunisie des ingénieurs del'Ecole centrale } \\
\text { des arts et manufactures }\end{array}$ & $20^{* *}$ & 1 \\
\hline $\begin{array}{l}\text { Groupement de Tunisie des ingénieurs I.E.G.-E.I.H. et } \\
\text { I.R.G. }\end{array}$ & 50 & 2 \\
\hline $\begin{array}{l}\text { Groupement de Tunisie des ingénieurs del'Ecole } \\
\text { d'ingénieurs deMarseille }\end{array}$ & 23 & 6 \\
\hline $\begin{array}{l}\text { Groupement de Tunisie des ingénieurs des Instituts } \\
\text { catholiques des arts et métiers }\end{array}$ & 9 & 0 \\
\hline $\begin{array}{l}\text { Groupement de Tunisie des ingénieurs del'Ecolede } \\
\text { physique et de chimie industrielle }\end{array}$ & 3 & 0 \\
\hline $\begin{array}{l}\text { Groupement de Tunisiedes ingénieurs de l'Ecole } \\
\text { polytechnique }\end{array}$ & 75 & 1 \\
\hline $\begin{array}{l}\text { Groupement de Tunisie des ingénieurs des Ecoles de } \\
\text { travaux publics }\end{array}$ & 49 & 0 \\
\hline $\begin{array}{l}\text { Groupement de Tunisie des ingénieurs del'Institut } \\
\text { électrotechnique deToulouse }\end{array}$ & 5 & 0 \\
\hline $\begin{array}{l}\text { Groupement deTunis des ingénieurs agricolos ; } \\
\text { Groupement deTunisie des ingénieurs agricolos; } \\
\text { Groupement de Tunis des ingénieurs agricoles** }\end{array}$ & 357 & 22 \\
\hline Groupement de Tunisie des ingénieurs agronomes & 49 & 0 \\
\hline Ingénieurs autodidactes de Tunisie & 3 & 0 \\
\hline Total & 769 & 32 \\
\hline Pourcentage & 96 & 4 \\
\hline
\end{tabular}

Source : Bulletin de la Fédération des associations des ingénieurs de Tunisie, n² 2, juillet 1952

*Comptage fait à partir des listes nominatives des ingénieurs publiées par le Bulletin dela Fédération des associations des ingénieurs de Tunisie, n² 2, juillet 1952.

**Dont une femme française (promotion de 1922).

*** Ces trois groupements figurant sur trois listes différentes du bulletin de la FA IT ont été réunis au sein d'une même rubrique : Groupement de Tunis des ingénieurs agricolos (186 adhérents dont 13 Tunisiens), Groupement de Tunisie des ingénieurs agricolos (120 dont 9 Tunisiens), et Groupement de Tunisie des ingénieurs agricoles (51 tous Français). 



\title{
Du métier à la profession : I'émergence de I'ingénieur géomètre et l'exercice colonial au début du $X X^{e}$ siècle
}

\author{
Hélène VACHER
}

L'article propose d'examiner le développement de la profession d'ingénieur géomètre au début du $X X^{e}$ siècle, en questionnant les effets de l'intégration de nouveaux territoires dans l'«Empire colonial » sur ce processus. Notre analyse porte sur les pratiques professionnelles et techniques qui se sont forgées dans l'espace colonial, à la fois à distance du creuset métropolitain et en dynamique avec la «modernisation » du métier de géomètre en France. A la fin du XIX siècle, les géomètres sont surtout associés au monde rural, où ils travaillent pour leur très grande majorité, et ils se présentent rarement comme des ingénieurs. Certes, il arrive parfois qu'ils exercent à ce titre lorsqu'ils sont passés par une grande école, ou bien après avoir suivi des cours de topographie au Conservatoire national des arts et métiers ou de géodésie au Service géographique de l'armée. Ce n'est qu'après la Première Guerre mondiale qu'émergent des ingénieurs géomètres, munis d'un diplôme de l'Ecole spéciale des travaux publics (ESTP) reconnu par l'Etat en $1922^{1}$; ils développent une culture technique qui participe d'une nouvelle identité professionnelle.

En cernant les parcours et les activités de géomètres ou de topographes qui ont été des agents techniques de la transformation des territoires coloniaux, on observe un phénomène de décentrage professionnel, relativement au cadre de référence métropolitain, qui semble un vecteur de « rénovation » des professions elles-mêmes. La «mise en valeur des territoires neufs $»^{2}$ mobilise d'abord des techniques exportées par la métropole ; elleen suscite aussi, ou du moins transforme le cadre de référence de l'action technique d'agents investis dans un espace échappant en partie au cadre de référence métropolitain de leur exercice. 


\section{LE TERRITOIRE COLONIAL : RECONNAISSANCE, SÉCURITÉ, TRANSFORMATION}

Au début du $X X^{e}$ siècle, le profil du géomètre topographe se dessine à travers plusieurs associations qui envisagent la création d'un syndicat professionnel à l'échelle nationale et une revalorisation du métier de géomètre à partir d'une formation technique reconnue. C'est durant cette même période que s'affermit en France une politique de gestion et de mise en valeur des territoires coloniaux :

«A une époque où la vapeur et l'électricité ont, en quelque sorte, rétréci les bornes du monde et suscité entre les peuples une concurrence plus âpre, il n'est pas de col onisation possible sans voies de communication. $»^{3}$

Le lancement d'importants programmes d'équipement dans plusieurs régions de l'Empire colonial naissant mobilise les compétences en topographie et en géodésie pour la mise en cartes des territoires. Les géomètres sont aussi mis à contribution avec leurs techniques et connaissances nécessaires à la restructuration économique et juridique de ces territoires. Entre les domaines de la géodésie et de la topographie (placés sous la responsabilité des services de l'armée) et ceux de la topométrie urbaine ou rurale, l'éventail des formations et des statuts professionnels est largement ouvert.

L'organisation de sociétés professionnelles accompagne le renforcement de I'encadrement technique attaché à la politique de «mise en valeur » des territoires conquis. A insi, la Société française des ingénieurs coloniaux (SFIC), fondée en 1895, avait pour premier objectif d'offrir un «point de ralliement » pour les ingénieurs établis ou mutés aux colonies. Par ailleurs, elle voulait constituer une tribune (publications, conférences, etc.) pour diffuser les « procédés de travail » et les « goûts spéciaux » de ces techniciens. Ces derniers étaient amenés à développer des formules innovantes en adaptant leurs opérations techniques à des conditions particul ières, tout en recherchant le moindre coût pour leurs réalisations. La SFIC rassemblait à la fois des ingénieurs civils, souvent membres de la Société des ingénieurs civils de France (SICF), et des ingénieurs des Ponts et Chaussées. Elle reçut le patronage d'ingénieurs éminents tel qu'Emile Cheysson (1839-1910), inspecteur général des Ponts et Chaussées, connu pour ses travaux de statistiques et pour ses conceptions de la « science sociale ${ }^{4}$. Groupant près de 250 membres, un an après sa création, alors que la SICF en rassemblait un nombre dix fois supérieur, la SFIC s'est rapidement consolidée en doublant ses effectifs au tournant du siècle. Elle constituait un centre d'échange d'informations professionnelles et para-professionnelles pour les techniciens métropolitains et coloniaux ${ }^{5}$. Son centre de gravité était porté par la branche du génie civil, fer de lance de la « mise en valeur des territoires neufs », et plus particulièrement par les secteurs liés 
au déploiement des infrastructures portuaires ou urbaines et au fonctionnement de leurs réseaux. La Société avait partie liée avec un puissant milieu entrepreneurial souvent associé au « parti colonial », les interactions entre ces divers groupements restant encore à préciser 6 .

Cette orientation de la SFIC vers les activités du bâtiment et des travaux publics n'était cependant pas exclusive. Des géographes ou des topographes s'exprimaient fréquemment dans son bulletin et la Société de topographie de France (STF), créée en 1876, fai sait partie des organisations affiliées ${ }^{7}$. H. N audin, chef des travaux géodésiques et topographiques de l'Ecole centrale des arts et manufactures, était ainsi membre du comité central de la SFIC avant la Première Guerre mondiale. Dès les premières années de sa publication, le bulletin de la SFIC rend compte des innovations dans les techniques de levé topographique utilisant la photographie ou celles concernant les instruments susceptibles de faciliter les opérations $d u$ «topographe colonial $»^{8}$. A près la Première guerre mondiale, le bulletin expose les nouvelles techniques d'enregistrement et de réorganisation du foncier.

Le rôle des sociétés de géographie dans la propagation de «l'idée coloniale » et I'influence de la géographie coloniale dans le développement de la discipline ont fait l'objet d'études substantielles 9 . On sait aussi que, lors des périodes de déclin des activités cartographiques en France auX XVIII et $\mathrm{XIX}^{\mathrm{e}}$ siècles, le champ colonial a permis aux « techniciens de la géographie », plus particulièrement aux personnels militaires spécialisés, de se lancer dans des missions scientifiques ${ }^{10}$. Cependant, les activités des ingénieurs topographes et a fortiori celles des géomètres forment un référent moins courant.

Au congrès international des géomètres de Bruxelles en 1910, un conférencier, chargé du cours de topographie à I'université de Liège, présentait «la carte » comme un instrument essentiel du renforcement des empires coloniaux. « La carte» fournit en effet des données susceptibles d'informer à des fins de mise en valeur, elle permet aussi d'assurer la sécurité du territoire :

«La mise en valeur d'une colonie et sa bonne administration exigent que l'on connaisse la configuration de son sol, son étendue et ses limites, ses voies de communication et son régime hydrométrique, ses richesses minérales, la distribution des races qui la composent, leur puissance relative, leurs ressources et les limites de leurs territoires. D elà, la nécessité absolue de posséder des cartes qui nous renseignent avec le plus d'exactitude sur tous ces éléments dont beaucoup peuvent d'ailleurs servir également à la répression de troubles occasionnels à l'intérieur, aussi bien qu'à la défense éventuelle du territoire. »11 
Un quart de siècle plus tard, en 1931, à l'occasion du congrès colonial international de Paris, Jean Lecq insistait sur la complémentarité des champs de compétences :

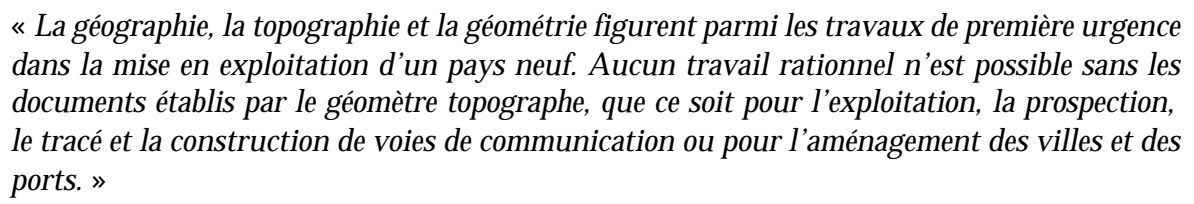

Ce diplômé de l'Ecole supérieure de topographie (1928), faisait observer que, à l'ère « des défricheurs », «les premières conquêtes coloniales ont été accompagnées de documents topographiques $\gg 12$.

Entre le soldat et l'ingénieur constructeur, l'ingénieur topographe travaille au contrôle des territoires conquis, à leur insertion dans des dispositifs techniques, juridiques et fonciers exportés par la colonisation, et à leur intégration dans un réseau territorial polarisé par la métropole ${ }^{13}$. II précède souvent l'ingénieur constructeur ou l'ingénieur agricole comme agent de transformation et de mise en valeur. Les services topographiques ont d'ailleurs été créés à la suite des différentes vagues de colonisation : 1848 pour le service topographique de l'Algérie ; 1868 pour celui de la Cochinchine; 1882 pour le Protectorat tunisien ; 1896 et 1898 pour ceux de Madagascar et de N ouvelleCalédonie ; 1910 pour I'AOF créée en 1895, 1926 pour l'AEF créée en 1908, etc. Les géomètres œuvrent également à la préparation de l'implantation des réseaux en complétant les travaux du génie militaire ou des conducteurs des services des travaux publics. Ils opèrent surtout dans le domaine de la réforme de l'assiette fiscale en créant les parcellaires qui constituent la plateforme territoriale de la colonisation. Ces professions, dont les techniques se recouvrent en partie, travaillent de concert à la reconnaissance et à la mise en forme des territoires coloniaux.

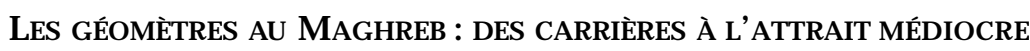

En 1891, le D ictionnaire des professions et métiers fait ressortir le caractère libre de la profession de géomètre en France, l'absence d'enseignement officiel et la concurrence qui pèse sur ce métier. Celui-ci jouit d'un médiocre reconnaissance dans la mesure où il peut être exercé par «quiconque possède les connaissances mathématiques, juridiques et techniques nécessaires ». En revanche, ce même guide professionnel remarque qu'un « débouché important et assez avantageux est ouvert aux géomètres en Algérie et en Tunisie, pays nouveaux où la nécessité de constituer la propriété $a$ entraîné la création de services topographiques complets $\gg^{14}$. Rappelons que l'organisation de ces services, leur 
composition, la quantité de leurs personnels et leurs attributions varient fortement suivant les périodes et les régions de l'Empire. De même, la condition des géomètres aux colonies diffère sel on les conditions del'implantation coloniale et les régimes fonciers préexistants.

La Société nationale des géomètres de France, d'Algérie et de Tunisie (SNGFAT) constituée en 1893, après la scission de la Société des géomètres français (SGF) de 1847 (dite «Comité central »), reste peu disserte sur l'affiliation de ses groupements al gériens et tunisiens. La faible représentation des géomètres et topographes coloniaux, évidente Iors du congrès international de Bruxelles en 1910 et du congrès national de Paris en 1913, témoigne de la difficile structuration de l'organisation corporative dans un cadre colonial. Selon la SNGFAT, le statut professionnel et les conditions d'exercice des géomètres au Service topographique tunisien ne semblent pas répondre aux promesses énoncées par le Dictionnaire. Les perspectives de carrière y sont en effet peu enviables avec des traitements de 2400 francs par an ${ }^{15}$. De plus, c'est «une vie de lutte, de sacrifice et de travail » qui attend les géomètres expatriés. N'ayant pas de caisse de retraite comme les employés des services de travaux publics, «presque tous (les géomètres) meurent en fonction ${ }^{16}$. Le correspondant du J ournal des géomètres experts fait pourtant l'éloge de ces employés qu'il présente comme «la pierre d'édifice de l'Immatriculation », car «si I'officier a planté les jal ons de l'occupation en temps de guerre, le géomètre est venu les consolider en temps de paix $\gg 17$. Le tableau que dresse le géomètre-expert $E$. Pinçon, pour l'Algérie ne témoigne pas plus du prestige de la profession. Le service topographique qu'il décrit emploie 116 géomètres, 6 vérificateurs et 3 géomètres en chef qui procèdent aux travaux d'usage, triangulations, levés, nivellements :

«Tant dans l'intérêt des services de la Colonisation, des domaines et des forêts que pour les opérations concernant l'application du senatus-consulte de 1863 : délimitations et levés des douars, circonscriptions administratives en pays musulman ; et (dans l'intérêt) de la loi du 16 février 1897 sur la constatation de la propriété privée et la constitution de la propriété indigène par le système des enquêtes partielles. »18

L'organisation professionnelle demeurait «embryonnaire » dans la mesure où ses membres, tous fonctionnaires, étaient préoccupés « presque exclusivement de revendications pécuniaires ${ }^{19}$. Quant aux géomètres non contractuels, beaucoup plus nombreux que les fonctionnaires, ils sont dépourvus d'associations et n'ont, pour la plupart, qu'un faible niveau de qualification. Pinçon exprime en 1912 les difficultés qu'il y aurait pour le géomètre français à s'acclimater à «la mentalité cosmopolite » et de «pénétrer l'élément autochtone »; et il appelle ses collègues à «provoquer une organisation » pour aider à l'installation des géomètres arrivant de métropole. Toutefois, 
il semble que I'Union des géomètres experts français (UGEF), formée en 1920, n'a pas hérité d'un noyau d'organisation corporative outre-mer, ce que revendiquait la SN GFAT, malgré son intérêt affiché pour le développement de l'exercice colonial 20.

A la lecture des documents émanant des organisations professionnelles du début du XXe siècle, la structuration du milieu des géomètres, dans le cadre colonial, semble donc très en retrait par rapport à celle des fonctionnaires des travaux publics. II en ressort, en particulier, une forte disparité des traitements entre les agents des travaux publics et ceux des services topographiques, à niveau égal de compétence. La situation des géomètres «coloniaux » serait reléguée dans les rangs inférieurs des personnels techniques qui choisissent des carrières outremer et cela, quelles que soient les formes d'exercice, au sein des services topographiques ou pour des cabinets privés. Les horizons d'attente s'ouvrent cependant en 1912 après I'instauration d'un protectorat au Maroc.

\section{Le Protectorat marocain et le renouvellement professionnel}

Le Protectorat français au Maroc impulse une nouvelle dynamique à la profession grâce à un «redéploiement spatial » s'appuyant d'une part sur une capitalisation des pratiques coloniales d'implantations antérieures et, d'autre part, sur des techniques et des disciplines nouvelles. Nous retiendrons trois spécialisations qui structurent le profil de «l'ingénieur géomètre ». La topographie en fait « un agent de reconnaissance », la topométrie, le droit et l'économie en font un «agent de rationalisation de l'assiette fiscale » ou « un agent technique de l'aménagement des villes ».

\section{La topographie de reconnaissance}

Le rôle d'avant-garde dans la conquête joué par ceux qui effectuent les «premiers coups de sonde », est connu, ainsi que leurs motivations scientifiques plus ou moins teintées d'exotisme ${ }^{21}$. Parmi les nombreuses figures d'explorateurs, celle du marquis de Segonzac est emblématique. II parcourt le Rif au début du siècle, déguisé « sous les traits d'un mendiant misérable», avant d'être chargé par le Comité du Maroc d'une mission de reconnaissance dans le $\mathrm{N}$ ord et le Sud marocain en 1904-1905. II devait par la suite présider la Société de géographie du $\mathrm{Maroc}^{22}$. Cette page « héroïque » de l'histoire du « vieux Maroc », selon la terminologie coloniale du moment, est tournée avec l'occupation de Casablanca et la création en 1908 du « Bureau topographique des troupes débarquées», futur Bureau topographique du Maroc (BTM). Le Service géographique de 
l'A rmée (SGA) entend maintenir dans toutes les colonies des noyaux d'officiers rompus aux reconnaissances de terrain, sans pour autant créer une structure permanente et spécialisée, comme celle de l'ancien corps des ingénieurs géographes. Aussi proposet-il d'utiliser au Maroc les groupes d'ingénieurs qui ont été formés dans les brigades géodésiques et topographiques d'Algérie et de Tunisie : ils y auraient acquis « beaucoup de coup d'œil, beaucoup d 'habileté, l'usage de la topographie, une grande pratique de la

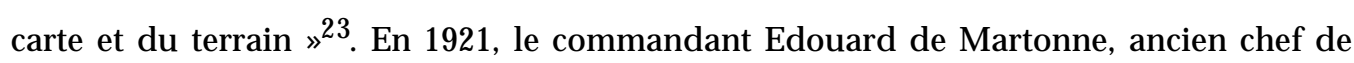
brigade topographique à M adagascar, confirme cette tactique :

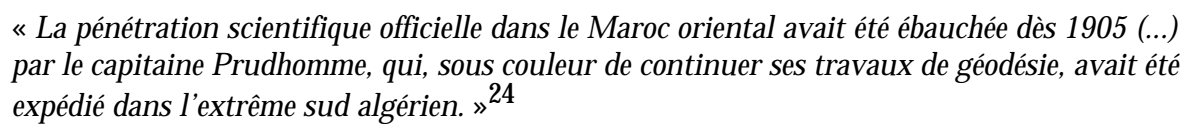

C'est d'abord avec l'appui d'un bureau topographiqueà Oran, à partir de décembre 1907, que des travaux sont entrepris pour établir une carte au 1/ 200000 « des confins algéro-marocains ». IIs se poursuivent grâce à l'ouverture d'un bureau topographique à Oujda. Pour le SGA, qui supervise tous ces travaux, il s'agit de développer les méthodes d'une géodésie et d'une topographie de campagne, tout en répondant aux besoins des troupes en information cartographique, ainsi qu'aux demandes des services civils qui se développent au fur et à mesure de la « pacification ». Edouard de Martonne nommé, dans l'après-guerre, à la direction de la section topographique du Service géographique du Maroc (SGM) 25 affirmeà ce propos :

«La topographie n'est qu'un auxiliaire de la conquête et de la pacification, elle marche exactement avec l'une et suit de près les progrès de l'autre, elle doit couvrir d'un levé parfois approximatif des étendues considérables de terrain, se pliant aux nécessités militaires, ne pouvant se permettre de laisser sans une représentation même approchée tel coin de terrain à peine entr'aperçu, mais où elle ne reviendra peut-être pas d'ici longtemps, et où le coup de sonde quelle a jeté peut servir plus tard à faciliter la pénétration définitive. »26

En quoi consistel'action du SGA ? A entreprendre simultanément les opérations de géodésie et de topographie, ce qui demande des équipes expérimentées, capables de relever le terrain à mesure que progresse la pénétration militaire. Avec des moyens réduits pendant les années de guerre 1914-1918, le Bureau topographique réussit néanmoins à cartographier une partie importante du «Maroc utile», tandis que des officiers spécialisés dans les plans de ville réalisent la plupart des fonds de plans qui serviront aux urbanistes. Fonctionnant par la suite avec des moyens renforcés, le nouveau SGM est conçu comme une extension du SGA, «l'organe du commandement militaire pour les travaux de l'avant ». II poursuit la reconnaissance du territoire et se réserve l'établissement de documents qui préparent l'édition des cartes topographiques et géographiques. Un service de restitution des photographies, placé en 1917 sous la 
responsabilité du capitaine Orcel, est intégré au Bureau topographique dès mars 1919. A fin de pouvoir couvrir les zones de dissidence, les autorités du Protectorat ont introduit, peu avant la fin du conflit mondial, les toutes nouvelles techniques de la photographie aérienne qui venaient d'être testées sur le front, en Europe ${ }^{27}$. Au Maroc cependant, les difficultés techniques surpassent de beaucoup celles rencontrées en métropole, en raison del'absence de documents préal ables offrant d'indispensables repères. La dimension des territoires à relever est par ailleurs considérable :

«Il s'agissait ici, en effet, de dresser de toutes pièces, en vue d'opérations militaires, une carte de reconnaissance au $200000^{\circ}$ de la zone dissidente, dans la bande de terrain inaccessible située en avant de la ligne des postes, en représentant sur une grande profondeur le relief du sol et la planimétrie. »28

Orcel détaille «l'attrait captivant » qu'il peut y avoir à fouiller les photos de territoires inconnus, « la loupe à la main »: outre les renseignements géographiques, géologiques et économiques, « avec les photos prises au-dessus des régions dissidentes du Maroc», on arrive dit-il «à recueillir une foule de renseignements qui ne peuvent prendre place sur une carte au $200000^{\circ}$, mais qui sont d'un intérêt primordial ». La nouvelle technique permettrait de percer « en une fraction de seconde infime», le « secret des pays inconnus $»^{29}$.

La Société de géographie du Maroc, créée en mars 1916, diffuse les travaux du Bureau topographique dans les milieux professionnels concernés par les questions de topographie. Toutefois les rapports d'activités de la société regrettent le fai ble intérêt des coloniaux pour les « recherches théoriques et désintéressées »30. Placée sous le patronage des personnalités les plus en vue du Protectorat, la Société regroupe plus de 400 adhérents au sortir de la guerre; ses membres actifs comptent les officiers topographes, les conservateurs de la propriété foncière, les ingénieurs du Service des travaux publics, les chefs des services municipaux, enfin les ingénieurs géomètres et géomètres privés parmi les plus actifs dans les travaux urbains ${ }^{31}$.

\section{La réforme de l'assiette foncière}

La promulgation du dahir du 12 août 1913 sur l'immatriculation prend appui sur l'expérience des réalisations effectuées, en Tunisie (1885) et à Madagascar (1896), à partir du modèle de $I^{\prime}$ " Act Torrens » régissant le territoire australien ${ }^{32}$. Depuis la fin du XIX siècle, parallèlement aux revendications pour l'instauration en métropole d'un «livre foncier », les voix sesont multipliées pour demander une « réforme» del'assiettefoncière et un système unifié, dès «l'inclusion » de nouveaux territoires dans l'Empire ${ }^{33}$. 
En France, l'héritage du morcellement foncier de la Révolution et le partage de la gestion de la propriété immobilière entre deux professions - celle du notaire et celle du géomètre - compliquent et ralentissent à la fois la circulation des valeurs foncières. Suivant ce découplage professionnel, le cadastrage des biens fonciers revêt une signification indicative quant aux droits de propriété, les actes sur lesquels sont portées les contenances des parcelles gardant une valeur juridique essentielle en cas de litige. Ceux qui demandent une réforme du cadastre en France, à l'occasion des travaux de la commission extra-parlementaire du cadastre (1891-1905), soulignent les faiblesses juridiques et techniques du système existant dont la finalité est d'abord fiscale. Ils plaident pour que l'administration du cadastre donne à la propriété une base à la fois physique et juridique : les plans devraient être plus exacts comme référents topographiques et plus précis dans leurs indications des limites de parcelles ; en outre ces documents devraient être en concordance avec un «livre foncier » assurant la publicité des droits réels de propriété pour chaque parcelle, et ayant une valeur juridique. Un cadastre multi-fonctionnel, en quelque sorte, permettant une plus grande souplesse des transactions foncières. Mais, si les partisans d'une « réforme cadastrale » globale ont pu compter sur d'importants appuis institutionnels, on pense ici à la Société de topographie parcellaire animée par Joseph Louis Sanguet, leur projet n'aura pas abouti en France ${ }^{34}$.

En revanche, pour l'élite technophile, qui est alors généralement favorable à la politique coloniale de l'association, cette réforme paraît adéquate outre-mer, dans la mesure où elle favorise le passage entre « anciens » et «nouveaux » régimes fonciers. Dans l'esprit des « techniciens de la colonisation », l'occupation d'un territoire sous-entend un changement de forme, une amélioration volontariste et, surtout, le passage d'un ordre ancien, perçu comme obscur, archaïque et régi par la stratification de droits d'usage traditionnels, à un système moderne, cohérent, basé sur l'expertise rationnelle. Dans cette optique, les territoires reformés doivent servir de support à l'activité entrepreneuriale. L'introduction d'une nouvelle assise foncière est censée faire bénéficier tout un chacun de cette rationalisation du foncier, les indigènes pouvant en théorie accéder au nouveau régime au fur et à mesure de leurs besoins. A insi, le système mis en place en Tunisie avec la loi du $1^{\star}$ juillet 1885, modifiée par les lois et décret des 15 et 16 mars 1892, et celui mis en place au Maroc serviront de références pour le milieu professionnel des géomètres, tant métropolitains que coloniaux, durant l'entre-deux-guerres. A vec des ajustements, ce même modèle avait été repris, en 1896, par Gallieni pour avoir fait « ses preuves en Australie, en Nouvelles Galles du Sud et en Tunisie » ; il avait essaiméégalement, au Congo (1899) et en 
A OF (1906) $)^{35}$. Ce système visait à assurer les nouveaux droits de propriété, à rendre les transactions plus sûres et à favoriser le développement d'un marché hypothécaire. Dans les années 1930, les géomètres le montrent en modèle, car ils y voient une manière d'assurer « à la propriété immobilière une sécurité parfaite et aux transactions des facilités telles que les propriétaires, en Tunisie, semblent avoir, à tous égards, une situation plus avantageuse que celles réglementées par les législations d'Europe »36.

En Tunisie, en effet, c'est l'administration qui, par le biais du service de Conservation de la propriété foncière, établit le titre de propriété à partir des éléments fournis par un tribunal mixte, composé, pour moitié, de magistrats français et de magistrats tunisiens. Par ailleurs, la forme et les limites matérielles de la propriété sont fixées par le service topographique, chargé de dresser les plans des propriétés soumises à immatriculation et de les tenir à jour. Les personnels recrutés et formés par ce service, à partir de 1886, se retrouvent fréquemment à Madagascar, au Maroc, ou encore dans les «Régions libérées », en France, au lendemain de la Première Guerre mondiale. En principe, la publicité de la procédure et l'examen par le tribunal des prétentions contradictoires doivent faire échec à toute tentative de spoliation. Les professionnels contemporains sont fascinés par le fait que la propriété acquiert une sorte d' «état civil », une individualité juridique, quasi autonome face au propriétaire: «Ce dernier peut se comparer à un actionnaire : le titre est susceptible d'être négocié ou engagé, par conséquent l'immeuble est, pour ainsi dire, mobilisé »37.

Au Maroc, le dahir du 12 août 1913 introduit progressivement un système d'immatriculation constituant des titres inaliénables qui, selon le commentaire du code de 1925, « purge et détruit tous les droits réels antérieurs à l'inscription d'un immeuble déterminée sur un livre foncier qui fait foi, à l'exclusion de tout ce qui n'y est pas inscrit »38. L'administration du Protectorat crée par arrêté « viziriel » le 26 octobre 1915 un corps d'agents topographes des Domaines et de la Conservation de la propriété foncière ${ }^{39}$. Une alternative est donc offerte au régime immobilier musulman qui est invariablement présenté comme «des plus complexes »40. A la différence du système tunisien qui prévoit l'arbitrage d'un tribunal, le système marocain est exclusivement administratif, la décision d'immatriculation se faisant sous la responsabilité d'un conservateur.

Les dispositions du dahir - dont celles ouvrant sur l'immatriculation automatique en cas de non-opposition - ont soulevé de vives critiques chez les experts qui redoutaient une aggravation des risques de spoliation 41 . Pourtant, on remarque que les controverses 
sur l'organisation juridique du système foncier en terres coloniales ne sont guère débattues par la presse corporative qui met en avant la nouveauté du système administratif du Protectorat, susceptible d'accélérer l'entrée des terres indigènes dans l'économie générale et de stimuler le crédit foncier, ce préalable à « la mobilisation des sols ». On fait aussi largement état des travaux du service de la Conservation foncière, exécutés pendant les années du conflit mondial, sous la direction de Marc Roussel, en soulignant la qualité technique des documents produits ${ }^{42}$. L'exposition coloniale de Marseille en 1922 offre l'occasion de présenter ces réalisations, notamment des atlas, des travaux d'immatriculation et les plans de lotissements de Casablanca dressés par les géomètres Bardin, Buan, Berthet et Eal et ${ }^{43}$. Lors del'exposition col oniale de Vincennes en 1931, le même service topographique peut montrer plusieurs exécutions de remaniement parcellaire, dont le plan d'ensemble des levés d'immatriculation des villes de Casablanca et de Fedhala sur la côte de la Chaouia44. L'organisation et la gestion cadastrales des territoires de l'«Empire colonial » constituent un pôle d'innovations techniques pour la métropole, tout en favorisant les opportunités de mise en cohérence des stratégies de modernisation administrative et juridique. Lorsque la rénovation générale du cadastre français fait à nouveau l'objet de discussions passionnées dans un milieu professionnel qui déplore la portée restreinte de la loi du 16 avril 1930, le Maroc est souvent pris en exemple, sous la forme d'une image lissée des avantages qu'offrirait un système de cadastre « rationnel ».

\section{Filière coloniale et pratiQues URbanistiQues : LeS ingénieurs géomètres DE RABAT À DAMAS}

Dans la réforme du territoire au Maroc sont juxtaposés des modes de gestion nouveaux du foncier et des systèmes hérités, régis par la «tradition », qui ne disparaissent pas totalement. Parallèlement, un dispositif global d'aménagement instaure un régime d'expropriation et de remembrement original qui fait référence pour I'urbanisme français entre les deux guerres ${ }^{45}$. II s'exporte aussi dans les territoires placés sous mandat français. C'est à partir du « chantier marocain » qu'un réseau de géomètrestopographes a pu se constituer du Maroc jusqu'en Syrie.

Dès 1907, les conditions de travail des géomètres au Maroc avaient retenu I'attention de la SNGFAT. En effet, son président, Rémi Frère, avait étélégèrement agressé par «les soldats de Raisouli » lors d'une opération de bornage. Le Journal des géomètres experts rapporta d'autres incidents, déplorant la précarité de la situation dans l'Empire 
chérifien ${ }^{46}$. En 1909, les projets de cadastre puis, en 1913, la préparation des plans d'extension suscitent un vif intérêt ${ }^{47}$. L'ESTP où s'est créé à partir de 1905 un enseignement de topographie ${ }^{48}$ parvient en 1915-1916 à mettre sur pied une section marocaine deson amicaled'anciens élèves: celle-ci regroupe aussi bien des ingénieurs des travaux publics que des géomètres topographes. A vant la Première Guerre mondiale, le service de placement de l'amicale de I'ESTP indiquait déjà que le Maroc offrait un débouché pour les géomètres recherchant un emploi, en exercice libéral ou dans l'administration. On demandait surtout des conducteurs de travaux publics, mais aussi des opérateurs et des chefs de brigades tachéomètriques, de même que des ingénieurs topographes ou des géomètres. Ainsi parut en 1913 une annonce pour une place dans le cabinet du géomètre $A$. Tardif, chargé du cadastre de Casablanca et auteur des premiers projets du plan d'aménagement de la ville ${ }^{49}$. Pendant la guerre, les propositions faisant appel à des aides géomètres et à des géomètres augmentent. Les annonces insistent désormais sur les qualifications professionnelles et techniques : la connaissance de la méthode des coordonnées rectangulaires et des logarithmes, la capacité à relever le plan entier d'un quartier, la maîtrise d'utilisation du tachéomètre, etc. Si diplômes ou certificats sont parfois demandés sans plus de précision, il arrive que les compétences requises soient spécifiées ; par exemple : «Les ingénieurs géomètres doivent connaître la géodésie (et être) capables de faire de la triangulation et des levés parcellaires ». Les traitements proposés s'élèvent à 3000 francs par an, avec des indemnités de 1000 francs $^{50}$.

A la fin du conflit mondial, les autorités du Protectorat décident de créer un enseignement pour les géomètres ${ }^{51}$. Un premier établissement existait depuis 1918 à Casablanca, sous lenom de «Compagnie des géomètres ». Un arrêté « viziriel » du 25 juin 1920 le rattache à la section civile du SGM en le transférant à Rabat. II prend alors le nom $d^{\prime}$ « Ecole de géomètres et de dessinateurs ${ }^{52}$. Recrutant des élèves français et marocains, le nouvel établissement est divisé en trois sections : élèves géomètres (niveau baccalauréat) ; élèves dessinateurs français (brevet simple) ; élèves indigènes (avec certificat d'études secondaires) ${ }^{53}$. II a pour mission de former le personnel du nouveau SGM. Cette tentative de développer un enseignement technique s'inscrit dans un moment de transition institutionnelle : le Protectorat veut alors transférer aux services civils les compétences de la section militaire du service géographique. Le Journal des géomètres experts mentionne que l'Ecole de Rabat ouvre ses portes sous la direction du centralien M. Michel, chef des services topographiques, avec vingt-deux élèves inscrits pour neuf mois de cours et de stages pratiques ; il rapporte aussi que «le directeur de 
l'Ecole constate l'insuffisance du temps qui lui est accordé pour la formation de ses géomètres $\$^{54}$. Dès 1922, cet établissement est fermé. Deux raisons sont invoquées : le manque d'élèves ayant un niveau d'instruction suffisant et l'offre réduite d'embauche par l'administration du Protectorat. Des cours de perfectionnement sont néanmoins conservés pour permettre la préparation des agents aux examens de l'administration 55 . Par ailleurs, Léon Eyrolles, directeur de I'ESTP, annonce l'ouverture au sein de son établissement parisien d'une nouvelle école pour le $1^{\sigma}$ janvier 1922 : «Sur la demande du ministère des Régions libérées et du résident général du Maroc, nous venons d'ajouter (...) une quatrième école, l'Ecole des géomètres et topographes, qui deviendra l'année prochaine l'Ecole supérieure de topographie „56. Aussi, les programmes de cet enseignement spécialisé sont-ils établis avec la participation du service de la Conservation foncière du Maroc. La renaissance de l'Ecole supérieure de topographie, créée avant 1914 mais pratiquement éteinte pendant les années de conflit, coïncide bien avec la quasi-extinction de I'Ecole de Rabat. Trois bourses de 3000 francs sont même attribuées par les autorités du Protectorat en 1922, les lauréats devant être employés au service de la Conservation foncière ${ }^{57}$. Mais, on est bien loin des promesses de débouchés, pour des « centaines » de géomètres, évoquées par René Danger en 191958 .

La croissance rapide de Casablanca génère la constitution d'un groupe actif de géomètres urbains. Les annuaires mettent au jour une dizaine de noms de géomètres, parmi ceux qui ont participé aux projets de lotissements entrepris dans la capitale économique du Maroc ${ }^{59}$. Certains, comme S. Lapierre, ont fait un passage au Bureau topographique. Souvent, ils se présentent alternativement comme architectes et géomètres, ou commearchitectes géomètres ; c'est par exemple le cas de Wolff et de Tardif. A Rabat également, on constate la combinaison de plusieurs activités : un « cabinet topographique » désigne un cabinet d'architecte expert offrant plans topographiques et immatriculation, mais également des plans de construction pour armatures béton armé, terrasses étanches, etc. Les activités sont évolutives et les distinctions entre ingénieurs constructeurs, géomètres ou architectes semblent aléatoires. Nombre d'entre eux se retrouvent dans la Société de géographie. Ainsi le géomètre-expert S. Lapierre en est I'archiviste en 1921 et son comité comprend M. Michel, sous-directeur de la section civile du SGM, de même que $M$. Roussel de la Conservation foncière ${ }^{60}$.

A la même époque, I'organe de la SNGFAT poursuit l'orientation amorcée au congrès des géomètres de 1913. Pour positionner la profession dans le domaine de l'urbanisme, le journal cite en exemple Georges Buan qui «continue énergiquement sa 
campagne sur les plans d'aménagement et d'extension »61. Ce « géomètre assermenté de Casablanca » ne manque pas de rendre hommage au plan Prost, tout en mettant en avant les compétences spécifiques du géomètre en matière de lotissements. II esquisse un partage des rôles dans l'aménagement urbain : au géomètre revient celui de la défense des intérêts privés et de la médiation avec les municipalités.

La participation de géomètres gravitant autour de I'ESTP aux réalisations marocaines couvre un large spectre d'activités professionnelles. Le bulletin et la revue technique de I'association amicale de I'ESTP, L'Ingénieur-Constructeur, informent régulièrement sur les débouchés et sur les opérations conduites au Maroc en rendant compte des aspects techniques et juridiques de l'aménagement. Aux côtés de Bussière, chef des services municipaux, on trouve Jean Lassalle, diplômé de l'ESTP en 1919, après avoir suivi un cursus par correspondance. L'Ingénieur-Constructeur présente Lasalle, qui est vice-président de la section marocaine de l'amicale de I'ESTP installée à Rabat dans I'après-guerre, comme « ingénieur topographe ETP, vérificateur du service géographique du Maroc et ancien chef du Service topographique des plans de ville », Iorsqu'il lui ouvre ses colonnes en 1921 pour un article sur le remembrement urbain :

« L'urbanisme n'a pas uniquement en vue l'aménagement des cités futures; par des judicieuses retouches, les quartiers anciens sont mis en harmonie avec le plan d'ensemble conçu d'après les règles les plus modernes [... ] C'est par le mécanisme des redistributions que $s^{\prime}$ opèrent ces remaniements essentiels de l'assiette des fonds urbains. »

Pour l'auteur, le remaniement « rationnel » du parcellaire urbain est un prolongement naturel de la «technique du lotissement », et il donnel'exemple des mises en œuvre de remembrement et de redistribution dans un quartier de Rabat pour « souligner l'extrême complexité des opérations urbaines » et montrer l'utilité du géomètre. Cette technique de remembrement urbain, mise en place au Maroc en 1917, est fréquemment citée en exemple par bon nombre de membres de la Société française des urbanistes (SFU) : ils prônent son introduction en France pour présenter une alternative à la coûteuse procédure d'expropriation ${ }^{63}$.

Dans la seconde partie des années 1920, l'éventail de démonstration « du géomètre à la coloniale » s'élargit avec les premiers comptes rendus des opérations d'aménagement menées au Liban et en Syrie. Placé sous la direction de C. Duraffourd, le centre de Ba'abda, près de Beyrouth, est qualifié en 1929 par Le Journal des géomètres experts de première « géométropole » du monde : 150 géomètres de toutes nations y sont employés en permanence ; son encadrement est en grande partie franco-suisse, et ses moyens sont inégalés en matériels comme en hommes, avec 500 agents répartis dans les 
pays sous mandat ${ }^{64}$. L'organe de l'UGEF présente aussi le service du cadastre et de la topographie qui concentre et traite les travaux des équipes de terrain ; on se félicite de I'« éclectisme» des méthodes allant du traditionnel chaînage à la photographie aérienne en passant par la tachéométrie ${ }^{65}$ de précision. On insiste sur l'application du principe de la division du travail, avec la formation de sections spécialisées pour le lotissement et le remembrement. Dans un autre numéro du même journal, Duraffourd analyse le bouleversement des méthodes provoqué par la guerre : il détaille le développement «d'un outillage scientifique pour appliquer les méthodes nouvelles qui n'existaient pas auparavant et réduire de plus en plus les frais de main-d'œuvre, afin que le coût des levés ne soit pas disproportionné avec la valeur des terrains »66. Pour le directeur du centre de Ba'abda, l'ampleur de la tâche à accomplir dans les pays placés sous mandat aurait favorisé une organisation administrative et technique susceptible de réaliser l'unification des réformes et méthodes (collaboration internationale), la compression des personnels, la sélection des meilleurs matériels, en bref, «l'industrialisation, I'homogénéité et le meilleur rendement des travaux $\gg 7$. La profession a ici franchi un palier grâce à une remarquable concentration de moyens ; en intervenant sur l'ensemble des échelles d'opération, le géomètre s'affirme comme ingénieur.

Deux carrières sont caractéristiques de la dynamique, liée à une pratique coloniale, du groupe socioprofessionnel et de son évolution. Celle de René Danger peut ainsi se présenter selon trois dimensions. Il fut à la fois enseignant à l'Ecole supérieure de topographie et porte-parole prééminent du mouvement professionnel (au sein de la SNAGFAT et de I'UGEF), enfin directeur de l'un des plus importants cabinets de consultants en plans de villes de l'entre-deux guerres. Sa carrière est marquée par une double expérience en milieu colonial, dans le bassin méditerranéen et dans les « vieilles colonies » outre-Atlantique. Cet itinéraire a tendu à renforcer une trajectoire professionnelle centrée sur « la rationalisation » du métier. Un autre parcours est celui d'Henri Roussihle. Détaché aux colonies avant 1914 pour effectuer des levés cartographiques, cet ingénieur hydrographe devient I'un des principaux concepteurs de la photographie aérienne pendant la guerre, puis directeur du Service de la reconstitution foncière, entre 1919 et 1924. Roussilhe ne chercha pas seulement à renouveler les méthodes de la topographie et de la géodésie ; il s'attacha également à promouvoir la profession de l'ingénieur géomètre, en élaborant les programmes de l'Ecole supérieure de topographie avec René Danger et Léon Eyrolles. Leur démarche s'est inscrite dans un projet plus vaste visant à faire aboutir la «réforme cadastrale » que souhaitaient les 
milieux favorables «à une rationalisation » économique et sociale de la métropole. Pour montrer la voie dela « modernisation », Roussilhe faisait aussi bien appel aux réalisations des services fonciers du Maroc qu'à « l'organisation impeccable » impulsée par Durrafourd dans les pays sous mandat.

A la veille de l'Exposition coloniale internationale de 1931, l'A micale des géomètres du service topographique d'Alger sollicite le maréchal Lyautey pour que soient mis à l'étude les problèmes spécifiques des géomètres coloniaux. La question concerne autant leur situation professionnelle dans l'Empire, que l'adaptation des méthodes et des procédés techniques, ou encore l'analyse comparée des différents régimes fonciers et des procédures ${ }^{68}$. A la séance de clôture de l'exposition, le bilan est contrasté. Si la faiblesse du milieu corporatif colonial est un thème constamment évoqué, les uns notent le manque d'unité des formations et la dispersion des travaux exposés, alors que les autres mettent en avant l'apport considérable que représentent, pour les géomètres, l'emploi extensif de la photographie aérienne et l'accumulation d'expériences acquises par les coloniaux dans le domaine des plans de ville ${ }^{69}$. Cela vaut aussi bien pour les fonctionnaires des services topographiques de certaines colonies que pour les experts ayant fait leur spécialité du marché international des plans de ville en s'appuyant sur les réalisations engagées dans l'Empire.

\section{CONCLUSION}

A la fin du XIXe siècle, le métier de géomètre se dégage lentement de la logique cadastrale autour de laquelle gravitaient auparavant les conditions d'exercice de la profession. En déclin par rapport aux professions ascendantes portées par les dynamiques qui peuvent être rattachées à la « seconde » industrial isation, les géomètres manquent de surcroit d'une organisation forte depuis la scission intervenue en 1893. Leur identité professionnelle est menacée de deux cotés. D'une part, un grand nombre de marchés sont la proie de professions concurrentes qui disposent d'un bagage technique plus étendu, comme c'est le cas avec les conducteurs des Ponts et Chaussées. D'autre part, les rangs des géomètres privés paraissent se clairsemer, les reconversions sont fréquentes en faveur de professions plus lucratives, notamment celle d'agent immobilier. Dans ce contexte, un projet se dessine pour renouveler le métier et engager un processus de professionnalisation. Ce «sursaut » s'articule autour de l'alliance contractée par la SNGFAT et I'ESTP afin de réaliser une école de topographie ${ }^{70}$. 
A la différence d'autres professions, comme celles que regroupe le «génie civil » au sein de la SFIC, le métier de géomètre n'est pas directement confronté à «l'encombrement des carrières » durant les premières décennies du XXe siècle. Ainsi les colonies ne constituent pas à proprement parler un exutoire pour des géomètres qui ne trouveraient pas de débouchés en métropole. L'examen de la situation des géomètres aux colonies, à partir des organes de la presse professionnelle, indique une fragilité de leur statut et, plus généralement, un faible attrait du service outre-mer pour les patentés comme pour les fonctionnaires, et cela, bien que les «situations coloniales » aient fait l'objet d'une certaine promotion.

L'option coloniale se serait plutôt posée en considération de l'accès qu'elle donnait à une spécialisation professionnelle, à de nouvelles méthodes et à un nouvel outillage technique. L'Outre-mer a aussi représenté une ouverture pour une profession ancrée pour les trois quarts de ses membres dans une identité « localiste » et rurale en lui permettant de prendre pied dans de nouvelles « missions d'initiatives», en particulier celles liées à l'aménagement urbain. C'est dans cette perspective que nous avons présenté un aperçu de l'éventail des opportunités qu'offrait « un passage colonial » aux géomètres en les plaçant face à la globalité des problèmes d'implantation et de transformation du territoire et en les mettant en situation de collaboration, mais aussi de concurrence, avec les ingénieurs de haut niveau, ingénieurs topographes ou géodésiens. C'est ainsi que l'«Empire colonial » a constitué un appui non négligeable au processus de professionnalisation des géomètres, qui s'amorce en métropole au début du XX' siècle. Certes, il ne s'est agi que d'une minorité, au regard d'environ 4000 géomètres que compte alors la France, qui est regroupée dans la SNGFAT puis dans I'U GEF, autour de I'aile « modernisatrice » du mouvement corporatif agissant au sein de ces organisations. Ce courant milita activement pour le diplôme «d'ingénieur géomètre » que créa finalement l'Ecole supérieure de topographie et pour le diplôme d'Etat de géomètre expert obtenu avec le décret du 25 avril 1929. Les travaux entrepris par les géomètres aux colonies ont construit une base pour le renouvellement d'une culture technique et d'un statut socioprofessionnel, qui a contribué à faire passer ce métier, resté longtemps en lisière de l'industrialisation et figé dans l'ancestrale image de «l'arpenteur », dans le monde de l'ingénieur. 


\section{N OTES}

1 En 1905, la Société nationale des géomètres de France, d'Algérie et de Tunisie (SN GFAT) établit un accord avec un établissement privé, I'ESTP, pour un enseignement professionnel technique par correspondance. Sur les débuts de cet enseignement, cf. VACHER H., «L'Ecole supérieure de topographieet le géomètre urbain (1900-1939). Les débuts de l'enseignement technique de l'aménagement », Les A nnales de la recherche urbaine, $\mathrm{n}^{\circ} 88$, décembre 2000, pp. 121-129.

2 Sur cette question, voir HEADRICK D. R., The tools of E mpire, N.Y./ Oxford, Oxford University Press, 1981 ; The tentacles of progress, technical transfer in the Age of imperialism 1850-1940, N.Y/ Oxford, Oxford University Press, 1988.

3 CAMILLE Guy, «Etude des progrès matériels - Rapport préliminaire » in Congrès international colonial, rapports, mémoires et procès verbaux des séances, Paris, A. Challamel, 1901, p. 5.

4 E. Cheysson a notamment été à la direction des usines Schneider au Creusot, puis du service d'Economie générale et de Statistique au ministère des Travaux publics. En 1899, il est viceprésident de la Commission de nivellement de la France. II est aussi professeur d'économie politique à l'Ecole des mines. B. Kalaora et A. Savoye le présente comme un concepteur de l'ingénierie sociale, cf. Les inventeurs oubliés - Le Play et ses continuateurs; aux origines des sciences sociales, Seyssel, Champ Vallon, 1989. En 1897, E. Cheysson exposa ses idées sur «l'homme social et la colonisation », cf. VACHER H., Projection coloniale et ville rationalisée, A alborg, A alborg University Press, 2001 (2 édition).

5 Pour une analyse de cette Société entre 1895 et 1914, cf. VACHER H., « Les figures de l'ingénieur colonial à la fin du XIXe siède : la formation de la Société française des ingénieurs coloniaux et de l'Ecole spécial e des travaux publics», Le M ouvement social , n 189, octobredécembre 1999, pp. 47-65.

6 Pour une approche de ces réseaux des industries du BTP avec le parti colonial, cf. LAGANA M., Le Parti colonial français, Québec, Presses del'Université du Québec, 1988.

7 Les activités menées dans le champ colonial sont omniprésentes dans les travaux de la STP qui attribue des médailles d'honneur. Par exemple : 1877 - Colonel Roudaire, «projet de mer intérieure de l'Algérie»; 1883-J. Dupuis, « exploration du fleuve Rouge»; 1892 - Capitaine Cupet, « levés topographiques en Indochine » ; 1906 - Lieutenant Poirmeur, «travaux topographiques dans le Sud-Oranais » ; 1907 Capitaine Cottes, «travaux de délimitation de la frontière Congo-Cameroun »; 1908 - Lieutenant. de vaisseau A bel Larras, «cartes marines du Maroc »; 1910 - aux compagnies sahariennes, sous la direction du colonel Laperrine et de L. Gentil, «travaux topographiques et géologiques du Maroc » ; etc.

8 Ainsi le disimètre à collimateur facilitant les opérations de mesure de la déclivité, cf. Bulletin de la sociétéfrançaise des ingénieurs coloniaux, 1902, n²6, p. 259.

9 LEJEUNE Dominique, Les sociétés de géographies en France et I'expansion coloniale au XIX ${ }^{\circ}$ siècle, Paris, A. Michel, 1993. Parmi plusieurs travaux sur l'épistémologie de la géographie, signalons BERDOULAY V., La formation del'Ecole française de géographie (1870-1914), Paris, BN, 1981 et SOUBEYRAN O., Imaginaire, scienceet discipline, Paris, L'Harmattan, 1997. Voir aussi DORY D., «Géographie et colonisation en France durant la troisième République (1870-1940) » in PETITJEAN Patrick et al., «Science and Empires », H istorical studies about scientific development and European expansion, Dordrecht/ Boston/ London, Kluwer Academic Publishers, vol. 136, 1992, pp. 323-329.

${ }^{10} \mathrm{Cf}$. colonel Berthaut, Les ingénieurs géographes militaires 1624-1831, Paris, Imprimerie du S.G.A ., 1902.

11 Compterendu de la conférence de Delahu, in Congrès national et international des géomètres, Bruxelles, 6-10 août 1910, Bruxelles, Imp. V. Férou, 1911, pp. 67-72 $\mathrm{n}^{\circ} 131$, p. 461.

12 LECQ J., «La topographie à l'exposition coloniale », Journal des géomètres experts français, sept. 1931,

13 HOBSBAWM Eric J., L'ère des empires, 1875-1914 , (trad. française) Paris, Fayard, 1989, p. 87.

${ }^{14}$ JA CQUEMART, Paul (Ed.), Professions et M étiers, guide pratique à I'usage des familles et de la jeunesse pour le choix d'une carrière, t.1 Professions libérales, Paris, A. Colin, 1891, p. 454.

15 En 1912, les traitements pour les services topographiques sont en Tunisie de 2400 à 3600 francs pour les «géomètres » (4 classes), de 4000 à 5000 francs pour les «vérificateurs »; en Algérie, les 
« topographes ordinaires » (4 classes) gagnent de 1500 à 2400 francs, les «topographes principaux », de 1700 à 2000 francs. Cf. L'E cole chez soi, n 12, mars 1913, p. 3.

16 Journal des géomètres experts, $n^{\circ} 285$, mai 1905, p. 220.

17 Ibidem, p. 221.

18 PINÇON E., « Note sur l'Exercice de la profession de géomètre en Algérie », Compte rendu du Congrès International des géomètres tenu à Paris du 15 au 18 octobre 1926, p. 488-489, p. 488.

19/bidem, p. 489.

20 Sur la formation de I'UGEF, cf. VACHER H, Le géomètre urbain et l'urbaniste, I'Ecole supérieure de topographie de l'ESTP et I'U nion des géomètres experts français, rapport de recherche pour lePUCA/ ministère de I'Equipement, du Logement, des Transports et du Tourisme, octobre 2000.

${ }^{21}$ Sur le rôle des missions scientifiques durant cette période, cf. RIVET Daniel, Lyautey et l'institution du Protectorat français au M aroc, 1912-1925, 3 vol., Paris, L'Harmattan, 1988, t. 1, pp. 20-26.

22 Bulletin de la Société de géographie du $M$ aroc, $n^{\circ}$ 1,1916, pp. 37-38.

23 Colonel BERTHAUT, Les ingénieurs..., op. cit., p. 488.

24 Commandant Ed. de MARTONNE, «La cartographie au Maroc », La Géographie, Extrait des numéros de sept/ oct/ nov. 1921, p. 5. Edouard de Martonne était le frère du géographe Emmanuel de Martonne qui était le gendre de Vidal de La Blache. Sur les postes occupés par Ed. de Martonne, voir Bulletin périodique de la Société de topographie de F rance, décembre 1937, n 5, pp. 5-6.

25 II a été réorganisé, à Rabat, en 1919 sur le modèle du BTM.

26 Ibidem, p. 23.

27 Ainsi des photos aériennes visant à compléter la carte de reconnaissance au $1 / 200000^{\circledR}$ sont effectuées à la fin 1916 par l'escadrille de Taza. Cf., commandant Poirmeur, chef d'état-Major de Taza, « Note sur la restitution cartographique des photographies aériennes », Bulletin de la Société de géographie du M aroc, $\mathrm{n}^{\circ}$ 3, 1917, pp. 4358

${ }^{28}$ Ministère de la Défense nationale et dela Guerre-Le Service géographique del'armée - son histoire, son organisation, ses travaux, Paris, Imprimerie du SGA, 1938, p. 162.

29 Capitaine Orcel, La photo-topographie au M aroc, Conférences faites au centre de perfectionnement de M eknès, Troupes d'occupation du Maroc, état-major du général commandant en chef, mai 1918, pp. $27-28$.

30 Bulletin de la Société de géographie du $M$ aroc, $n^{\circ} 6$ \& 7, décembre 1918, p. 49.

31 En particulier les géomètres Charles Wolf et Alfred Tardif qui ont largement participé aux opérations de lotissements à Casablanca.

32 sur le système d'immatriculation et la législation de l'urbanisme, cf. VACHER H., Projection coloniale et ville rationalisée, Le rôle de l'espace colonial dans la constitution de l'urbanisme en France, 1900-1931, Aalborg, Aalborg University Printing House, vol. 33, 2001.

33 L'application partielle du système «Torrens » venant compléter les dispositions de la loi de 1873 fit l'objet d'études détaillées, cf. DAIN Alfred, Le système Torrens - D e son application en Tunisie et en A lgérie Rapport à $M$. Tirman gouverneur général de l'A Igérie, suivi d'une traduction de l'A ct Torrens et de la loi foncière tunisienne du 5 juillet 1885, Alger, Adolphe Jourdan, 1885.

34 Sur cette question, voir la revue éditée par J.-L. Sanguet, La Réforme cadastrale ; HERBIN R. et PEBEREAU A., Le cadastre français, Paris, Francis Lefevre, 1953 ; CHEYSSON E. et SAINT-PAUL M., La question technique du cadastre en France, Paris, Paul Dupont, 1901.

35 GA LLIENI G., « Sur la situation générale de Madagascar », Journal officiel, 2 juin 1899, p. 3722.

36 Baronnat, «Immatriculation foncièreen Tunisie, dela nécessité d'une loi immobilière», Journal des géomètres experts et topographes français, juin 1933, n 152, pp. 297-304, p. 304.

37/ Ibidem, p. 299. 


\section{p. 818. \\ ${ }^{38}$ Codes et lois en vigueur dans le Protectorat français du M aroc, Paris, Imprimerienationale, 1925, vol. 1, \\ 39 Par arrêté «viziriel » du 26 octobre 1915; I'arrêté« viziriel » du 20/ 1/ 1917 portecréation d'un corps} spécial d'agents du service de la Conservation de la propriété foncière.

40 L'immatriculation présente «cet immense avantage d'arrêter définitivement, après toute la publicité et les garanties nécessaires, les revendications sans fin autorisées par la loi musulmane en matière immobilière ». Villes et tribus du M aroc, D ocuments et Renseignements, publiés sous les auspices de la Résidence générale. vol. IV, t. II, Paris, E. Leroux, 1919, p. 161.

41 RIVET Daniel, Lyautey ..., op. cit. , t. 1. pp. 230-232.

42 En 1921, le service de la Conservation est constitué d'une direction et conservation à Rabat dirigé par Marc Roussel, d'une conservation de Casablanca, dont Roland est conservateur ; d'une conservation d'Oujda dirigée par M. Nerrière, conservateur. Cf.,A nnuaire général du M aroc de1921, Casablanca, L. Guigues, pp. 76-77.

43 Journal des géomètres experts français, 1922, p. 219.

44 Pour exemple, figurent parmi les plans exposés «un plan d'immatriculation au $1 / 100$ et un plan rural au 1/ 5000 ; une nappe foncière au 1/ 50000 de la Conservation de Rabat; le plan d'ensemble au 1/ 1000000 des lots de colonisation et des immeubles collectifs du Maroc ; un titre de propriété au 1/ 10000 de la ferme de Polygnac; un levéau 1/ 10000 des lots de colonisation del'oued Fès », cf. Journal des géomètres experts français, $n^{\circ} 131$, septembre 1931, p. 466.

45 Ces textes sont : ledahir du 16 avril 1914 portant sur les alignements, les plans d'aménagements et d'extensions des villes, les servitudes et les taxes de voiries; le dahir du 31 août 1914 donnant aux pouvoirs publics les moyens de procédure et d'expropriation «dans le cadre des plans d'aménagement et d'extension des villes, servitudes et taxes de voirie » et quelques dispositions anti-spéculatives ; le dahir du 10 novembre 1917 qui complète celui du 16 avril 1914 en introduisant les associations syndicales de propriétaires urbains (ASP).

46 Journal des géomètres experts, 1907, p. 21 \& pp. 60-61.

47 Ces différents thèmes font l'objet de nombreuses notes dans le J ournal des géomètres experts de ces années d'avant-guerre.

48 VACHER H., L'EcoleSupérieure ..., op. cit., p. 123.

49 Bulletin de I'A ssocation amicale..., op. cit., 1914, p. 12.

50 Données obtenues à partir du dépouillement du Bulletin del'A ssociation amical e de I'ESTP entre 1912 et 1920. Précisons que ces annonces, pendant la guerre, concernent presque exclusivement le Maroc ; elles représentent quelques dizaines d'offres sur près des 900 diffusées par le bulletin en 1916-1919.

${ }^{51}$ Cf. VACHER H., Le géomètre urbain..., op. cit., p. 46. En ce qui concerne l'enseignement de Danger, cf. DANGER R., Cours de topométrie urbaine, lever des plans de ville. Paris, LET/ ESTP, 1921 ; cf., Bulletin des géomètres experts français, $n^{\circ} 11$, nov. 1921; cf. CULOT M. et THIVEAUD J.-M., A rchitectures françaises outre mer. Liège, Mardaga/ IFA, 1992, p. 387. II ne subsiste aucune trace de l'enseignement de René Danger à Casablanca en 1918-1919 dans les fonds disponibles aux Archives d'A rchitecture de I'IFA. La concomitance entre la reprise d'un enseignement spécialisé en topographie à l'ESTP et l'organisation d'une formation à Casablanca nous mène à faire l'hypothèse d'une mission marocaine de Danger, probablement avec la casquette de I'ESTP, pour mettre sur pied cet enseignement au Maroc. Pour I'annonce de la création d'une école de géomètreà Casablanca, cf. Bulletin de l'A ssociation amicale, n 120, avril-mai 1919, p. 197.

52 Journal des géomètres experts français, 1920, n 570, p. 479

53 Bulletin officiel du Protectorat du M aroc, n454, 5juillet 1921.

54 Journal des géomètres experts français, 1922, p. 62.

55 Journal des géomètres experts français, 1922, p. 273.

56 Bulletin de l'A ssociation amicale, $n^{\circ} 136$, février 1922, p. 37.

57 Journal des géomètres experts français, 1921, n 11, p. 307. 
58 Bulletin del'A ssociation amicale..., n 120, avril-mai 1919, p. 197.

59 II s'agit de : A ubert, Buan, Cousinet, Dupuy, Lapierre, Massol, Reno, Sanmarti, Tardif, Wolff. Cf. A nnuaire général du M aroc. 1918-1919. Casablanca., p. 226.

60 Cf. A nnuaire général du M aroc de 1921, Léon Guigues, p. 105.

61 « Les plans de ville au Maroc », Journal des géomètres experts, janvier 1918, n543, p. 17-18.

6 LASSALLE J., «Remembrements et redistributions urbaines », L'Ingénieur-Constructeur, $n^{\circ} 133$, novembre 1921, pp. 521-545, p. 521.

63 Le remembrement est intégréà la législation métropolitaine de l'aménagement avec les décrets-lois de 1935 sur l'aménagement de la région parisienne. L'ingénieur des Ponts et chaussées Edouard Joyant diffuse pendant toute l'entre-deux-guerres le mode de fonctionnement des associations syndicales de propriétaires (ASP), dont il a suivit la miseen place au Maroc en tant quedirecteur à la direction générale des Travaux publics. Ces réalisations servent d'illustration aux cours qu'il donne à l'Ecole supérieure de topographie dans les années vingt. Pour la contribution d'E. Joyant, voir notre mémoire de recherche, Rationaliser la ville, L'Ecole spéciale des travaux publics de Léon Eyrolles et la formation aux techniques et disciplines de l'aménagement (1898-1939), rapport de recherche pour le PU CA, Paris, 1998, pp. 86-87. pp. 331-340.

64 DANGER R., « Le géomètre en Syrie », Journal des géomètres experts français, n 105, juillet 1929,

66 On peut définir la tachéomètrie comme la technique de levé des plans à l'aide du tachéomètre, instrument qui permet de mesurer simultanément les hauteurs et les distances horizontales.

66 DURAFFOURD C., «La technique des travaux du cadastre en Syrie», Journal des géomètres experts français, $n^{\circ} 107$, septembre 1929, pp. 436-444, p. 436.

67 Ibidem, p. 444.

68 Journal des géomètres experts français, n 112, février 1930, p. 78-79.

69 LECQ J., «L'art du géomètre à la coloniale», Journal des géomètres experts français, $n^{\circ} 133$, novembre 1931, p. 579-590.

70 On la voit se mettre en place à Paris entre 1905 et 1914. Rappelons que la SNGFAT avait espéré conclure un accord en 1903 avec le ministère de l'Agriculture pour la création d'une section de géomètres topographes au sein del'Institut agronomique de Paris, mais le projet fut sans lendemain. 

CHAPITRE 3

\section{PROFESSIONNALITÉS CONTEM PORAINES}





\section{Evolution du profil de l'ingénieur algérien : du « technicien » au « développeur »?}

Hocine KHELFAOUI

Le but de cette contribution est d'esquisser les conditions d'émergence d'un nouveau profil d'ingénieur en Algérie. Ce profil se caractérise essentiellement par une évolution des tâches et des missions traditionnellement attachées à l'ingénieur en Algérie. Jusquelà confiné dans des tâches de fonctionnent des équipements ou promu dans des fonctions de gestion administrative, l'ingénieur algérien est de plus en plus engagé dans des activités associées à l'innovation. Cette perspective annonce une refonte de son rapport à l'entreprise et aux institutions de savoirs (universités, centres de recherche, etc.).

Ce constat justifie que l'on s'intéresse aux divers processus qui ont contribué à l'émergence de l'ingénieur développeur en Algérie. Après une esquisse rapide du contexte général qui a favorisé ou accompagné l'apparition de ce nouveau profil, on s'intéresse, dans un premier temps, aux impacts que pourrait produire l'affirmation de I'ingénieur développeur sur le développement de l'entreprise et des institutions de savoirs. Dans un second temps, nous traitons des nouvelles articulations qui tendent à se dessiner entre les ingénieurs et les chercheurs.

\section{LE CONTEXTE GÉNÉRAL}

Deux grandes périodes caractérisent l'ingénieur algérien depuis 1962. Chacune de ces périodes le distingue par une place particulière aussi bien dans l'entreprise que dans le système éducatif. De l'indépendance à la fin des années 1980, les stratégies de formation des ingénieurs ont été déterminées par des logiques plus politiques que techniques et économiques. Autrement dit, les politiques de formation ont été guidées par des «calculs » et des objectifs partisans plus que par des ambitions de performance économique et de maîtrise sociale des savoirs. La grande hiérarchisation des ingénieurs 
au sein del'entreprise et son effritement en tant que groupe professionnel renvoyai ent à la structuration du système algérien de formation, elle-même expression des différents clivages qui traversaient la classe dirigeante algérienne. Chaque courant politique se faisait, en quelque sorte, représenter au sein de l'entreprise par une catégorie d'ingénieurs formée par ses soins et dont la carrière dépendait de son influence dans la sphère du pouvoir.

Les ingénieurs s'inscrivaient alors dans un rapport clientéliste avec les différentes factions dirigeantes ; ils apparaissaient, à leur corps défendant, bien plus comme le prolongement de ces pouvoirs au sein des grandes entités économiques publiques que comme des catégories professionnelles au service des entreprises. Sur le terrain, et selon sa proximité avec ces pouvoirs, l'ingénieur était soit cantonné dans des tâches de fonctionnement des équipements importés, soit promu dans des fonctions de gestion bureaucratique. Dans le premier cas, il se trouvait confronté aux techniciens, et parfois même aux ouvriers expérimentés qui ne voyaient pas quel pouvait être l'apport spécifique de sa qualification. Alors qu'ils le percevaient comme un inventeur et un innovateur, l'ingénieur venait les concurrencer, mal d'ailleurs, dans leur propre domaine professionnel, celui de la technicité et du savoir-faire. Dans le second cas, il renonce à ses qualifications initiales pour épouser, le plus souvent, celles d'un simple donneur d'ordres venant de l'extérieur de l'entreprise.

Ce système est entré en crise à la fin des années 1980, autorisant de ce fait l'émergence de ce nouveau profil d'ingénieur. Le contexte général qui a donné naissance à «l'ingénieur de fonctionnement » a radicalement changé, passant sans transition du « tout pour l'Etat» au «tout pour le marché ». Avec la fin des subventions étatiques ${ }^{1}$, I'entreprise n'a plus les moyens d'importer ses équipements ; elle fait face à un marché national totalement livré à la concurrence internationale $e^{2}$. Elle doit désormais innover et compter sur ses propres forces pour améliorer ses performances. Le capital de savoir et de savoir-faire accumulé pendant les années d'industrialisation a certainement contribué à cette maturité.

Le renouveau que connaît depuis un peu plus de dix ans ce groupe professionnel vient essentiellement de l'entreprise, tandis que le premier type d'ingénieurs a été forgé, pour l'essentiel, par les pouvoirs publics et les institutions de formation, pour être livré « clés en main » aux «utilisateurs ». Rompant avec la figure ambivalente de l'ingénieur tantôt technicien tantôt gestionnaire, l'ingénieur développeur se fixe des objectifs d'ingénierie et d'innovation technique. La professionnalisation des ingénieurs cesse 
d'être ce qu'elle était jusquelà, c'est-à-dire conçue et façonnée par le système de formation et les pouvoirs publics, pour devenir progressivement le fait de l'entreprise.

Dans ce nouveau cadre, l'ingénieur conforte sa position professionnelle face à celle des « administratifs » dont le pouvoir d'attraction sur les catégories techniques s'est ainsi sensiblement réduit. Contrairement à la première période, le jeune ingénieur préfère désormais investir dans les carrières techniques plutôt qu'administratives ${ }^{3}$.

De telles transformations ne peuvent rester sans impact sur le fonctionnement interne de l'entreprise et sur les relations qu'elle entretient avec les institutions de formation et de recherche.

\section{L'IMPACT DES TRANSFORMATIONS DE LA PROFESSION D'INGÉNIEUR SUR LE FONCTIONNEMENT DE L'ENTREPRISE}

Avec la revalorisation des activités de création, les relations de pouvoir au sein de I'entreprise sont fondamentalement modifiées. Les tentatives de recentrage des activités sur l'innovation ont eu des incidences majeures sur les fonctions hiérarchiques. Alors que l'innovation implique la maîtrise du changement, le système de pouvoir mis en place et consolidé depuis les années 1970 est conçu en fonction de l'exigence de la stabilité. En outre, si les hiérarchies existantes reposent sur des pouvoirs d'ordre, la gestion de l'innovation s'appuie sur des pouvoirs d'expertise et de compétence technique à tous les niveaux. Les formes de relations professionnelles s'en trouvent modifiées, et les valeurs du travail affectées. Les notions de compétence et de qualification sont passées d'une conception «substantialiste » à une conception «relationniste »4. Dans l'entreprise publique algérienne, les nouvelles formes de compétence technique et sociale n'ont pas seulement à s'imposer face aux formes anciennes de qualification. Elles doivent également composer avec les rapports sociaux locaux, fortement imprégnés de considérations familiales, régionales, etc., en somme, avec toutes sortes de liens sociaux, issus de pouvoirs exogènes dans leur essence, et qui ne convergent pas nécessairement avec la logique de l'entreprise telle qu'elle s'exprime. II est ainsi possible d'affirmer l'existence d'une relation directe entre le mouvement de renouvellement managérial, avec ses incidences relationnel les et organi sationnelles, et le processus d'innovation, avec ses effets sur la compétitivité des entreprises.

On peut observer une relation quasi directe entre la réforme de l'entreprise, dans le sens d'une plus grande participation des partenaires sociaux, et les efforts de démocratisation au sein de la société. L'entreprise algérienne tente, avec toutes les 
pesanteurs de son environnement, de s'amarrer à un renouveau managérial qu'elle a tôt perçu mais jamais pu appliquer. Les tentatives d'introduction de ces techniques managériales y sont pratiquement contemporaines de leur adoption par leurs homologues européennes. Certes, leur échec relatif (rapporté à leur succès dans d'autres contextes socio-politiques) s'explique par la résistance des bureaucraties de tutelle, mais aussi d'une partie des cadres. Ces derniers y voient une remise en question des critères de sélection qui ont présidé à leur propre ascension professionnelle.

\section{L'IMPACT SUR LES INSTITUTIONS DE FORMATION}

Cette évolution a également diversement touché les institutions de formation (instituts technologiques, universités, écoles, etc.). Durant la première période, le cloisonnement de ces institutions n'a guère favorisé le contact avec l'environnement industriel $^{5}$. Longtemps, il a été difficile aux élèves ingénieurs, comme aux enseignants, de mesurer l'ampleur du décalage entre l'étape de la formation et celle de la professionnalisation, entre les enseignements théoriques dispensés et les compétences exigées sur le terrain. Les formateurs restaient dans une large mesure fixés sur une demande industrielle dont les contours avaient été dessinés pendant les années 1960 et 1970, notamment lors des débats qui ont précédé et nourris les deux grands séminaires nationaux sur La Formation des Cadres pour le D ével oppement.

L'ampleur des besoins avait al ors conduit à fixer aux institutions de formation des missions en quelque sorte industrielles : elles devaient non seulement former « massivement », mais aussi dispenser des formations «directement opérationnelles », c'est-à-dire former des cadres ayant acquis au sein des institutions de formation des qualifications relevant du monde de l'industrie. De la même manière, l'ampleur de I'anal phabétisme des salariés avaient imposé aux entreprises publiques d'assumer des fonctions d'enseignement et de formation de base. II en a résulté, au sein du système mis en place, une confusion de prérogatives et de chevauchement des rôles entre formateurs et producteurs, et qui s'est prolongée pendant toute la première période. Mais si les entreprises ont pu se dégager de ce carcan, il n'en a pas été de même pour les institutions de formation, qui commencent à peine à connaître une évolution similaire en essayant de s'ajuster aux évolutions. Le décalage entre ces deux institutions a engendré, au cours des années 1990, un dialogue de sourds aisément observable lors des forums organisés autour du thème, pourtant largement rebattu depuis l'indépendance, de «la relation université-entreprise $»^{6}$. A priori, tout le monde s'accorde en parlant de formation 
technologique, d'adéquation formation-industrie, de performance, d'excellence, etc. Mais à y regarder de plus près, on peut se rendre compte que les convergences ne sont qu'apparentes. En effet, s'il y a consensus autour des thèmes, il en est autrement de leurs contenus. Si le même vocabulaire est utilisé, les mots revêtent des significations différentes pour les uns et pour les autres. Pour les entreprises, une formation d'excellence signifie esprit d'initiative et d'innovation, aptitude à la mobilité et capacité de s'adapter au changement technologique. Pour les formateurs des universités et des instituts, restés fixés sur le modèle des années 1970, une formation d'excellence signifie toujours une spécialisation sans cesse plus poussée et des aptitudes à maîtriser le fonctionnement de l'outil de production.

Ce quiproquo s'est maintenu pendant longtemps, notamment à cause de I'insuffisance de contacts entre les acteurs directs de la formation et ceux de l'industrie, c'est-à-dire entre les enseignants et les ingénieurs d'entreprises, et en raison de leur exclusion du processus d'élaboration de cette relation. Cet ancrage dans le passé a été particulièrement présent dans les instituts technologiques. Forts d'une identité institutionnelle perçue comme immuable, nombre de dirigeants de ces établissements n'envisageaient pas que la formation dispensée puisse entretenir un rapport particulier et évolutif à l'industrie. Cette identité était considérée non comme une dynamique d'adaptation permanente à l'évolution du secteur industriel, mais comme une sorte de constante, marquant définitivement la nature même des instituts. Cette constante prenait ici la forme d'une quête permanente de spécialisation des cursus, une sorte de fuite en avant dans la spécialisation : à des entreprises de plus en plus insatisfaites, les instituts proposaient des formations de plus en plus spécialisées. II a fallu que ces établissements changent de secteur, de statut, de profil d'enseignants et de dirigeants pour qu'une évolution dans les cursus de formation fixés il y a trente ans commence à se dessiner. Aiguillonnées par la nécessité de survivre, les entreprises ont été les premières, par comparaison aux établissements de formation, à avoir tiré des leçons de l'expérience antérieure. De nombreuses divergences de perceptions et d'intérêts apparaissent en effet lorsque l'on analyse les discours : alors que les enseignants et les responsables des universités recherchent toujours la protection de l'Etat en revendiquant la mise en place d'une relation de type institutionnel, fondée sur des lois et des règlements, les entrepreneurs insistent sur la communication informelle et la connaissance mutuelle des deux partenaires. Pendant que les entreprises parlent de survie, les universités se contentent de tenir des discours emphatiques à l'instar de ceux qui évoquent la nécessité «d'affronter les défis et les enjeux » du nouveau millénaire. 


\section{LA RECHERCHE-DÉVELOPPEMENT : UNE NOUVELLE DEMANDE DE L'ENTREPRISE ?}

Le style de formation, tel qu'il est formulé par l'entreprise ou mis en œuvre par les différentes institutions d'enseignement, a connu une importante mutation au cours des années 1990. On assiste alors au passage d'un type de besoins à un autre : le premier est formulé en terme de qualifications nécessaires au fonctionnement des équipements ; et le second, en terme de recherche de qualifications nécessaires à l'innovation et au renouvellement technologique. Contrairement à la première configuration, le nouveau profil n'est pas défini au niveau de l'Etat et imposé sous forme de lois et de règlements aux deux partenaires. L'évolution en question est le résultat de contacts répétés et de négociations directes entre l'entreprise et les institutions de savoirs, qui incluent de plus en plus les centres de recherche, et non celui d'une politique volontariste central isée. Elle est aussi le résultat d'un intérêt croissant des entreprises pour ce type de dialogue.

Lors de ces rencontres, la recherche-développement tient une place de plus en plus importante dans le discours des dirigeants d'entreprises publiques comme privées : elle est présentée comme une activité nécessaire à leur survie. On peut même constater que des réunions dont l'ordre du jour porte sur les problèmes de formation sont détournées et transformées en débats sur la recherche-développement. Ainsi, les discussions ne visent plus à définir les capacités qui devraient permettre au jeune ingénieur d'être « opérationnel » dès les premières semaines de son embauche, mais plutôt son aptitude à faire face au changement technologique et à l'innovation. Dans la plupart de leurs interventions, les responsables d'entreprise évoquent la recherchedéveloppement en tant que fonction vitale assurant la pérennité de l'industrie algérienne. Ils la placent devant la qualité, le marketing et l'analyse économique, autres fonctions qui reviennent souvent dans les nouveaux discours des managers. On pourrait, bien sûr, voir dans ces propos un simple discours ${ }^{7}$. Néanmoins, ils reflètent de réelles interrogations chez les responsables des entreprises des secteurs public et privé ${ }^{8}$. En outre, cette nouvelle tendance se concrétise par la création de nombreuses structures de recherchedéveloppement au sein même des entreprises, et par l'augmentation sensible des budgets qui leur sont consacrés. Les gestionnaires affirment, sur un ton parfois pathétique, que I'entreprise doit «relever des défis qu'elle n'a pu surmonter en période "faste", comme le développement de produits nouveaux ».

La maîtrise des savoirs, entendue au sens d'innovation, est ainsi devenue une des préoccupations essentielles des entreprises au cours de la dernière décennie. Beaucoup de projets de recherche initiés par des laboratoires de centres de recherche ou 
d'universités sont soutenus par des entreprises. Certes, cette situation converge avec I'orientation originellement donnée à la science par les pouvoirs publics, dès le début des années $1970^{9}$; mais elle ne lui doit pas grand chose. Elle est plus le résultat d'une évolution interne des partenaires, liée à des contextes socio-politiques particuliers. Elle est aussi liée, dans une certaine mesure, à I'union de la science avec l'industrie, que l'on peut universellement observer depuis une dizaine ou une quinzaine d'années.

La nouvel le relation université-entreprise s'inscrit dans le sillage de cetteévolution, désormais prédominante dans les pays les plus industrialisés. En Algérie, elle s'est accélérée ces dernières années en raison de l'importance que les chercheurs accordent au fait d'avoir des liens avec l'entreprise, celleci étant devenue leur principal pôle d'attraction, à défaut d'autres centres d'intérêts. Marginalisé et relégué par le politique, les scientifiques se sont tournés presque tout naturellement vers les entreprises. L'insertion des chercheurs algériens dans les réseaux internationaux reste relativement faible, la guerre qui sévit dans le pays (avec ses prolongements internationaux) ayant contribué à son isolement ${ }^{10}$. Le départ de la plupart de chercheurs et des ingénieurs de la première génération a considérablement réduit le poids du paradigme académique, faisant place à des jeunes dont la formation est moins portée sur les sciences dites pures. A ces considérations, viennent s'ajouter les conditions de vie des chercheurs. Le revenu attaché aux fonctions scientifiques est dérisoire, contraignant les meilleurs chercheurs, parmi ceux qui restent attachés à leur vocation première, à rechercher des compléments de revenus dans l'entreprise. Cette situation a aussi poussé la majorité des chercheurs vers des activités sans rapport avec le savoir, commerce et petits business de toutes sortes.

C'est dans ce contexte que certaines entreprises tentent de tisser des liens avec certains chercheurs rattachés à des universités ou à des centres de recherche. Elles suscitent cette participation d'autant plus aisément que les scientifiques, renonçant à une autonomie socioprofessionnelle longtemps ardemment défendue, se tournent de plus en plus vers I'industrie. En fait, les scientifiques attendent non seulement de l'entreprise qu'elle finance leurs projets ou qu'elle en soit le lieu d'application d'éventuels résultats, mais aussi qu'elle contribue à l'amélioration de leurs conditions de vie matérielles, largement dégradées cette dernière décennie. Par ailleurs, leurs difficultés matérielles et sociales les conduisent souvent à passer des contrats sans relation avec la recherche scientifique.

Après plus de deux décennies de conflits et d'ignorance mutuelle, de relations distantes et fondées sur l'indépendance des deux activités, l'entreprise et les institutions de savoirs tentent de systématiser leur partenariat. Ce dernier est construit non pas 
autour de la fonction de formation, mais essentiellement autour de l'intégration de la recherche dans l'entreprise. La recherche, qui était jusquelà surtout le fait d'institutions ad hoc ou d'individualités marquantes, a tendance à devenir, en pénétrant l'entreprise et d'autres catégories socioprofessionnelles, une création collective, un mouvement de société auquel participe des catégories sociales de plus en plus larges ${ }^{11}$. Banalisés dans leur fonction symbolique, les «scientifiques » ne sont désormais qu'une catégorie socioprofessionnelle parmi bien d'autres.

\section{L'INGÉNIEUR ET LE CHERCHEUR : UNE NOUVELLE RELATION ?}

De ce contrat d'intérêt entre l'entreprise et la science est née la figure du « chercheur-développeur ». Ce dernier est issu de la fusion entre l'ingénieur d'industrie - passé du statut d'ingénieur de fabrication à celui d'ingénieur d'innovation - et le scientifique. De son côté, ce dernier est passé du statut d'universitaire à celui, plus concret, de chercheur dont les activités s'inscrivent de plus en plus dans des projets industriels. L'entreprise joue ainsi un rôle notable dans le passage d'une recherche individuelle et disciplinaireà une recherche en équipes et réseaux pluridisciplinaires. Ses activités étant transversales à plusieurs secteurs ou spécialités, les cloisonnements disciplinaires ne répondent plus aux mouvements actuels d'une science tournée vers les besoins de l'industrie. Quelles sont cependant les modalités sociales d'une telle coopération?

Désormais, pour le chercheur, la possibilité d'intéresser les entreprises à une éventuelle mise en application industrielle des résultats de son projet, est le meilleur moyen de valoriser son travail et surtout de se faire reconnaître. Pour nombre de chercheurs interviewés, une reconnaissance industrielle est socialement plus valorisante, et donne lieu à une meilleure considération, même au sein de la communauté des pairs. Cette tendance implique des changements dans les modes de légitimation sociale des savoirs qui, jusque-là, plaçaient la connaissance abstraite au sommet de la pyramide des savoirs. De même, elle révèle l'apparition de nouveaux styles de sciences au sein de la communauté scientifique. Cette quête de reconnaissance par l'entreprise est aussi à mettre en relation avec l'incapacité des scientifiques algériens à s'ériger en groupe « autosuffisant ». Leur faiblesse numérique et organisationnelle et l'absence relative de soutien étatique $\mathrm{e}^{12}$ engendrent chez eux un sentiment de dépendance à l'égard d'autres formes de reconnaissance et de légitimation. Celles qui viennent de l'entreprise sont appréciées, même si elles ne s'accompagnent pas d'avantages matériels. L'évaluation par 
l'entreprise entre de plus en plus en compétition avec celle, classique, des pairs. Les chercheurs et leurs institutions sont plus soucieux de convaincre l'entreprise que leur propre conseil scientifique. Pour ce faire, ilsn'hésitent pas à associer des ingénieurs à leur projet et à s'engager contractuellement dans des missions parfois hasardeuses.

Ce processus de valorisation, qui met le chercheur en contact direct avec le terrain, tend à déterminer, dans une large mesure, les modalités de son insertion dans le champ de l'entreprise. II détermine aussi, à une échelle plus large, le processus d'émergence et de reconnaissance de la communauté scientifique al gérienne. La reconnaissance sociale du chercheur et de la communauté des chercheurs (donc leur statut de groupe professionnel distinct) dépend de leur capacité à réaliser ce produit et à le vendre, c'està-dire à le faire accepter par l'entreprise. Or, l'application de ce « contrat » se heurte à une difficulté : celle du contact avec l'autre partenaire de l'innovation qu'est l'ingénieur, et dont dépend le succès aussi bien de la recherche que de l'exploitation de ses résultats. D'une grande complexité, cette mise en relation touche au point faible du système socioorganisationnel algérien, à savoir ses capacités de communication et son autonomie à l'égard du champ politique. D'une part, l'aboutissement de ce contrat est tributaire du mode de coopération qui s'établit entre les acteurs de cette innovation, processus social dont l'aboutissement dépend d'un grand nombre d'acteurs, au premier rang desquels figurent les ingénieurs et les chercheurs. S'agissant ici de deux groupes professionnels autonomes, l'acte de coopérer dépend du respect de l'identité de chacun et de leur complémentarité dans l'action. La capacité des ingénieurs à maîtriser l'environnement scientifique et technique, à coopérer et à échanger, sont autant de critères d'insertion au sein de la communauté scientifique nationale et internationale. D'autre part, la mise en contact de deux groupes sociaux représentant des enjeux scientifiques et techniques importants implique la mise en place des conditions d'un dialogue social permanent, fondé sur l'autonomie des acteurs, sans qu'il soit politiquement médiatisé. En outre, leur association dépasse le cadre étroit des deux groupes professionnels dans la mesure où elle conduit à une association avec de grands secteurs d'activité, tels que l'économie, l'éducation et la recherche. Or, dans le contexte algérien, un tel enjeu ne saurait laisser le gouvernement indifférent. Celui-ci avait auparavant toujours contrôlé cette relation en I'institutionnalisant et en rendant impossible tout contact sans un passage obligé par les rouages de la bureaucratie. Sachant que rien de nouveau ne s'est produit dans la nature profonde du système politique al gérien, toujours fondamentalement rentier et policier, il est peu probable qu'il laisse une telle alliance s'effectuer sans lui. 


\section{L'INGÉNIEUR : UN ÉLÉMENT CONSTITUTIF DE LA COMMUNAUTÉ SCIENTIFIQUE ?}

En raison des réseaux de communication qui le relient de plus en plus à l'environnement scientifique, on peut penser que l'ingénieur algérien devient partie prenante de la communauté scientifique nationale et internationale. L'articulation de son activité professionnelle à celle du chercheur participe de cette insertion. Réci proquement, il est tout à fait possible de penser que le scientifique est en train de s'incorporer dans la rechercheindustrielle.

Cette position particulière, à cheval entre plusieurs secteurs d'activité, distingue la communauté scientifique des autres groupes professionnels, car elle transcende I'appartenance organique des chercheurs, voire leur nationalité. A la base de l'insertion de l'ingénieur dans la communauté scientifique, on peut trouver des activités propres au chercheur, telles que la participation aux séminaires et colloques, le parrainage de thèse ou la participation à des jurys, etc. Qui plus est, il semble que la participation aux manifestations scientifiques soit plus fréquente chez les ingénieurs que chez les autres scientifiques du fait de moyens financiers supérieurs. II est même courant de voir d'éminents scientifiques étrangers, invités par des opérateurs privés, donner des conférences devant un parterre non pas de scientifiques et de chercheurs, mais d'ingénieurs et de dirigeants d'entreprises.

En même temps que le chercheur part en quête d'une reconnaissance de I'entreprise, l'ingénieur opère un glissement vers des activités de recherche et d'innovation, et s'estime partie intégrante dela communauté scientifiqueet technique. En revendiquant, son appartenance à la communauté scientifique, lechercheur (d'université ou d'entreprise) exprime la supériorité de sa filiation communautaire par rapport à sa filiation organique. Cette identification des chercheurs à leur communauté plutôt qu'à l'organisme employeur implique pour eux une grande capacité d'ouverture et de maîtrise de l'environnement social. Elle en fait également des agents essentiels parmi ceux qui contribuent à mettre fin aux institutions forteresses et autosuffisantes que sont encore I'Université et l'Entreprise, deux mondes jusqu'à ces dernières années fermées aussi bien l'un à l'autre qu'à leur environnement.

Naguère, le succès de toute politique de coopération avec l'environnement passait par un mode d'articulation tenant compte du respect de l'identité professionnelle des partenaires, représentée alors par l'ingénieur d'industrie et l'enseignant d'université, leur identité catégorielle étant largement façonnée par leur appartenance organique. Dans les configurations socioprofessionnelles qui se dessinent, l'« identité » et la «culture » des 
deux partenaires tendent à se fondre, sans se diluer complètement, dans une sorte de sentiment d'appartenance à une même communauté scientifique. Toutefois, cette tendance se heurte à plusieurs obstacles, dont le plus important réside dans la difficulté de valoriser les résultats de la recherche. Déjà en eux-mêmes relativement faibles, les résultats qui arrivent au stade de la valorisation commerciale sont infimes pour ne pas dire inexistants. Malgré l'existence d'un tissu industriel non négligeable, la maîtrise de I'aval constitue un véritable blocage. Toutes les institutions de recherche, qu'elles s'insèrent dans une université, dans un centre de recherche ou dans une entreprise, se heurtent à la difficulté de franchir le cap des prototypes de laboratoire pour aller vers la fabrication en série.

Au-delà des discours récurrents autour de la relation recherche-entreprise, peu de mesures sont réellement prises pour fluidifier la communication au quotidien entre chercheurs et ingénieurs ou pour investir dans des projets pilotes et d'essais. Au scepticisme qui pèse sur la capacité de la recherche al gérienne de parvenir à des produits qualitativement compétitifs, vient s'ajouter le manque de confiance des entreprises dans leur aptitude à fabriquer en série des produits nouveaux, techniquement fiables et économiquement viables. En fin de compte, tout se passe comme si les activités scientifiques «avaient pour seule finalité de démontrer les capacités du chercheur al gérien à produire de la "science" sous la forme de savoir abstrait, sans se soucier de ses retombées sur le dével oppement social », constate un chimiste de l'Entreprise nationale des détergents (ENAD), implantée à Lakhdaria ; celui-ci déplore par ailleurs les faibles moyens mis à sa disposition pour mener à bien des expériences destinées à mettre au point un nouveau détergent qui serait selon lui plus efficace que la marque A riel.

Ainsi, la recherche al gérienne, qu'elle s' effectue dans une institution scientifique ou dans une entreprise, s'arrête presque toujours au stade du dépôt de brevet. Un document éditéen 1998 par le ministère de l'Industrie et dela Restructuration ${ }^{13}$ fait état d'un certain nombre de brevets déposés par des centres de recherche auprès de l'Institut national de la propriété industrielle (INAPI). Ainsi, le Centre de développement des énergies renouvelables (CDER) et le Centre de développement des technologies avancées (CDTA) ont respectivement déposé six et cinq brevets. L'INAPI a effectué une enquête sur le potentiel d'innovation du secteur industriel auprès de 26 entreprises publiques ou privées qualifiées de fiables. L'enquête a permis d'identifier 244 innovations se répartissant comme indiqué dans le tableau 1 : 
Tableau 1. Type d'innovations recensées

\begin{tabular}{|l|c|}
\hline \multicolumn{1}{|c|}{ Type d'innovation } & Nombre \\
\hline Solution nouvelle d'un problème technique & 50 \\
\hline Amélioration ou rationalisation d'un produit existant dans l'entreprise & 52 \\
\hline Amélioration ou rationalisation d'un procédé existant dans l'entreprise & 38 \\
\hline Innovation d'un produit n'existant pas dans l'entreprise & 75 \\
\hline Innovation d'un procédé n'existant pas dans l'entreprise & 21 \\
\hline Autre type d'innovation spécifique & 08 \\
\hline Total & $\mathbf{2 4 4}$ \\
\hline
\end{tabular}

Source : Ministère de I'Industrie et de la Restructuration, Développement des innovations technologiques en A lgérie, novembre 1998, 58 pages.

Toutefois, même les améliorations ou les inventions réal isées au sein des entreprises, recensées dans ce tableau comme étant des innovations, arrivent rarement au stade de la fabrication industrielle, et encore moins à celui de l'exploitation commerciale. De multiples raisons d'ordre technique, organisationnel ou managérial peuvent être évoquées pour expliquer cet état de fait : l'incapacité des entreprises à modifier les équipements et l'organisation pour les adapter aux exigences de produits ou procédés nouveaux, les intérêts et les rapports de force de diverses natures liés au système d'entreprise existant, I'importance des coûts financiers, l'absence « d'esprit d'entreprise» chez les managers, etc. A titre d'exemple, sur les treize inventions protégées par I'INAPI et déposées par la direction de la recherche appliquée de l'entreprise nationale de sidérurgie (SIDER) entre 1987 et 1996, cinq seulement ont atteint le stade de l'exploitation industrielle en 2000. Le tableau des inventions protégées par I'INAPI fait ressortir la situation suivante : on constate que six inventions sur treize sont arrivées au stade de l'exploitation industrielle, et ont donc donné lieu à une innovation au sens de Schumpeter, c'est-à-dire un produit nouveau commercialisable. Compte tenu de la difficulté de traverser toutes les étapes, complexes et laborieuses, qui mènent de la découverte à l'innovation, cet aboutissement constitue en soi une performance. Parmi ces innovations, trois ont donné lieu à des créations d'entreprises nouvelles, les filiales CODESID, REFRACTAL et ENSID ; une quatrième a donné lieu à l'octroi d'une licence d'exploitation en faveur d'une entreprise tiers (l'Entreprise communale des travaux d'Annaba), deux autres sont exploitées directement par l'entreprise mère. Une septième découverte a subi avec succès les essais de laboratoire, et l'on s'apprêterait à l'exploiter industriellement.

Malgré la difficulté de faire aboutir les processus d'innovation, plusieurs entreprises industrielles publiques et privées du domaine de la sidérurgie, de la chimie, de l'électronique, du machinisme agricole, de l'industrie pharmaceutique et des 
hydrocarbures ont mis en place à leur niveau des CRD (centres de recherchedéveloppement). Globalement, les activités de recherche-développement sont localisées au sein de quinze départements ministériels. Elles sont prises en charge par 126 structures de recherche (centres et unités de recherche ou de recherche-développement, stations d'essais ou d'expérimentation). Elles sont gérées sous quatre types de statuts différents : Etablissement public économique (EPE), Etablissement public administratif (EPA), Etablissement public industriel et commercial (EPIC), Centres et unités de recherche. Quelques 2230 projets de recherche-développement sont actuellement en cours de réalisation, 30 \% d'entre eux sont domiciliés dans le secteur économique (industrie, pharmacie, agriculture...), et les autres associent, la plupart du temps, d'une manière ou d'une autre, une entreprise.

Tableau 2. Inventions déposées par la DRA/SIDER à I'IN A PI

\begin{tabular}{|c|c|c|}
\hline Intitulé & D ate de dépôt & Niveau d'avancement \\
\hline $\begin{array}{l}\text { 1- Poudre delingotièreutiliséeen coulée } \\
\text { continue }\end{array}$ & $\begin{array}{l}\text { 18/ 05/ 87à } \\
10 \mathrm{~h} 50\end{array}$ & $\begin{array}{l}\text { Exploitation industriele } \\
\text { par unefilialedeSDER } \\
\text { (laCODESD) }\end{array}$ \\
\hline $\begin{array}{l}\text { 2- Fabrication d'un fondant sidérurgiqueen } \\
\text { remplacement du spathfluor }\end{array}$ & $\begin{array}{l}\text { 0/10/ 90à } \\
10 \text { h } 10\end{array}$ & Essai delaboratoire \\
\hline $\begin{array}{l}\text { 3- Poudredelubrification delingotièrepour } \\
\text { couléecontinueexceptéedefluor }\end{array}$ & $\begin{array}{c}\text { O2/ 10 90à } \\
10 \text { h } 10\end{array}$ & Essai delaboratoire \\
\hline $\begin{array}{l}\text { 4. Valorisation deproduitsetsousproduits } \\
\text { industries danslafabrication depoudre } \\
\text { d'isolation thermiquepour pochesd'ader }\end{array}$ & $\begin{array}{l}\text { O2/ 10 90à } \\
\text { 10h } 10\end{array}$ & Essai delaboratoire \\
\hline $\begin{array}{l}\text { 5- Procédés defabrication desbriques } \\
\text { réfractairesargileuses denses }\end{array}$ & $\begin{array}{l}\text { 15/ 10 90à } \\
\text { 10h }\end{array}$ & $\begin{array}{l}\text { Exploitation industriele } \\
\text { parlafilialeSDER } \\
\text { (REFRACTAL) }\end{array}$ \\
\hline $\begin{array}{l}\text { 6- Têtedesoudeuseou tordhepour machine } \\
\text { à souder automatiquesousgaz }\end{array}$ & $\begin{array}{l}\text { 28/ 01/ 90à } \\
10 h\end{array}$ & $\begin{array}{l}\text { Exploitation industriele } \\
\text { par SDER }\end{array}$ \\
\hline $\begin{array}{l}\text { 7- Procédédefabrication debriquesà basede } \\
\text { laitier granulédehaut foumeau }\end{array}$ & $\begin{array}{l}\text { 02/ 10 90à } \\
\text { 10h } 25\end{array}$ & $\begin{array}{l}\text { Exploitation industrielle } \\
\text { par odtoi d'unelicenceà } \\
\text { l'entreprisecommunale } \\
\text { ETCA }\end{array}$ \\
\hline $\begin{array}{l}\text { 8- Utilisation du laitier granulédansla } \\
\text { fabrication decarreaux deplandher }\end{array}$ & $\begin{array}{l}\text { 02/ 10/ 90à } \\
10 \mathrm{H} 10\end{array}$ & Essai delaboratoire \\
\hline $\begin{array}{l}\text { 9- Fabrication d'un pigment d'oxydedefer } \\
\text { rouge }\end{array}$ & $\begin{array}{l}\text { 02/ 05/ 90à } \\
14 \mathrm{~h}\end{array}$ & $\begin{array}{l}\text { Essai delaboratoire, } \\
\text { disaussion pour } \\
\text { exploitation industriele }\end{array}$ \\
\hline $\begin{array}{l}\text { 10 Systèmedemesuredeniveau debain } \\
\text { d'acier liquideen lingotièredecoulée } \\
\text { continue-billetes }\end{array}$ & $\begin{array}{l}\text { 06 10 91à } \\
10 \mathrm{~h}\end{array}$ & Essai in situ \\
\hline $\begin{array}{l}\text { 17- Station compactedetraitements deseaux } \\
\text { brutes desurfaceou souterrainespour } \\
\text { l'obtention d'eau potable }\end{array}$ & $\begin{array}{l}\text { O2/ O2/ 92à } \\
\text { 10h }\end{array}$ & $\begin{array}{l}\text { Réalisation de } \\
\text { l'installation àSDER \&t } \\
\text { essai defiabilité }\end{array}$ \\
\hline $\begin{array}{l}\text { 12- Station compactedetraitements deseaux } \\
\text { urbainespar les bactéries }\end{array}$ & 1993 & $\begin{array}{l}\text { Exploitation industriele } \\
\text { par unefilialeSDER } \\
\text { (ENSD) }\end{array}$ \\
\hline $\begin{array}{l}\text { 13- Procédédefabrication decarreaux de } \\
\text { fäencepour revêtementmu ral, à basedu } \\
\text { laitier granulédu haut foumeau d'El Hadjar }\end{array}$ & 1996 & \\
\hline
\end{tabular}

Source: A hmed Slaimi, Dynamique de l'innovation et de l'optimisation des coûts de process dans le secteur industriel, communication aux $3^{\circ}$ Journées d'études sur l'innovation, ADPST, 30-31 mai 2000, p. 15. 
Cet élan est toutefois fortement perturbé par l'instabilité qui a affecté les sociétés publiques et l'organe national de gestion de la recherche. A peine remises des grandes restructurations de 1984, les entreprises ont dû faire face à la politique de démantèlement du secteur public, en cours depuis plus de dix ans. Les organes dirigeants de la recherche ne cessent de changer d'organigramme et de tutelle, répondant à des enjeux politiciens.

\section{CONCLUSION}

Les résultats provisoires de notre recherche font ressortir que l'on est en passe de voir se substituer à une filière politiquement déterminée, à des cursus spécialisés et à des activités professionnelles se limitant au fonctionnement des équipements, une filière économiquement déterminée, avec un contenu polyvalent et une activité professionnelle tournée vers l'innovation. De nouvelles figures professionnelles semblent émerger d'un monde ancien à bout de souffle. Elles sont le résultat de dynamiques locales et se caractérisent donc par des velléités d'autonomie professionnelle peu compatible avec le système politique en vigueur.

Nous avons tenté aussi de rendre compte des bouleversements que l'entreprise a introduit dans les fonctions du chercheur et de la recherche et dans la constitution d'une communauté scientifique à l'échelle nationale et internationale. II semble clair que l'avenir de la recherche et des chercheurs sera encore plus lié au destin de l'entreprise. A vec l'amenuisement du rôle prépondérant de l'Etat dans la vie économique, il se pourrait que seules les recherches s'inscrivant dans les priorités définies par le secteur privé puissent trouver les moyens de leur réalisation.

\section{N OTES}

1 Subventions qui venaient souvent compenser les investissements sociaux que les entreprises publiques consentaient (médecine sociale et du travail, construction de logement, formation professionnelle, construction de route, aides aux autorités locales...), et qui ne sont pas prises en charge dans les économies «libérales ».

2 D'omni présent qu'il était jusqu'à la fin des années 1980, I'Etat est passé, presquesans transition, vers unesituation de retrait économiquetotal, abandonnant mêmela fonction de régulation qu'il assume dans les pays les plus libéraux.

3 KHELFAOUI Hocine, 1999, «Veille technologique et nouveaux modes d'acquisition des savoirs : où en est l'entreprise algérienne? » in N ouveaux modes d'acquisition des savoirs et travail humain, sous la direction de Yassine ESSID, CERETIM, Sfax, pp. 81-94.

4 Voir la synthèse de l'évolution des notions de qualification et de compétence dans : DUBAR Claude, (1996), «La sociologie du travail face à la qualification et à la compétence », Revue de sociologie du travail, $n^{\circ} 2 / 96$, pp. 179-193. 
${ }^{5}$ Cette période s'est certes caractérisée par des liens institutionnels et juridiques intenses, mais elle n'a guère produit d'articulations professionnelles et sociales entre les deux partenaires. Voir à ce sujet notre article «La connexion formation-industrie : les limites de la relation institutionnelle », 1997, Les Cahiers du CREAD , n 41-3, Alger, CREAD, pp. 69-88.

6 On a vu les commentaires lassés de la presse qualifiant ces rencontres de «sempiternelles discussions».

7 En Algérie, toujours qualifiée par ses dirigeants de «pays des miracles », des générations entières ont été et sont encore nourries de discours. Pour perpétuer un discours (il peut arriver, au bout de vingt à trente années, qu'il ne soit plus porteur), il convient seulement d'en changer les termes : c'est suffisant pour le faire passer pour un nouveau discours capable de nourrir une nouvelle génération, etc.

${ }^{8}$ Les entreprises publiques et privées sont désormais sur un pied d'égalité. Elles doivent faire face à la concurrence, mais aussi, et surtout, aux avantages douaniers et autres accordés au bénéfice exclusif des importateurs de produits finis.

9 Elle apparaît par exemple dans les statuts de l'Organisme national de la recherche scientifique (ONRS), premier organisme de gestion de la recherche en Algérie.

10 L'A lgérie est un des pays qui profitele moins dela coopération scientifiqueinternationale, comparé à des pays comme le Maroc ou la Tunisie.

11 Si la recherche scientifique est, comme le montre la sociologie des sciences, le produit de réseaux de plus en plus large, en revanche l'innovation technique est, comme le montrent les sciences de gestion, le fait de fractions de plus en plus larges des employés de l'entreprise.

12 L'Etat commence ces derniers mois à s'intéresser denouveau à la recherche par la promulgation de lois et l'élaboration de budgets. Mais on ne sait pas encore s'il s'agit d'un artifice politique destiné à désamorcer les luttes incessantes des chercheurs et des universitaires pour de meilleures conditions de vieet de travail ou d'une véritable stratégie de promotion des activités scientifiques.

13 Ministère del'Industrie et dela Restructuration, Rôle et missions des universités : renforcement de leurs relations avec la recherche-développement et les entreprises industrielles, 1998.

\section{BIBLIOGRAPHIE}

ALSENE Eric, 1990, «Les impacts de la technologie sur l'organisation », Sociologie du travail, vol. 32, 1990, n³, p. 321-338.

DUBAR Claude, 1996, « La sociologie du travail face à la qualification et à la compétence », R evue de sociologie du travail , n²-96, pp. 179-193.

KHELFAOUI Hocine, 1997, La connexion formation-industrie : les limites de la relation institutionnelle », Les Cahiers du CREA D, Alger, n 41-3, pp. 69-88.

KHELFA OUI Hocine, 1999, «Veille technologique et nouveaux modes d'acquisition des savoirs : où en est l'entreprise algérienne ? » in Nouveaux modes d'acquisition des savoirs et travail humain, sous la direction de Yassine Essid, CERETIM, Sfax, pp. 81-94.

Ministère de l'Industrie et de la Restructuration, 1998, Rôle et missions des universités : renforcement de leurs relations avec la recherche-développement et les entreprises industrielles, document ronéotypé, 58 pages.

RA VAILLE N. et VINCK D., 1999, « Des cultures de conception contrastées », in VINCK D., (ed), Ingénieurs au quotidien, Grenoble, PUG, 1999, pp. 91-111.

SLAIMI A hmed, 2000, «Dynamique de l'innovation et del'optimisation des coûts de process dans le secteur industriel », communication présentée aux $3^{e}$ Journées d'études sur l'innovation, ADPST, Boumerdès, 30/ 31 mai 2000. 



\title{
La G rande Hydraulique dans le Haouz de Marrakech : fascination technologique et émergence du pouvoir des ingénieurs
}

\author{
M ohamed EL FAIIZZ
}

L'eau a eu dans le passé, et continue à avoir de nos jours, une importance telle que son histoire offre une grille de lecture nous permettant de comprendre les processus qui commandent la naissance et le devenir des sociétés locales. Dans le cas du Haouz de M arrakech, le lien entre la question hydraulique et I'histoire se vérifie beaucoup plus que dans les autres régions du Maroc. A partir, d'une part, de l'ancienneté de l'irrigation et d'autre part, de la coexistence dans un même espace de deux, voire trois générations d'appareillages hydrauliques, on a cherchéà suivrel'évolution du projet d'aménagement hydro-agricole. Retracer cette histoire des techniques, dans la longue durée, suppose de s'intéresser au rôle des ingénieurs.

De fait, le projet d'aménagement hydro-agricole du Haouz a une histoire qu'il est possible de reconstituer à travers les rapports et les écrits des ingénieurs depuis le début des années 1960. Mais, dans l'ensemble, on estime que le pas décisif en matière d'aménagement n'a été franchi que durant les cinq premières années de la décennie 1980, où la succession des sécheresses devait hâter le processus de mise en place et de construction des « grands appareillages hydrauliques » de la région ${ }^{1}$.

Les limites de la région du Haouz de Marrakech ont varié au cours des siècles, selon les documents et les auteurs. Sa surface a oscillé entre 350000 ha et 900000 ha rien qu'entre 1961 et 1966. Le sociologue Paul Pascon a retenu pour son objet d'étude un espace plus restrictif, excluant le bassin de la Tessaout et se limitant à la zone comprise entre I'A tlas au sud, les Jbilet au nord, l'oued N'Fis à l'Ouest et la zone d'épandage de I'oued R'Dat à l'Est ${ }^{2}$. Cette restriction se justifiait, il y a vingt ans, quand le projet de transfert des eaux de l'Est était encore à l'étape des études préliminaires. Mais, aujourd'hui, l'extension des équipements hydrauliques (canal de la Rocade, canal T2) 


\section{Carte : périmètre d'irrigation du Haouz}

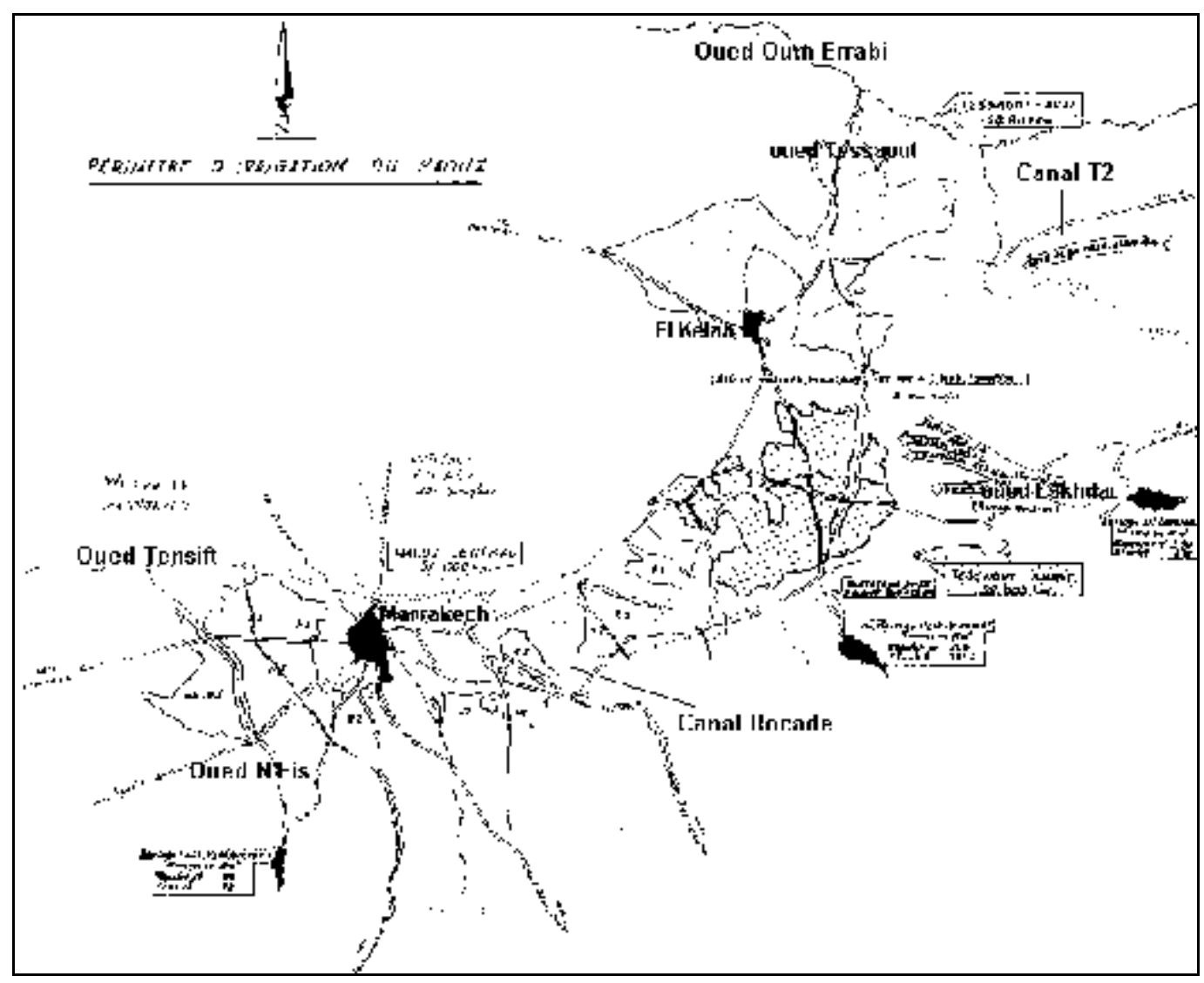

Source: P. PASCON, Le H aouz de M arrakech, t. 1, Rabat, 1983. 
nous oblige à retenir comme définition un Grand Haouz qui couvrirait une partie du bassin de l'Oum er-Rbia et s'étendrait sur la plaine de la Bahira. D'ailleurs, cet élargissement fut consacré tardivement par le décret royal du 21 novembre 1985 qui a étendu la zone d'action de l'Office régional de mise en valeur agricole du Haouz (ORMVAH) aux provinces de M arrakech et d'El Kelâa des Sraghna 3 . Du point devue des conditions de la géographie et du climat, le Haouz appartient à un écosystème aride et semi-aride. Ce sont surtout les réserves en eau du Haut-A tlas qui ont permis, au cours des siècles, des politiques d'aménagement hydro-agricole plus ou moins audacieuses, visant à contrebalancer la rigueur du climat et à accroître les disponibilités alimentaires.

\section{De L'ÉmERgence du POUVOIR Des INGÉNIEURS COLONIAUX AU tRIOMPHE DE L'OPTION TECHNOCRATIQUE}

Beaucoup d'aménageurs pensent que le transfert des eaux d'une région à une autre a été un projet exclusivement colonial. Un premier travail de recherche nous a montré que ce projet est antérieur et participe de la culture hydraulique du « Grand Haouz ». Les « ingénieurs » de la dynastie al mohade au XII ${ }^{\mathrm{e}}$ siècle avaient réussi à transporter l'eau le plus loin possible, là où pouvaient le permettre les conditions financières et technologiques de l'époque médiévale et le génie de leur culture4 ${ }^{4}$. En revanche, l'idée de transférer les eaux de l'Est (oued Lakhdar) vers le Haouz central et occidental remonte bien à l'époque coloniale. Si I'on en croit Paul Pascon 5', cette idée se trouve exprimée pour la première fois dans l'article de M. Martin sur « la question hydraulique dans la région du Haouz », publié dans la Revue de géographie du M aroc, le 4 avril 1927. En réalité, cet ingénieur du corps des Ponts et Chaussées ne parle pas encore explicitement du futur canal de la Rocade, mais il recommande de tirer profit, à la fois, des affluents hydrologiques du bassin de Tensift et du bassin de l'Oum Er-Rabia (Oued Lakhdar et Tessaout). Ce faisant, il oriente les responsables de la politique hydraulique coloniale sur la possibilité de tirer profit des eaux de l'Est, afin d'accélérer la mise en valeur agricole de la région du Haouz.

Ce projet devait servir de prétexte au géographe Jean Brunhes pour attaquer le «lobby » qui fut à l'origine du triomphe de la Grande Hydraulique au Maghreb au tournant des années 1920. La controverse qui va opposer jean Brunnes et M. Martin dépasse donc le cadre étroit du Haouz. Elle suscite un véritable débat entre sciences humaines et sciences dures. 
Jean-Jacques Pérennès situe dans les années 1920 l'option pour les barrages réservoirs et la Grande Hydraulique ${ }^{6}$. II a montré à quel point cette question partageait les spécialistes et opposait surtout les ingénieurs des travaux publics, favorables aux grands appareillages, et les géographes, plus sensibles aux implications d'un tel choix sur le milieu naturel et humain. II revient à Jean Brunhes d'avoir ouvert le débat au-delà des cercles technicistes en le portant sur la place publique. Les travaux de cet érudit ont réussi à retarder, en Algérie, pendant deux décennies la relance del'action en faveur des grands barrages.

La rencontre entre Jean Brunhes et M. Martin a eu lieu en 1928, lors du congrès de I'Eau qui se tint en Algérie au moment de la célébration du centenaire de la colonisation de ce pays. L'ingénieur M. Martin y présente un rapport sur « la technique de construction des grands barrages » dans lequel il considère que pour une mise en valeur intégrale des terres disponibles, il convient $d^{\prime}$ « empêcher la totalité des eaux de ruissellement d'aller à la mer avant d'avoir servi à l'irrigation » ${ }^{7}$. Ce programmen'est pas sans rappeler une formule célèbre et plus ancienne prononcée par Bonaparte devant le Nil : «Pas une seule goutte d'eau à la mer ! ». On comprend, que le géographe Jean Brunhes critique ce «rêve de l'ingénieur Martin ». II reproche, en fait, à tout un corps d'ingénieurs français d'être hanté par la volonté de réaliser une régularisation intégrale des cours d'eau. Jean Brunhes se demande s'il y a intérêt «à tendre les muscles d'un homme jusqu'à la limite extrême de ses forces » :

\begin{abstract}
«La nature, dit-il, doit être traitée comme une chose vivante. Ne soyons pas excessifs vis-àvis d'elle. D'abord, parce qu'il faut être vis-à-vis d'elle- sol ou climat - d'une véritable et sage bonté ; et puis aussi, si cette bonté par hasard vous semblait paradoxale, parce que la nature sait, lorsqu'on lui demande trop, se révolter et prendre sa revanche... O bserve là, ici même, en Algérie et en Espagne; vous lui imposez une camisole de force sous la forme d'un barrage réservoir. "A $h$ ! vous répond-elle, c'est parfait ; mais je me rendrai maîtresse de votre tyrannie." Elle envase, ou elle emporteles pauvres maçonneries humaines! (... ) N e renonçons point à tout projet de barrage réservoir, conclut-il, mais ne mettons pas sans mesure tous nos espoirs en une telle formule périlleuse. »
\end{abstract}

C'est finalement cette « formule périlleuse» qui sera retenue dans les colonies. La thèse de René Arrus a montré que le choix de la Grande Hydraulique s'est effectué non seulement sous la pression des ingénieurs, mais aussi avec l'appui des entreprises métropolitaines des travaux publics et des groupes financiers et bancaires qui les soutiennent. Ce « lobby » trouvait dans les chantiers ouverts des colonies un vaste marché pour la vente du matériel et une zone d'expérimentation pour de nouvelles techniques?. D'autres facteurs ont favorisé I'option pour la Grande Hydraulique au Maroc : l'apparition de surplus céréaliers sur le marché métropolitain et la recherche de cultures 
plus rentables ; la nomination du résident général Steeg plus favorable aux barrages et dont l'ingénieur Martin fut longtemps le disciple ${ }^{10}$; I'envoi de plusieurs missions en Californie, dont la mission Martin, pour s'inspirer du modèle américain. Tous ces éléments vont jouer en faveur de l'adoption de l'agrumiculture et du maraîchage primeur comme cultures valorisant le mieux l'eau des barrages projetés. Le «mythe californien » fonctionnant à plein, le transfert des eaux de l'Est paraissait aux ingénieurs et aux milieux professionnels du début des années 1930 comme une entreprise réalisable à moyen-terme.

Dans un rapport rédigé le 9 décembre 1938, à Marrakech, l'ingénieur des Ponts et Chaussées, Naissant propose une solution à la pénurie d'eau estivale de la ville : il s'agit d'attribuer « un débit prélevé sur l'amenée de l'oued Lakhdar quand elle sera réalisée (8 à 10 ans) ${ }^{11}$. On peut relever que l'amenée (ou canalisation) en question n'est pas encore désignée, dans cette note, sous le nom de « canal de la Rocade », et que la mise à eau est prévue au plus tard pour l'année 1948. La Seconde Guerre mondiale est venue contrarier les prévisions de l'ingénieur. II faut attendre la fin du conflit pour quel'augmentation des ressources hydrauliques devienne à nouveau un objectif prioritaire de la colonisation agricole. C'est alors que se multiplient les pressions pour engager l'action de I'administration sur l'oued Lakhdar. Le transfert des eaux de l'Est est présenté comme pouvant doubler la dotation en eau des terres des colons (de 4000 à $8000 \mathrm{~m} 3 / \mathrm{ha} / \mathrm{an}$ ), et tripler le produit brut de l'arboriculture. Dès 1949, avant même la réalisation des études d'avant-projet du barrage, on met en chantier l'ouvrage detransfert, appelé « canal de la Rocade ». Les travaux commencent par la partie avale et permettent le terrassement de quelques $80 \mathrm{~km}$. Mais le détournement des investissements vers la région de Tadla (Beni Mellal), la montée des luttes nationalistes, et la crise économique et financière du début de l'indépendance, sont autant de raisons qui déterminent l'arrêt des travaux et leur report à une date ultérieure.

Au moment du Protectorat, les discussions furent souvent vives entre ingénieurs du Génie rural, ingénieurs des Travaux publics, administrateurs et colons. La voix des sciences humaines s'est fait entendre en son temps pour montrer les limites d'une option qui fonde tous les espoirs de la population sur la «formule périlleuse » de la Grande Hydraulique. Durant la première décennie de l'indépendance, la controverse fut encore plus animée entre les partisans et les opposants au transfert des eaux. 


\section{ASCENSION ET DÉCLIN DES INGÉNIEURS À «SENSIBILITÉ SOCIALE 》 (1956-1966)}

Le Maroc indépendant a hérite du Protectorat (1912-1956) un engagement en faveur des barrages réservoirs, l'orientation du système de production vers les cultures d'exportation (agrumes, maraîchage primeur) et le rôle prédominant des ingénieurs dans les choix technologiques. La question de l'aménagement du Grand Haouz est remise en débat dès 1958 dans le cadre de la commission spécialisée appelée « Groupe de I'Hydraulique » qui participeà la préparation du plan quinquennal 1960-1964. Cette sorte de brain trust regroupe en majorité des ingénieurs, mais aussi quelques intervenants en sciences humaines et sociales. Les réflexions du groupe débouchent sur la riche expérience del'Office national del'irrigation (ONI) (1960-1966). Nous en rappelons ici les principales réalisations afin de comprendre les choix et les options retenues pour I'aménagement du Haouz ${ }^{12}$.

Créé en septembre 1960, I'ONI a en charge d'administrer cinq périmètres hérités du Protectorat, dont le Haouz. S'ouvre alors une intense période d'études, de projets et d'actions. Le programme d'investigation touche différents domaines : les potentialités hydrauliques des régions, les cultures industrielles, les méthodes culturales, les réformes destructures, etc. Cequi distinguel'ONI d'un bureau d'études ordinaire, c'est sa capacité d'envisager les aménagements hydro-agricoles en liaison avec le changement social dans les campagnes. Les aménageurs de cette époque ne s'occupent pas que de barrages, de tuyauteries et de bornes. Ils cherchent à promouvoir des projets qui économiseraient des devises et qui limiteraient la dépendance économique et financière (choix du gravitaire $)^{13}$; ils veulent engager des actions en faveur des populations rurales les plus défavorisées (petits paysans et paysans sans terre) et des réformes destinées à limiter les effets négatifs de la propriété «latifundiaire».

Néanmoins, le groupe de réflexion de I'ONI ne constitue pas une structure homogène. On y trouve des ingénieurs marocains, des coopérants techniques, des administrateurs et des techniciens formés à différentes écoles (Ponts et Chaussées et Génie rural principalement). C'est cette diversité qui explique la vivacité du débat entre les partisans du transfert des eaux de l'Est vers le Haouz central et les adeptes d'un aménagement prioritaire du périmètre de la Tessaout-amont.

A l'appui de la première thèse, plusieurs études sur les potentialités hydrologiques et pédologiques de la région du Grand Haouz ont révélél'existence d'une discordance entre l'est et l'ouest de la région. L'Est est caractérisé par des ressources hydrauliques abondantes et des terres médiocres. L'Ouest, en revanche, est doté de terres 
de meilleure qualité, mais connaissant d'importants déficits hydriques. D'où l'intérêt d'effectuer le transfert vers le Haouz central où les terres peuvent mieux valoriser l'eau d'irrigation. A cet argument «naturel » viennent s'ajouter d'autres considérations : la concentration des meilleures fermes dans la région de Marrakech ; l'envasement du barrage Lalla Takerkoust sur le N 'Fis (passant de 52 millions m³ en 1935 à 40 millions de m3en 1960 ) ; la surexploitation de la nappe phréatique dont la profondeur est passée de 15 m en 1945 à 40 m en 1960 ; le rôle de Marrakech en tant que pôle de développement régional ; enfin, la nécessité de rentabiliser un investissement existant depuis plusieurs années représenté par lecanal de la Rocade ${ }^{14}$.

Les opposants au projet du transfert avancent, quant à eux, trois principaux arguments: en premier lieu, l'aménagement de l'ensemble du Grand Haouz suppose la réalisation d'études préalables qui peuvent demander beaucoup de temps. Par conséquent, les aménageurs doivent restreindre leur ambition à un projet plus modeste visant l'équipement du périmètre de la Tessaout-amont. En deuxième lieu, l'oued Lakhdar contribue déjà à l'irrigation traditionnelle de quelques 50000 ha dans la plaine des Sraghna. Sa régularisation nécessite non seulement d'imaginer des mécanismes de compensation pour les ayants droit, mais aussi la réal isation d'un plan d'aménagement à l'échelle du bassin de l'Oum ar-Rbia. Enfin, le transfert de l'eau sur une centaine de kilomètres risquerait de susciter des conflits avec les communautés rurales limitrophes, constituées d'irriguants traditionnels se trouvant la plupart du temps frappés par des pénurie d'eau.

Au-delà de l'opposition entre techniciens, le débat recouvre des divergences d'intérêts. Paul Pascon voit, dans les partisans du transfert, les porte-parole des propriétaires fonciers établis dans la banlieue de Marrakech ${ }^{15}$. Mais rien n'empêche de considérer que les opposants au transfert peuvent aussi être les représentants de la fraction d'ingénieurs la plus ouverte au changement social et la plus favorable aux intérêts des couches les plus pauvres de la paysannerie.

Les intérêts locaux des colons en voie d'expropriation ou ceux des grands propriétaires marocains dont les biens ont été séquestrés ne semblent pas avoir joué un rôle important dans le choix des options d'aménagement : « C'est un moment historique en fait, écrit Paul Pascon, où les puissants intérêts fonciers du centre du Haouz se taisent et se terrent, la parole semblant aux seuls techniciens et financiers qui raisonnent sur l'aménagement régional au niveau national ${ }^{16}$ ». 
Il faut l'arbitrage du roi, en visite à Marrakech en janvier 1962, pour que soit finalement décidé de donner la priorité à l'équipement de la Tessaout-amont et à la poursuite des études relatives au transfert des eaux de l'Est vers le Haouz : «II était donc dit, ajoute Paul Pascon, que le premier barrage du Maroc indépendant dans la région du Haouz serait au profit de la petite et moyenne paysannerie. La plupart des terres sont de statut collectif et le melk marocain ne comporte que quelques propriétés de plus de 50 ha ${ }^{17}$. Le projet de transfert est donc à nouveau reporté, non pour des raisons financières, mais parce que les arguments de l'époque coloniale n'étaient plus val ables. La dissolution de I'ONI et son remplacement par des Offices régionaux de mise en valeur agricole (ORMVA) a sinon écarté, du moins marginalisé, la fraction des ingénieurs et des professionnels des sciences humaines et sociales la plus ouverte au « ménagement » $^{18} \mathrm{du}$ territoire agricole qu'à l'aménagement. La voie technocratique est désormais ouverte.

Pour suivre l'évolution de l'idée d'aménagement hydro-agricole du Haouz, il faut maintenant se tourner vers la masse de documents produits par les bureaux d'études et les consultants étrangers.

\section{L'AFFIRMATION DU POUVOIR DE LA «TECHNOSTRUCTURE 》 DES BUREAUX D'ÉTUdES ET DES MINISTĖRES CONCERNÉS PAR L'AMÉNAGEMENT HYDRO-AGRICOLE}

Formulée depuis 70 ans, l'idée d'aménager le Grand Haouz reste une question largement controversée jusqu'à la fin de la décennie 1970. Mais le débat entre les « sciences humaines » et les « sciences dures » prend fin quand les grandes orientations en matière d'aménagement hydro-agricole deviennent du ressort exclusif des bureaux d'études, des ministère des Travaux publics et de l'agriculture et des Offices régionaux de mise en valeur agricole (ORMVA). Certes, la veine critique n'est pas totalement épuisée. Mais elle n'est plus que l'expression de milieux universitaires et académiques qui n'ont pas d'influence sur les décisions des nouveaux aménageurs.

L'analyse du «Plan directeur d'aménagement du Haouz central et de la Tessaoutaval » élaboré par le bureau d'études hollandais Grontmij en 1976 peut permettre de saisir le développement de l'idée d'aménagement du Grand Haouz. Ce document de plusieurs centaines de pages offre un double intérêt : il envisage l'équipement hydroagricole sur le long terme, sur 20 ans et propose des options d'aménagement qui ont fait l'objet d'une concertation étroite avec des responsables placés à différents échelons de l'administration centrale et régionale de l'agriculture et de l'hydraulique. Sans entrer dans les détails techniques, économiques et financiers du projet, on tentera de dégager la 
vision d'ensemble d'un aménagement conçu à l'échelle des deux grands bassins hydrographiques : Le Tensift et l'Oum er-Rbia. Si l'origine de l'idée de « compensation » est encore obscure, elle apparait plus clairement dans le «Plan directeur ». Les aménageurs affirment même que l'année de la mise en eau du canal de la Rocade (ouvrage de transfert et de transport de l'eau) doit coïncider avec l'entrée en fonction du canal T2 pour ne pas léser les irriguants de la plaine des Sraghna. Une autre décision importante est prise en avril 1976: il s'agit d'alimenter la ville de Marrakech à partir de l'eau du canal (40 millions de $\mathrm{m}^{3}$ ), alors qu'une solution précédente envisageait la réalisation d'une conduite prise sur un barrage plus proche $(A \text { it } A d e l)^{19}$.

Le « Plan directeur » retient donc 57 secteurs hydrauliques et 117410 ha à irriguer. Sur la plus grande partie de ces espaces à aménager, les responsables optent pour la formule de l'irrigation par aspersion. Ce choix nous paraît aujourd'hui étonnant. Car il intervient dans une région connue historiquement par la performance de son système gravitaire, sans souci d'économiser des devises rares et sans considérer les coûts supplémentaires en énergie qu'occasionne l'édification des installations de pompage. En ce qui concerne le choix du système de régulation des débits des canaux projetés, les rédacteurs du Plan recommandent d'étudier «les possibilités d'application au Maroc du système de régulation dynamique par télétransmission $\gg 20$.

Un examen des aspects techniques montre que les risques d'envasement ont été prévus, mais quelque peu sous-estimés. «ll faut compter, écrit-on, sur une certaine turbidité de l'eau d'irrigation qui peut poser un problème d'envasement au niveau de la tête morte du canal de la Rocade ». Les ingénieurs hydrauliciens pensent résoudre ce problème en optant pour la construction d'un barrage de compensation du type poids à masque avec un système de chasse en période de crue afin d'évacuer les dépôts ${ }^{21}$.

Le vaste programme d'équipement hydro-agricole du Grand Haouz est aujourd'hui quasiment achevé. Reste à se demander comment et à quel prix ? A-t-on travaillé suffisamment, comme nous y invitait Jean Brunhes depuis près d'un siècle, la fondamentale « liaison entre l'œuvre technique et l'organisation socio-économique »? Nous avons essayé de répondre à ces questions dans une étude récente ${ }^{22}$. En partant des projets des aménageurs, nous avons souligné l'importance des contraintes du financement, analysé la signification et la portée des aménagements. Nous avons montré aussi que face à l'incertitude des bilans de la Grande Hydraulique, la situation d'aujourd'hui est conditionnée par la rigueur des ajustements structurels. 
Les bureaux d'études étrangers ont été sollicités au niveau de la conception générale des aménagements hydro-agricoles. La réalisation des barrages et de l'infrastructure hydraulique constitue un enjeu commercial important et reste, de ce fait, le domaine privilégié de quelques firmes multinationales spécial isées dans le bâtiment et les travaux publics. Citons, à titre d'exemple, Campenon-Bernard, Chaufour-Dumez et Fougerolle qui font partie des entreprises françaises les plus réputées en matière de construction des barrages et qui interviennent depuis longtemps au Maghreb. On découvre, sans surprise, que ces mêmes entreprises françaises ou leurs filiales travaillent dans le Haouz de Marrakech, agissant seules ou en collaboration avec d'autres firmes européennes. Certes, la taille des projets hydro-agricoles et leur complexité justifient le recours à des sociétés compétentes et expérimentées. Mais, comme l'a montré Jean-Jacques Pérennès, la notoriétén'est pas le seul critère de sélection. Le financement joue aussi un rôle de plus en plus déterminant. Souvent, le choix de telle ou telle société tient à sa capacité à apporter un financement par le biais des institutions financières de son pays d'origine ${ }^{23}$.

En comptant toutes ces interventions, l'achat de matériel, les honoraires versés aux ingénieurs, le coût des importations de savoir-faire, on comprend pourquoi la Grande Hydraulique représente un enjeu commercial des plus importants. La facture du transfert de technologie risque d'être lourde, si le pays n'arrive pas à valoriser son potentiel national de recherche et d'études et à développer ses capacités internes d'engineering hydraulique et de maîtrise des métiers de l'eau. II est intéressant, à cet égard, de noter la contribution des Sociétés d'aménagement régional (SAR) du Midi de la France. L'expérience marocaine dans le domaine de l'équipement et la mise en valeur hydroagricole est marquée, dans son ensemble, par le transfert du savoir-faire de ce qu'on a appelé «l'école française de l'eau », et plus précisément de l'action de deux grandes sociétés d'aménagement, la Compagnie nationale d'aménagement de la région du BasRhône Languedoc (CNARBRL) ${ }^{24}$ et la Société du canal de Provence (SCP) ${ }^{25}$.

Le bilan de cette coopération n'a pas encore été fait. Et il faudra un jour l'établir, ne serait ce que pour comprendre la nature des échanges entre les ingénieurs hydrauliciens des deux rives de la Méditerranée et organiser au mieux les circuits du transfert technologique. Mais il reste à savoir si ces sociétés ont œuvré dans le sens du « raccourci technologique » qui est le but présumé de toute action de transfert des techniques, ou bien s'il s'agit plutôt de la volonté d'exporter un modèle « clef en main ». Dans ce cas, la région du Haouz serait une zone de plus dans le vaste territoire du «mimétisme technologique $\gg^{26}$. 
Aujourd'hui, le processus des réformes de la Grande Hydraulique, tel que le conçoit la Banque mondiale, paraît engagé. Les Offices marocains ont entamé une phase complexe d'ajustements qui touchent aussi bien leur vision techniciste des problèmes de l'irrigation que leur rôle dans l'agriculture, leurs rapports avec le monde paysan, leur organisation et leur mode de fonctionnement. Sans remettre en cause le bien fondé de ces réformes, il nous semble que là aussi la «greffe des modèles » comporte un gros risque de désenchantement. Car le problème de l'aménagement hydro-agricole du Haouz ne réside pas dans le passage d'une comptabilité publique à une comptabilité privée, ni dans la mise en place des SIG (Systèmes d'information et de gestion) et des instruments de télédétection et de contrôle de l'irrigation. Ce ne sont là que des outils de gestion dont le développement doit être maîtrisé et réal isé en fonction des besoins réels de l'entreprise. L'expérience a d'ailleurs montré que lorsque les conditions méthodologiques et sociologiques de la mise en œuvre de tels outils ne sont pas étudiées, le transfert risque d'échouer, aboutissant, dans le meilleur des cas, à des systèmes peu opérationnels. En tout cas, quel que soit l'angle où l'on se place pour examiner la réforme du modèle de la Grande Hydraulique dans le Haouz, il n'est pas possible de continuer à ignorer la dimension socio-structurelle dans les changements à venir.

A près une longue expérience parmi les ingénieurs hydrauliciens de la Société du canal de Provence, ceux-là même qui furent impliqués dans le Haouz et dans d'autres régions du Tiers Monde, Michel Marié nous donne sa conviction «qu'un dispositif technique n'a de chance d'être rentable et de bien fonctionner que si, paradoxalement, il a su affronter des sociétés locales assez fortes pour lui résister et donc composer avec de l'épaisseur sociale ». L'aménagement ne réussit, en définitive, que lorsqu'il s'accompagne d'une certaine dose de «ménagement du territoire », lequel se définit comme étant « la capacité des institutions de l'aménagement à autoréguler, c'est-à-dire à réévaluer en permanence les termes de leur action en fonction des forces en présence ${ }^{27}$. Dans le cas du Haouz, la société locale fut pratiquement absente au moment des grandes décisions d'aménagement. La place fut longtemps occupée par les techniciens qui intervenaient à tous les échelons de l'administration hydro-agricole, laissant peu d'initiative aux paysans. L'énorme potentiel d'engineering, de savoir-faire et de « savoir-gérer » que recèle l'hydraulique dite traditionnelle fut partiellement détruit et considéré comme condamné. 
Rien n'exprime mieux la volonté d'instaurer une agriculture industrielle, une « agriculture sans paysans », que la disparition des documents des bureaux d'études et des milieux techniques, de toute référence aux besoins réels des fellahs et à leurs aspi rations. Le concept de « participation paysanne», cher à la sociologie de Paul Pascon, a longtemps été occulté. II réapparaît aujourd'hui dans la littérature technique des Offices, après quarante ans d'absence. Les difficultés de recouvrement des charges de l'irrigation ne sont pas étrangères à ce regain d'intérêt pour l'organisation des communautés rurales. Tout le pari de la situation actuelle réside dans la capacité à repenser la machinerie lourde de la Grande Hydraul ique et à lubrifier ses mécanismes en y introduisant de fortes doses de «ménagement ». On a vu que la question de l'eau ne se réduit pas à un simple transfert de techniques. Elle constitue, en tout cas, une affaire trop sérieuse pour rester du domaine exclusif des ingénieurs, des techniciens et des bureaux d'étude.

\section{N OTES} 1976

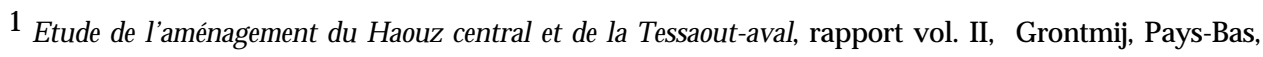

2 PASCON P., LeH aouz de M arrakech, I, Rabat, 1983, p. 24.

${ }^{3}$ La superficie du Grand Haouz qui coïncide avec la zone d'action actuelle del'ORMVAH, s'étend sur une superficie totale de $7000 \mathrm{~km}^{2}$. Les terres agricoles représentent les deux tiers de cette superficie (soit 470000 hectares). Le canal Rocade, achevé en 1985, amène l'eau de l'oued Lakhdar (à l'est) et la transporte sur $120 \mathrm{~km}$ pour arroser le Haouz central et le périmètre du N'Fis, situé à l'ouest de Marrakech. Le canal T2 (Tadla-Tassaout), moins étendu, a pour fonction d'irriguer le périmètre de la ville d'El Kelaa des Sraghna et compenser ainsi les prélèvements effectués par le canal Rocade sur les ressources hydrauliques de cette région.

4 EL FAÏZ M., «Pour une histoire de longue durée des aménagements hydro-agricoles dans le Haouz de Marrakech », in Grands appareillages hydrauliques et sociétés locales en M éditerrané, Michel Marié et A hmed Bencheikh (dir.), Ed. Ecole des ponts et chaussées, Paris, 1994 ; «Les aménagements hydro-agricoles dans le Haouz : histoire d'une idée», in Séminaire international «Cultures techniques de l'eau et régulation à l'échelle régionale », 4-5 novembre 1996 (Avignon, France), publié in Cultures, usages et stratégies de l'eau en M éditerranée occidentale, MARIÉ M. et alii (dir.), Ed. L'Harmattan, Paris, 1999.

5 PASCON P., Le H aouz de M arrakech, II, Rabat, 1983, p. 526.

6 PERENNESJ-J., L'eau et les hommes au M aghreb, Ed. Karthala, Paris, 1993, p. 127.

7 BRUNHES J., «Le problème technique et économique des grands barrages-réservoirs en Algérie, in Centenaire de l'A Igérie, congrès de la Colonisation, Alger, 1930, p. 11.

8 Ibid., p. 13.

${ }^{9}$ ARRUS R., L'eau en A Igérie, Ed. PUG, Grenoble, 1985, p. 154.

$10 \mathrm{~T}$. Steeg est nommé résident général du Maroc en 1926. On l'appela le «gouverneur de l'eau » en Algérie, dans les années vingt. Cf. STEEG T., La paix française en A frique du N or d, Paris, Ed. Félix Alcan, 1926 et plus particulièrement la conférence intitulée «Une politique africaine de l'eau », pp. 121-137. 
${ }^{11}$ Archives de l'ORMVAH, dossier Agdal.

12 EL FAÏZ M. et SEDDIKI A., Essai sur le capitalisme agraire périphérique : cas de l'irrigation au M aroc, thèse de $3^{\mathrm{e}}$ cycle, Grenoble, 1979.

13 L'irrigation gravitaire, la plus ancienne dans le Haouz, consiste à apporter l'eau aux champs en profitant de la pente du terrain. Le système del'aspersion ou del'irrigation local isée utilise, quant à lui, l'eau mise sous pression.

14 PASCON P. et DUCROCQ M., «La miseen valeur du périmètre dela Tessaout », in H ommes, Terres et Faux, 1973, pp. 24-25.

15 Ibid., p. 25.

16 Ibid., p. 25.

17 Etudedel'aménagement et dela miseen valeur du H aouz central et de la Tessaout-aval - Plan directeur, vol. VII, rapport de synthèse, Grontmij, Pays-Bas, décembre 1976, p. 40.

18 MARIÉ M., «Aménager ou ménager le territoire », in A nnales des P onts et Chaussées, $n^{\circ}$ 77, 1996.

${ }^{19}$ Etude de l'aménagement et de la mise en valeur du H aouz central , op. cit., p. 40.

20 lbid., p. 37.

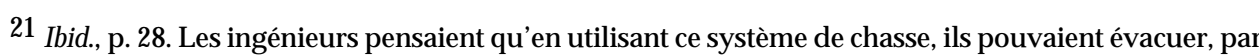
en bas, les dépôts s'accumulant au fond du barrage.

22 EL FAïZ M., «Modèle de la Grande Hydraulique dans le Haouz de Marrakech : bilans d'un aménagement frontal », in Séminaire international «Echelles et modèles de gestion de l'eau », Marrakech, 79 octobre, 1998. Publié in «Les usages de l'eau : échelles et modèles en Méditerranée », René ARRUS et alii (dir.), Economies et Sociétés, Hors-série, Presses de l'ISMEA, Paris, 2000.

23 PERENNESJ-J., op. cit., p. 378.

24 SOUCHON M-F., La Compagnie nationale d'aménagement de la région du Bas-Rhône Languedoc, Ed. Cujas, Paris, 1968 et LAM OUR P., Le Cadran Solaire, Ed. R. Laffont, Paris, 1980.

25 Cf. les travaux de Michel Marié, «Pour une anthropologie des grands ouvrages, le canal de Provence », in Les Annales de la recherche urbaine, XXI, 1984 ; Territoires hydrauliques : la Société du canal de Provence dix ans après, LATTS, ENPC, Paris, 1993.

${ }^{26}$ LAMBERT D-Cl., Le mimétisme technologique des Tiers-M ondes, Ed. Economica, 1983.

27 MARIÉ M., op. cit., pp. 67-68. 
